J. Krieger · W. Schlag

\title{
Non-generic blow-up solutions for the critical focusing NLS in 1-D
}

Received October 26, 2007

Abstract. We consider the $L^{2}$-critical focusing non-linear Schrödinger equation in $1+1$-d. We demonstrate the existence of a large set of initial data close to the ground state soliton resulting in the pseudo-conformal type blow-up behavior. More specifically, we prove a version of a conjecture of Perelman, establishing the existence of a codimension one stable blow-up manifold in the measurable category.

Keywords. Non-linear Schrödinger equations, $L^{2}$-critical NLS, pseudo-conformal blow-up

\section{Introduction}

We consider the critical focusing NLS in 1-d of the form

$$
i \partial_{t} \psi+\partial_{x}^{2} \psi=-|\psi|^{4} \psi, \quad i=\sqrt{-1}, \psi=\psi(t, x),
$$

with $\psi$ complex-valued. It is well-known that this equation permits standing wave solutions of the form

$$
\phi(t, x)=e^{i \alpha t} \phi_{0}(x, \alpha), \quad \alpha>0 .
$$

Indeed, requiring positivity and evenness in $x$ for $\phi_{0}(x, \alpha)$ implies for example

$$
\phi_{0}(x, \alpha)=\frac{\alpha^{1 / 2}(3 / 2)^{1 / 4}}{\cosh ^{1 / 2}(\alpha x / 2)} \text {. }
$$

Another remarkable feature of the equation 1.1 is the large symmetry group carrying solutions into solutions: this is generated by

- Galilei transformations:

$$
\begin{aligned}
& \psi(t, x) \mapsto e^{i\left(\gamma+v x-v^{2} t\right)} e^{-i(2 t v+\mu) p} \psi(t, x)=e^{i\left(\gamma+v x-v^{2} t\right)} \psi(t, x-2 t v-\mu), \\
& p=-i \frac{d}{d x}
\end{aligned}
$$

J. Krieger: Department of Mathematics, Science Center, Harvard University, 1 Oxford Street, Cambridge, MA 02138, USA; e-mail: jkrieger@math.harvard.edu

Department of Mathematics, University of Chicago, 5734 South University Avenue, Chicago, IL 60637, USA; e-mail: schlag@ math.uchicago.edu

Mathematics Subject Classification (2000): Primary 35Q55, 35Q51 
- $\operatorname{SL}(2, \mathbb{R})$-transformations:

$$
\psi(t, x) \mapsto(a+b t)^{-1 / 2} e^{\frac{i b x^{2}}{4(a+b t)}} \psi\left(\frac{c+d t}{a+b t}, \frac{x}{a+b t}\right), \quad\left(\begin{array}{ll}
a & b \\
c & d
\end{array}\right) \in S L(2, \mathbb{R}) .
$$

Observe that the latter subsume rescalings $\psi(t, x) \mapsto a^{1 / 2} \psi\left(a^{2} t, a x\right)$ while the former subsume phase shifts $\psi(t, x) \rightarrow e^{i \gamma} \psi(t, x)$ as well as translations. We usually identify a matrix $\left(\begin{array}{ll}a & b \\ c & d\end{array}\right) \in S L(2, \mathbb{R})$ with the corresponding transformation. It is the $S L(2, \mathbb{R})$ transformations that distinguish the critical NLS from the sub- and supercritical NLS, and allow us to exhibit explicit blow-up solutions: indeed, fixing $\left(\begin{array}{ll}a & b \\ c & d\end{array}\right) \in S L(2, \mathbb{R})$, we have the explicit solution

$$
f(t, x)=(a+b t)^{-1 / 2} e^{i \frac{c+d t}{a+b t}} e^{\frac{i b x^{2}}{4(a+b t)}} \phi_{0}\left(\frac{x}{a+b t}, 1\right),
$$

which blows up for $t=-a / b$. Fixing $a \sim 1, b \sim-1$, it is then a natural question to ask whether one may perturb the initial data of $[1.2)$ at time $t=0$ so that the corresponding solution exhibits the same type of blow-up behavior. More precisely, the solution should asymptotically behave like $\sqrt{\frac{1}{T-t}} e^{i \Psi(t, x)} \phi\left(\frac{x-\mu(t)}{T-t}\right)$ for a bounded function $\mu(t)$ and suitable Schwartz function $\phi$, with blow-up time $T$. The recent work of MerleRaphael [MeRa] has demonstrated that this is generically impossible, i.e., there are open sets of initial data containing $f(0, x)$ in their closure ${ }^{1}$ and such that their blow-up behavior is of the following type, which we henceforth refer to as 'generic':

$$
\psi(t, x) \sim e^{i \mu(t)} \lambda^{1 / 2}(t) \phi(\lambda(t) x), \quad \lambda(t) \sim \sqrt{\frac{\log |\log (T-t)|}{T-t}} .
$$

Blow-up solutions of this type were first constructed in a remarkable paper by G. Perelman [Per], but for non-generic initial data sets. This blow-up rate was shown to be stable in [Ra]. Moreover, in [MeRa] the authors showed that for initial data in a sufficiently small neighborhood of $f(0, x)$ the only possible blow-up speeds are the generic speed or else at least as fast as the explicit speed; we now refer to the latter as 'non-generic'. The issue remains as to whether perturbations of the initial data $f(0, x)$ in certain directions would result in the non-generic blow-up type. The first and to our knowledge only result of this type was established by Bourgain-Wang $[\mathrm{BW}] \mathrm{L}^{2}$ and asserts the following:

Theorem 1.1 (Bourgain-Wang). Let $z_{\phi}$ be the local-in-time solution of

$$
i \psi_{t}+\Delta \psi+|\psi|^{4} \psi=0, \quad \psi(0)=\phi,
$$

which for smooth $\phi$ exists on an interval $[-\delta, \delta]$ for $\delta=\delta(\phi)$ small enough. Then provided $\phi$ is smooth and vanishes sufficiently fast at 0 , i.e. $|\phi(x)| \lesssim|x|^{A}$ for A large

\footnotetext{
1 With respect to any reasonable norm.

2 The authors state this theorem for the case of $d=1,2$ dimensions.
} 
enough ${ }^{3}$ there exists a smooth $w(t, x)$ in a suitable function space with $w(0, x)=0$ and such that

$$
\psi(t, x)=t^{-1 / 2} e^{\frac{x^{2}-4}{4 i t}} \phi_{0}\left(\frac{x}{t}, 1\right)+z_{\phi}(t, x)+w(t, x)
$$

solves (1.1) on $[-\delta, 0]$. One may let $\delta \rightarrow \infty$ by letting $\phi \rightarrow 0$.

The key behind this result is to first undo the blow-up by applying a pseudo-conformal transformation $C^{-1}$ where $C \psi(t, x)=t^{-1 / 2} e^{x^{2} / 4 i t} \psi(x / t,-1 / t)$ and then employ the properties of the linear evolution associated with the linearization around the standing wave $e^{i t} \phi_{0}(x)$. More precisely, one passes to the vector-valued function $\left(\begin{array}{l}\psi(t, x) \\ \bar{\psi}(t, x)\end{array}\right)$ and observes that if $\psi(t, x)=e^{i t}\left(\phi_{0}(x, 1)+u(t, x)\right)$ solves (1.1) then

$$
\left(i \partial_{t}+\mathcal{H}\right)\left(\begin{array}{l}
u(t, x) \\
\bar{u}(t, x)
\end{array}\right)=N(u)
$$

where we put

$$
\mathcal{H}=\left(\begin{array}{cc}
\partial_{x}^{2}-1+3 \phi_{0}^{4}(x, 1) & 2 \phi_{0}^{4}(x, 1) \\
-2 \phi_{0}^{4}(x, 1) & -\partial_{x}^{2}+1-3 \phi_{0}^{4}(x, 1)
\end{array}\right)
$$

and $N(u)$ is of order $\geq 2$ in $u$. The spectral properties of the operator $\mathcal{H}$ are well-known after the pioneering work of Weinstein [Wei1] as well as Buslaev-Perelman [BusPer] and Perelman [Per]. In particular, the linear equation $\left(i \partial_{t}+\mathcal{H}\right)\left(\frac{u}{u}\right)=0$ only displays algebraic instabilities. More precisely, the spectrum of $\mathcal{H}$ has essential part $(-\infty,-1] \cup[1, \infty)$ and discrete spectrum $\{0\}$ of geometric multiplicity 2 and algebraic multiplicity 6 . A solution $\Phi(x)=\left(\begin{array}{c}\phi(x) \\ \bar{\phi}(x)\end{array}\right)$ in the generalized root space satisfies for example $\left\|e^{i t \mathcal{H}} \Phi(x)\right\|_{L_{x}^{2}} \lesssim$ $\left(1+t^{3}\right) \int_{-\infty}^{\infty} e^{-c|x|}|\Phi(x)| d x$. In order to counteract this growth behavior at infinity, Bourgain and Wang use the Ansatz $u(t, x)=C^{-1} z_{\phi} e^{-i t}+\tilde{w}(t, x)$ (see (1.3) ) and then observe that the non-linearity of the resulting equation for $\left(\frac{\tilde{w}}{\tilde{w}}\right)$ decays sufficiently rapidly at infinity (due to the local decay of $C^{-1} z_{\phi} e^{-i t}$ ) that it overwhelms any losses due to the algebraic instability of $\mathcal{H}$. The fact that the 'static coupling' (1.3) barely exploits the symmetries of the equation and in particular does not allow the standing wave to 'drift' certainly implies the suboptimality $\sqrt{4}$ of Theorem 1.1 .

Indeed, a careful analysis of the root space of $\mathcal{H}$ as in [Wei1] shows that five of the generalized root modes (the 'good modes') 'are due to' the internal symmetries of (1.1), while there is one 'exotic mode' (see the ensuing discussion). This intimates that upon applying suitable internal symmetries to the standing wave $e^{i t} \phi_{0}(x, 1)$ in time dependent fashion (i.e., using a modulation-theoretic approach), one should be able to control the root part of the radiation corresponding to the good modes, and indeed obtain a codimension 1 stable manifold of initial data (due to the 'exotic mode' which cannot be so controlled) resulting in the non-generic blow-up profile:

\footnotetext{
3 The numerology in [BW] appears to imply $A \geq 16$.

4 In the sense that the set of initial data resulting in the non-generic blow-up should be significantly larger than indicated there.
} 
Conjecture 1.2 (Galina Perelman). There exists a codimension 1 manifold of initial data resulting in the non-generic blow-up behavior.

We note that this is also implicitly mentioned, although in less precise form, in [B].

This falls in neatly with recent results in [?] and [KriSch1], the latter closely following the former, which in the context of the $L^{2}$-supercritical NLS (the cubic in 3-d in [Sch] and the full supercritical range in 1-d in [KriSch1]) established existence of codimension 1 manifolds of initial data resulting in globally (for $t \rightarrow+\infty$ ) smooth solutions. The codimension 1 here has to do with one exponentially unstable mode (in the forward time direction); the generalized root space has only dimension 4 , in one-one correspondence with the internal symmetries.

However, the critical case differs in a pivotal aspect from the supercritical one: this is due to the nature of the dynamics governing the 'exotic mode'. Recall that this mode, together with one of the 'geometric modes', turn into the exponentially growing and decaying modes corresponding to the purely-imaginary eigenvalues in the supercritical case. The latter are controlled by a hyperbolic ODE. As observed in [Sch] and later [KriSch1], this hyperbolic ODE produces good decay behavior at infinity, provided this is coupled with suitable initial conditions. In particular, the linear theory in [KriSch1] allows one to get by with rather weak local decay estimates for the dispersive part of the radiation, such as $\left|\phi U_{\mathrm{dis}}(t, \cdot)\right| \lesssim\langle t\rangle^{-1-\epsilon}$ for a Schwartz function $\phi$.

In the critical case, the imaginary eigenvalues merge at zero. The evolution of the 'exotic root mode' which is not due to internal symmetries is then controlled by a Riccati type ODE, 3.23. It is then clear that the linear estimates alone derived in [KriSch1] are nowhere near enough to control the evolution of this mode, in a fashion similar to [Sch] or [KriSch1]. Instead, we have to exploit subtle null-structures in the non-linearity, i.e., exploit the oscillatory behavior of the radiation part, to eke out additional decay in time. Such estimates, while similar in nature to null-form estimates for non-linear wave equations and the defocusing NLS, appear completely new in this context, especially since we do not work with the ordinary Fourier transform but with the distorted Fourier transform. In particular, the considerations in Section 5.1, which produce almost optimal local decay for the dispersive radiation part, appear of interest in their own right, and might be useful in other contexts. The crucial observation in this section is that the expression $|U|^{2}$ has a certain smoothing property.

In this paper we prove the following version of Conjecture 1.2 let5

$$
\mathcal{T}_{\infty}=e^{-i\left(v_{\infty}^{2} s+\gamma_{\infty}+v_{\infty} y\right)} e^{i\left(2 v_{\infty} s+y_{\infty}\right) p}\left(\begin{array}{cc}
a_{\infty} & b_{\infty} \\
0 & a_{\infty}^{-1}
\end{array}\right) .
$$

Also, let the generalized root space of $\mathcal{H}$ be generated by the (vector-valued) Schwartz functions $\eta_{i \text {,proper }}=\left(\begin{array}{l}\eta_{i, \text { proper }}^{1} \\ \bar{\eta}_{i, \text { proper }}^{1}\end{array}\right), i=1, \ldots, 6$, and that of $\mathcal{H}^{*}$ be generated by the Schwartz functions $\xi_{i, \operatorname{proper}}(x), i=1, \ldots, 6$ (see. the ensuing discussion).

Our main result is the following

\footnotetext{
5 We identify the matrix with its associated transformation.
} 
Theorem 1.3. Fix real parameters $\lambda \sim 1, \beta \sim 1, \omega \lesssim 1, \gamma \lesssim 1, \mu \lesssim 1$. Given a vector-valued function $x \mapsto\left(\begin{array}{c}U \\ \bar{U}\end{array}\right)(0, x)$ satisfying $\left\langle\left(\begin{array}{c}U \\ \bar{U}\end{array}\right)(0, \cdot), \xi_{i, p r o p e r}\right\rangle=0$ for all $i$, as well as the smallness condition $\left\||U(0, \cdot) \||<\delta\right.$ for a suitable norm $\left.{ }^{6}\right\||\cdot| \|$ and sufficiently small $\delta>0$, there exist numbers $\tilde{\lambda}_{i} \in \mathbb{R}$ with $\left.\left|\tilde{\lambda}_{i}\right| \lesssim\|U(0, \cdot)\|\right|^{2}$ and parameters $\left\{a_{\infty}, b_{\infty}, v_{\infty}, y_{\infty}, \gamma_{\infty}\right\}$ with $\left|a_{\infty}-\lambda\right| \lesssim\left\|\left|U(0, \cdot)\left\|\left.\right|^{2},\left|b_{\infty}-\beta \lambda\right| \lesssim\right\| U(0, \cdot)\right|\right\|^{2}$, $\left|v_{\infty}-\beta \lambda \mu / 2-\omega\right| \lesssim|| U(0, \cdot)||^{2},\left|y_{\infty}-\lambda \mu\right| \lesssim|| U(0, \cdot) \|^{2}, \gamma_{\infty}=\gamma_{\infty}(\gamma, \lambda, \beta, \omega, \mu)+$ $O\left(|\|U(0, x)\||^{2}\right)$, such that the initial data

$$
\psi(0, x):=W(0, x)+\mathcal{T}_{\infty}^{-1}\left[U(0, x)+\sum_{i=1}^{6} \tilde{\lambda}_{i} \eta_{i, \text { proper }}^{1}\right]
$$

lead to solutions of 1.1 blowing up in finite time according to the non-generic profile, where

$$
W(0, x)=e^{i(\gamma+\omega(x-\mu))} e^{-i \frac{\beta}{4} \lambda^{2}(x-\mu)^{2}} \sqrt{\lambda} \phi_{0}(\lambda(x-\mu), 1) .
$$

More precisely, the solution decouples as

$$
\psi(t, x)=e^{i(\gamma(t)+\omega(t)(x-\mu(t)))} e^{-i \frac{\beta(t)}{4} \lambda^{2}(t)(x-\mu(t))^{2}} \sqrt{\lambda(t)} \phi_{0}(\lambda(t)(x-\mu(t)), 1)+R(t, x),
$$

where $\lambda(t) \sim 1 /(T-t)$ for a suitable $T>0$, and $\mu(t)$ is bounded, while we have the bounds

$$
\sup _{0 \leq t<T}\|R(t, x)\|_{L_{t}^{\infty}} \lesssim \delta, \quad\|R(t, x)\|_{H^{1}} \lesssim \delta(T-t)^{-1}, \quad 0 \leq t<T .
$$

In particular, $\|\psi(t, x)\|_{H^{1}} \sim(T-t)^{-1}$ for $0 \leq t<T$.

Remark. We observe that this result would imply Conjecture 1.2 if we could show Lipschitz continuous dependence of the $\tilde{\lambda}_{i}, a_{\infty}$ etc. on $U(0, x)$, along the lines of [KriSch1]. However, we cannot establish this. Indeed, even demonstrating the possibility or impossibility of choosing these parameters in continuous fashion appears extremely difficult.

We now outline the strategy used to prove this theorem; there are the following four stages:

Stage A: Setting up the equations for radiation part and modulation parameters

Instead of the static coupling 1.3 , we make the Ansatz

$$
\begin{aligned}
\psi(t, x) & =W(t, x)+R(t, x), \\
W(t, x) & =e^{i \theta(t, x)} e^{-i \frac{\beta(t)}{4} \lambda^{2}(t)(x-\mu(t))^{2}} \sqrt{\lambda(t)} \phi_{0}(\lambda(t)(x-\mu(t)), 1) .
\end{aligned}
$$

In order to ensure that this solution behaves like a non-generic blow-up solution, we impose the condition $\lambda(t) \sim 1 /\left(t_{*}-t\right)$ for suitable $t_{*} \in \mathbb{R}_{>0}$. We shall similarly have to carefully specify the 'asymptotic behavior' of the remaining parameters as we approach

\footnotetext{
6 See Definition 4.4
} 
blow-up time. In order to specify the evolution of these parameters, we impose suitable orthogonality conditions: letting $\xi_{i \text {,proper }}, i=1, \ldots, 6$, denote a certain basis of the generalized root space of $\mathcal{H}^{*}$ (recall (1.4) ) to be specified below, we impose 7

$$
\begin{aligned}
& \left\langle\left(\begin{array}{l}
R \\
\bar{R}
\end{array}\right), \xi_{i}\right\rangle=0, \\
& \xi_{i}=\left(\begin{array}{cc}
e^{i \Psi(t, x)} & 0 \\
0 & e^{-i \Psi(t, x)}
\end{array}\right) \sqrt{\lambda(t)} \xi_{i, \operatorname{proper}}(\lambda(t)(x-\mu(t))), \quad i=2, \ldots, 6,
\end{aligned}
$$

where we have introduced the notation

$$
\Psi(t, x)=\theta(t, x)-\frac{\beta(t)}{4} \lambda^{2}(t)(x-\mu(t))^{2} .
$$

For later reference, we define $\eta_{i}$ correspondingly, with $\xi_{i \text {,proper }}$ replaced by $\eta_{i \text {,proper }}$. This is analogous to the procedure in [Sch], [KriSch1], where the generalized root space is only 4-dimensional. The above orthogonality condition then implies that at time $t$ the radiation part when projected onto the generalized root space of the instantaneous linearization around the drifting soliton gives zero. Note that the fact the we no longer work with a static standing wave forces us to work with modifications of the operator $\mathcal{H}$.

Instead of working with the formulation (1.5), we then revert to a 'different gauge' as in $\left[\mathrm{BW}\right.$. Specifically, we apply a suitable transformation $\mathcal{T}_{\infty}$,

$$
\mathcal{T}_{\infty}=e^{-i\left(v_{\infty}^{2} s+\gamma_{\infty}+v_{\infty} x\right)} e^{i\left(2 v_{\infty} s+y_{\infty}\right) p}\left(\begin{array}{cc}
a_{\infty} & b_{\infty} \\
0 & a_{\infty}^{-1}
\end{array}\right), \quad p=-i \frac{d}{d x},
$$

to $\psi(t, x)$ which is to undo the singular behavior and should map the blow-up time $t_{*}$ to $s=\infty$. To see how the 'coefficients at infinity' $a_{\infty}$ etc. should be chosen, we observe that

$$
\left(\mathcal{T}_{\infty} F\right)(s, y)=e^{-i \tilde{\Psi}_{\infty}(s, y)} \lambda_{\infty}^{-1 / 2}(s) F\left(\int_{0}^{s} \lambda_{\infty}^{-2}(\sigma) d \sigma, \lambda_{\infty}^{-1}(s) y+\mu_{\infty}(s)\right),
$$

where

$$
\begin{gathered}
\lambda_{\infty}(s)=a_{\infty}+b_{\infty} s, \quad \mu_{\infty}(s)=\frac{2 v_{\infty} s+y_{\infty}}{a_{\infty}+b_{\infty} s} \\
\tilde{\Psi}_{\infty}(s, y)=v_{\infty}^{2} s+\gamma_{\infty}+v_{\infty} y-\frac{b_{\infty}\left(y+2 v_{\infty} s+y_{\infty}\right)^{2}}{4\left(a_{\infty}+b_{\infty} s\right)}
\end{gathered}
$$

and therefore

$$
\begin{aligned}
e^{-i s}\left(\mathcal{T}_{\infty} W\right)(s, y)= & e^{-i\left(s+\tilde{\Psi}_{\infty}(s, y)\right)+i \Psi\left(t(s), \mu_{\infty}(s)+\lambda_{\infty}^{-1}(s) y\right)} \lambda_{\infty}^{-1 / 2}(s) \lambda^{1 / 2}(t(s)) \\
& \cdot \phi_{0}\left(\lambda(t(s))\left(\mu_{\infty}(s)-\mu(t(s))+\lambda_{\infty}^{-1}(s) y\right), 1\right),
\end{aligned}
$$

7 The root functions $\xi_{i \text {,proper, }}, i=1, \ldots, 5$, here are chosen to be the 'good modes' in one-one relation with the internal symmetries, while the root function $\xi_{6}$, proper is the 'exotic mode' due to the degeneracy in the critical case. 
where we have put $t(s)=\int_{0}^{s} \lambda_{\infty}^{-2}(\sigma) d \sigma=a_{\infty}^{-1} s /\left(a_{\infty}+b_{\infty} s\right)$. The above suggests that we should impose $\lambda(t(s)) \sim \lambda_{\infty}(s), \mu_{\infty}(s) \sim \mu(t(s))$ as $s \rightarrow \infty$ in a precise sense to be specified. In particular, we have $t_{*}=1 / a_{\infty} b_{\infty}$ for the blow-up time.

We shall now work with the vector-valued function

$$
\left(\begin{array}{l}
U \\
\bar{U}
\end{array}\right):=\mathcal{M} \mathcal{T}_{\infty}\left(\begin{array}{l}
R \\
\bar{R}
\end{array}\right), \quad \mathcal{M}=\left(\begin{array}{cc}
e^{-i s} & 0 \\
0 & e^{i s}
\end{array}\right)
$$

Then introduce the functions $\tilde{\eta}_{i}=\mathcal{M} \mathcal{T}_{\infty} \eta_{i}, \tilde{\xi}_{i}=\mathcal{M} \mathcal{T}_{\infty} \xi_{i}$. One deduces the following equation for $\left(\begin{array}{c}U \\ \bar{U}\end{array}\right)$ :

$$
\begin{aligned}
i \partial_{s}\left(\begin{array}{c}
U \\
\bar{U}
\end{array}\right)+\mathcal{H}(s)\left(\begin{array}{c}
U \\
\bar{U}
\end{array}\right)= & -i\left(\dot{\lambda} \lambda^{-1}-\beta v^{2}\right)\left(\tilde{\eta}_{2}-\beta \tilde{\eta}_{5} / 2+\omega \tilde{\eta}_{4}\right) \\
& +\frac{i}{4}\left(\dot{\beta}+\beta^{2} v^{2}\right) \tilde{\eta}_{5}+i\left(v^{2}-\dot{\gamma}+v^{2} \omega^{2}\right) \tilde{\eta}_{1}-i\left(\dot{\omega}+\beta \omega v^{2}\right) \tilde{\eta}_{4} \\
& -i v\left(\dot{\mu} \lambda_{\infty}-2 v \omega\right)\left(-\omega \tilde{\eta}_{1}-\tilde{\eta}_{3}+\beta \tilde{\eta}_{4} / 2\right)+N(U, \pi), \quad(1.8
\end{aligned}
$$

where we use $v(s)=\lambda(t(s)) / \lambda_{\infty}(s), \lambda(t(s))=\lambda(s), \dot{\lambda}=\frac{\partial}{\partial s} \lambda(s)$, and $N(U, \pi)$ is quadratic in $U$ but also depends on the modulation parameters $\lambda(s)$ etc., as well as the parameters at infinity $a_{\infty}$ etc. We denote the latter collectively as $\pi$, following the notation in [?], [KriSch1]. The operator $\mathcal{H}(s)$ in the preceding is given by

$$
\begin{aligned}
& \mathcal{H}(s):= \\
& \qquad\left(\begin{array}{cc}
\partial_{y}^{2}-1+3 v^{2}(s) \phi_{0}^{4}\left(\lambda\left(\mu_{\infty}-\mu+\lambda \lambda_{\infty}^{-1} y\right)\right) & 2 v^{2} \phi_{0}^{4}\left(\lambda\left(\mu_{\infty}-\mu+\lambda_{\infty}^{-1} y\right)\right) e^{2 i\left(\Psi-\Psi_{\infty}\right)} \\
-2 v^{2} \phi_{0}^{4}\left(\lambda\left(\mu_{\infty}-\mu+\lambda_{\infty}^{-1} y\right)\right) e^{-2 i\left(\Psi-\Psi_{\infty}\right)} & -\partial_{y}^{2}+1-3 v^{2}(s) \phi_{0}^{4}\left(\lambda\left(\mu_{\infty}-\mu+\lambda_{\infty}^{-1} y\right)\right)
\end{array}\right),
\end{aligned}
$$

where we use the notation $\Psi_{\infty}(s, y):=\tilde{\Psi}_{\infty}(s, y)+s$. The orthogonality relations 1.6 become the following:

$$
\left\langle\left(\begin{array}{l}
U \\
\bar{U}
\end{array}\right), \tilde{\xi}_{i}\right\rangle=0, \quad i=2, \ldots, 6
$$

and upon differentiating with respect to $s$ imply a set of ODE's for the parameters $\lambda(s)$ etc. The crux now is to deduce a priori estimates for the transformed radiation $\operatorname{part}\left(\begin{array}{c}U \\ U\end{array}\right)$ as well as for the modulation parameters; the latter need to satisfy the required asymptotic estimates for $s \rightarrow \infty$. In order to control the radiation part, one essentially 8 invokes a decomposition

$$
\left(\begin{array}{l}
U \\
\bar{U}
\end{array}\right)(s, y)=\left(\begin{array}{l}
U \\
\bar{U}
\end{array}\right)_{\mathrm{dis}}(s, y)+\sum_{i=1}^{6} \lambda_{i}(s) \eta_{i, \operatorname{proper}}(y),
$$

where the coefficients $\lambda_{i}(s), i=1, \ldots, 5$, are determined by the orthogonality relations (1.9). The coefficient $\lambda_{6}(s)$ is determined by means of the requirement $\lim _{s \rightarrow \infty} \lambda_{6}(s)=0$, which forces an initial condition $\lambda_{6}(0)$, similarly to the supercritical case treated in [Sch],

8 For technical reasons, one uses such a decomposition for a slightly transformed function $\left(\frac{\tilde{U}}{\tilde{U}}\right)$. 
[KriSch1]. By comparison to the latter, though, controlling $\lambda_{6}(s)$ appears more difficult, and requires the development of rather new technology. Specifically, a careful analysis of the modulation equations reveals that one needs to control quantities of the form $\int_{T}^{\infty} t \lambda_{6}(t) d t$, which upon substituting the solution for the ODE satisfied by $\lambda_{6}(t)$ results in quadratic expressions of at worst the form $9 \int_{T}^{\infty} t \int_{t}^{\infty}\left\langle U^{2}(s)-\bar{U}^{2}(s), \phi\right\rangle d s d t$ etc., where $\phi$ stands for a suitable Schwartz function. This shows that one should aim for a local decay of the radiation part of at least the strength $|\langle U(t, \cdot), \phi\rangle| \lesssim\langle t\rangle^{-3 / 2}$ in order to be able to estimate this expression; indeed, this local decay rate is in accordance with the linear estimates derived in [KriSch1]. However, we are dealing with a non-linear problem here. This is the first significant difficulty to be overcome:

Stage B: Deducing the strong local dispersive ${ }^{10}$ estimate for the radiation part

Schematically, the equation $(1.8)$ can be recast as

$$
\left(i \partial_{s}+\mathcal{H}\right)\left(\begin{array}{c}
U \\
\bar{U}
\end{array}\right)=V U+\left(\begin{array}{c}
|U|^{4} U \\
-|U|^{4} \bar{U}
\end{array}\right),
$$

where $\mathcal{H}=\left(\begin{array}{cc}\partial_{y}^{2}-1+3 \phi_{0}^{4}(\cdot, 1) & 2 \phi_{0}^{4}(\cdot, 1) \\ -2 \phi_{0}^{4}(\cdot, 1) & -\partial_{y}^{2}+1-3 \phi_{0}^{4}(\cdot, 1)\end{array}\right)$, and $V$, a Schwartz function, depends on $U$ as well as the modulation parameters etc. The loca ${ }^{11}$ expression $V U$ is due to interactions of the drifting soliton with itself as well as to interactions of the radiation with the drifting soliton, while the non-local quintilinear expressions $|U|^{4} U$ come from interactions of the radiation part with itself. While the root part of $\left(\begin{array}{c}U \\ U\end{array}\right)$ is controlled in terms of the coefficients $\lambda_{i}(s), i=1, \ldots, 6$, whose estimation is relegated to the third stage, the dispersive par ${ }^{12}$ (see the next section for the linear background) $(\underset{U}{U})_{\text {dis }}$ satisfies

$$
\left(i \partial_{s}+\mathcal{H}\right)\left(\begin{array}{c}
U \\
\bar{U}
\end{array}\right)_{\mathrm{dis}}=\left[V U+\left(\begin{array}{c}
|U|^{4} U \\
-|U|^{4} \bar{U}
\end{array}\right)\right]_{\mathrm{dis}} .
$$

The really difficult contribution on the right comes from the non-local quintilinear term: note that the standard way to deduce the local estimate is to combine the linear estimate ${ }^{13}$

$$
\left|\left\langle e^{i t \mathcal{H}} \phi_{\text {dis }}, \psi\right\rangle\right| \lesssim t^{-3 / 2}\|\langle x\rangle \phi\|_{L_{x}^{1}}\|\langle x\rangle \psi\|_{L_{x}^{1}}
$$

with Duhamel's formula, which then forces us to estimate the expression

$$
\int_{0}^{t}\langle t-s\rangle^{-3 / 2}\left\|\langle x\rangle|U|^{4}(s, x) U(s, x)\right\|_{L_{x}^{1}} d s .
$$

\footnotetext{
9 We are again careless here; the expression should really involve $\tilde{U}^{2}-\overline{\tilde{U}}^{2}$.

10 More precisely, we establish the strong local dispersive estimate up to an arbitrarily small error.

11 We refer to expressions which are Schwartz functions alternatively as 'local'.

12 Again, one should really use $\left(\frac{\tilde{U}}{\tilde{U}}\right)_{\text {dis }}$.

13 We abuse notation here and use letters $\phi, \psi$ to denote vector-valued functions. Also, we let $\langle x\rangle=|x|+1$.
} 
On the other hand, again from the linear theory summarized in the next section we expect the estimate

$$
\begin{aligned}
\left\|\langle x\rangle|U|^{4}(s, x) U(s, x)\right\|_{L_{x}^{1}} & \lesssim\|U(s, \cdot)\|_{L_{x}^{\infty}}^{3}\|x U(s, x)\|_{L_{x}^{2}}\|U(s, x)\|_{L_{x}^{2}} \\
& \lesssim\langle s\rangle^{-3 / 2} s=s^{-1 / 2},
\end{aligned}
$$

which only gives the decay $t^{-1 / 2}$ when substituted into $(1.10)$. One can modify this argument to eke out a local dispersive decay of $\langle t\rangle^{-1+}$, which however is insufficient for controlling the root part and modulation parameters.

The way out of this is to observe that the quintilinear expression exhibits a special algebraic cancellation structure, which in combination with the linear theory of $\mathcal{H}$ (and in particular the absence of resonances at the edges of the essential spectrum) allows one to significantly improve on the preceding. To explain the use of this algebraic structure heuristically, note that one expects the small-frequency part of $\left(\begin{array}{c}|U|^{4} U \\ -|U|^{4} \bar{U}\end{array}\right)_{\mathrm{dis}}$ to contribute less due to the absence of resonances at the end of the essential spectrum of $\mathcal{H}$. Another reason is that the small frequency part propagates more slowly, and hence when hit with a weight $\langle x\rangle$ should cost less than the $s$ used in the above calculation. On the other hand, assume that we localize one of the factors $|U|^{2}$ in $|U|^{4}=|U|^{2}|U|^{2}$ to relatively large frequency ${ }^{14}$ In that case the key is to use the following simple identity:

$$
2 i s \partial_{x}\left[|U|^{2}\right](s, \cdot)=\left(x+2 i s \partial_{x}\right) U(s, \cdot) \bar{U}(s, \cdot)-U(s, \cdot) \overline{\left(x+2 i s \partial_{x}\right) U(s, \cdot)} .
$$

The operator $C=x+2 i s \partial_{x}$ is the standard pseudo-conformal operator, and one expects an estimate $\sup _{s \geq 0}\left\|\left(x+2 i s \partial_{x}\right) U(s, \cdot)\right\|_{L_{x}^{2}} \lesssim 1$. Indeed, this turns out to be true (although establishing it requires a couple of tricks, as we do not deal with the free evolution $e^{i t \Delta}$ here). Thus provided we restrict the frequency of $|U|^{2}(s, \cdot)$ sufficiently far away from 0 , we expect to be able to score an extra gain here, and one can play these two considerations against each other to almost obtain the optimal estimate. This very crudely summarizes the strategy for stage B.

\section{Stage C: Controlling the root part of $\left(\frac{U}{U}\right)$ and the modulation parameters}

We now return to controlling $\lambda_{6}(t)$ as well as the modulation parameters, which we recall involved estimating expressions such as $\int_{T}^{\infty} t \int_{t}^{\infty}\left\langle U^{2}(s)-\bar{U}^{2}(s), \phi\right\rangle d s d t$, as well as similar ones. Clearly even the strong local dispersive estimate is not good enough for this purpose, and we have to resort to more refined considerations. In case of the above expression, this involves identifying another instance of an algebraic cancellation structure, in this case a symplectic structure. Again this shall rely on the spectral properties of $\mathcal{H}$.

\footnotetext{
14 By this we mean here frequency in the Littlewood-Paley sense. One has to be a bit careful to keep this separate from frequency in the sense of $\mathcal{H}$. The relation of the two will become clear thanks to the explicit distorted Fourier basis explained in the next section; the general heuristic is that a function with small frequency with respect to $\mathcal{H}$ is the sum of a (negligible) Schwartz function plus a function of small frequency in the Littlewood-Paley sense.
} 


\section{Stage D: Locating a fixed point}

The a priori estimates suggest running a Banach iteration; unfortunately, the presence of the phase $e^{i\left(\Psi-\Psi_{\infty}\right)(s, y)}$ with $\left(\Psi-\Psi_{\infty}\right)(s, y)$ growing like $s^{\frac{1}{2}+}$ does not allow one to deduce good estimates for the differences of iterates. This is the fundamental obstacle to proving Conjecture 1.2. We thus have to resort to an abstract fixed point theorem (Schauder-Tikhonov Theorem) to prove Theorem 1.3 It is to be hoped that the techniques developed in this paper help to further elucidate the nature of the non-generic blow-up solutions.

\section{Background material on $\mathcal{H}$}

The material in this section quickly summarizes certain facts established in the last section of [KriSch1], much of which was based on the work of Buslaev-Perelman and Perelman as well as earlier work by the second author. We refer to [KriSch1] as well as [Per] for proofs. Consider the operator

$$
\mathcal{H}=\left(\begin{array}{cc}
\partial_{x}^{2}-1+3 \phi_{0}^{4}(x, 1) & 2 \phi_{0}^{4}(x, 1) \\
-2 \phi_{0}^{4}(x, 1) & -\partial_{x}^{2}+1-3 \phi_{0}^{4}(x, 1)
\end{array}\right) .
$$

Its spectrum is $(-\infty,-1] \cup[1, \infty) \cup\{0\}$, with essential spectrum $(-\infty,-1] \cup[1, \infty)$ and discrete spectrum $\{0\}$ of geometric multiplicity 2 and algebraic multiplicity 6 . The generalized root space $\mathcal{N}$ is generated by the following vector-valued functions (from now on, we adhere to the convention $\phi_{0}:=\phi_{0}(\cdot, 1)$, see the preceding section):

$$
\begin{aligned}
\eta_{1, \text { proper }}(z):=\left(\begin{array}{c}
i \phi_{0}(z) \\
-i \phi_{0}(z)
\end{array}\right), & \eta_{2, \text { proper }}(z):=\left(\begin{array}{c}
z \phi_{0}^{\prime}(z)+\phi_{0}(z) / 2 \\
z \phi_{0}^{\prime}(z)+\phi_{0}(z) / 2
\end{array}\right), \\
\eta_{3, \text { proper }}(z):=\left(\begin{array}{c}
\phi_{0}^{\prime}(z) \\
\phi_{0}^{\prime}(z)
\end{array}\right), & \eta_{4, \text { proper }}(z):=\left(\begin{array}{c}
i z \phi_{0}(z) \\
-i z \phi_{0}(z)
\end{array}\right), \\
\eta_{5, \text { proper }}(z):=\left(\begin{array}{c}
i z^{2} \phi_{0}(z) \\
-i z^{2} \phi_{0}(z)
\end{array}\right), & \eta_{6, \text { proper }}(z):=\left(\begin{array}{c}
\rho(z) \\
\rho(z)
\end{array}\right) .
\end{aligned}
$$

The first five are in one-one correspondence with internal symmetries ('good modes'), while the last is the 'exotic mode', characterized by

$$
L_{+} \rho=z^{2} \phi_{0}(z), \quad L_{+}=-\partial_{x}^{2}+1-5 \phi_{0}^{4} .
$$

The root space is generated by $\eta_{1 \text {,proper, }}, \eta_{3, \text { proper }}$.

As for the essential spectrum, its edges \pm 1 are no resonances. This means that there are no non-zero solutions $f_{ \pm} \in L^{\infty}$ satisfying

$$
\mathcal{H} f_{ \pm}= \pm f_{ \pm}
$$

This is in marked contrast to the operator $\mathcal{H}_{0}:=\left(\begin{array}{cc}\partial_{x}^{2}-1 & 0 \\ 0 & -\partial_{x}^{2}+1\end{array}\right)$, and responsible for much improved local decay estimates. 
Identical observations apply to the operator

$$
\mathcal{H}^{*}=\left(\begin{array}{cc}
\partial_{x}^{2}-1+3 \phi_{0}^{4}(x, 1) & -2 \phi_{0}^{4}(x, 1) \\
+2 \phi_{0}^{4}(x, 1) & -\partial_{x}^{2}+1-3 \phi_{0}^{4}(x, 1)
\end{array}\right),
$$

its generalized root space $\mathcal{N}^{*}$ being generated by

$$
\begin{aligned}
& \xi_{1, \operatorname{proper}}(z):=\left(\begin{array}{c}
\phi_{0}(z) \\
\phi_{0}(z)
\end{array}\right), \quad \xi_{2, \operatorname{proper}}(z):=\left(\begin{array}{c}
i\left(z \phi_{0}^{\prime}(z)+\phi_{0}(z) / 2\right) \\
-i\left(z \phi_{0}^{\prime}(z)+\phi_{0}(z) / 2\right)
\end{array}\right), \\
& \xi_{3, \operatorname{proper}}(z):=\left(\begin{array}{c}
i \phi_{0}^{\prime}(z) \\
-i \phi_{0}^{\prime}(z)
\end{array}\right), \quad \xi_{4, \operatorname{proper}}(z):=\left(\begin{array}{c}
z \phi_{0}(z) \\
z \phi_{0}(z)
\end{array}\right) \text {, } \\
& \xi_{5, \operatorname{proper}}(z):=\left(\begin{array}{c}
z^{2} \phi_{0}(z) \\
z^{2} \phi_{0}(z)
\end{array}\right), \quad \xi_{6, \text { proper }}(z):=\left(\begin{array}{c}
i \rho(z) \\
-i \rho(z)
\end{array}\right) .
\end{aligned}
$$

Then we have the direct sum decomposition

$$
L^{2}(\mathbb{R}) \times L^{2}(\mathbb{R})=\mathcal{N}+\left(\mathcal{N}^{*}\right)^{\perp} .
$$

This means that every vector-valued function $(\underset{U}{U})(x)$ with $U(\cdot) \in L^{2}(\mathbb{R})$ can be uniquely represented as

$$
\left(\begin{array}{l}
U \\
\bar{U}
\end{array}\right)=\sum_{i=1}^{6} \lambda_{i} \eta_{i, \text { proper }}+\left(\begin{array}{l}
U \\
\bar{U}
\end{array}\right)_{\mathrm{dis}}, \quad\left(\begin{array}{l}
U \\
\bar{U}
\end{array}\right)_{\mathrm{dis}} \in\left(\mathcal{N}^{*}\right)^{\perp} .
$$

In order to determine the $\lambda_{i}$, one uses the following table of orthogonality relations 15

$$
\begin{array}{ll}
\left\langle\eta_{j, \text { proper }}, \xi_{1, \text { proper }}\right\rangle=0,1 \leq j \leq 5, & \left\langle\eta_{6, \text { proper }}, \xi_{1, \text { proper }}\right\rangle=2 \kappa_{2}, \\
\left\langle\eta_{j, \text { proper }}, \xi_{2, \text { proper }}\right\rangle=0, j=1,2,3,4,6, & \left\langle\eta_{5, \text { proper }}, \xi_{2, \text { proper }}\right\rangle=-4 \kappa_{2}, \\
\left\langle\eta_{j, \text { proper }}, \xi_{3, \text { proper }}\right\rangle=0, j=1,2,3,5,6, & \left\langle\eta_{4, \text { proper }}, \xi_{3, \text { proper }}\right\rangle=-\kappa_{1}, \\
\left\langle\eta_{j, \text { proper }}, \xi_{4, \text { proper }}\right\rangle=0, j=1,2,4,5,6, & \left\langle\eta_{3, \text { proper }}, \xi_{4, \text { proper }}\right\rangle=-\kappa_{1}, \\
\left\langle\eta_{j, \text { proper }}, \xi_{5, \text { proper }}\right\rangle=0, j=1,3,4,5, & \left\langle\eta_{2, \text { proper }}, \xi_{5, \text { proper }}\right\rangle=-4 \kappa_{2}, \\
\left\langle\eta_{6, \text { proper }}, \xi_{5, \text { proper }}\right\rangle=2 \kappa_{3}, & \\
\left\langle\eta_{j, \text { proper }}, \xi_{6, \text { proper }}\right\rangle=0, j=2,3,4,6, & \left\langle\eta_{1, \text { proper }}, \xi_{6, \text { proper }}\right\rangle=2 \kappa_{2}, \\
\left\langle\eta_{5, \text { proper }}, \xi_{6, \text { proper }}\right\rangle=2 \kappa_{3}, &
\end{array}
$$

where we use the notation

$$
\left\langle\phi_{0}, \phi_{0}\right\rangle=\kappa_{1}>0, \quad\left\langle\rho, \phi_{0}\right\rangle=\frac{1}{2} \int x^{2} \phi_{0}^{2}(x) d x=: \kappa_{2}>0, \quad\left\langle x^{2} \phi_{0}, \rho\right\rangle=: \kappa_{3} .
$$

We also write

$$
\sum_{i=1}^{6} \lambda_{i} \eta_{i, \text { proper }}=P_{\text {root }}\left(\begin{array}{l}
U \\
\bar{U}
\end{array}\right), \quad\left(\begin{array}{l}
U \\
\bar{U}
\end{array}\right)_{\text {dis }}=P_{s}\left(\begin{array}{l}
U \\
\bar{U}
\end{array}\right) .
$$

We have the following important linear estimates:

15 We use the convention $\left\langle\left(\begin{array}{c}U_{1} \\ U_{2}\end{array}\right),\left(\begin{array}{l}V_{1} \\ V_{2}\end{array}\right)\right\rangle=\left\langle U_{1}, V_{1}\right\rangle+\left\langle U_{2}, V_{2}\right\rangle$. 
Theorem 2.1. The following estimates hold for vector-valued functions $f(\cdot) \in L^{1}(\mathbb{R}) \cap$ $L^{2}(\mathbb{R})$ and $0 \leq \theta \leq 1$ :

$$
\begin{aligned}
& \left\|e^{i t \mathcal{H}} P_{S} f\right\|_{L^{2}} \lesssim\|f\|_{L^{2}}, \quad\left\|e^{i t \mathcal{H}} P_{S} f\right\|_{L^{\infty}} \lesssim|t|^{-1 / 2}\|f\|_{L^{1}}, \\
& \left\|\langle x\rangle^{-\theta} e^{i t \mathcal{H}} P_{S} f\right\|_{L^{\infty}} \lesssim|t|^{-1 / 2-\theta \mid}\|\langle x\rangle f\|_{L_{x}^{1}} .
\end{aligned}
$$

The first two of these are just as for $e^{i t \Delta}$, while the last is not true for the latter and due to the absence of resonances at the edges of the essential spectrum of $\mathcal{H}$.

By analogy to Fourier transformation associated with $\Delta$, there is a distorted Fourier transform associated with $\mathcal{H}$ :

Theorem 2.2. There exist Lipschitz continuous vector-valued functions $e_{ \pm}(x, \xi)$ with the property

$$
P_{S}\left(\begin{array}{l}
U \\
\bar{U}
\end{array}\right)(x)=\sum_{ \pm} \int_{-\infty}^{\infty} e_{ \pm}(x, \xi)\left\langle\left(\begin{array}{l}
U \\
\bar{U}
\end{array}\right), \sigma_{3} e_{ \pm}(x, \xi)\right\rangle d \xi, \quad \sigma_{3}=\left(\begin{array}{cc}
1 & 0 \\
0 & -1
\end{array}\right)
$$

for every rapidly ${ }^{16}$ decaying function $x \mapsto(\underset{U}{U})(x)$. Moreover, there exist smooth functions $s(\xi), r(\xi)$ satisfying $s(0)=0, r(0)=-1$, as well as suitable numbers $\gamma, \mu>0$ such that

$e_{+}(x, \xi)= \begin{cases}s(\xi)\left[e^{i x \xi}\left(\begin{array}{l}1 \\ 0\end{array}\right)+O\left((1+|\xi|)^{-1} e^{-\gamma x}\right)\right] & \\ +O\left(\xi(1+|\xi|)^{-2} e^{-\mu x}\right), & (x, \xi) \in \mathbb{R}_{\geq 0} \times \mathbb{R}_{\geq 0}, \\ {\left[e^{i x \xi}+r(\xi) e^{-i x \xi}\right]\left(\begin{array}{l}1 \\ 0\end{array}\right)+O\left(\xi(1+|\xi|)^{-2} e^{\gamma x}\right),} & (x, \xi) \in \mathbb{R}_{<0} \times \mathbb{R}_{\geq 0}, \\ s(-\xi)\left[e^{i x \xi}\left(\begin{array}{l}1 \\ 0\end{array}\right)+O\left((1+|\xi|)^{-1} e^{+\gamma x}\right)\right] & \\ +O\left(\xi(1+|\xi|)^{-2} e^{+\mu x}\right), & (x, \xi) \in \mathbb{R}_{<0} \times \mathbb{R}_{<0}, \\ {\left[e^{i x \xi}+r(-\xi) e^{-i x \xi}\right]\left(\begin{array}{l}1 \\ 0\end{array}\right)+O\left(\xi(1+|\xi|)^{-2} e^{-\gamma x}\right),} & (x, \xi) \in \mathbb{R}_{\geq 0} \times \mathbb{R}_{<0} .\end{cases}$

Also, we have the relation

$$
e_{-}(x, \xi)=\sigma_{1} e_{+}(x, \xi), \quad \sigma_{1}=\left(\begin{array}{ll}
0 & 1 \\
1 & 0
\end{array}\right) .
$$

In analogy to the usual Fourier transform, there is a distorted Plancherel's Theorem:

Theorem 2.3. Let $\phi, \psi \in \mathcal{S}(\mathbb{R})$ be vector-valued functions. Then

$$
\left\langle P_{s} \phi, \psi\right\rangle=\sum_{ \pm} \frac{1}{2 \pi} \int_{-\infty}^{\infty}\left\langle\phi, \sigma_{3} e_{ \pm}(\cdot, \xi)\right\rangle \overline{\left\langle\psi, e_{ \pm}(\cdot, \xi)\right\rangle} d \xi
$$

\footnotetext{
16 We are being overly restrictive in the formulation here; all facts about the distorted Fourier transform apply in the same degree of generality as for the ordinary Fourier transform.
} 
We shall use the notation

$$
\mathcal{F}_{ \pm}(\phi)(\xi):=\left\langle\phi, \sigma_{3} e_{ \pm}(\cdot, \xi)\right\rangle, \quad \tilde{\mathcal{F}}_{ \pm}(\phi)(\xi):=\left\langle\phi, e_{ \pm}(x, \xi)\right\rangle
$$

When working with $e_{ \pm}(x, \xi)$, we shall for example write

$$
e_{+}(x, \xi)=s(\xi) e^{i x \xi} \underline{e}+\phi(x, \xi), \quad(x, \xi) \in \mathbb{R}_{\geq 0} \times \mathbb{R}_{\geq 0},
$$

and similarly for the other values of $(x, \xi)$. The functions $\phi(x, \xi)$, which are Schwartz with respect to $x$, are understood to vary accordingly, but all vanish uniformly in $x$ at $\xi=0$ and decay like $|\xi|^{-1}$ for $|\xi| \rightarrow \infty$ uniformly in $x$.

\section{Setting up the equations}

\subsection{Algebraic manipulations I. Passage to a different reference frame}

We now flesh out the discussion of the first section. In this section as well as the next, we shall use formal algebraic manipulations to derive the equations which will serve to define the iterative step. We shall also mention the required estimates. In the final sections of the paper, we shall show that the iterative step indeed makes sense when performed on suitable function spaces. Thus consider now a solution $\psi(t, x)=W(t, x)+R(t, x)$ of (1.1), where

$$
W(t, x)=e^{i \theta(t, x)} e^{-i \frac{\beta(t)}{4} \lambda^{2}(t)(x-\mu(t))^{2}} \sqrt{\lambda(t)} \phi_{0}(\lambda(t)(x-\mu(t))) .
$$

Use the notation $\Psi(t, x)=\theta(t, x)-\frac{\beta}{4} \lambda^{2}(x-\mu)^{2}, z=\lambda(x-\mu)$. Also, write $\tilde{\theta}(t, z):=$ $\theta(t, x)$. An elementary calculation then shows that

$$
\begin{aligned}
i \partial_{t} W+\partial_{x}^{2} W+|W|^{4} W & =\left(\lambda_{t} \lambda^{-1}-\beta \lambda^{2}\right)\left[i e^{i \Psi} z \lambda^{1 / 2} \phi_{0}^{\prime}(z)+\frac{\beta}{2} z^{2} W+\frac{i}{2} W-\tilde{\theta}_{z} z W\right] \\
& +\frac{z^{2}}{4}\left(\beta_{t}+\lambda^{2} \beta^{2}\right) W+i \lambda^{2} \tilde{\theta}_{z z} W+\left(\lambda^{2}-\tilde{\theta}_{t}+\lambda^{2} \tilde{\theta}_{z}^{2}-\beta \lambda^{2} z \tilde{\theta}_{z}\right) W \\
& +\left(\mu_{t}-2 \lambda \tilde{\theta}_{z}\right)\left[\lambda \tilde{\theta}_{z}-i \lambda^{3 / 2} e^{i \Psi} \phi_{0}^{\prime}(z)-\frac{\beta}{2} \lambda z W\right]
\end{aligned}
$$

Introduce the following notation:

$$
\begin{aligned}
\eta_{1}:=\left(\begin{array}{c}
i e^{i \Psi} \sqrt{\lambda} \phi_{0}(z) \\
-i e^{-i \Psi} \sqrt{\lambda} \phi_{0}(z)
\end{array}\right), & \eta_{2}:=\left(\begin{array}{c}
e^{i \Psi}\left(z \sqrt{\lambda} \phi_{0}^{\prime}(z)+\sqrt{\lambda} \phi_{0}(z) / 2\right) \\
e^{-i \Psi}\left(z \sqrt{\lambda} \phi_{0}^{\prime}(z)+\sqrt{\lambda} \phi_{0}(z) / 2\right)
\end{array}\right), \\
\eta_{3}:=\left(\begin{array}{c}
e^{i \Psi} \sqrt{\lambda} \phi_{0}^{\prime}(z) \\
e^{-i \Psi} \sqrt{\lambda} \phi_{0}^{\prime}(z)
\end{array}\right), & \eta_{4}:=\left(\begin{array}{c}
i e^{i \Psi} z \sqrt{\lambda} \phi_{0}(z) \\
-i e^{-i \Psi} z \sqrt{\lambda} \phi_{0}(z)
\end{array}\right), \\
\eta_{5}:=\left(\begin{array}{c}
i e^{i \Psi} z^{2} \sqrt{\lambda} \phi_{0}(z) \\
-i e^{-i \Psi} z^{2} \sqrt{\lambda} \phi_{0}(z)
\end{array}\right), & \eta_{6}:=\left(\begin{array}{c}
e^{i \Psi} \sqrt{\lambda} \rho(z) \\
e^{-i \Psi} \sqrt{\lambda} \rho(z)
\end{array}\right) .
\end{aligned}
$$


Now impose the relation $\tilde{\theta}_{z z}=0$, whence $\tilde{\theta}=\gamma(t)+\omega(t) z$. The function $\rho$ here is defined via

$$
\begin{gathered}
L_{-}:=-\partial_{x x}+1-\phi_{0}^{4}, \quad L_{+}:=-\partial_{x x}+1-5 \phi_{0}^{4}, \\
L_{-} \phi_{0}=0, \quad L_{+}\left(\frac{1}{2} \phi_{0}+x \phi_{0}^{\prime}\right)=-2 \phi_{0}, \\
L_{-}\left(x^{2} \phi_{0}\right)=-4\left(\frac{1}{2} \phi_{0}+x \phi_{0}^{\prime}\right), \quad L_{+} \rho=x^{2} \phi_{0} .
\end{gathered}
$$

Then the vector-valued function $\mathcal{W}(t, x):=\left(\begin{array}{c}W(t, x) \\ \bar{W}(t, x)\end{array}\right)$ satisfies

$$
\begin{aligned}
i \partial_{t} \mathcal{W}+ & {\left[\begin{array}{cc}
\partial_{x x} & 0 \\
0 & -\partial_{x x}
\end{array}\right] \mathcal{W}+\left(\begin{array}{c}
|W|^{4} W \\
-|W|^{4} \bar{W}
\end{array}\right) } \\
= & i\left(\dot{\lambda} \lambda^{-1}-\beta \lambda^{2}\right)\left(\eta_{2}-\beta \eta_{5} / 2+\omega \eta_{4}\right)-\frac{i}{4}\left(\dot{\beta}+\lambda^{2} \beta^{2}\right) \eta_{5}-i\left(\lambda^{2}-\dot{\gamma}+\lambda^{2} \omega^{2}\right) \eta_{1} \\
& +i\left(\dot{\omega}+\beta \lambda^{2} \omega\right) \eta_{4}+\lambda(\dot{\mu}-2 \lambda \omega)\left(-i \omega \eta_{1}-i \eta_{3}+i \beta \eta_{4} / 2\right)
\end{aligned}
$$

Write $\left(\begin{array}{c}\psi(t, x) \\ \bar{\psi}(t, x)\end{array}\right)=\mathcal{W}(t, x)+Z(t, x)$ where $Z(t, x)=\left(\begin{array}{l}R(t, x) \\ \bar{R}(t, x)\end{array}\right)$. We deduce the following equation for $Z$ :

$$
\begin{aligned}
i \partial_{t} Z+\mathcal{H}(t) Z= & -i\left(\dot{\lambda} \lambda^{-1}-\beta \lambda^{2}\right)\left(\eta_{2}-\beta \eta_{5} / 2+\omega \eta_{4}\right) \\
& +\frac{i}{4}\left(\dot{\beta}+\lambda^{2} \beta^{2}\right) \eta_{5}+i\left(\lambda^{2}-\dot{\gamma}+\lambda^{2} \omega^{2}\right) \eta_{1} \\
& -i\left(\dot{\omega}+\beta \lambda^{2} \omega\right) \eta_{4}-\lambda(\dot{\mu}-2 \lambda \omega)\left(-i \omega \eta_{1}-i \eta_{3}+i \beta \eta_{4} / 2\right)+N(Z), \\
\mathcal{H}(t):= & {\left[\begin{array}{cc}
\partial_{x x}+3|W|^{4} & 2|W|^{2} W^{2} \\
-2|W|^{2} \bar{W}^{2} & -\partial_{x x}-3|W|^{4}
\end{array}\right], } \\
N(Z):= & \left(\begin{array}{c}
-|R+W|^{4}(R+W)+|W|^{4} W+3|W|^{4} R+2|W|^{2} W^{2} \bar{R} \\
|R+W|^{4}(\bar{R}+\bar{W})-|W|^{4} \bar{W}-3|W|^{4} \bar{R}-2|W|^{2} \bar{W}^{2} R
\end{array}\right) \\
= & \left(\begin{array}{c}
-3 R^{2}|W|^{2} \bar{W}-6|R|^{2}|W|^{2} W-W^{3} \bar{R}^{2}+O\left(|R|^{3}|W|^{2}+|R|^{5}\right) \\
3 \bar{R}^{2}|W|^{2} W+6|R|^{2}|W|^{2} \bar{W}+\bar{W}^{3} R^{2}+O\left(|R|^{3}|W|^{2}+|R|^{5}\right)
\end{array}\right),
\end{aligned}
$$

In order to formulate the modulation equations, it will be convenient to introduce the following family of auxiliary functions, which are in some sense dual to the $\eta_{i}$ :

$$
\begin{array}{ll}
\xi_{1}:=\left(\begin{array}{c}
e^{i \Psi} \sqrt{\lambda} \phi_{0}(z) \\
e^{-i \Psi} \sqrt{\lambda} \phi_{0}(z)
\end{array}\right), & \xi_{2}:=\left(\begin{array}{c}
i e^{i \Psi}\left(z \sqrt{\lambda} \phi_{0}^{\prime}(z)+\sqrt{\lambda} \phi_{0}(z) / 2\right) \\
-i e^{-i \Psi}\left(z \sqrt{\lambda} \phi_{0}^{\prime}(z)+\sqrt{\lambda} \phi_{0}(z) / 2\right)
\end{array}\right), \\
\xi_{3}:=\left(\begin{array}{c}
i e^{i \Psi} \sqrt{\lambda} \phi_{0}^{\prime}(z) \\
-i e^{-i \Psi} \sqrt{\lambda} \phi_{0}^{\prime}(z)
\end{array}\right), & \xi_{4}:=\left(\begin{array}{c}
e^{i \Psi} z \sqrt{\lambda} \phi_{0}(z) \\
e^{-i \Psi} z \sqrt{\lambda} \phi_{0}(z)
\end{array}\right), \\
\xi_{5}:=\left(\begin{array}{c}
e^{i \Psi} z^{2} \sqrt{\lambda} \phi_{0}(z) \\
e^{-i \Psi} z^{2} \sqrt{\lambda} \phi_{0}(z)
\end{array}\right), & \xi_{6}:=\left(\begin{array}{c}
i e^{i \Psi} \sqrt{\lambda} \rho(z) \\
-i e^{-i \Psi} \sqrt{\lambda} \rho(z)
\end{array}\right) .
\end{array}
$$


Then, analogously to 2.2, we have the following (using the same notation):

$$
\begin{array}{lll}
\left\langle\eta_{j}, \xi_{1}\right\rangle=0,1 \leq j \leq 5, & \left\langle\eta_{6}, \xi_{1}\right\rangle=2 \kappa_{2}, \\
\left\langle\eta_{j}, \xi_{2}\right\rangle=0, \quad j=1,2,3,4,6, & \left\langle\eta_{5}, \xi_{2}\right\rangle=-4 \kappa_{2}, & \\
\left\langle\eta_{j}, \xi_{3}\right\rangle=0, j=1,2,3,5,6, & \left\langle\eta_{4}, \xi_{3}\right\rangle=-\kappa_{1}, \\
\left\langle\eta_{j}, \xi_{4}\right\rangle=0, j=1,2,4,5,6, & \left\langle\eta_{3}, \xi_{4}\right\rangle=-\kappa_{1}, \\
\left\langle\eta_{j}, \xi_{5}\right\rangle=0, j=1,3,4,5, & \left\langle\eta_{2}, \xi_{5}\right\rangle=-4 \kappa_{2}, & \left\langle\eta_{6}, \xi_{5}\right\rangle=2 \kappa_{3}, \\
\left\langle\eta_{j}, \xi_{6}\right\rangle=0, j=2,3,4,6, & \left\langle\eta_{1}, \xi_{6}\right\rangle=2 \kappa_{2}, & \left\langle\eta_{5}, \xi_{6}\right\rangle=2 \kappa_{3} .
\end{array}
$$

Recall from the discussion in the first section that we impose the orthogonality relations $\left\langle Z, \xi_{i}\right\rangle=0, i=2, \ldots, 6$. This allows us to control the 'good component' of the root part of the radiation. Using Leibniz' rule we get

$$
\left\langle i \partial_{t} Z+\mathcal{H}(t) Z, \xi_{j}(t)\right\rangle=\left\langle Z,\left(i \partial_{t}+\mathcal{H}(t)^{*}\right) \xi_{j}(t)\right\rangle=:\left\langle Z, \mathcal{L}^{*} \xi_{j}\right\rangle
$$

Explicitly, using (3.1), these relations read as follows:

$$
\begin{aligned}
& -2 i \beta\left(\dot{\lambda} \lambda^{-1}-\beta \lambda^{2}\right) \kappa_{2}-i\left(\dot{\beta}+\lambda^{2} \beta^{2}\right) \kappa_{2}=\left\langle Z, \mathcal{L}^{*} \xi_{2}\right\rangle-\left\langle N(Z), \xi_{2}\right\rangle, \\
& \frac{i}{2} \lambda \beta \kappa_{1}(\dot{\mu}-2 \lambda \omega)+i \omega \kappa_{1}\left(\dot{\lambda} \lambda^{-1}-\beta \lambda^{2}\right)+i \kappa_{1}\left(\dot{\omega}+\beta \lambda^{2} \omega\right)=\left\langle Z, \mathcal{L}^{*} \xi_{3}\right\rangle-\left\langle N(Z), \xi_{3}\right\rangle, \\
& -i \lambda \kappa_{1}(\dot{\mu}-2 \lambda \omega)=\left\langle Z, \mathcal{L}^{*} \xi_{4}\right\rangle-\left\langle N(Z), \xi_{4}\right\rangle, \\
& 4 i \kappa_{2}\left(\dot{\lambda} \lambda^{-1}-\beta \lambda^{2}\right)=\left\langle Z, \mathcal{L}^{*} \xi_{5}\right\rangle-\left\langle N(Z), \xi_{5}\right\rangle, \\
& 2 i \kappa_{2}\left(\lambda^{2}-\dot{\gamma}+\lambda^{2} \omega^{2}\right)+2 i \lambda \omega \kappa_{2}(\dot{\mu}-2 \lambda \omega)+i \beta \kappa_{3}\left(\dot{\lambda} \lambda^{-1}-\beta \lambda^{2}\right)+\frac{i}{2} \kappa_{3}\left(\dot{\beta}+\lambda^{2} \beta^{2}\right) \\
& =\left\langle Z, \mathcal{L}^{*} \xi_{6}\right\rangle-\left\langle N(Z), \xi_{6}\right\rangle .
\end{aligned}
$$

Before proceeding, let us carry out a consistency check: we know that the case $Z=0$ corresponds to a transformed standing wave. In this case, the above relations simplify to

$$
\begin{aligned}
& \dot{\lambda} \lambda^{-1}-\beta \lambda^{2}=0, \quad \dot{\beta}+\lambda^{2} \beta^{2}=0, \quad \frac{d}{d t}(\lambda \beta)=0, \beta=-b \lambda^{-1}, \\
& \dot{\lambda}=-b \lambda^{2}, \quad \lambda(t)=(a+b t)^{-1}, \quad \beta(t)=-b(a+b t), \\
& \dot{\mu}-2 \lambda \omega=0, \quad \dot{\omega}+\beta \lambda^{2} \omega=0, \quad \dot{\omega}-b \lambda \omega=0, \\
& \omega(t)=v(a+b t), \quad \dot{\mu}=2 \lambda \omega=2 v, \quad \mu(t)=2 t v+\mu_{0}, \\
& \lambda^{2}-\dot{\gamma}+\lambda^{2} \omega^{2}=0, \quad \dot{\gamma}=\lambda^{2}+v^{2}=-\frac{1}{b} \dot{\lambda}+v^{2}, \\
& \gamma(t)=-\frac{\lambda}{b}+t v^{2}+\gamma_{0}=-\frac{1}{b(a+b t)}+v^{2} t+\gamma_{0} .
\end{aligned}
$$

So the exact solution looks as follows (with $\gamma=\gamma_{0}-v \mu_{0}$ ):

$$
\begin{aligned}
\theta(t, z) & =-\frac{1}{b(a+b t)}-v^{2} t+\gamma+v x, & & \beta(t)=-b(a+b t), \\
\lambda(t) & =(a+b t)^{-1}, & \mu(t) & =2 t v+\mu_{0},
\end{aligned}
$$


and the transformed standing wave $W$ is

$$
\begin{aligned}
W(t, x)= & \exp \left(i\left[-\frac{1}{b(a+b t)}-v^{2} t+\gamma+v x+\frac{b}{4(a+b t)}\left(x-2 t v-\mu_{0}\right)^{2}\right]\right) \\
& \cdot(a+b t)^{-1 / 2} \phi_{0}\left((a+b t)^{-1}\left(x-2 t v-\mu_{0}\right)\right) .
\end{aligned}
$$

Now recall the pseudo-conformal transformation

$$
\mathcal{C}_{M}: \psi(t, x) \mapsto(a+b t)^{-1 / 2} \exp \left(i \frac{b x^{2}}{4(a+b t)}\right) \psi\left(\frac{c+d t}{a+b t}, \frac{x}{a+b t}\right)
$$

where $M=\left(\begin{array}{ll}a & b \\ c & d\end{array}\right) \in S L(2, \mathbb{R})$. Starting from the standing wave $e^{i t} \phi_{0}(x)$, apply the pseudo-conformal transformation with matrix $\left(\begin{array}{cc}a & b \\ -b^{-1} & 0\end{array}\right)$ :

$$
\exp \left(-i \frac{1}{b(a+b t)}+i \frac{b}{4(a+b t)} x^{2}\right)(a+b t)^{-1 / 2} \phi_{0}\left((a+b t)^{-1} x\right)
$$

and then the Galilei transform

$$
\mathfrak{g}_{\gamma, v, \mu_{0}}(t)=e^{i\left(\gamma+v x-t v^{2}\right)} e^{-i\left(2 t v+\mu_{0}\right) p} .
$$

This leads to the exact same expression as in 3.3.

We now intend to translate the above equations from the $(t, x)$ coordinates to a new coordinate system $(s, y)$, in which we 'desingularize' the equations. The blow-up time $t_{*}$ shall be transformed into $s=+\infty$, and the $t$-interval $\left(-\infty, t_{*}\right]$ shall correspond to $(-c, \infty]$ for suitable $c>0$. Thus make the Ansat ${ }^{17}$

$$
\left(\begin{array}{l}
U \\
\bar{U}
\end{array}\right):=\mathcal{M} \mathcal{T}_{\infty}\left(\begin{array}{l}
R \\
\bar{R}
\end{array}\right)
$$

where

$$
\begin{aligned}
& \mathcal{T}_{\infty}=e^{-i\left(v_{\infty}^{2} s+\gamma_{\infty}+v_{\infty} y\right)} e^{i\left(2 v_{\infty} s+y_{\infty}\right) p}\left(\begin{array}{cc}
a_{\infty} & b_{\infty} \\
0 & a_{\infty}^{-1}
\end{array}\right), \\
& \mathcal{M}=\mathcal{M}(s)=\left(\begin{array}{cc}
e^{-i s} & 0 \\
0 & e^{i s}
\end{array}\right), \quad p=-i \frac{d}{d y} .
\end{aligned}
$$

Then we have the succinct identity

$$
\left(\mathcal{T}_{\infty} F\right)(s, y)=e^{-i \tilde{\Psi}_{\infty}(s, y)} \lambda_{\infty}^{-1 / 2}(s) F\left(\int_{0}^{s} \lambda_{\infty}^{-2}(\sigma) d \sigma, \lambda_{\infty}^{-1}(s) y+\mu_{\infty}(s)\right),
$$

where

$$
\begin{aligned}
& \tilde{\Psi}_{\infty}(s, y)=v_{\infty}^{2} s+\gamma_{\infty}+v_{\infty} y-\frac{b_{\infty}\left(y+2 v_{\infty} s+y_{\infty}\right)^{2}}{4\left(a_{\infty}+b_{\infty} s\right)} \\
& \mu_{\infty}(s)=\frac{2 v_{\infty} s+y_{\infty}}{a_{\infty}+b_{\infty} s}, \quad \lambda_{\infty}=a_{\infty}+b_{\infty} s
\end{aligned}
$$

\footnotetext{
17 We use the notation $U(s, y), U(s), U$ all for the same function of two variables in order to streamline the notation in some places.
} 
Thus we have

$$
\begin{aligned}
e^{-i s}\left(\mathcal{T}_{\infty} W\right)(s, y)= & e^{-i\left(s+\tilde{\Psi}_{\infty}\right)+i \Psi\left(t(s), \mu_{\infty}+\lambda_{\infty}^{-1}(s) y\right)} \lambda_{\infty}^{-1 / 2}(s) \lambda(t(s))^{1 / 2} \\
& \cdot \phi_{0}\left(\lambda(t(s))\left(\mu_{\infty}(s)-\mu(t(s))+\lambda_{\infty}^{-1}(s) y\right)\right),
\end{aligned}
$$

where we put $t(s):=\int_{0}^{s} \lambda_{\infty}^{-2}(\sigma) d \sigma=a_{\infty}^{-1} s /\left(a_{\infty}+b_{\infty} s\right)$. We shall now impose the asymptotic conditions $\nu(s):=\lambda(t(s)) / \lambda_{\infty}(s) \rightarrow 1, \lambda(t(s))\left(\mu_{\infty}(s)-\mu(t(s))\right) \rightarrow 0$ as $s \rightarrow+\infty$. Unfortunately, it appears that no such requirement can be applied to $\Psi\left(t(s), \mu_{\infty}+\lambda_{\infty}^{-1}(s) y\right)-s-\tilde{\Psi}_{\infty}(s, y)$, as will follow from the ensuing discussion. We shall write $\lambda(t(s))=: \lambda(s)$ etc. in the future.

Now introduce the Schwartz functions $\tilde{\eta}_{i}:=\mathcal{M} \mathcal{T}_{\infty} \eta_{i}, \tilde{\xi}_{i}:=\mathcal{M} \mathcal{T}_{\infty} \xi_{i}$. Then we can deduce the following equation for $\left(\begin{array}{c}U \\ \bar{U}\end{array}\right)$ :

$$
\begin{aligned}
i \partial_{s}\left(\begin{array}{c}
U \\
\bar{U}
\end{array}\right)+\mathcal{H}(s)\left(\begin{array}{c}
U \\
\bar{U}
\end{array}\right)=-i\left(\dot{\lambda} \lambda^{-1}-\beta v^{2}\right)\left(\tilde{\eta}_{2}-\beta \tilde{\eta}_{5} / 2+\omega \tilde{\eta}_{4}\right) \\
\quad+\frac{i}{4}\left(\dot{\beta}+\beta^{2} v^{2}\right) \tilde{\eta}_{5}+i\left(\nu^{2}-\dot{\gamma}+v^{2} \omega^{2}\right) \tilde{\eta}_{1} \\
\quad-i\left(\dot{\omega}+\beta \omega v^{2}\right) \tilde{\eta}_{4}-i v\left(\dot{\mu} \lambda_{\infty}-2 v \omega\right)\left(-\omega \tilde{\eta}_{1}-\tilde{\eta}_{3}+\beta \tilde{\eta}_{4} / 2\right)+N(U, \pi) .
\end{aligned}
$$

In this equation $\dot{\lambda}=\partial_{s}[\lambda(t(s))]$. We use the abbreviations ${ }^{18}$ (with $\left(\begin{array}{c}U \\ \bar{U}\end{array}\right)=\left(\begin{array}{l}U_{1} \\ U_{2}\end{array}\right)$ )

$N(U, \pi):=$

$\left(\begin{array}{c}-3 U_{1}^{2} \tilde{\phi}_{0}^{3} e^{i\left(\Psi_{\infty}-\Psi\right)} v^{3 / 2}-6\left|U_{1}\right|^{2} e^{i\left(\Psi-\Psi_{\infty}\right)} v^{3 / 2} \tilde{\phi}_{0}^{3}-U_{2}^{2} e^{3 i\left(\Psi-\Psi_{\infty}\right)} v^{3 / 2} \tilde{\phi}_{0}^{3}+O\left(|U|^{3}+|U|^{5}\right) \\ 3 U_{1}^{2} \tilde{\phi}_{0}^{3} e^{-i\left(\Psi_{\infty}-\Psi\right)} v^{3 / 2}+6\left|U_{1}\right|^{2} e^{-i\left(\Psi-\Psi_{\infty}\right)} v^{3 / 2} \tilde{\phi}_{0}^{3}+U_{2}^{2} e^{-3 i\left(\Psi-\Psi_{\infty}\right)} v^{3 / 2} \tilde{\phi}_{0}^{3}+O\left(|U|^{3}+|U|^{5}\right)\end{array}\right)$,

where $\tilde{\phi}_{0}(y)=\phi_{0}\left(\lambda\left(\mu_{\infty}-\mu+\lambda_{\infty}^{-1} y\right)\right)$, and

$$
\mathcal{H}(s):=\left(\begin{array}{cc}
\partial_{y}^{2}-1+3 v^{2}(s) \tilde{\phi}_{0}^{4} & 2 v^{2} \tilde{\phi}_{0}^{4} e^{2 i\left(\Psi-\Psi_{\infty}\right)} \\
-2 v^{2} \tilde{\phi}_{0}^{4} e^{-2 i\left(\Psi-\Psi_{\infty}\right)} & -\partial_{y}^{2}+1-3 v^{2}(s) \tilde{\phi}_{0}^{4}
\end{array}\right) .
$$

The orthogonality conditions $\left\langle Z, \xi_{i}\right\rangle=0, i=2, \ldots, 6$, translate to $\left\langle\left(\begin{array}{c}U \\ U\end{array}\right), \tilde{\xi}_{i}\right\rangle=0, i=$ $2, \ldots, 6$. If one differentiates this relation with respect to $s$ and uses the Leibniz rule as well as 3.5, this leads to the following system of 'ODE's', the modulation equations:

$$
\begin{aligned}
& -2 \kappa_{2} i \beta\left(\dot{\lambda} \lambda^{-1}-\beta v^{2}\right)-i \kappa_{2}\left(\dot{\beta}+\beta^{2} v^{2}\right)=-\left\langle N, \tilde{\xi}_{2}\right\rangle+\left\langle U,\left(i \partial_{s}+\mathcal{H}(s)^{*}\right) \tilde{\xi}_{2}\right\rangle, \\
& i \omega \kappa_{1}\left(\dot{\lambda} \lambda^{-1}-\beta v^{2}\right)+i \kappa_{1}\left(\dot{\omega}+\beta \omega v^{2}\right)+\frac{i}{2} \beta v\left(\dot{\mu} \lambda_{\infty}-2 \omega \nu\right) \kappa_{1} \\
& =-\left\langle N, \tilde{\xi}_{3}\right\rangle+\left\langle U,\left(i \partial_{s}+\mathcal{H}(s)^{*}\right) \tilde{\xi}_{3}\right\rangle, \\
& -i \kappa_{1} v\left(\dot{\mu} \lambda_{\infty}-2 \omega v\right)=-\left\langle N, \tilde{\xi}_{4}\right\rangle+\left\langle U,\left(i \partial_{s}+\mathcal{H}(s)^{*}\right) \tilde{\xi}_{4}\right\rangle \\
& 4 i \kappa_{2}\left(\dot{\lambda} \lambda^{-1}-\beta v^{2}\right) r=-\left\langle N, \tilde{\xi}_{5}\right\rangle+\left\langle U,\left(i \partial_{s}+\mathcal{H}(s)^{*}\right) \tilde{\xi}_{5}\right\rangle, \\
& 2 i \kappa_{2}\left(v^{2}-\dot{\gamma}+v^{2} \omega^{2}\right)+2 i \omega v \kappa_{2}\left(\dot{\mu} \lambda_{\infty}-2 v \omega\right)+\frac{i}{2} \kappa_{3}\left(\dot{\beta}+\beta^{2} v^{2}\right)+i \beta \kappa_{3}\left(\dot{\lambda} \lambda^{-1}-\beta v^{2}\right) \\
& =-\left\langle N, \tilde{\xi}_{6}\right\rangle+\left\langle U,\left(i \partial_{s}+\mathcal{H}(s)^{*}\right) \tilde{\xi}_{6}\right\rangle
\end{aligned}
$$

18 Also, recall that $\Psi_{\infty}(s, y)=\tilde{\Psi}_{\infty}(s, y)+s$. 
Of course, for all this to make sense we need to specify the 'parameters at infinity' $\left\{a_{\infty}, b_{\infty}, v_{\infty}, y_{\infty}, \gamma_{\infty}\right\}$. We shall soon see that their value is forced by the asymptotic conditions on the modulation parameters.

\subsection{Algebraic manipulations II. The equations governing the modulation parameters}

In accordance with the statement of Theorem 1.3 , we now fix the values of $(\lambda(s), \beta(s)$, $\mu(s), \omega(s), \gamma(s))\left.\right|_{s=0}=(\lambda, \beta, \mu, \omega, \gamma)$, where we require $\lambda \sim 1, \beta \sim 1$. Instead of working with the parameters $\lambda(s)$ etc., though, we shall work with $v(s)=\lambda(s) / \lambda_{\infty}(s)$, $\beta(s) v(s)-b_{\infty} / \lambda_{\infty}(s), \mu(s), \omega(s), \gamma(s)$. Start with the fourth modulation equation. Formulate it as follows:

$$
\dot{v} v^{-1}-\beta v^{2}=\left(4 i \kappa_{2}\right)^{-1}\left[-\left\langle N, \tilde{\xi}_{5}\right\rangle+\left\langle U,\left(i \partial_{s}+\mathcal{H}(s)^{*}\right) \tilde{\xi}_{5}\right\rangle\right]-b_{\infty} \lambda_{\infty}^{-1} .
$$

From the fourth and second equation, we get

$$
\dot{\beta}+\beta^{2} v^{2}=-\left(i \kappa_{2}\right)^{-1} E_{2}-\frac{\beta}{2 i \kappa_{2}} E_{5},
$$

where we use the notation $E_{j}:=-\left\langle N, \tilde{\xi}_{j}\right\rangle+\left\langle U,\left(i \partial_{s}+\mathcal{H}^{*}(s)\right) \tilde{\xi}_{j}\right\rangle$. Noting the simple identity $\left(b_{\infty} \lambda_{\infty}^{-1}\right)_{s}+\left(b_{\infty} \lambda_{\infty}^{-1}\right)^{2}=0$, we get

$$
\begin{gathered}
\dot{v}-b_{\infty} \lambda_{\infty}^{-1}(v-1)=v\left(4 i \kappa_{2}\right)^{-1} E_{5}+\beta v(v-1)^{2}+(2 v-1)\left(\beta v-b_{\infty} \lambda_{\infty}^{-1}\right), \\
\frac{d}{d s}\left(\beta v-b_{\infty} \lambda_{\infty}^{-1}\right)+\left(\beta v-b_{\infty} \lambda_{\infty}^{-1}\right) b_{\infty} \lambda_{\infty}^{-1}=-v\left(i \kappa_{2}\right)^{-1} E_{2}+\left[-\beta \frac{v}{2 i \kappa_{2}}+v \beta\left(4 i \kappa_{2}\right)^{-1}\right] E_{5} .
\end{gathered}
$$

We can further reformulate these equations as follows:

$$
\begin{aligned}
& \frac{d}{d s}\left[(v-1) \lambda_{\infty}^{-1}\right](s)=\lambda_{\infty}^{-1}\left[v\left(4 i \kappa_{2}\right)^{-1} E_{5}+\beta v(v-1)^{2}+(2 v-1)\left(\beta v-b_{\infty} \lambda_{\infty}^{-1}\right)\right], \\
& \frac{d}{d s}\left(\left[\beta v-b_{\infty} \lambda_{\infty}^{-1}\right] \lambda_{\infty}\right)=\lambda_{\infty}\left[-v\left(i \kappa_{2}\right)^{-1} E_{2}-\frac{\beta v}{4 i \kappa_{2}} E_{5}\right](s) .
\end{aligned}
$$

The condition that $v(s) \rightarrow 1$ as $s \rightarrow+\infty$, as well as the condition $\beta v(s)-b_{\infty} \lambda_{\infty}^{-1}(s)$ $\rightarrow 0$ imply the following identities:

$$
\begin{aligned}
0= & \left(\beta v-b_{\infty} \lambda_{\infty}^{-1}\right) \lambda_{\infty}(0)+\int_{0}^{\infty} \lambda_{\infty}(s)\left[-v\left(i \kappa_{2}\right)^{-1} E_{2}-\frac{\beta v}{4 i \kappa_{2}} E_{5}\right](s) d s, \\
0= & (v-1) \lambda_{\infty}^{-1}(0) \\
& +\int_{0}^{\infty} \lambda_{\infty}(s)^{-1}\left[v\left(4 i \kappa_{2}\right)^{-1} E_{5}+\beta v(v-1)^{2}+(2 v-1)\left(\beta v-b_{\infty} \lambda_{\infty}^{-1}\right)\right](s) d s,
\end{aligned}
$$

whence

$$
\begin{aligned}
0= & \left(\beta(0) \lambda(0)-b_{\infty}\right)+\int_{0}^{\infty} \lambda_{\infty}(s)\left[-v\left(i \kappa_{2}\right)^{-1} E_{2}+\frac{3 \beta v}{4 i \kappa_{2}} E_{5}\right](s) d s \\
0= & \lambda(0)-a_{\infty} \\
& +a_{\infty}^{2} \int_{0}^{\infty} \lambda_{\infty}(s)^{-1}\left[v\left(4 i \kappa_{2}\right)^{-1} E_{5}+\beta v(v-1)^{2}+(2 v-1)\left(\beta v-b_{\infty} \lambda_{\infty}^{-1}\right)\right](s) d s .
\end{aligned}
$$


Assuming the integral expressions known, this allows for solving for the coefficients $a_{\infty}, b_{\infty}$, using the Implicit Function Theorem. Moreover, we get the formulae

$$
\begin{aligned}
& v(s)-1= \\
& -\lambda_{\infty}(s) \int_{s}^{\infty} \lambda_{\infty}(\sigma)^{-1}\left[v\left(4 i \kappa_{2}\right)^{-1} E_{5}+\beta v(v-1)^{2}+(2 v-1)\left(\beta v-b_{\infty} \lambda_{\infty}^{-1}\right)\right](\sigma) d \sigma, \\
& \left(\beta v-b_{\infty} \lambda_{\infty}^{-1}\right)(s)=-\lambda_{\infty}(s)^{-1} \int_{s}^{\infty} \lambda_{\infty}(\sigma)\left[-v\left(i \kappa_{2}\right)^{-1} E_{2}-\frac{\beta v}{4 i \kappa_{2}} E_{5}\right](\sigma) d \sigma .
\end{aligned}
$$

Next, from the second, third and fourth modulation equations we gather

$$
\dot{\omega}+\beta v^{2} \omega=\left(i \kappa_{1}\right)^{-1} E_{3}-\omega\left(4 i \kappa_{2}\right)^{-1} E_{5}+\frac{\beta}{2 i \kappa_{1}} E_{4} .
$$

Introduce the quantity

$$
B(s)=\exp \left(\int_{0}^{s}\left[\beta v^{2}+\frac{1}{4 i \kappa_{2}} E_{5}\right](\sigma) d \sigma\right) .
$$

We can then write

$$
\omega(s)=B(s)^{-1} \omega(0)+\int_{0}^{s} \frac{B(\sigma)}{B(s)}\left[\left(i \kappa_{1}\right)^{-1} E_{3}+\frac{\beta}{2 i \kappa_{1}} E_{4}\right](\sigma) d \sigma .
$$

Decompose

$$
\beta v^{2}=\left(\beta v-b_{\infty} \lambda_{\infty}^{-1}\right) v+(v-1) b_{\infty} \lambda_{\infty}^{-1}+b_{\infty} \lambda_{\infty}^{-1}
$$

The stipulations $\lim _{s \rightarrow+\infty} v(s)=1$ and $\lim _{s \rightarrow+\infty} \beta v-b_{\infty} \lambda_{\infty}^{-1}=0$ then yield 19

$$
B^{-1}(s)=c \lambda_{\infty}^{-1}(s)+o(1 / s) .
$$

We then reformulate (3.9) as follows:

$$
\begin{aligned}
\omega(s)= & c \lambda_{\infty}(s)^{-1}\left[\omega(0)+\int_{0}^{\infty} B(\sigma)\left[\left(i \kappa_{1}\right)^{-1} E_{3}+\frac{\beta}{2 i \kappa_{1}} E_{4}\right](\sigma) d \sigma\right] \\
& -c \lambda_{\infty}(s)^{-1} \int_{s}^{\infty} B(\sigma)\left[\left(i \kappa_{1}\right)^{-1} E_{3}+\frac{\beta}{2 i \kappa_{1}} E_{4}\right](\sigma) d \sigma+o\left(s^{-1}\right)
\end{aligned}
$$

from which we obtain for suitable $c_{\infty}$ the asymptotic relation $\omega(s)=c_{\infty} \lambda_{\infty}^{-1}+o\left(s^{-1}\right)$, provided we can control all the integrals. From the third modulation equation we obtain

$$
\mu(s)=\mu(0)+\int_{0}^{s} \lambda_{\infty}(\sigma)^{-1}\left[2 \omega \nu-\left(i \kappa_{1} v(\sigma)\right)^{-1} E_{4}(\sigma)\right] d \sigma .
$$

If we feed in the relation 3.10 , we infer the existence of parameters $v_{\infty}, y_{\infty}$ with the property

$$
\mu(s)=\frac{2 v_{\infty} s+y_{\infty}}{a_{\infty}+b_{\infty} s}+o\left(s^{-1}\right)
$$

19 We shall soon specify the precise decay rates. 
Finally, the fifth modulation equation gives

$$
\begin{aligned}
\gamma(s)=\gamma(0)+\int_{0}^{s}\left[\nu^{2}(\sigma)-\right. & \left(2 i \kappa_{2}\right)^{-1} E_{6}(\sigma)+v^{2}(\sigma) \omega^{2}(\sigma) \\
& \left.-\frac{1}{i \kappa_{1}} \omega(\sigma) E_{4}(\sigma)-\frac{1}{2 \kappa_{2}}\left(i \kappa_{2}\right)^{-1} E_{2}(\sigma)\right] d \sigma .
\end{aligned}
$$

Last but not least, we choose $\gamma_{\infty}$ such that $\left(\Psi-\Psi_{\infty}\right)_{1}(0)=0$, where $\Psi_{\infty}(s, y)=$ $\tilde{\Psi}_{\infty}(s, y)+s$, and we define $\left(\Psi-\Psi_{\infty}\right)_{1}(s)$ to be that part of $\Psi-\Psi_{\infty}$ which only depends on $s$ (see the ensuing subsection).

\subsection{Estimates for the modulation parameters}

We now state the precise estimates for the modulation parameters which we shall work with: first, choose small positive numbers $\delta_{i}, i=1,2,3$, and $\delta>0$ with the property $\delta \ll \delta_{2} \ll \delta_{3} \ll \delta_{1}$. These shall be fixed throughout. The number $\delta$ will control the size ${ }^{20}$ of the radiation part as well as modulation parameters, while the parameters $\delta_{i}$ measure parameters in certain norms. Then we need, for a sufficiently large ${ }^{21} N=N\left(\delta_{2}, \delta_{3}, \delta_{1}\right)$ and very large $M>M\left(\delta_{2}\right)$ held fixed throughout:

$$
\begin{aligned}
& |v(s)-1| \lesssim \delta^{2}\langle s\rangle^{-1 / 2+\delta_{1}}, \\
& \sup _{1 \leq i \leq[N / 3]}\left\|\langle s\rangle^{3 / 2-2 \delta_{1}} d^{i} / d s^{i} v(s)\right\|_{L^{M}} \lesssim \delta^{2}, \\
& \left|\beta(s) v(s)-b_{\infty} \lambda_{\infty}^{-1}(s)\right| \lesssim\langle s\rangle^{-3 / 2+\delta_{1}} \delta^{2}, \\
& \sup _{1 \leq i \leq[N / 3]}\left\|\langle s\rangle^{2-2 \delta_{1}} \frac{d^{i}}{d s^{i}}\left[\beta(s) v(s)-b_{\infty} \lambda_{\infty}^{-1}(s)\right]\right\|_{L^{M}} \lesssim \delta^{2}, \\
& \left|\omega(s)-c_{\infty} \lambda_{\infty}^{-1}(s)\right| \lesssim \delta^{2}\langle s\rangle^{-3 / 2+\delta_{1}}, \\
& \sup _{1 \leq i \leq[N / 3]}\left\|\langle s\rangle^{2-2 \delta_{1}} \frac{d^{i}}{d s^{i}}\left[\omega(s)-c_{\infty} \lambda_{\infty}^{-1}(s)\right]\right\|_{L^{M}} \lesssim \delta^{2}, \\
& \left|\partial_{s}(\gamma(s)-s)-c_{\infty}^{2} \lambda_{\infty}^{-2}(s)\right| \lesssim \delta^{2}\langle s\rangle^{-1 / 2+\delta_{1}}, \\
& \sup _{1 \leq i \leq[N / 3]}\left\|\langle s\rangle^{3 / 2-2 \delta_{1}} \frac{d^{i}}{d s^{i}}\left(\frac{d}{d s}(\gamma(s)-s)-c_{\infty}^{2} \lambda_{\infty}^{-2}(s)\right)\right\|_{L^{M}} \lesssim \delta^{2}, \\
& \left|\mu(s)-\frac{2 v_{\infty} s+y_{\infty}}{a_{\infty}+b_{\infty} s}\right| \lesssim \delta^{2}\langle s\rangle^{-3 / 2+\delta_{1}}, \\
& \sup _{1 \leq i \leq[N / 3]}\left\|\langle s\rangle^{5 / 2-\delta_{1}} \frac{d^{i}}{d s^{i}}\left[\mu(s)-\frac{2 v_{\infty} s+y_{\infty}}{a_{\infty}+b_{\infty} s}\right]\right\|_{L^{M}} \lesssim \delta^{2} .
\end{aligned}
$$

\footnotetext{
20 With respect to suitable norms.

21 We shall need $N \ll N_{1}\left(\delta_{2}, \delta_{1}\right)$ and $N>N_{2}\left(\delta_{3}\right)$. The parameter $\delta_{2}$ will appear in the estimates (3.30), where we specify $N_{1}\left(\delta_{2}, \delta_{1}\right) \sim\left|\log \left(\delta_{1} / \delta_{2}\right)\right|$. The bound $N>N_{2}\left(\delta_{3}\right)$ is needed in bootstrapping the strong local dispersive estimate.
} 
The fact that we work with $L^{M}$ instead of $L^{\infty}$ for the derivatives is a technical complication due to the fact that we need a compactness property for the fixed point theorem to apply (see below).

\subsection{Algebraic manipulations II. Analysis of the radiation part}

We now look at $\left(\begin{array}{c}U \\ U\end{array}\right)$. As mentioned in the first section, we essentially break this into its root and dispersive part; more precisely, we first tweak this function a bit, after a careful analysis of the phase $\left(\Psi-\Psi_{\infty}\right)(s, y)=\left(\Psi-\tilde{\Psi}_{\infty}\right)(s, y)-s$.

From (3.4) we infer the relation 22

$\Psi-\Psi_{\infty}(s, y)$

$$
\begin{aligned}
= & \gamma(s)-s+y\left[\omega(s) v(s)-\frac{\beta(s)}{2} v(s) \lambda(s)\left(\mu_{\infty}-\mu\right)-\frac{a_{\infty} v_{\infty}-b_{\infty} y_{\infty} / 2}{a_{\infty}+b_{\infty} s}\right] \\
& +\omega(s) \lambda(s)\left(\mu_{\infty}-\mu\right)(s)+\frac{b_{\infty} y^{2}}{4\left(a_{\infty}+b_{\infty} s\right)}-\frac{\beta}{4}[v y]^{2}-\frac{\beta}{4}\left[\lambda\left[\mu_{\infty}-\mu\right]\right]^{2} \\
& -\left[\frac{v_{\infty}^{2} s a_{\infty}}{a_{\infty}+b_{\infty} s}-\frac{b_{\infty} v_{\infty} s y_{\infty}}{a_{\infty}+b_{\infty} s}+\gamma_{\infty}-\frac{b_{\infty} y_{\infty}^{2}}{4\left(a_{\infty}+s b_{\infty}\right)}\right] .
\end{aligned}
$$

Here we have also used the fact that

$$
\begin{aligned}
\Psi(s, y) & =\Psi\left(t(s), \mu_{\infty}+\lambda_{\infty}^{-1} y\right) \\
& \left.=\gamma(s)+\omega(s) \lambda(s)\left[\mu_{\infty}+\lambda_{\infty}^{-1} y\right)\right]-\frac{\beta}{4}\left(\lambda(s)\left[\mu_{\infty}+\lambda_{\infty}^{-1} y\right]-\mu(s)\right)^{2}
\end{aligned}
$$

We decompose the phase $\Psi-\Psi_{\infty}$ into two parts, $\left(\Psi-\Psi_{\infty}\right)(s, y)=\left(\Psi-\Psi_{\infty}\right)_{1}(s)+$ $\left(\Psi-\Psi_{\infty}\right)_{2}(s, y)$, where

$$
\begin{aligned}
\left(\Psi-\Psi_{\infty}\right)_{1}(s)= & \gamma(s)-s+\omega(s) \lambda(s)\left(\mu_{\infty}-\mu\right)(s)-\frac{\beta}{4}\left[\lambda\left[\mu_{\infty}-\mu\right]\right]^{2} \\
& -\left[\frac{v_{\infty}^{2} s a_{\infty}}{a_{\infty}+b_{\infty} s}-\frac{b_{\infty} v_{\infty} s y_{\infty}}{a_{\infty}+b_{\infty} s}+\gamma_{\infty}-\frac{b_{\infty} y_{\infty}^{2}}{4\left(a_{\infty}+s b_{\infty}\right)}\right],
\end{aligned}
$$

i.e., this is the part of $\Psi-\Psi_{\infty}$ which only depends on $s$ and not on $y$. Then we define

$$
\left(\begin{array}{c}
\tilde{U}(s, y) \\
\overline{\tilde{U}}(s, y)
\end{array}\right):=\left(\begin{array}{l}
e^{-i\left(\Psi-\Psi_{\infty}\right)_{1}(s)} U\left(s, y+\lambda_{\infty}\left(\mu-\mu_{\infty}\right)(s)\right) \\
e^{+i\left(\Psi-\Psi_{\infty}\right)_{1}(s)} \bar{U}\left(s, y+\lambda_{\infty}\left(\mu-\mu_{\infty}\right)(s)\right)
\end{array}\right) .
$$

The virtue of this vector-valued function is that it satisfies an equation whose linear Schrödinger operator is essentially time independent. This is also why we apply a spectral decomposition to it, instead of the seemingly more natural $\left(\begin{array}{c}U \\ \bar{U}\end{array}\right)$. Thus we decompose

$$
\left(\begin{array}{c}
\tilde{U}(s, y) \\
\overline{\tilde{U}}(s, y)
\end{array}\right)=\sum_{i=1}^{6} \lambda_{i} \eta_{i, \text { proper }}+\left(\begin{array}{c}
\tilde{U}(s, y) \\
\overline{\tilde{U}}(s, y)
\end{array}\right)_{\mathrm{dis}} .
$$

\footnotetext{
22 Recall that we also defined $\mu_{\infty}(s)=\left(2 v_{\infty} s+y_{\infty}\right) /\left(a_{\infty}+b_{\infty} s\right)$.
} 
We can then infer the parameters $\lambda_{i}, i=1, \ldots, 5$, from the orthogonality condition (1.9), while the parameter $\lambda_{6}$ is governed by a suitable ODE. We now carefully analyze these equations. First, for $j=2, \ldots, 6$, we have explicitly (recall (2.1) as well as (1.6)

$$
\left\langle\left(\begin{array}{c}
e^{i\left(\Psi_{\infty}-\Psi\right)(t)} U\left(t, y+\lambda_{\infty}\left(\mu-\mu_{\infty}\right)(t)\right) \\
e^{-i\left(\Psi_{\infty}-\Psi\right)(t)} \bar{U}\left(t, y+\lambda_{\infty}\left(\mu-\mu_{\infty}\right)(t)\right)
\end{array}\right), \xi_{j, \operatorname{proper}}(v(t) y)\right\rangle=0 .
$$

This may be recast as 23

$$
\begin{aligned}
\left\langle\left(\begin{array}{c}
\tilde{U} \\
\tilde{\tilde{U}}
\end{array}\right), \xi_{j, \text { proper }}(v(t) y)\right\rangle=\left\langle\left(\begin{array}{c}
e^{i\left(\Psi_{\infty}-\Psi\right)_{1}(t)} U\left(t, y+\lambda_{\infty}\left(\mu-\mu_{\infty}\right)(t)\right) \\
e^{-i\left(\Psi_{\infty}-\Psi\right)_{1}(t)} \bar{U}\left(t, y+\lambda_{\infty}\left(\mu-\mu_{\infty}\right)(t)\right)
\end{array}\right), \xi_{j, \operatorname{proper}}(v(t) y)\right\rangle \\
0 \\
=\left\langle\left(\begin{array}{cc}
e^{i\left(\Psi_{\infty}-\Psi\right)_{1}(t)}\left(1-e^{i\left(\Psi_{\infty}-\Psi\right)_{2}(t)}\right) & e^{-i\left(\Psi_{\infty}-\Psi\right)_{1}(t)}\left(1-e^{-i\left(\Psi_{\infty}-\Psi\right)_{2}(t)}\right)
\end{array}\right)\right. \\
\left.\left.0 \quad \begin{array}{c}
U\left(t, y+\lambda_{\infty}\left(\mu-\mu_{\infty}\right)(t)\right) \\
\bar{U}\left(t, y+\lambda_{\infty}\left(\mu-\mu_{\infty}\right)(t)\right)
\end{array}\right), \xi_{j, \operatorname{proper}}(v(t) y)\right\rangle .
\end{aligned}
$$

The point of this formulation is that as can be seen from the estimates (3.14), the quantity $\left(\Psi-\Psi_{\infty}\right)_{2}(s, y)$, when localized in $y$, decays quite rapidly in $s$. Our first task is filtering out the $\lambda_{i}, i=1, \ldots, 5$, from the above relation, while avoiding $\lambda_{6}$ if possible. From the above we have

$$
\begin{aligned}
\sum_{i=1}^{5} \lambda_{i}\left\langle\eta_{i, \text { proper }},\right. & \left.\xi_{\ell, \text { proper }}(v(t) y)\right\rangle+\lambda_{6}\left\langle\eta_{6, \text { proper }}, \xi_{\ell, \text { proper }}(v(t) y)\right\rangle+\left\langle\left(\begin{array}{c}
\tilde{U} \\
\tilde{\tilde{U}}
\end{array}\right)_{\mathrm{dis}}, \xi_{\ell, \text { proper }}(v(t) y)\right\rangle \\
0 & \left\langle\left(\begin{array}{cc}
e^{i\left(\Psi_{\infty}-\Psi\right)_{1}(t)}\left(1-e^{i\left(\Psi_{\infty}-\Psi\right)_{2}(t)}\right) & e^{-i\left(\Psi_{\infty}-\Psi\right)_{1}(t)}\left(1-e^{-i\left(\Psi_{\infty}-\Psi\right)_{2}(t)}\right)
\end{array}\right)\right. \\
0 & \left.\times\left(\begin{array}{c}
U\left(t, y+\lambda_{\infty}\left(\mu-\mu_{\infty}\right)(t)\right) \\
\bar{U}\left(t, y+\lambda_{\infty}\left(\mu-\mu_{\infty}\right)(t)\right)
\end{array}\right), \xi_{\ell, \operatorname{proper}}(v(t) y)\right\rangle
\end{aligned}
$$

where $\ell=2, \ldots, 6$. We treat these separately:

\subsection{Control over the root part of the radiation: the geometric modes $\ell=2,3,4,5$}

We commence with the case

$(\alpha) \ell=2$. Observe that

$$
\left\langle\eta_{6, \text { proper }}, \xi_{2, \text { proper }}(v(t) y)\right\rangle=0=\left\langle\eta_{2, \text { proper }}, \xi_{2, \operatorname{proper}}(v(t) y)\right\rangle
$$

Hence

$$
\begin{aligned}
\sum_{i=1}^{5} \lambda_{i}\left\langle\eta_{i, \text { proper }}, \xi_{2, \text { proper }}(v(t) y)\right\rangle+\lambda_{6}\left\langle\eta_{6, \text { proper }},\right. & \left.\xi_{2, \text { proper }}(v(t) y)\right\rangle \\
& =\sum_{i \neq 2,6} \lambda_{i}\left\langle\eta_{i, \text { proper }}, \xi_{2, \operatorname{proper}}(v(t) y)\right\rangle
\end{aligned}
$$

23 We also use the notation $\left(\Psi_{\infty}-\Psi\right)_{1}(s):=-\left(\Psi-\Psi_{\infty}\right)_{1}(s)$. 
( $\beta) \ell=3$. Next, we observe that

$$
\left\langle\eta_{6, \text { proper }}, \xi_{3, \text { proper }}(v(t) y)\right\rangle=0=\left\langle\eta_{2, \text { proper }}, \xi_{3, \text { proper }}(v(t) y)\right\rangle .
$$

Thus we have

$$
\begin{aligned}
\sum_{i=1}^{5} \lambda_{i}\left\langle\eta_{i, \text { proper }}, \xi_{3, \text { proper }}(v(t) y)\right\rangle+\lambda_{6}\left\langle\eta_{6, \text { proper }},\right. & \left.\xi_{3, \text { proper }}(v(t) y)\right\rangle \\
& =\sum_{i \neq 2,6} \lambda_{i}\left\langle\eta_{i, \text { proper }}, \xi_{3, \text { proper }}(v(t) y)\right\rangle
\end{aligned}
$$

$(\gamma) \ell=4$. Further, observe that for reasons of parity, we have

$$
\left\langle\eta_{6, \text { proper }}, \xi_{4, \text { proper }}(v(t) y)\right\rangle=0=\left\langle\eta_{2, \text { proper }}, \xi_{4, \operatorname{proper}}(v(t) y)\right\rangle .
$$

The conclusion is that

$$
\begin{aligned}
\sum_{i=1}^{5} \lambda_{i}\left\langle\eta_{i, \text { proper }}, \xi_{4, \text { proper }}(v(t) y)\right\rangle+\lambda_{6}\left\langle\eta_{6, \text { proper }}, \xi_{4, \text { proper }}(v(t) y)\right\rangle & \\
& =\sum_{i \neq 2,6} \lambda_{i}\left\langle\eta_{i, \text { proper }}, \xi_{4, \text { proper }}(v(t) y)\right\rangle
\end{aligned}
$$

( $\delta) \ell=6$. This is similar to the case $\ell=4$.

$(\epsilon) \ell=5$. Next we consider the inner product with $\xi_{5 \text {, proper }}(v(t) y)$. We note the following inner product relations:

$$
\begin{array}{ll}
\left\langle\eta_{1, \text { proper }}, \xi_{5, \text { proper }}(\nu(t) y)\right\rangle=0, & \left\langle\eta_{2, \text { proper }}, \xi_{5, \text { proper }}(v(t) y)\right\rangle=a(t), \\
\left\langle\eta_{3, \text { proper }}, \xi_{5, \text { proper }}(v(t) y)\right\rangle=0, & \left\langle\eta_{4, \text { proper }}, \xi_{5, \text { proper }}(v(t) y)\right\rangle=0, \\
\left\langle\eta_{5, \text { proper }}, \xi_{5, \text { proper }}(v(t) y)\right\rangle=0, & \left\langle\eta_{6, \text { proper }}, \xi_{5, \text { proper }}(v(t) y)\right\rangle=b(t) .
\end{array}
$$

In the immediately preceding, the function $a(t)$ can be forced to vanish nowhere upon choosing $\delta$ small enough. Moreover, we have $a(t)=C(v(t))$ for some smooth bounded function $C(\cdot)$, provided $v$ is as in (3.14). The latter observation also applies to $b(t)$. We can now infer the following relations: first

$$
\lambda_{2}(t)=(v(t)-1)\left\langle\left(\begin{array}{c}
\tilde{U} \\
\overline{\tilde{U}}
\end{array}\right)_{\mathrm{dis}}, \phi(t, y)\right\rangle+\sum_{i \neq 2} a_{2 i}(t) \lambda_{i}(t),
$$

where $\phi(t, y)$ denotes a certain time dependent vector-valued Schwartz function with respect to $y$ (with uniform decay estimates for all its derivatives, including the time derivatives), while the $a_{2 i}(t), i \neq 2,6$, decay at the same rate as $\left.\tilde{\phi}(t, y)\left(\Psi-\Psi_{\infty}\right)_{2}(t, x)\right)$, for another Schwartz function $\tilde{\phi}(t, y)$ with respect to $y$; furthermore, Lemma 5.2 below reveals that they are of size $\sim \delta^{2}$, provided the assumptions (3.14) are in force. Moreover, the functions $\phi(t, y), \tilde{\phi}(t, y)$ are of the form $C\left(v,\left(\Psi-\Psi_{\infty}\right)_{1},\left(\Psi-\Psi_{\infty}\right)_{2}, y\right)$, which is of Schwartz class with respect to $y$, and depends smoothly on the first three arguments. 
We shall henceforth denote such functions in the form $\phi(t, y)$, it being understood that this may denote different functions on different lines. We also know that the functions $a_{2 i}(t)$ depend smoothly on the functions $v,\left(\Psi-\Psi_{\infty}\right)_{1},\left(\Psi-\Psi_{\infty}\right)_{2}$.

In the same vein as 3.17, the preceding calculations allow us to infer that the coefficients $\lambda_{i}(t), i \neq 2,6$, satisfy the relations

$$
\lambda_{i}(t)=(v(t)-1)\left\langle\left(\begin{array}{c}
\tilde{U} \\
\tilde{\tilde{U}}
\end{array}\right)_{\mathrm{dis}}, \phi(t)\right\rangle+\lambda_{2}(t) a_{i 2}(t)+\lambda_{6}(t) a_{i 6}(t),
$$

where the coefficients $a_{i 2}(t), a_{i 6}(t)$ satisfy the same estimates as $a_{2 i}(t)$ (with $i \neq 2,6$ ) above. Of course if we substitute (3.17) here we can get rid of the second term on the right (choosing $\delta$ small enough). We have used the fact that

$$
\left\langle\left(\begin{array}{c}
\tilde{U} \\
\tilde{U}
\end{array}\right)_{\text {dis }}, \xi_{\ell, \text { proper }}(v(t) \cdot)\right\rangle=\left\langle\left(\begin{array}{c}
\tilde{U} \\
\tilde{\tilde{U}}
\end{array}\right)_{\mathrm{dis}}, \xi_{\ell, \text { proper }}(v(t) y)-\xi_{\ell, \text { proper }}(\cdot)\right\rangle, \quad \ell \neq 1
$$

\subsection{The equation of evolution of the exotic mode; $\ell=6$}

In order to complete the control of the root part, we thus need to finally consider $\lambda_{6}(t)$, which controls the contribution of the 'exotic mode'. This we filter out by means of

$$
2 \kappa_{2} \lambda_{6}(t)=\left\langle\left(\begin{array}{c}
\tilde{U} \\
\tilde{U}
\end{array}\right), \xi_{1, \text { proper }}\right\rangle
$$

where we recall the notation introduced before Theorem 2.1. Upon differentiation, this relation implies

$$
\begin{aligned}
i 2 \kappa_{2} \dot{\lambda}_{6}(t)= & \left\langle i \partial_{t}\left(\begin{array}{c}
\tilde{U} \\
\tilde{\tilde{U}}
\end{array}\right), \xi_{1, \text { proper }}\right\rangle \\
= & \left\langle\left(\begin{array}{c}
\tilde{U} \\
\tilde{\tilde{U}}
\end{array}\right),\left(\begin{array}{cc}
\partial_{t}\left[\Psi-\Psi_{\infty}\right]_{1} & 0 \\
0 & -\partial_{t}\left[\Psi-\Psi_{\infty}\right]_{1}
\end{array}\right) \xi_{1, \text { proper }}\right\rangle \\
& +i\left\langle\partial_{t}\left[\lambda_{\infty}\left(\mu-\mu_{\infty}\right)\right]\left(\frac{\partial_{x} \tilde{U}}{\partial_{x} \tilde{U}}\right), \xi_{1, \text { proper }}\right\rangle \\
& +\left\langle\left(\frac{e^{-i\left(\Psi-\Psi_{\infty}\right)_{1}(t)} i \partial_{t} U\left(t, y+\lambda_{\infty}\left(\mu-\mu_{\infty}\right)(t)\right)}{-e^{i\left(\Psi-\Psi_{\infty}\right)_{1}(t)} i \partial_{t} U\left(t, y+\lambda_{\infty}\left(\mu-\mu_{\infty}\right)(t)\right)}\right), \xi_{1, \text { proper }}\right\rangle
\end{aligned}
$$

We now carefully analyze each of the three expressions on the right. The key is to show that no quantity morally ${ }^{24}$ of the form $(v(t)-1)^{a} \lambda_{6}(t),(v(t)-1)^{a} \lambda_{2}(t), a=1,2$, occurs, as this would sabotage any attempt at controlling $\lambda_{6}$ by means of ODE techniques, on account of the estimates 3.14. This appears to require a lot of careful bookkeeping. Start with the first expression on the right. We have

24 Observe that for example the quantity $\partial_{t}\left(\Psi-\Psi_{\infty}\right)(t, y)$ decays like $v(t)-1$. 


$$
\begin{aligned}
\left\langle\left(\begin{array}{c}
\tilde{U} \\
\tilde{\tilde{U}}
\end{array}\right),\left(\begin{array}{cc}
\partial_{t}\left[\Psi-\Psi_{\infty}\right]_{1} & 0 \\
0 & -\partial_{t}\left[\Psi-\Psi_{\infty}\right]_{1}
\end{array}\right) \xi_{1, \text { proper }}\right\rangle \\
=\sum_{i \neq 2,6} \lambda_{j}(t)\left\langle\eta_{j, \operatorname{proper}},\left(\begin{array}{cc}
\partial_{t}\left[\Psi-\Psi_{\infty}\right]_{1} & 0 \\
0 & -\partial_{t}\left[\Psi-\Psi_{\infty}\right]_{1}
\end{array}\right) \xi_{1, \text { proper }}\right\rangle \\
+\left\langle\left(\begin{array}{c}
\tilde{U} \\
\overline{\tilde{U}}
\end{array}\right)_{\mathrm{dis}},\left(\begin{array}{cc}
\partial_{t}\left[\Psi-\Psi_{\infty}\right]_{1} & 0 \\
0 & -\partial_{t}\left[\Psi-\Psi_{\infty}\right]_{1}
\end{array}\right) \xi_{1, \text { proper }}\right\rangle .
\end{aligned}
$$

Next, write

$$
\begin{aligned}
\left\langle\partial_{t}\left[\lambda_{\infty}\left(\mu-\mu_{\infty}\right)\right]\left(\begin{array}{c}
\partial_{x} \tilde{U} \\
\partial_{x} \tilde{U}
\end{array}\right), \xi_{1, \text { proper }}\right\rangle= & \partial_{t}\left[\lambda_{\infty}\left(\mu-\mu_{\infty}\right)\right] \sum_{j=1}^{6} \lambda_{j}\left\langle\partial_{x} \eta_{j, \text { proper }}, \xi_{1, \text { proper }}\right\rangle \\
& +\partial_{t}\left[\lambda_{\infty}\left(\mu-\mu_{\infty}\right)\right]\left\langle\left(\frac{\partial_{x} \tilde{U}_{\mathrm{dis}}}{\partial_{x} \tilde{U}_{\text {dis }}}\right), \xi_{1, \text { proper }}\right\rangle .
\end{aligned}
$$

Carefully observe from 3.14 that $\left|\partial_{t}\left[\lambda_{\infty}\left(\mu-\mu_{\infty}\right)\right](t)\right| \lesssim\langle t\rangle^{-3 / 2+\delta_{1}}$. Finally, consider the contribution of

$$
\left\langle\left(\frac{e^{-i\left(\Psi-\Psi_{\infty}\right)_{1}(t)} i \partial_{t} U\left(t, y+\lambda_{\infty}\left(\mu-\mu_{\infty}\right)(t)\right)}{-e^{-i\left(\Psi-\Psi_{\infty}\right)_{1}(t)} i \partial_{t} U\left(t, y+\lambda_{\infty}\left(\mu-\mu_{\infty}\right)(t)\right)}\right), \xi_{1, \text { proper }}\right\rangle
$$

The idea is to use the equation 3.5 as well as integration by parts. Observe that

$$
\begin{aligned}
\left(\begin{array}{cc}
\partial_{y}^{2}-1+3 v^{2} \phi_{0}^{4}(v(t) y) & 2 v^{2} \phi_{0}^{4}(v(t) y) \\
-2 v^{2} \phi_{0}^{4}(v(t) y) & -\partial_{y}^{2}+1-3 v^{2} \phi_{0}^{4}(v(t) y)
\end{array}\right)\left(\begin{array}{c}
e^{i\left(\Psi_{\infty}-\Psi\right)_{1}(t)} U\left(y+\lambda_{\infty}\left[\mu-\mu_{\infty}\right](t), t\right) \\
e^{-i\left(\Psi_{\infty}-\Psi\right)_{1}(t)} \bar{U}\left(y+\lambda_{\infty}\left[\mu-\mu_{\infty}\right](t), t\right)
\end{array}\right) \\
=\left(\begin{array}{cc}
e^{i\left(\Psi_{\infty}-\Psi\right)_{1}(t)} & 0 \\
0 & e^{-i\left(\Psi_{\infty}-\Psi\right)_{1}(t)}
\end{array}\right) \\
\\
\cdot\left(\begin{array}{cc}
\partial_{y}^{2}-1+3 v^{2} \phi_{0}^{4}(v(t) y) & 2 v^{2} e^{2 i\left(\Psi-\Psi_{\infty}\right)_{1}} \phi_{0}^{4}(v(t) y) \\
-2 v^{2} e^{-2 i\left(\Psi-\Psi_{\infty}\right)_{1}} \phi_{0}^{4}(v(t) y) & -\partial_{y}^{2}+1-3 v^{2} \phi_{0}^{4}(v(t) y)
\end{array}\right)\left(\begin{array}{c}
U \\
U
\end{array}\right)(\cdot) .
\end{aligned}
$$

This shows that we can reformulate 3.20 as follows:

$$
\begin{aligned}
& \left\langle\left(\begin{array}{cc}
e^{i\left(\Psi_{\infty}-\Psi\right)_{1}(t)} & 0 \\
0 & e^{-i\left(\Psi_{\infty}-\Psi\right)_{1}(t)}
\end{array}\right)\left[i \partial_{t}+\mathcal{H}_{1}(t)\right]\left(\left(\begin{array}{l}
U \\
\bar{U}
\end{array}\right)\left(y+\lambda_{\infty}\left[\mu-\mu_{\infty}\right](t), t\right)\right), \xi_{1, \text { proper }}\right\rangle \\
& -\left\langle\left(\begin{array}{c}
e^{i\left(\Psi_{\infty}-\Psi\right)_{1}(t)} U\left(y+\lambda_{\infty}\left[\mu-\mu_{\infty}\right](t), t\right) \\
e^{-i\left(\Psi_{\infty}-\Psi\right)_{1}(t)} \bar{U}\left(y+\lambda_{\infty}\left[\mu-\mu_{\infty}\right](t), t\right)
\end{array}\right), \tilde{\mathcal{H}}(t)^{*} \xi_{1, \text { proper }}\right\rangle
\end{aligned}
$$

where

$$
\begin{aligned}
\tilde{\mathcal{H}}(t)^{*} & =\left(\begin{array}{cc}
\partial_{y}^{2}-1+3 v^{2} \phi_{0}^{4}(v(t) y) & -2 v^{2} \phi_{0}^{4}(v(t) y) \\
+2 v^{2} \phi_{0}^{4}(v(t) y) & -\partial_{y}^{2}+1-3 v^{2} \phi_{0}^{4}(v(t) y)
\end{array}\right), \\
\mathcal{H}_{1}(t) & =\left(\begin{array}{cc}
\partial_{y}^{2}-1+3 v^{2} \phi_{0}^{4}(v(t) y) & 2 v^{2} e^{2 i\left(\Psi-\Psi_{\infty}\right)_{1}} \phi_{0}^{4}(v(t) y) \\
-2 v^{2} e^{-2 i\left(\Psi-\Psi_{\infty}\right)_{1}} \phi_{0}^{4}(v(t) y) & -\partial_{y}^{2}+1-3 v^{2} \phi_{0}^{4}(v(t) y)
\end{array}\right) .
\end{aligned}
$$


Moreover, we have

$$
\tilde{\mathcal{H}}(t)^{*} \xi_{1, \text { proper }}=\left(\begin{array}{cc}
3 v^{2} \phi_{0}^{4}(v(t) y)-3 \phi_{0}^{4}(y) & -2 v^{2} \phi_{0}^{4}(v(t) y)+2 \phi_{0}^{4} \\
-\left[-2 v^{2} \phi_{0}^{4}(v(t) y)+2 \phi_{0}^{4}\right] & -\left[3 v^{2} \phi_{0}^{4}(v(t) y)-3 \phi_{0}^{4}(y)\right]
\end{array}\right) \xi_{1, \text { proper }},
$$

which is of the form $\left(\begin{array}{c}\alpha \\ -\alpha\end{array}\right)$. This reveals that

$$
\begin{aligned}
\left\langle\left(\begin{array}{c}
e^{i\left(\Psi_{\infty}-\Psi\right)_{1}(t)} U\left(y+\lambda_{\infty}\left[\mu-\mu_{\infty}\right](t), t\right) \\
e^{-i\left(\Psi_{\infty}-\Psi\right)_{1}(t)} \bar{U}\left(y+\lambda_{\infty}\left[\mu-\mu_{\infty}\right](t), t\right)
\end{array}\right), \tilde{\mathcal{H}}(t)^{*} \xi_{1, \text { proper }}\right\rangle \\
=\sum_{j \neq 2,6} \lambda_{j}(t)\left\langle\eta_{j, \text { proper }}, \tilde{\mathcal{H}}(t)^{*} \xi_{1, \text { proper }}\right\rangle+\left\langle\left(\begin{array}{c}
\tilde{U} \\
\tilde{U}
\end{array}\right)_{\text {dis }}, \tilde{\mathcal{H}}(t)^{*} \xi_{1, \text { proper }}\right\rangle
\end{aligned}
$$

Also, note that $\tilde{\mathcal{H}}(t)^{*} \xi_{1 \text {, proper }}=(v(t)-1) \phi(t, \cdot)$ for a suitable (vector-valued) Schwartz function $\phi(t, \cdot)$. We now need to carefully analyze the expression

$\left\langle\left(\begin{array}{cc}e^{i\left(\Psi_{\infty}-\Psi\right)_{1}(t)} & 0 \\ 0 & e^{-i\left(\Psi_{\infty}-\Psi\right)_{1}(t)}\end{array}\right)\left(\left[i \partial_{t}+\mathcal{H}_{1}(t)\right]\left(\begin{array}{c}U \\ \bar{U}\end{array}\right)\right)\left(y+\lambda_{\infty}\left[\mu-\mu_{\infty}\right](t), t\right), \xi_{1, \text { proper }}\right\rangle$.

We reformulate this as 25

$$
\left\langle\left[i \partial_{t}+\tilde{\mathcal{H}}_{1}(t)\right]\left(\left(\begin{array}{l}
U \\
\bar{U}
\end{array}\right)(y, t)\right),\left(\begin{array}{cc}
e^{-i\left(\Psi_{\infty}-\Psi\right)_{1}(t)} & 0 \\
0 & e^{+i\left(\Psi_{\infty}-\Psi\right)_{1}(t)}
\end{array}\right) \xi_{1, \operatorname{proper}}\left(y-\lambda_{\infty}\left[\mu-\mu_{\infty}\right](t)\right)\right\rangle
$$

and use 3.5 , in which we schematically write the right hand side as $\dot{\pi} \partial_{\pi} W+N(U, \pi)$, where the first summand refers to those expressions which only involve the modulation parameters and their derivatives, and not (explicitly) the radiation. Then we can schematically rewrite the above as

$$
\left\langle\dot{\pi} \partial_{\pi} W, \tilde{\xi}_{1, \text { proper }}\right\rangle+\left\langle N(U, \pi), \tilde{\xi}_{1, \text { proper }}\right\rangle+\left\langle\left(e^{i\left(\Psi-\Psi_{\infty}\right)_{2}(t)}-1\right)\left(\begin{array}{c}
U \\
\bar{U}
\end{array}\right), \phi(t, \cdot)\right\rangle,
$$

where we have introduced the notation

$$
\left(\begin{array}{cc}
e^{-i\left(\Psi_{\infty}-\Psi\right)_{1}(t)} & 0 \\
0 & e^{-i\left(\Psi-\Psi_{\infty}\right)_{1}(t)}
\end{array}\right) \xi_{1, \text { proper }}\left(y-\lambda_{\infty}\left(\mu-\mu_{\infty}\right)(t)\right)=\tilde{\xi}_{1, \text { proper }}
$$

We now carefully analyze the first two expressions in (3.21), again in order to check that these do not implicitly contain expressions of the form $(v(t)-1)^{a} \lambda_{2}(t),(v(t)-1)^{a} \lambda_{6}(t)$, $a=1,2$. The third expression in (3.21) turns out to be small, as we will see later on.

Now expand the schematic expression $\left\langle\dot{\pi} \partial_{\pi} W, \tilde{\xi}_{1 \text {,proper }}\right\rangle$, invoking 3.5. First one obtains

$$
\left\langle\left(\dot{\lambda} \lambda^{-1}-\beta \nu^{2}\right)\left(\tilde{\eta}_{2}-\frac{\beta}{2} \tilde{\eta}_{5}+\omega \tilde{\eta}_{4}\right), \tilde{\xi}_{1, \text { proper }}\right\rangle .
$$

We note that the vectors $\tilde{\eta}_{j}$ appearing here carry the phases $e^{ \pm i\left(\Psi-\Psi_{\infty}\right)}$. Thus by modifying them by errors of size $O\left(\left(e^{i\left(\Psi_{\infty}-\Psi\right)_{2}}-1\right) \phi(t, \cdot)\right)$, we can replace these phases by

25 Here we define $\tilde{\mathcal{H}}_{1}(s)$ like $\mathcal{H}(s)$ but with $\Psi-\Psi_{\infty}$ replaced by $\left(\Psi-\Psi_{\infty}\right)_{1}$. 
$e^{ \pm i\left(\Psi-\Psi_{\infty}\right)_{1}}$. By abuse of notation we shall refer to these vectors again as $\tilde{\eta}_{j}$. Then we potentially have $\left\langle\tilde{\eta}_{2}, \tilde{\xi}_{1 \text {,proper }}\right\rangle \neq 0$. Using the fifth modulation equation, we recall that

$$
4 i \kappa_{2}\left(\dot{\lambda} \lambda^{-1}-\beta \nu^{2}\right)=-\left\langle N(U, \pi), \tilde{\xi}_{5}\right\rangle+\left\langle\left(\begin{array}{l}
U \\
\bar{U}
\end{array}\right),\left(i \partial_{t}+\mathcal{H}(t)^{*}\right) \tilde{\xi}_{5}\right\rangle
$$

where

$$
\tilde{\xi}_{5}(t, y)=\left(\begin{array}{l}
e^{i\left(\Psi-\Psi_{\infty}\right)(t, y)} \sqrt{v}(t) \phi_{0}\left(v(t)\left[y-\lambda_{\infty}\left(\mu-\mu_{\infty}\right)(t)\right]\right) \\
e^{-i\left(\Psi-\Psi_{\infty}\right)(t)} \sqrt{v}(t) \phi_{0}\left(\nu(t)\left[y-\lambda_{\infty}\left(\mu-\mu_{\infty}\right)(t)\right]\right)
\end{array}\right) .
$$

We put $\tilde{\phi}(y):=y^{2} \phi_{0}(y)$ and calculate

$$
\begin{aligned}
i \partial_{t}\left(\begin{array}{c}
e^{i\left(\Psi-\Psi_{\infty}\right)(t, y)} \sqrt{v}(t) \tilde{\phi}\left(v(t)\left[y-\lambda_{\infty}\left(\mu-\mu_{\infty}\right)(t)\right]\right) \\
e^{-i\left(\Psi-\Psi_{\infty}\right)(t, y)} \sqrt{v}(t) \tilde{\phi}\left(v(t)\left[y-\lambda_{\infty}\left(\mu-\mu_{\infty}\right)(t)\right]\right)
\end{array}\right) \\
=i\left(\begin{array}{c}
i \partial_{t}\left(\Psi-\Psi_{\infty}\right)(t, y) e^{i\left(\Psi-\Psi_{\infty}\right)(t, y)} \sqrt{v}(t) \tilde{\phi}\left(v(t)\left[y-\lambda_{\infty}\left(\mu-\mu_{\infty}\right)(t)\right]\right) \\
-i \partial_{t}\left(\Psi-\Psi_{\infty}\right)(t, y) e^{-i\left(\Psi-\Psi_{\infty}\right)(t, y)} \sqrt{v}(t) \tilde{\phi}\left(v(t)\left[y-\lambda_{\infty}\left(\mu-\mu_{\infty}\right)(t)\right]\right)
\end{array}\right) \\
+i \sqrt{v}(t)\left[-\partial_{t}\left[\lambda(t)\left(\mu-\mu_{\infty}\right)(t)\right]+y \dot{v}(t)\right] \\
+\left(\begin{array}{c}
e^{i\left(\Psi-\Psi_{\infty}\right)(t, y)} \nabla \phi_{0}\left(v(t)\left[y-\lambda_{\infty}\left(\mu-\mu_{\infty}\right)(t)\right]\right) \\
e^{-i\left(\Psi-\Psi_{\infty}\right)(t, y)} \nabla \phi_{0}\left(v(t)\left[y-\lambda_{\infty}\left(\mu-\mu_{\infty}\right)(t)\right]\right)
\end{array}\right) \\
+\frac{i \dot{v}(t)}{2 \sqrt{v}(t)}\left(\begin{array}{c}
e^{i\left(\Psi-\Psi_{\infty}\right)(t, y)} \sqrt{v}(t) \phi_{0}\left(v(t)\left[y-\lambda_{\infty}\left(\mu-\mu_{\infty}\right)(t)\right]\right) \\
e^{-i\left(\Psi-\Psi_{\infty}\right)(t, y)} \sqrt{v}(t) \phi_{0}\left(v(t)\left[y-\lambda_{\infty}\left(\mu-\mu_{\infty}\right)(t)\right]\right)
\end{array}\right)
\end{aligned}
$$

Further note that for a certain $k \neq 1$,

$$
\begin{aligned}
& \mathcal{H}(t)^{*} \tilde{\xi}_{5} \\
& =\left(\begin{array}{cc}
\partial_{y}^{2}-1+3 v^{2} \phi_{0}^{4}\left(v(t)\left[y+\lambda_{\infty}\left(\mu_{\infty}-\mu\right)\right]\right) & -2 v^{2} \phi_{0}^{4}\left(v(t)\left[y+\lambda_{\infty}\left(\mu_{\infty}-\mu\right)\right]\right) e^{2 i\left(\Psi-\Psi_{\infty}\right)} \\
+2 v^{2} \phi_{0}^{4}\left(v(t)\left[y+\lambda_{\infty}\left(\mu_{\infty}-\mu\right)\right]\right) e^{-2 i\left(\Psi-\Psi_{\infty}\right)} & -\left[\partial_{y}^{2}-1+3 v^{2} \phi_{0}^{4}\left(v(t)\left[y+\lambda_{\infty}\left(\mu_{\infty}-\mu\right)\right]\right)\right]
\end{array}\right) \tilde{\xi}_{5} \\
& =\left(\begin{array}{cc}
v^{2}-1 & 0 \\
0 & -\left(v^{2}-1\right)
\end{array}\right) \tilde{\xi}_{5}+v^{2} \tilde{\xi}_{k}+O\left(\partial_{y}^{1,2}\left[\Psi-\Psi_{\infty}\right] \phi(t, \cdot)\right) .
\end{aligned}
$$

Then we observe that

$$
\begin{aligned}
&\left\langle\left(\begin{array}{c}
U \\
\bar{U}
\end{array}\right),\left(\begin{array}{cc}
v^{2}-1 & 0 \\
0 & -\left(v^{2}-1\right)
\end{array}\right) \tilde{\xi}_{5}+v^{2} \tilde{\xi}_{k}\right\rangle \\
&=\sum_{j \neq 2,6} \lambda_{j}\left\langle\eta_{j, \text { proper }},\left(\begin{array}{cc}
v^{2}-1 & 0 \\
0 & -\left(v^{2}-1\right)
\end{array}\right) \xi_{5, \operatorname{proper}}(v(t) y)\right\rangle \\
&+\left\langle\left(\begin{array}{c}
\tilde{U} \\
\overline{\tilde{U}}
\end{array}\right)_{\mathrm{dis}},\left(\begin{array}{cc}
v^{2}-1 & 0 \\
0 & -\left(v^{2}-1\right)
\end{array}\right) \xi_{5, \operatorname{proper}}(v(t) y)\right\rangle \\
&+\left\langle\left(e^{i\left(\Psi-\Psi_{\infty}\right)_{2}(t, \cdot)}-1\right)\left(\begin{array}{c}
U \\
\bar{U}
\end{array}\right), \phi(t, \cdot)\right\rangle
\end{aligned}
$$


Continue by observing that $\left\langle\tilde{\eta}_{5,4,1}, \tilde{\xi}_{1 \text {, proper }}\right\rangle=0$, provided we abuse notation and change the phase in $\tilde{\eta}_{i}$ to $\left(\Psi-\Psi_{\infty}\right)_{1}$, which generates errors of the type $O\left(\left(e^{i\left(\Psi-\Psi_{\infty}\right)_{2}(t)}-1\right)\right.$ $\cdot \phi(t, \cdot))$. Continuing in this fashion, we note that $\left\langle\tilde{\eta}_{3}, \tilde{\xi}_{1 \text {,proper }}\right\rangle \neq 0$ generically (but this function will only be of size $O((v(t)-1)))$. From the third modulation equation we get

$$
-i \kappa_{1} v\left(\dot{\mu} \lambda_{\infty}-2 \omega \nu\right)=-\left\langle N(U, \pi), \tilde{\xi}_{4}\right\rangle+\left\langle\left(\begin{array}{c}
U \\
\bar{U}
\end{array}\right),\left(i \partial_{s}+\mathcal{H}(s)^{*}\right) \tilde{\xi}_{4}\right\rangle
$$

Write as before

$$
\begin{aligned}
\partial_{s} \tilde{\xi}_{4}= & \left(\begin{array}{cc}
i \partial_{s}\left[\Psi-\Psi_{\infty}\right](s) & 0 \\
0 & -i \partial_{s}\left[\Psi-\Psi_{\infty}\right](s)
\end{array}\right) \tilde{\xi}_{4}+\left[\dot{v} y+\partial_{s}\left[\lambda\left(\mu_{\infty}-\mu\right)\right]\right] \partial_{x} \tilde{\xi}_{4} \\
& +O(\dot{v} \phi(t, \cdot)) .
\end{aligned}
$$

Moreover, we have (for a suitable $j \neq 1$ )

$\mathcal{H}(s)^{*} \tilde{\xi}_{4}=$

$\left(\begin{array}{cc}\partial_{y}^{2}-1+3 v^{2}(s) \phi_{0}^{4}\left(v(s)\left(y+\lambda_{\infty}\left(\mu_{\infty}-\mu\right)\right)\right) & -2 v^{2} \phi_{0}^{4}\left(v(s)\left(y+\lambda_{\infty}\left(\mu_{\infty}-\mu\right)\right)\right) e^{2 i\left(\Psi-\Psi_{\infty}\right)(s)} \\ +2 v^{2} \phi_{0}^{4}\left(v(s)\left(y+\lambda_{\infty}\left(\mu_{\infty}-\mu\right)\right)\right) e^{-2 i\left(\Psi-\Psi_{\infty}\right)(s)} & -\left(\partial_{y}^{2}-1+3 v^{2}(s) \phi_{0}^{4}\left(v(s)\left(y+\lambda_{\infty}\left(\mu_{\infty}-\mu\right)\right)\right)\right.\end{array}\right) \tilde{\xi}_{4}$ $=\left(\begin{array}{cc}v^{2}-1 & 0 \\ 0 & -\left(v^{2}-1\right)\end{array}\right) \tilde{\xi}_{4}+v^{2} \tilde{\xi}_{j}+O\left(\partial_{y}^{1,2}\left[\Psi-\Psi_{\infty}\right]_{2} \phi(t, \cdot)\right)$.

Thus we obtain

$$
\begin{aligned}
&\left\langle\mathcal{H}(s)^{*} \tilde{\xi}_{4},\left(\begin{array}{c}
U \\
\bar{U}
\end{array}\right)\right\rangle=\sum_{j \neq 2,6} \lambda_{j}\left\langle\eta_{j, \text { proper }},\left(\begin{array}{cc}
v^{2}-1 & 0 \\
0 & -\left(v^{2}-1\right)
\end{array}\right) \xi_{4, \operatorname{proper}}(v(s) y)\right\rangle \\
& \quad+\left\langle\left(\begin{array}{c}
\tilde{U} \\
\overline{\tilde{U}}
\end{array}\right)_{\mathrm{dis}},\left(\begin{array}{cc}
v^{2}-1 & 0 \\
0 & -\left(v^{2}-1\right)
\end{array}\right) \xi_{4, \operatorname{proper}}(v(s) y)\right\rangle+O\left(\partial_{y}^{1,2}\left[\Psi-\Psi_{\infty}\right]_{2} \phi(t, \cdot)\right) \\
&+ O\left(\left(e^{i\left(\Psi-\Psi_{\infty}\right)_{2}}-1\right) \phi(t, \cdot)\right) .
\end{aligned}
$$

The preceding observations allow us to control the expression $\left\langle\dot{\pi} \partial_{\pi} W, \tilde{\xi}_{1 \text {,proper }}\right\rangle$ in 3.21 . Observe that in the preceding we also generated the (schematic) terms $(v(t)-1)$ $\cdot\left\langle N(U, \pi), \tilde{\xi}_{4}\right\rangle,(v(t)-1)\left\langle N(U, \pi), \tilde{\xi}_{5}\right\rangle$. Now consider the terms at least quadratic with respect to the radiation in 3.21 , i.e., the expression $\left\langle N(U, \pi), \tilde{\xi}_{1 \text {,proper }}\right\rangle$. We are predominantly concerned with the quadratic contribution, which we spell out explicitly:

$$
\left\langle\left(\begin{array}{c}
-3 U^{2} \tilde{\phi}_{0}^{3} e^{i\left(\Psi_{\infty}-\Psi\right)} v^{3 / 2}-6|U|^{2} e^{i\left(\Psi-\Psi_{\infty}\right)} v^{3 / 2} \tilde{\phi}_{0}^{3}-\bar{U}^{2} e^{3 i\left(\Psi-\Psi_{\infty}\right)} v^{3 / 2} \tilde{\phi}_{0}^{3} \\
3 \bar{U}^{2} \tilde{\phi}_{0}^{3} e^{-i\left(\Psi_{\infty}-\Psi\right)} v^{3 / 2}+6|U|^{2} e^{-i\left(\Psi-\Psi_{\infty}\right)} v^{3 / 2} \tilde{\phi}_{0}^{3}-U^{2} e^{-3 i\left(\Psi-\Psi_{\infty}\right)} v^{3 / 2} \tilde{\phi}_{0}^{3}
\end{array}\right), \tilde{\xi}_{1, \text { proper }}\right\rangle,
$$

where we recall $\tilde{\phi}_{0}$ is $\phi_{0}$ evaluated at $v(s) y+\lambda\left(\mu_{\infty}-\mu\right)$. Now we substitute

$$
\left(\begin{array}{c}
\tilde{U} \\
\tilde{\tilde{U}}
\end{array}\right)=\sum_{i=1}^{5} \lambda_{i} \eta_{i, \text { proper }}+\lambda_{6} \eta_{6, \text { proper }}+\left(\begin{array}{c}
\tilde{U} \\
\overline{\tilde{U}}
\end{array}\right)_{\mathrm{dis}}
$$


Use (3.17) to reformulate this a: ${ }^{26}$

$$
\begin{aligned}
\left(\begin{array}{c}
\tilde{U} \\
\tilde{\tilde{U}}
\end{array}\right)(t, \cdot)= & \sum_{i \neq 2,6} \lambda_{i}(t)\left(\eta_{i, \text { proper }}+a_{2 i}(t) \eta_{2, \text { proper }}\right)+\lambda_{6}(t)\left(\eta_{6, \text { proper }}+a_{26}(t) \eta_{2, \text { proper }}\right) \\
& +\left(\begin{array}{c}
\tilde{U} \\
\tilde{U}
\end{array}\right)_{\mathrm{dis}}(t, \cdot)+(v(t)-1) \phi(t, \cdot)\left\langle\left(\begin{array}{c}
\tilde{U} \\
\tilde{\tilde{U}}
\end{array}\right)_{\mathrm{dis}}(t, \cdot), \phi(t, \cdot)\right\rangle .
\end{aligned}
$$

Back to 3.22, we first rewrite

$$
\begin{aligned}
\left\langle\left(\begin{array}{rl}
-U^{2} \tilde{\phi}_{0}^{3} e^{i\left(\Psi_{\infty}-\Psi\right)(t, \cdot)} v^{3 / 2} \\
\bar{U}^{2} \tilde{\phi}_{0}^{3} e^{-i\left(\Psi_{\infty}-\Psi\right)(t, \cdot)} v^{3 / 2}
\end{array}\right), \tilde{\xi}_{1, \text { proper }}\right\rangle= & \left\langle\left(\begin{array}{c}
-\tilde{U}^{2} \phi_{0}^{3}(v \cdot) v^{3 / 2} \\
\overline{\tilde{U}}^{2} \phi_{0}^{3}\left(v \cdot v^{3 / 2}\right.
\end{array}\right), \xi_{1, \text { proper }}\right\rangle \\
& +O\left(\left(e^{i\left(\Psi-\Psi_{\infty}\right)_{2}(t, \cdot)}-1\right) \phi(t, \cdot)\right) .
\end{aligned}
$$

Then note that schematically

$$
\begin{aligned}
& \frac{1}{2}\left\langle\left(\begin{array}{c}
-\tilde{U}^{2} \phi_{0}^{3}(v \cdot) v^{3 / 2} \\
\tilde{U}^{2} \phi_{0}^{3}(v \cdot) v^{3 / 2}
\end{array}\right), \xi_{1, \text { proper }}\right\rangle=v^{3 / 2} \Im\left\langle\tilde{U}_{\mathrm{dis}}^{2} \phi_{0}^{3}(v \cdot), \phi_{0}\right\rangle \\
& \quad+2 \lambda_{6}\left[\sum_{i \neq 2,6} \lambda_{i} \nu^{3 / 2} \Im\left\langle\eta_{i, \text { proper }}^{1}+a_{2 i} \eta_{2, \text { proper }}, \phi_{0}^{4}(\nu \cdot)\left(\eta_{6, \text { proper }}+a_{26}(t) \eta_{2, \text { proper }}\right)^{1}\right\rangle\right. \\
& \left.\quad+\Im\left\langle\phi(t, \cdot), \tilde{U}_{\mathrm{dis}}\right\rangle\right]+\sum_{i, j} a_{i j}(t) \lambda_{i} \lambda_{j}(t)+\sum_{j=1,2}(v(t)-1)^{j}\left\langle\tilde{U}_{\mathrm{dis}}, \phi_{1}(t, \cdot)\right\rangle\left\langle\tilde{U}_{\mathrm{dis}}, \phi_{2}(t, \cdot)\right\rangle .
\end{aligned}
$$

The expression

$$
\left\langle\left(\begin{array}{c}
-\overline{\tilde{U}}^{2} e^{3 i\left(\Psi-\Psi_{\infty}\right)} \phi_{0}^{3}(v(t) y) v^{3 / 2} \\
\tilde{U}^{2} e^{-3 i\left(\Psi-\Psi_{\infty}\right)} \phi_{0}^{3}(v(t) y) v^{3 / 2}
\end{array}\right), \tilde{\xi}_{1, \text { proper }}\right\rangle
$$

is handled similarly. Moreover, it is easily seen that

$$
\left\langle\left(\begin{array}{c}
-|\tilde{U}|^{2} v^{3 / 2} \phi_{0}^{3}(v y) \\
|\tilde{U}|^{2} v^{3 / 2} \phi_{0}^{3}(v y)
\end{array}\right), \xi_{1, \text { proper }}\right\rangle=0 .
$$

Finally, we can summarize the discussion following (3.19) in the following schematic equality 27

$$
\begin{aligned}
& \dot{\lambda}_{6}=\lambda_{6}\left[\left\langle\left(\begin{array}{c}
\tilde{U} \\
\tilde{U}
\end{array}\right)_{\mathrm{dis}}, \phi\right\rangle+(v-1)\left\langle\left(\begin{array}{c}
\tilde{U} \\
\tilde{U}
\end{array}\right)_{\mathrm{dis}}, \phi\right\rangle+\dot{v}+\lambda_{6}(v-1)+\left\langle\phi\left(e^{i\left(\Psi-\Psi_{\infty}\right)_{2}}-1\right), \psi\right\rangle\right] \\
& +\left\langle N\left(\tilde{U}_{\mathrm{dis}}^{2}, \pi\right), \phi_{1}\right\rangle+(v-1)\left\langle N\left(\tilde{U}_{\mathrm{dis}}^{2}, \pi\right), \phi_{2}\right\rangle+\sum_{a=1,2}(v-1)^{a}\left\langle\left(\begin{array}{c}
\tilde{U} \\
\tilde{\tilde{U}}
\end{array}\right)_{\mathrm{dis}}, \phi\right\rangle+\dot{v}\left\langle\left(\begin{array}{c}
\tilde{U} \\
\tilde{U}
\end{array}\right)_{\mathrm{dis}}, \phi\right\rangle \\
& +(\nu-1)\left\langle\left(\begin{array}{c}
\tilde{U} \\
\tilde{U}
\end{array}\right)_{\mathrm{dis}}, \phi_{1}\right\rangle\left\langle\left(\begin{array}{c}
\tilde{U} \\
\tilde{U}
\end{array}\right)_{\mathrm{dis}}, \phi_{2}\right\rangle+O\left(\left\langle U^{3}+U^{5}+\left[(v-1)^{2}+\dot{v}\right] U^{2}, \phi\right\rangle\right) .
\end{aligned}
$$

26 As usual, $\phi(t, \cdot)$ represents various Schwartz functions, which in addition to all their derivatives, both with respect to $t$ and $x$, satisfy uniform decay estimates. Also, $\partial_{t} \phi$ is of size at most $\dot{v}$. 27 The first instance of $\left\langle N\left(\tilde{U}_{\text {dis }}^{2}, \pi\right), \phi_{1}\right\rangle$ refers to the symplectic form $\left\langle\tilde{U}_{\text {dis }}^{2}-\overline{\tilde{U}}_{\text {dis }}^{2}, \phi_{1}\right\rangle$. 
As usual, the functions $\phi, \psi$ etc. represent Schwartz functions with respect to the spatial variable and uniformly so with respect to time. They can be written in the form $C\left(\nu,\left(\Psi-\Psi_{\infty}\right)_{1},\left(\Psi-\Psi_{\infty}\right)_{2}, y\right)$. One easily checks that all these functions have time derivatives decaying like $\dot{v}$. In the arguments below, we shall omit the time dependence, as one easily checks that any additional terms generated by this additional time dependence of the $\phi$ etc. (for example when performing integrations by parts in $t$ ) can be handled by exactly the same methods or are much simpler to estimate. We now impose the condition $\lim _{s \rightarrow+\infty} \lambda_{6}(s)=0$. If we introduce the integrating factor

$$
\begin{aligned}
\Lambda(t)= & \int_{t}^{\infty}\left[\left\langle\left(\begin{array}{c}
\tilde{U}(s, \cdot) \\
\tilde{\tilde{U}}(s, \cdot)
\end{array}\right)_{\mathrm{dis}}, \phi\right\rangle+(v-1)(s)\left\langle\left(\begin{array}{c}
\tilde{U}(s, \cdot) \\
\tilde{\tilde{U}}(s, \cdot)
\end{array}\right)_{\mathrm{dis}}, \phi\right\rangle+\dot{v}(s)+\lambda_{6}(s)(v(s)-1)\right. \\
& \left.+\left\langle\phi\left(e^{i\left(\Psi-\Psi_{\infty}\right)_{2}(s, \cdot)}-1\right), \psi\right\rangle\right] d s,
\end{aligned}
$$

this leads to the following relation:

$$
\lambda_{6}(s)=-e^{-\Lambda(t)} \int_{t}^{\infty} e^{\Lambda(s)}[\ldots] d s,
$$

where [...] stands for the part on the right hand side of (3.23) without the expression $\lambda_{6}[\ldots]$. The equations 3.17, 3.18, 3.24) completely govern the evolution of the root $\operatorname{part}\left(\frac{\tilde{U}}{\tilde{U}}\right)_{\text {root }}$.

\subsection{The evolution of the dispersive part of the radiation}

We now formulate the equation satisfied by the dispersive part of the radiation, which is straightforward from Duhamel's principle. Recall from Theorem 1.3 that we need to match the initial data $\left(\begin{array}{c}U(0, \cdot) \\ U(\overline{0}, \cdot)\end{array}\right)_{\text {dis }}=\left(\begin{array}{c}A(\cdot) \\ \bar{A}(\cdot)\end{array}\right)$. To this end write

$$
\left(\begin{array}{l}
U \\
\bar{U}
\end{array}\right)(0, \cdot)=\sum_{i=1}^{6} \alpha_{i} \eta_{i, \text { proper }}+\left(\begin{array}{c}
A \\
\bar{A}
\end{array}\right) .
$$

The coefficients $\alpha_{i}$ here can be inferred from the orthogonality relations (2.2). Thus schematically 28 we get

$$
\alpha_{i}=\left\langle\left(\begin{array}{c}
U \\
\bar{U}
\end{array}\right)(0, \cdot), \xi_{k(i), \text { proper }}\right\rangle=\left\langle\left(\begin{array}{c}
\tilde{U} \\
\tilde{\tilde{U}}
\end{array}\right)(0, \cdot), \tilde{\xi}_{k(i), \text { proper }}\right\rangle .
$$

Using our standard decomposition we now get

$$
\begin{aligned}
\alpha_{i} & =\left\langle\left(\begin{array}{c}
\tilde{U} \\
\tilde{\tilde{U}}
\end{array}\right)(0, \cdot), \tilde{\xi}_{k(i), \text { proper }}(0, \cdot)\right\rangle \\
& =\sum_{j} \lambda_{j}(0)\left\langle\eta_{j, \text { proper }}, \tilde{\xi}_{k(i), \text { proper }}(0, \cdot)\right\rangle+\left\langle\left(\begin{array}{c}
\tilde{U} \\
\tilde{\tilde{U}}
\end{array}\right)_{\mathrm{dis}}(0, \cdot), \tilde{\xi}_{k(i), \text { proper }}(0, \cdot)-\xi_{k(i), \text { proper }}\right\rangle
\end{aligned}
$$

28 We really get a linear combination of expressions of the indicated form. 


$$
=\sum_{j} \lambda_{j}(0)\left\langle\eta_{j, \text { proper }}, \tilde{\xi}_{k(i), \text { proper }}(0, \cdot)\right\rangle+(v(0)-1)\left\langle\left(\begin{array}{l}
\tilde{U} \\
\overline{\tilde{U}}
\end{array}\right)_{\mathrm{dis}}(0, \cdot), \phi\right\rangle .
$$

Using this relation, we shall think of the $\alpha_{i}$ as functions of the $\lambda_{i}(0), i=1, \ldots, 6$, as well as the modulation parameters and $\tilde{U}_{\text {dis }}$. Now, to prescribe the evolution of $(\tilde{\tilde{U}})_{\text {dis }}$, we refer to (1.8). Introduce the function

$$
\begin{aligned}
\left(\begin{array}{c}
\tilde{U}^{(t)}(s, y) \\
\overline{\tilde{U}}^{(t)}(s, y)
\end{array}\right):=\left(\begin{array}{c}
e^{-i\left(\Psi-\Psi_{\infty}\right)_{1}(t)} U\left(s, y+\lambda_{\infty}\left(\mu-\mu_{\infty}\right)(t)\right) \\
e^{+i\left(\Psi-\Psi_{\infty}\right)_{1}(t)} \bar{U}\left(s, y+\lambda_{\infty}\left(\mu-\mu_{\infty}\right)(t)\right)
\end{array}\right), \\
\left(\begin{array}{c}
\tilde{U}^{(t)}(t, y) \\
\tilde{\tilde{U}}^{(t)}(t, y)
\end{array}\right)=\left(\begin{array}{c}
\tilde{U}(t, y) \\
\tilde{\tilde{U}}(t, y)
\end{array}\right) .
\end{aligned}
$$

Then we deduce the following equation:

$$
\begin{aligned}
& {\left[i \partial_{s}+\left(\begin{array}{cc}
\partial_{y}^{2}-1+3 \phi_{0}^{4} & 2 \phi_{0}^{4} \\
-2 \phi_{0}^{4} & -\partial_{y}^{2}+1-3 \phi_{0}^{4}
\end{array}\right)\right]\left(\begin{array}{l}
\tilde{U}^{(t)} \\
\tilde{\tilde{U}}^{(t)}
\end{array}\right)(s, y)} \\
& =\left(\begin{array}{cc}
e^{-i\left(\Psi-\Psi_{\infty}\right)_{1}(t)} & 0 \\
0 & e^{i\left(\Psi-\Psi_{\infty}\right)_{1}(t)}
\end{array}\right)[\ldots] \\
& +2\left(\begin{array}{cc}
0 & -e^{2 i\left(\Psi-\Psi_{\infty}\right)_{1}(s)-2 i\left(\Psi-\Psi_{\infty}\right)_{1}(t)}+1 \\
e^{-2 i\left(\Psi-\Psi_{\infty}\right)_{1}(s)+2 i\left(\Psi-\Psi_{\infty}\right)_{1}(t)}-1 & 0
\end{array}\right) \phi_{0}^{4}\left(\begin{array}{l}
\tilde{U}^{(t)}(s) \\
\tilde{\tilde{U}}^{(t)}(s)
\end{array}\right) \\
& +O\left(\left[v^{2}-1\right] \phi_{0}^{4} U\right)+O\left(\left[\mu_{\infty}-\mu\right](s) \lambda(s) \phi_{0}^{4} U\right)+O\left(e^{i\left(\Psi-\Psi_{\infty}\right)_{2}(s, \cdot)-1} \phi_{0}^{4} U\right) .
\end{aligned}
$$

The quantity $[\ldots]$ on the right hand side refers to the right hand expression in (1.8) translated by the amount $+\lambda_{\infty}\left(\mu-\mu_{\infty}\right)(t)$ in the spatial variable, but one uses the identifications

$$
4 i \kappa_{2}\left(\dot{\lambda} \lambda^{-1}-\beta v^{2}\right)=-\left\langle N, \tilde{\xi}_{5}\right\rangle+\left\langle U,\left(i \partial_{s}+\mathcal{H}(s)^{*}\right) \tilde{\xi}_{5}\right\rangle \quad \text { etc. }
$$

coming from the modulation equations, within ${ }^{29} \dot{\pi} \partial_{\pi} W$; thus we replace the left hand expressions by the ones on the right. We then project the preceding equation onto the dispersive part, and invoke Duhamel's principle, which results in the following equation governing the evolution of $\left(\frac{\tilde{U}}{\tilde{U}}\right)_{\text {dis }}$ :

$$
\begin{aligned}
& \left(\begin{array}{l}
\tilde{U} \\
\tilde{\tilde{U}}
\end{array}\right)_{\mathrm{dis}}(t, \cdot)=e^{i t \mathcal{H}} P_{s}\left[( \begin{array} { c c } 
{ e ^ { i ( \Psi _ { \infty } - \Psi ) _ { 1 } ( t ) } } & { 0 } \\
{ 0 } & { e ^ { - i ( \Psi _ { \infty } - \Psi ) _ { 1 } ( t ) } ) }
\end{array} ) \left[\left(\begin{array}{l}
A\left(\cdot+\lambda_{\infty}\left(\mu-\mu_{\infty}\right)(t)\right) \\
\bar{A}\left(\cdot+\lambda_{\infty}\left(\mu-\mu_{\infty}\right)(t)\right)
\end{array}\right)\right.\right. \\
& \left.\left.\quad+\sum_{j=1}^{6} \alpha_{j}\left[\eta_{j, \text { proper }}\left(\cdot+\lambda_{\infty}\left(\mu-\mu_{\infty}\right)(t)\right)\right]\right]\right]-i \int_{0}^{t} e^{i(t-s) \mathcal{H}}[\ldots]_{\mathrm{dis}}(s, \cdot) d s,
\end{aligned}
$$

in which [...] refers to the right hand side of 3.26]. Also, the coefficients $\alpha_{i}$ are given by the formula detailed further above, i.e.,

$$
\alpha_{i}=\sum_{j} \lambda_{j}(0)\left\langle\eta_{j, \text { proper }}, \tilde{\xi}_{k(i), \text { proper }}(0, \cdot)\right\rangle+(v(0)-1)\left\langle\left(\begin{array}{l}
\tilde{U} \\
\tilde{\tilde{U}}
\end{array}\right)_{\mathrm{dis}}(0, \cdot), \phi\right\rangle .
$$

29 Recall that we use the schematic notation $\left(i \partial_{s}+\mathcal{H}(s)\right)\left(\begin{array}{c}U \\ \bar{U}\end{array}\right)(s, \cdot)=\dot{\pi} \partial_{\pi} W+N(U, \pi)$. 


\subsection{Estimates for the radiation part}

We shall need

$$
\sup _{0 \leq k \leq[N / 3]}\left\|\langle t\rangle^{2-4 \delta_{1}} \partial_{t}^{k} \lambda_{i}(t)\right\|_{L^{M}} \lesssim \delta^{2},
$$

where $\delta_{i}, \delta, N, M$ are as in (3.14). The reason why we do not work with $L^{\infty}$ is again the compactness property 30 As for the dispersive part, let $C_{k}$ be sufficiently rapidly ${ }^{31}$ growing numbers, $1 \leq k \leq N$; we shall impose $25^{N} \delta_{2} \ll \delta_{1}$. Then we need

$$
\begin{aligned}
& \sup _{0 \leq k \leq N} \sup _{3 i+j \leq k} \sup _{s \geq 0} C_{k}^{-1}\left\|\langle s\rangle^{1 / 2-25^{k} \delta_{2}} \partial_{s}^{i} \partial_{y}^{j} U(s, y)\right\|_{L_{s}^{M} L_{y}^{M}} \lesssim \delta, \\
& \sup _{\phi \in \mathcal{A}} \sup _{0 \leq k \leq N} \sup _{3 i+j \leq k} \sup _{s \geq 0} C_{k}^{-1}\left\|\langle s\rangle^{1-20^{k} \delta_{2}} \phi \partial_{s}^{i} \partial_{y}^{j} U(s, y)\right\|_{L_{s}^{M} L_{y}^{M}} \lesssim \delta, \\
& \sup \sup _{\phi \in \mathcal{A}}\left\|\langle s\rangle^{3 / 2-\delta_{3}} \phi U(s, \cdot)\right\|_{L_{y}^{\infty}} \lesssim \delta, \quad \sup _{s \geq 0}\|C U(s, y)\|_{L_{y}^{2}} \lesssim \delta, \\
& \sup _{0 \leq k \leq N} \sup _{3 i+j \leq k} \sup _{s \geq 0} C_{k}^{-1}\left\|\langle s\rangle^{-10^{k} \delta_{2}} \partial_{s}^{i} \partial_{y}^{j} U(s, y)\right\|_{L_{s}^{M} L_{y}^{2}} \lesssim \delta,
\end{aligned}
$$

where $C$ refers to the standard pseudo-conformal operator $C=y+2 i s \partial_{y}$. Also, we denote by $\mathcal{A}$ the set of all Schwartz functions satisfying

$$
\sup _{i \leq 100 N} \sup _{x \in \mathbb{R}}\left|\langle x\rangle^{100} \partial_{x}^{i} \phi(x)\right|<1 \text {. }
$$

\section{The iteration step}

Up to this point, we have assumed the existence of a solution with the desired properties and deduced the equations governing its various parameters. Now, we need to establish the existence of such a solution with given initial data, which we do by formulating a suitable fixed point problem. First, we specify the variables we shall be working with:

Independent variables $\left\{\left(\begin{array}{c}\tilde{U} \\ \tilde{U}\end{array}\right)_{\mathrm{dis}}(\cdot, \cdot), \lambda_{1}(\cdot), \ldots, \lambda_{6}(\cdot), \nu_{1}(\cdot), \beta_{1}(\cdot), \omega_{1}(\cdot), \mu_{1}(\cdot), \gamma_{1}(\cdot)\right\}$. Here we use the following notation:

$$
\begin{aligned}
& v_{1}(s)=v(s)-1, \quad \beta_{1}(s)=\beta v-b_{\infty} \lambda_{\infty}^{-1}(s) \\
& \mu_{1}(s)=\mu(s)-\frac{2 v_{\infty} s+y_{\infty}}{a_{\infty}+b_{\infty} s}, \quad \omega_{1}(s)=\omega(s)-c_{\infty} \lambda_{\infty}^{-1}(s) \\
& \gamma_{1}(s)=\gamma(s)-s-\int_{0}^{s} c_{\infty}^{2} \lambda_{\infty}^{-2}(\lambda) d \lambda, \quad \text { where } \quad c_{\infty}=v_{\infty} a_{\infty}-\frac{b_{\infty} y_{\infty}}{2} .
\end{aligned}
$$

\footnotetext{
30 Of course we can recover $L^{\infty}$ bounds for all derivatives with exception of the top derivative from this information. We avoid this distinction in order to simplify matters.

31 As usual the necessary rate of growth can be inferred from the proof.
} 
We now explain the iterative step. We assume that we are given a tuple of independent variables, as well as the given initial data $\left({ }_{\bar{A}}^{A}\right)$ and $\lambda, \beta, \omega, \gamma, \mu$ as in Theorem 1.3 Now departing from the data, we construct a different tuple of independent variables, which we denote by prime superscripts, i.e., $\left\{\left(\frac{\tilde{U}^{\prime}}{\tilde{U}^{\prime}}\right), \ldots\right\}$, as follows:

\section{The iterative step:}

(i) Construct the 'parameters at infinity' as follows:

$$
\begin{aligned}
& a_{\infty}=\frac{\lambda}{1+v_{1}(0)}, \quad b_{\infty}=\beta \lambda-\beta_{1}(0) a_{\infty}, \quad c_{\infty}=\left[\omega-\omega_{1}(0)\right] a_{\infty}, \\
& y_{\infty}=\left[\mu(0)-\mu_{1}(0)\right] a_{\infty}, \quad v_{\infty}=\frac{c_{\infty}+b_{\infty} y_{\infty} / 2}{a_{\infty}}
\end{aligned}
$$

(ii) Construct the original modulation parameters $\lambda(s), \beta(s), \omega(s), \gamma(s), \mu(s)$ :

$$
\begin{aligned}
& \lambda(s)=\lambda_{\infty}(s)\left[v(s)_{1}(s)+1\right], \quad \beta(s)=\lambda^{-1}(s)\left[\beta_{1}(s) \lambda_{\infty}(s)+b_{\infty}\right], \\
& \mu(s)=\mu_{1}(s)+\lambda_{\infty}^{-1}(s)\left[2 v_{\infty} s+y_{\infty}\right], \\
& \omega(s)=\omega_{1}(s)+c_{\infty} \lambda_{\infty}^{-1}(s), \quad \gamma(s)=\gamma_{1}(s)+s+\int_{0}^{s} c_{\infty}^{2} \lambda_{\infty}^{-2}(\lambda) d \lambda .
\end{aligned}
$$

(iii) Construct $\Psi-\Psi_{\infty}$ as in 3.15 , where $\gamma_{\infty}$ is specified by requiring $\left(\Psi-\Psi_{\infty}\right)_{1}(0)$ $=0$.

(iv) Construct

$$
\left(\begin{array}{l}
U \\
\bar{U}
\end{array}\right)=\left(\begin{array}{cc}
e^{i\left(\Psi-\Psi_{\infty}\right)_{1}(t)} & 0 \\
0 & e^{-i\left(\Psi-\Psi_{\infty}\right)_{1}(t)}
\end{array}\right)\left(\begin{array}{l}
\tilde{U} \\
\overline{\tilde{U}}
\end{array}\right)\left(t, \cdot-\lambda_{\infty}\left(\mu-\mu_{\infty}\right)(t)\right) .
$$

Here we have

$$
\left(\begin{array}{l}
\tilde{U} \\
\overline{\tilde{U}}
\end{array}\right)(s, \cdot)=\left(\begin{array}{l}
\tilde{U} \\
\overline{\tilde{U}}
\end{array}\right)_{\mathrm{dis}}(s, \cdot)+\sum_{i=1}^{6} \lambda_{i}(s) \eta_{i, \text { proper }} .
$$

(v) Construct $\left(\begin{array}{c}\tilde{U}^{\prime} \\ \tilde{U}^{\prime}\end{array}\right)_{\text {dis }}$ by using the right hand side of 3.27). Thus all the inputs on the right hand side are with respect to the un-primed variables. One can then also define $\left(\frac{\tilde{U}_{\tilde{U}}^{(t)}}{\tilde{U}^{(t)}}\right)_{\text {dis }}$ by inserting primes on the left hand side of 3.26.

(vi) Construct the root part of $\left(\begin{array}{c}\tilde{U}^{\prime} \\ \tilde{U}^{\prime}\end{array}\right)$ : define $\lambda_{2}^{\prime}(s)$ via the right hand side of 3.17], the coefficients $\lambda_{i}^{\prime}(s), i \neq 2,6$, by means of the right hand side of (3.18), and the coefficient $\lambda_{6}^{\prime}(s)$ via the right hand side of 3.23.

(vii) Construct the first two modulation parameters $v_{1}^{\prime}(s), \beta_{1}^{\prime}(s)$ by defining $v_{1}^{\prime}(s)$ as the right hand side of (3.7), and $\beta_{1}^{\prime}(s)$ as the right hand side of 3.8). 
(viii) Construct the parameters at infinity $a_{\infty}^{\prime}, b_{\infty}^{\prime}$ via the first two formulae in (i), with $v_{1}(0)$ replaced by $v_{1}^{\prime}(0)$, and $\beta_{1}(0)$ replaced by $\beta_{1}^{\prime}(0)$. We then define $\lambda_{\infty}^{\prime}(s)=a_{\infty}^{\prime}+b_{\infty}^{\prime} s$, and from this obtain $\lambda^{\prime}(s), \beta^{\prime}(s)$ as in (ii). Further, put

$$
B^{\prime}(s)=\exp \left(\int_{0}^{s}\left[\beta^{\prime} v^{\prime 2}+\frac{1}{4 i \kappa_{2}} E_{5}\right](\sigma) d \sigma\right),
$$

where $E_{5}$ is defined with respect to the un-primed quantities as in Subsection 3.2. We shall show later that under suitable assumptions on the tuple $\left\{\left(\begin{array}{c}\tilde{U} \\ \tilde{U}\end{array}\right)_{\text {dis }}, \ldots\right\}$, we have $B^{\prime}(s)^{-1}=$ $c^{\prime} \lambda_{\infty}^{\prime-1}(s)+o(1 / s)$ for suitable $c^{\prime}$. Then define $\omega^{\prime}$ via the formula

$$
\begin{aligned}
\omega^{\prime}(s)= & c^{\prime} \lambda_{\infty}^{\prime}(s)^{-1}\left[\omega(0)+\int_{0}^{\infty} B^{\prime}(\sigma)\left[\left(i \kappa_{1}\right)^{-1} E_{3}+\frac{\beta}{2 i \kappa_{1}} E_{4}\right](\sigma) d \sigma\right] \\
& -c^{\prime} \lambda_{\infty}^{\prime}(s)^{-1} \int_{s}^{\infty} B^{\prime}(\sigma)\left[\left(i \kappa_{1}\right)^{-1} E_{3}+\frac{\beta}{2 i \kappa_{1}} E_{4}\right](\sigma) d \sigma+o\left(s^{-1}\right)
\end{aligned}
$$

(see the discussion preceding (3.11). We can infer from this a number $c_{\infty}^{\prime}$ with the property 33

$$
\omega(s)^{\prime}=\frac{c_{\infty}^{\prime}}{\lambda_{\infty}^{\prime}(s)}+o\left(\frac{1}{s}\right)
$$

whence we can define $\omega_{1}(s)^{\prime}=\omega(s)^{\prime}-c_{\infty}^{\prime} \lambda_{\infty}^{\prime-1}(s)$. Continue by setting

$$
\mu(s)^{\prime}=\mu(0)+\int_{0}^{s} \lambda_{\infty}^{\prime}(\sigma)^{-1}\left[2 \omega^{\prime} v^{\prime}+\left(i \kappa_{1} v(\sigma)\right)^{-1} E_{4}(\sigma)\right] d \sigma .
$$

Again, under suitable assumptions on the original tuple we shall be able to infer the existence of numbers $v_{\infty}^{\prime}, y_{\infty}^{\prime}$ with the property 34

$$
\mu(s)^{\prime}=\frac{2 v_{\infty}^{\prime} s+y_{\infty}^{\prime}}{a_{\infty}^{\prime}+b_{\infty}^{\prime} s}+o\left(\frac{1}{s}\right),
$$

whence we can define $\mu_{1}(s)^{\prime}=\mu(s)^{\prime}-\left(2 v_{\infty}^{\prime} s+y_{\infty}^{\prime}\right) /\left(a_{\infty}^{\prime}+b_{\infty}^{\prime} s\right)$. Also, we shall have $c_{\infty}^{\prime}=v_{\infty}^{\prime} a_{\infty}^{\prime}-b_{\infty}^{\prime} y_{\infty}^{\prime} / 2$. Finally, put

$$
\begin{array}{r}
\gamma(s)^{\prime}=\gamma(0)+\int_{0}^{s}\left[v^{\prime 2}(\sigma)-\left(2 i \kappa_{2}\right)^{-1} E_{6}(\sigma)+v^{\prime 2}(\sigma) \omega^{\prime 2}(\sigma)-\frac{1}{i \kappa_{1}} \omega(\sigma) E_{4}(\sigma)\right. \\
\left.-\frac{1}{2 \kappa_{2}}\left(i \kappa_{2}\right)^{-1} E_{2}(\sigma)\right] d \sigma
\end{array}
$$

whence we can define $\gamma_{1}(s)^{\prime}=\gamma(s)^{\prime}-s-\int_{0}^{s} c_{\infty}^{\prime 2} / \lambda_{\infty}^{\prime 2}$.

32 The quantity $B^{\prime}(s)$ is not the derivative, but the new $B(s)$.

33 We shall verify later that this definition is indeed meaningful.

34 See last footnote. 
We shall refer to the map assigning the tuple $\left\{\left(\begin{array}{c}\tilde{U}^{\prime} \\ \tilde{U}^{\prime}\end{array}\right), \ldots\right\}$ to the original tuple as $T_{A, \lambda, \beta, \omega, \gamma, \mu}$. Recall that the subscripts refer to the given data. The following proposition then shows that we may reduce the proof of Theorem 1.3 to locating a fixed point for $T_{A, \lambda, \beta, \omega, \gamma, \mu}$. We shall call a tuple of independent variables a good tuple provided it satisfies the estimates (3.14), expressed in terms of $v_{1}$ etc., as well as the estimates (3.30). In the latter, one is to think of $U$ as being expressed in terms of the variables in the tuple.

Proposition 4.1. Let $A: \mathbb{R} \rightarrow \mathbb{C}$ be a smooth function satisfying $\|A\|<\delta$ for suitably small $\delta>0$, as well as the orthogonality conditions $\left\langle\left({ }_{\bar{A}}^{A}\right), \xi_{i, \operatorname{proper}}\right\rangle=0, i=1, \ldots, 6$. Let $\left\{\left(\frac{\tilde{U}}{\tilde{U}}\right)_{\mathrm{dis}}(\cdot, \cdot), \ldots\right\}$ be a good tuple satisfying the fixed point propert $\}$

$$
T_{A, \lambda, \beta, \omega, \gamma, \mu}\left\{\left(\begin{array}{c}
\tilde{U} \\
\overline{\tilde{U}}
\end{array}\right)_{\mathrm{dis}}(\cdot, \cdot), \ldots\right\}=\left\{\left(\begin{array}{c}
\tilde{U} \\
\overline{\tilde{U}}
\end{array}\right)_{\mathrm{dis}}(\cdot, \cdot), \ldots\right\} .
$$

Also, assume that $\sup _{s \geq 0}\|U(s, \cdot)\|_{L_{x}^{2}} \lesssim \delta$ and $\sup _{s \geq 0} s^{1 / 2}\|U(s, \cdot)\|_{L_{x}^{\infty}} \lesssim \delta$. Define

$$
\begin{aligned}
\left(\begin{array}{c}
U \\
\bar{U}
\end{array}\right)(s, \cdot)= & \left(\begin{array}{cc}
e^{i\left(\Psi-\Psi_{\infty}\right)_{1}(s)} & 0 \\
0 & e^{-i\left(\Psi-\Psi_{\infty}\right)_{1}(s)}
\end{array}\right) \\
& \cdot\left[\left(\begin{array}{c}
\tilde{U} \\
\overline{\tilde{U}})_{\mathrm{dis}}+\sum_{j=1}^{6} \lambda_{j} \eta_{j, \text { proper }}
\end{array}\right]\left(s, \cdot-\lambda_{\infty}\left(\mu-\mu_{\infty}\right)(s)\right) .\right.
\end{aligned}
$$

Then the function (with $\mathcal{T}_{\infty}$ as in 1.7 )

$$
\psi(t, x)=W(t, x)+\mathcal{T}_{\infty}^{-1}\left[e^{i s} U(s, \cdot)\right](t, x)
$$

is a non-generic blow-up solution of 1.1) exploding at time $t_{*}=1 / a_{\infty} b_{\infty}$ (recall $\left.a_{\infty}, b_{\infty} \sim 1\right)$. We have

$$
W(t, x)=e^{i(\gamma(s(t))+[\omega(x-\mu)](s(t))} e^{-i \frac{\beta}{4}[\lambda(x-\mu)(s(t))]^{2}} \sqrt{\lambda(s(t))} \phi_{0}([\lambda(x-\mu)](s(t)), 1),
$$

where $s(t)=a_{\infty} t /\left(a_{\infty}^{-1}-b_{\infty} t\right)$. Finally,

$$
\left(\begin{array}{l}
U \\
\bar{U}
\end{array}\right)(0, x)=\left(\begin{array}{l}
A \\
\bar{A}
\end{array}\right)(x)+\sum_{i=1}^{6} \tilde{\lambda}_{i} \eta_{i, \text { proper }}
$$

for certain numbers $\tilde{\lambda}_{i}$ with $\left|\tilde{\lambda}_{i}\right| \lesssim \delta^{2}$.

Proof. First note that under the assumption of the proposition we have $a_{\infty}^{\prime}=a_{\infty}$ etc., and then that the modulation equations (3.6) are satisfied. We continue by verifying that $\left(\begin{array}{l}U \\ \bar{U}\end{array}\right)(s, y)$ satisfies $[1.8)$. Thus define a function $\left(\begin{array}{l}\Omega \\ \bar{\Omega}\end{array}\right)(s, y)$ by means of the inhomogeneous linear equation

\footnotetext{
35 In particular, we assume that the operation of $T_{A, \lambda, \beta, \omega, \gamma, \mu}$ is well-defined on this tuple. We shall soon analyze where this map is well-defined.

36 The function $\left(\Psi-\Psi_{\infty}\right)_{1}(s)$ is given by 3.16 .
} 


$$
\begin{aligned}
i \partial_{s}\left(\begin{array}{c}
\Omega \\
\bar{\Omega}
\end{array}\right)+\mathcal{H}(s)\left(\begin{array}{c}
\Omega \\
\bar{\Omega}
\end{array}\right)= & -i\left(\dot{\lambda} \lambda^{-1}-\beta v^{2}\right)\left(\tilde{\eta}_{2}-\beta \tilde{\eta}_{5} / 2+\omega \tilde{\eta}_{4}\right)+\frac{i}{4}\left(\dot{\beta}+\beta^{2} v^{2}\right) \tilde{\eta}_{5} \\
& +i\left(v^{2}-\dot{\gamma}+v^{2} \omega^{2}\right) \tilde{\eta}_{1}-i\left(\dot{\omega}+\beta \omega v^{2}\right) \tilde{\eta}_{4} \\
& -i v\left(\dot{\mu} \lambda_{\infty}-2 v \omega\right)\left(-\omega \tilde{\eta}_{1}-\tilde{\eta}_{3}+\beta \tilde{\eta}_{4} / 2\right)+N(U, \pi), \\
\left(\begin{array}{c}
\Omega \\
\bar{\Omega}
\end{array}\right)(0, \cdot)=\left(\begin{array}{l}
U \\
\bar{U}
\end{array}\right)(0, \cdot) . &
\end{aligned}
$$

Define

$$
\left(\begin{array}{c}
\tilde{\Omega} \\
\tilde{\tilde{\Omega}}
\end{array}\right)(t, \cdot):=\left(\begin{array}{cc}
e^{-i\left(\Psi-\Psi_{\infty}\right)_{1}(t)} & 0 \\
0 & e^{+i\left(\Psi-\Psi_{\infty}\right)_{1}(t)}
\end{array}\right)\left(\begin{array}{c}
\Omega \\
\bar{\Omega}
\end{array}\right)\left(t, \cdot+\lambda_{\infty}\left(\mu-\mu_{\infty}\right)(t)\right),
$$

and use a decomposition

$$
\left(\begin{array}{c}
\tilde{\Omega} \\
\tilde{\Omega}
\end{array}\right)(t, \cdot)=\left(\begin{array}{c}
\tilde{\Omega} \\
\tilde{\tilde{\Omega}}
\end{array}\right)_{\mathrm{dis}}(t, \cdot)+\sum_{j=1}^{6} \mu_{j}(t) \eta_{j, \text { proper. }}
$$

Now we deduce the equation

$$
\begin{aligned}
& \left(\begin{array}{c}
\tilde{\Omega} \\
\tilde{\Omega}
\end{array}\right)_{\mathrm{dis}}(t, \cdot)=e^{i t \mathcal{H}}\left(\frac{\tilde{U}^{(t)}}{\tilde{U}^{(t)}}\right)_{\mathrm{dis}}(0, \cdot)-i \int_{0}^{t} e^{i(t-s) \mathcal{H}}[\ldots]_{\mathrm{dis}} d s \\
& -2 i \int_{0}^{t} e^{i(t-s) \mathcal{H}}\left[\left(\begin{array}{cc}
0 & -e^{+2 i\left(\Psi-\Psi_{\infty}\right)_{1}(s)-2 i\left(\Psi-\Psi_{\infty}\right)_{1}(t)}+1 \\
e^{-2 i\left(\Psi-\Psi_{\infty}\right)_{1}(s)+2 i\left(\Psi-\Psi_{\infty}\right)_{1}(t)}-1 & 0
\end{array}\right)\right. \\
& \times \phi_{0}^{4}\left(\frac{\tilde{\Omega}^{(t)}}{\tilde{\Omega}^{(t)}(s, \cdot)}(s, \cdot)\right]_{\mathrm{dis}} d s-i \int_{0}^{t} e^{i(t-s) \mathcal{H}}[\widetilde{\ldots}]_{\mathrm{dis}}(s) d s,
\end{aligned}
$$

where [...] refers to the right hand side of (4.2) translated by $+\lambda_{\infty}\left(\mu-\mu_{\infty}\right)(t)$ in the

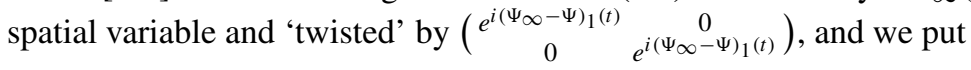

$$
\begin{aligned}
& {[\widetilde{\ldots}]=\left(\begin{array}{cc}
e^{i\left(\Psi_{\infty}-\Psi\right)_{1}(t)} & 0 \\
0 & e^{-i\left(\Psi_{\infty}-\Psi\right)_{1}(t)}
\end{array}\right)} \\
& \times\left(\begin{array}{cc}
-3 v^{2}(s) \tilde{\phi}_{0}^{4}\left(\cdot-\lambda_{\infty}\left(\mu-\mu_{\infty}\right)(s)\right)+3 \tilde{\phi}_{0}^{4} & 2 \tilde{\phi}_{0}^{4} e^{2 i\left(\Psi-\Psi_{\infty}\right)_{1}(s)}\left(-e^{2 i\left(\Psi-\Psi_{\infty}\right)_{2}(t)}+1\right) \\
-2 \tilde{\phi}_{0}^{4} e^{-2 i\left(\Psi-\Psi_{\infty}\right)_{1}(s)}\left(-e^{-2 i\left(\Psi-\Psi_{\infty}\right)_{2}(t)}+1\right) & 3 v^{2}(s) \tilde{\phi}_{0}^{4}\left(\cdot-\lambda_{\infty}\left(\mu-\mu_{\infty}\right)(s)\right)-3 \tilde{\phi}_{0}^{4}
\end{array}\right) \\
& \times\left(\begin{array}{c}
\Omega \\
\bar{\Omega}
\end{array}\right)\left(s, \cdot+\lambda_{\infty}\left(\mu-\mu_{\infty}\right)(t)\right)
\end{aligned}
$$

and as before we put $\tilde{\phi}_{0}(\cdot)=\phi\left(\cdot+\lambda_{\infty}\left(\mu-\mu_{\infty}\right)(t)\right)$. From the iterative step and the fact that the modulation equations are satisfied, we then deduce that

$$
\begin{aligned}
& \left(\frac{\tilde{\Omega}-\tilde{U}}{\tilde{\Omega}-\tilde{U}}\right)_{\mathrm{dis}}(t, \cdot) \\
& =-2 i \int_{0}^{t} e^{i(t-s) \mathcal{H}}\left[\left(\begin{array}{cc}
0 & -e^{+2 i\left(\Psi-\Psi_{\infty}\right)_{1}(s)-2 i\left(\Psi-\Psi_{\infty}\right)_{1}(t)}+1 \\
e^{-2 i\left(\Psi-\Psi_{\infty}\right)_{1}(s)+2 i\left(\Psi-\Psi_{\infty}\right)_{1}(t)}-1 & 0
\end{array}\right)\right. \\
& \left.\left.\times \phi_{0}^{4}\left(\frac{\tilde{\Omega}^{(t)}(s, \cdot)-\tilde{U}^{(t)}(s, \cdot)}{\tilde{\Omega}^{(t)}(s, \cdot)-\tilde{U}^{(t)}(s, \cdot)}\right)\right]_{\mathrm{dis}} d s-i \int_{0}^{t} e^{i(t-s) \mathcal{H}} \widetilde{\Delta[\ldots]}\right]_{\mathrm{dis}}(s) d s,
\end{aligned}
$$


where the expression $\widetilde{\Delta[\ldots]}$ dis $(s)$ is defined as above but with $\Omega$ replaced by the difference $\Omega-U$. Next, we have

$$
i \partial_{s}\left\langle\left(\begin{array}{c}
\Omega \\
\bar{\Omega}
\end{array}\right), \tilde{\xi}_{i}\right\rangle=\left\langle\left(i \partial_{s}+\mathcal{H}(s)\right)\left(\begin{array}{c}
\Omega \\
\bar{\Omega}
\end{array}\right), \tilde{\xi}_{i}\right\rangle-\left\langle\left(\begin{array}{c}
\Omega \\
\bar{\Omega}
\end{array}\right),\left(i \partial_{s}+\mathcal{H}^{*}(s)\right) \tilde{\xi}_{i}\right\rangle, \quad i=2, \ldots, 6
$$

whence we obtain

$$
i \partial_{s}\left\langle\left(\begin{array}{c}
\Omega \\
\bar{\Omega}
\end{array}\right)-\left(\begin{array}{c}
U \\
\bar{U}
\end{array}\right), \tilde{\xi}_{i}\right\rangle=-\left\langle\left(\begin{array}{c}
\Omega \\
\bar{\Omega}
\end{array}\right)-\left(\begin{array}{c}
U \\
\bar{U}
\end{array}\right),\left(i \partial_{s}+\mathcal{H}^{*}(s)\right) \tilde{\xi}_{i}\right\rangle, \quad i=2, \ldots, 6
$$

Thus we obtain from $\left(\left(\begin{array}{c}\Omega \\ \bar{\Omega}\end{array}\right)-\left(\begin{array}{c}U \\ \bar{U}\end{array}\right)\right)(0, \cdot)=0$ the relation

$$
\begin{aligned}
i\left\langle\left(\begin{array}{c}
\Omega \\
\bar{\Omega}
\end{array}\right)-\left(\begin{array}{c}
U \\
\bar{U}
\end{array}\right), \tilde{\xi}_{i}\right\rangle(t, \cdot)=\left\langle\left(\begin{array}{c}
\tilde{\Omega} \\
\overline{\tilde{\Omega}}
\end{array}\right)-\left(\begin{array}{c}
\tilde{U} \\
\overline{\tilde{U}}
\end{array}\right), \Xi_{i}\right\rangle(t, \cdot) \\
\quad=-\int_{0}^{t}\left\langle\left(\begin{array}{c}
\Omega \\
\bar{\Omega}
\end{array}\right)-\left(\begin{array}{c}
U \\
\bar{U}
\end{array}\right),\left(i \partial_{s}+\mathcal{H}^{*}(s)\right) \tilde{\xi}_{i}\right\rangle(s) d s, \quad i=2, \ldots, 6,
\end{aligned}
$$

where the first equality can be used to define $\Xi_{i}$ in the obvious fashion. We shall use the last relation to solve for the coefficients $\mu_{i}, i=1, \ldots, 5$. Finally, we consider the coefficient $\mu_{6}$ :

$$
\begin{aligned}
i \dot{\mu}_{6}(t)= & \left\langle i \partial_{t}\left(\begin{array}{c}
\tilde{\Omega} \\
\tilde{\tilde{\Omega}}
\end{array}\right), \xi_{1, \text { proper }}\right\rangle \\
= & \left\langle\left(\begin{array}{c}
\tilde{\Omega} \\
\tilde{\tilde{\Omega}}
\end{array}\right),\left(\begin{array}{cc}
\partial_{t}\left(\Psi-\Psi_{\infty}\right)_{1} & 0 \\
0 & -\partial_{t}\left(\Psi-\Psi_{\infty}\right)_{1}
\end{array}\right) \xi_{1, \text { proper }}\right\rangle \\
& +\left\langle\partial_{t}\left(\lambda_{\infty}\left(\mu-\mu_{\infty}\right)(t)\right)\left(\frac{\partial_{x} \tilde{\Omega}}{\partial_{x} \tilde{\Omega}}\right), \xi_{1, \text { proper }}\right\rangle \\
& +\left\langle\left(\frac{e^{-i\left(\Psi-\Psi_{\infty}\right)_{1}(t)} i \partial_{t} \tilde{\Omega}\left(t, y+\lambda_{\infty}\left(\mu-\mu_{\infty}\right)(t)\right)}{-e^{-i\left(\Psi-\Psi_{\infty}\right)_{1}(t)} i \partial_{t} \tilde{\Omega}\left(t, y+\lambda_{\infty}\left(\mu-\mu_{\infty}\right)(t)\right)}\right), \xi_{1, \text { proper }}\right\rangle
\end{aligned}
$$

Now we recall the corresponding identity in the derivation of (3.23), namely (3.19), take the difference of the latter and the identity above, and proceed as in the paragraphs after 3.19. Using the fact that $\lambda_{6}(0)=\mu_{6}(0)$, we deduce the schematic identity

$$
\begin{aligned}
& \left(\lambda_{6}-\mu_{6}\right)(t) \\
& =\int_{0}^{t}\left[\left\langle\left(\begin{array}{c}
\tilde{\Omega} \\
\tilde{\tilde{\Omega}}
\end{array}\right)(s, \cdot)-\left(\begin{array}{c}
\tilde{U} \\
\overline{\tilde{U}}
\end{array}\right)(s, \cdot), \phi(s)\right\rangle+\left\langle\partial_{x}\left(\begin{array}{c}
\tilde{\Omega} \\
\overline{\tilde{\Omega}}
\end{array}\right)(s, \cdot)-\partial_{x}\left(\begin{array}{c}
\tilde{U} \\
\overline{\tilde{U}}
\end{array}\right)(s, \cdot), \psi(s)\right\rangle\right] d s .
\end{aligned}
$$

Now from 4.4, 4.5, 4.6, as well as 4.3 and the linear estimate Theorem 2.1, we easily deduce the estimate 


$$
\begin{aligned}
\sup _{0 \leq t \leq T}\left[\left\|[\tilde{\Omega}-\tilde{U}]_{\operatorname{dis}}(t, \cdot)\right\|_{H^{1}}\right. & \left.+\sum_{i=1}^{6}\left|\lambda_{i}-\mu_{i}\right|(t)\right] \\
& \lesssim T \sup _{0 \leq t \leq T}\left[\left\|[\tilde{\Omega}-\tilde{U}]_{\operatorname{dis}}(t, \cdot)\right\|_{H^{1}}+\sum_{i=1}^{6}\left|\lambda_{i}-\mu_{i}\right|(t)\right] .
\end{aligned}
$$

Now choose $T>0$ small enough to get the identity $\left.\Omega\right|_{[0, T]}=\left.U\right|_{[0, T]}$. Continuing in this fashion implies $U(\cdot, \cdot)=\Omega(\cdot, \cdot)$. Next, observe that the condition

$$
\begin{array}{r}
\left(\begin{array}{c}
\tilde{U} \\
\overline{\tilde{U}}
\end{array}\right)_{\mathrm{dis}}(0, \cdot)=P_{S}\left[\sum_{i} \eta_{i, \operatorname{proper}}\left(\cdot+\lambda_{\infty}\left(\mu-\mu_{\infty}\right)(0)\right)\left\langle\left(\begin{array}{c}
\tilde{U} \\
\overline{\tilde{U}}
\end{array}\right)(0, \cdot), \tilde{\xi}_{k(i), \text { proper }}\right\rangle\right. \\
\left.+\left(\begin{array}{c}
A \\
\bar{A}
\end{array}\right)\left(\cdot+\lambda_{\infty}\left(\mu-\mu_{\infty}\right)(0)\right)\right]
\end{array}
$$

in addition to $P_{\text {root }}\left(\begin{array}{c}\tilde{U} \\ \tilde{U}\end{array}\right)(0, \cdot)=\sum_{i=1}^{6} \lambda_{i}(0) \eta_{i}$,proper uniquely determines $\left(\begin{array}{c}\tilde{U} \\ \tilde{U}\end{array}\right)_{\text {dis }}(0, \cdot)$, hence in conjunction with the values of the modulation parameters also $(\underset{\bar{U}}{U})(0, \cdot)$. Then one verifies that $\left(\begin{array}{c}U \\ \bar{U}\end{array}\right)(0, \cdot)=\left(\begin{array}{c}A \\ { }_{\bar{A}}\end{array}\right)+\sum_{i} \alpha_{i} \eta_{i}$,proper with the $\alpha_{i}$ defined as in 3.28) is consistent with 4.7 , as well as the root part of $(\underset{\tilde{U}}{\tilde{U}})(0, \cdot)$.

Now reverse the algebraic manipulations that led to 1.8 . We deduce that

$$
Z(t, x)=W(t, x)+\mathcal{T}_{\infty}^{-1}\left[e^{i s} U(s, \cdot)\right](t, x)
$$

is indeed a non-generic blow-up solution of (1.1). One checks that

$$
\begin{aligned}
& \mathcal{T}_{\infty}^{-1}\left[e^{i s} U(s, \cdot)\right]=\left(a_{\infty}^{-1}-b_{\infty} t\right)^{-1 / 2} e^{-i \frac{b_{\infty} x^{2}}{4\left(a_{\infty}^{-1}-b_{\infty} t\right)}} e^{i\left(\gamma_{\infty}+v_{\infty} x-v_{\infty}^{2} \frac{a_{\infty} t}{a_{\infty}^{-1}-b_{\infty} t}-v_{\infty} y_{\infty}\right) i\left(\frac{a_{\infty} t}{a_{\infty}^{-1}-b_{\infty} t}\right)} \\
& \cdot U\left(\frac{a_{\infty} t}{a_{\infty}^{-1}-b_{\infty} t}, \frac{x-2 v_{\infty} a_{\infty} t}{a_{\infty}^{-1}-b_{\infty} t}-y_{\infty}\right) .
\end{aligned}
$$

The assumptions in the proposition imply that this remains bounded with respect to $L^{\infty}$ as $t \rightarrow t_{*}=1 / a_{\infty} b_{\infty}$, while due to the asymptotic relations (3.14) the principal soliton part $W(t, x)$ blows up according to the non-generic profile. Finally, recall the decomposition (3.25) in which we use (3.28). Our assumptions (3.14) as well as 3.29) imply the last statement of the proposition.

\subsection{Deducing the fixed point from a priori estimates}

We now need to demonstrate the existence of a fixed point for the map $T_{A}$ on the set of tuples satisfying the above specified inequalities. This will follow from an application of the Schauder-Tikhonov Fixed Point Theorem, which we recall here:

Theorem 4.2 (Schauder-Tikhonov). A non-empty compact convex subset $S$ of a Banach space has the fixed point property, i.e., for any continuous map $T: S \rightarrow S$ there exists $x_{T} \in S$ satisfying $T\left(x_{T}\right)=x_{T}$. 
We now need to locate such a set $S$. We construct it as follows: first, for $M, N$ as before (3.14,, 3.29 ) and very large ${ }^{37} K>0$ introduce the norm 38

$$
\begin{aligned}
& \|U\|_{S^{N, K}}=\sum_{0 \leq k \leq N} C_{k}^{-1} \sum_{3 i+j \leq k}\left[\sup _{s \geq 0}\langle s\rangle^{1 / 2-25^{k} \delta_{2}}\left\|\partial_{s}^{i} \partial_{y}^{j} U(s, y)\right\|_{L_{s}^{M} L_{y}^{M}}\right. \\
& \left.+\sup _{s \geq 0}\langle s\rangle^{-10^{k} \delta_{2}}\left\|\partial_{s}^{i} \partial_{y}^{j} U(s, y)\right\|_{L_{s}^{M} L_{y}^{2}}+\sup _{\phi \in \mathcal{A}} \sup _{s \geq 0}\langle s\rangle^{1-20^{k} \delta_{2}}\left\|\phi \partial_{s}^{i} \partial_{y}^{j} U(s, y)\right\|_{L_{s}^{M} L_{y}^{M}}\right] \\
& +\sup _{s \geq 0}\left[\sup _{\phi \in \mathcal{A}}\langle s\rangle^{3 / 2-\delta_{3}}\|\phi U(s, \cdot)\|_{L_{y}^{\infty}}+\sup _{s \geq 0}\|C U(s, y)\|_{L_{y}^{2}}\right. \\
& \left.+\sum_{1 \leq k \leq N-1} K^{-k} \sup _{3 i+j=k}\left\|C \partial_{s}^{i} \partial_{y}^{j} U(s, \cdot)\right\|_{L_{s}^{M} L_{y}^{2}}\right],
\end{aligned}
$$

where $C$ is as in 3.30 . The role of the last summand is to ensure uniform spatial decay on finite time intervals, again needed for compactness. Also, let $\left|\|U \mid\|_{S^{N}}\right.$ be defined as above, but with the last summand replaced by $\|C U\|_{L_{y}^{2}}$, where we use $C=y+2 i s \partial_{y}$. Define the Banach spaces $S^{N, K}$ and $S^{N}$ as the completions of $\mathcal{S}\left(\mathbb{R}^{2}\right)$ with respect to these norms. Now for a tuple $\Gamma:=\left\{\left(\frac{\tilde{U}}{\tilde{U}}\right)_{\text {dis }}, \ldots\right\}$ as before, define the norm ${ }^{39}$ (as usual we let $\langle s\rangle=|s|+1)$

$$
\begin{aligned}
& \|\Gamma \mid\| \tilde{S}_{\tilde{S}^{N, K}}:=\left\|\tilde{U}_{\mathrm{dis}}\right\|_{S^{N, K}} \\
& +\delta^{-1} \sup _{0 \leq s<\infty}\langle s\rangle^{1 / 2-\delta_{1}}\left|\nu_{1}(s)\right|+\sum_{1 \leq k \leq[N / 3]}\left\|\langle s\rangle^{3 / 2-2 \delta_{1}} \frac{d^{k}}{d s^{k}} v_{1}(s)\right\|_{L^{M}} \\
& +\sup _{0 \leq s<\infty}\langle s\rangle^{3 / 2-\delta_{1}}\left|\beta_{1}(s)\right|+\sum_{1 \leq k \leq[N / 3]}\left\|\langle s\rangle^{2-2 \delta_{1}} \frac{d^{k}}{d s^{k}} \beta_{1}(s)\right\|_{L^{M}}+\sup _{0 \leq s<\infty}\left|\langle s\rangle^{3 / 2-\delta_{1}} \omega_{1}(s)\right| \\
& +\sum_{1 \leq k \leq[N / 3]}\left\|\langle s\rangle^{3 / 2-2 \delta_{1}} \frac{d^{k}}{d s^{k}} \omega_{1}(s)\right\|_{L^{M}}+\sup _{0 \leq s<\infty}\left|\langle s\rangle^{1 / 2-\delta_{1}} \frac{d}{d s} \gamma_{1}(s)\right|_{0 \leq s<\infty} \|\langle s\rangle^{3 / 2-\delta_{1}}\left|\mu_{1}(s)\right| \\
& +\sum_{2 \leq k \leq[N / 3]}\left\|\langle s\rangle^{3 / 2-2 \delta_{1}} \frac{d^{k}}{d s^{k}} \gamma_{1}(s)\right\|_{L^{M}}+\sup _{0 \leq s<\infty} \|\left\langle\sum_{i=1}\left\|\langle t\rangle^{2-4 \delta_{1}} \frac{d^{k}}{d t^{k}} \lambda_{i}(t)\right\|_{L^{M}}\right] .
\end{aligned}
$$

Also, let $\||\Gamma|\| \tilde{S}_{\tilde{S}^{N}}$ be defined as above but with $\left\|\tilde{U}_{\mathrm{dis}}\right\|_{S^{N, K}}$ replaced by $\left\|\tilde{U}_{\mathrm{dis}}\right\|_{S^{N}}$. Then we define the restrictions \|\|$\cdot \|_{S^{N}([0, T))}$ etc. for any time interval $[0, T)$ in the obvious

37 This parameter will eventually depend on a time $T$.

38 We only include the parameters $N, K$ as superscripts in the norm, since we shall only vary these.

39 We use the notation $\left(\begin{array}{c}\tilde{U} \\ \tilde{U}\end{array}\right)_{\text {dis }}=\left(\begin{array}{c}\tilde{U}_{\text {dis }} \\ \tilde{\tilde{U}}_{\text {dis }}\end{array}\right)$. 
fashion, and denote by $S^{N}([0, T))$ and $\tilde{S}^{N, K}([0, T))$ the completions of $\mathcal{S}([0, T) \times \mathbb{R})$ and of

$$
\mathcal{S}([0, T) \times \mathbb{R}) \times\left(C^{\infty}[0, T)\right)^{11},
$$

respectively, with respect to the above norms localized to $[0, T) \times \mathbb{R}$. Then we have 40

Lemma 4.3. Fix $0<T<\infty$. For any $R \geq 0$, the set of tuples $A_{[0, T)}^{(0)}:=\{\Gamma$ on $\left.[0, T) \times \mathbb{R} \mid\|\Gamma\|_{\tilde{S}^{N, K}[0, T)} \leq R \delta\right\}$ equipped with the norm $\|\cdot\| \|_{\tilde{S}^{N-4, K}[0, T)}$ is a compact convex subset of

$$
A_{[0, T)}:=\left\{\Gamma \text { defined on }[0, T) \times \mathbb{R} \mid\|\Gamma\|_{\tilde{S}^{N-4, K}[0, T)} \leq R \delta\right\} .
$$

Proof. We demonstrate the compactness assertion: thus consider a sequence of tuples $\left\{\Gamma_{i}\right\}_{i \geq 1} \subset A_{[0, T)}^{(0)}$. Consider the functions

$$
\left(\begin{array}{c}
\tilde{U}_{i} \\
\tilde{\tilde{U}}_{i}
\end{array}\right)_{\mathrm{dis}}=\left(\frac{\tilde{U}_{i, \mathrm{dis}}}{\tilde{U}_{i, \mathrm{dis}}}\right)
$$

By assumption, letting $\phi_{\rho}(x):=\phi(x / \rho)$, where $\phi(\cdot)$ smoothly localizes to $|x|>1$, we have

$$
\lim _{\rho \rightarrow \infty} \sup _{l} \sum_{0 \leq k \leq N-4} \sum_{3 i+j \leq k}\left\|\phi_{\rho}(x) \partial_{t}^{i} \partial_{x}^{j} \tilde{U}_{l, \mathrm{dis}}\right\|_{L_{t}^{M} L_{x}^{2}[0, T)}=0 .
$$

Indeed, this follows from uniform control over $\left\|C \partial_{t}^{i} \partial_{x}^{j} \tilde{U}_{l, \mathrm{dis}}\right\|_{L_{t}^{M} L_{x}^{2}[0, T)}$. Combining this with the fact that $\sum_{0 \leq k \leq N} \sup _{3 i+j \leq k}\left\|\partial_{s}^{i} \partial_{x}^{j} \tilde{U}_{i, \text { dis }}\right\|_{L_{s}^{M} L_{x}^{2}[0, T)}$ is uniformly bounded and applying the Rellich-Kondrashov Compactness Theorem as well as Sobolev embedding, we obtain a subsequence (which we again label $\left(\begin{array}{l}\tilde{U}_{i} \\ \tilde{U}_{i}\end{array}\right)_{\text {dis }}$ ) which converges with respect to $\sum_{0 \leq k \leq N-4} \sup _{3 i+j \leq k}\left\|\partial_{s}^{i} \partial_{x}^{j}(\cdot)\right\|_{L_{s}^{M} L_{x}^{2}[0, T)}$ as well as the remaining norms in $\|\cdot\|_{S^{N-4, K}}$ to a $\operatorname{limi} 42(\underset{\tilde{U}}{\tilde{U}})_{\text {dis }}$. Passing to a further subsequence, we may assume that all $\partial_{s}^{i} \partial_{x}^{j} \tilde{U}_{i, \text { dis }}$, $N \geq 3 i+j \geq N-4$, converge weakl $\sqrt{43}$ with respect to $L_{s}^{M} L_{x}^{2}[0, T), L_{s}^{M} L_{x}^{M}[0, T)$, and one checks that the corresponding limits necessarily equal $\partial_{s}^{i} \partial_{x}^{j} \tilde{U}_{\text {dis }}$ in the distribution sense, respectively. Also, $\|\left.\left|\left(\frac{\tilde{U}}{\tilde{U}}\right)_{\text {dis }}\right|||\right|_{S^{N}} \leq R \delta$.

Now consider the root part, i.e., the functions $\lambda_{j, i}(t), j=1, \ldots, 6$. By assumption, we have a uniform bound on $\sum_{0 \leq k \leq[N / 3]}\left\|\langle t\rangle^{2-4 \delta_{1}} \frac{d^{k}}{d t^{k}} \lambda_{j, i}(t)\right\|_{L^{M}[0, T)}$. By the Arzelà-Ascoli Theorem, we can then choose a converging subsequence with respect to $\sum_{0 \leq k \leq[N / 3]-2}\left\|\langle t\rangle^{2-4 \delta_{1}} \frac{d^{k}}{d t^{k}}(\cdot)\right\|_{L^{M}[0, T)}$ whose limit satisfies the desired estimates. The argument for the modulation parameters $v_{1}(t)=v(t)-1$ etc. is identical.

40 Recall that the first entry of a tuple is always required to also satisfy the orthogonality conditions.

41 We omit the dependence of these sets on $R$ in the notation, it being understood that $R$ below will be fixed throughout.

42 Clearly this limit satisfies the same orthogonality relations, whence we may apply the subscript "dis".

43 It is at this stage that we need $L^{M}$ instead of $L^{\infty}$. 
Now define the sets $A_{[0, T)}^{(n)}, n \geq 1$, inductively as follows: first, we can modify the inductive step $T_{A, \lambda, \beta, \omega, \gamma, \mu}$ to the interval $[0, T)$, by simply replacing $\infty$ by $T$ in the formulae for the modulation parameters and root parameters. By abuse of notation refer to this by $T_{A, \lambda, \beta, \omega, \gamma, \mu}$ as well. Then put

$$
A_{[0, T)}^{(n)}:=\operatorname{convhull}\left[A_{[0, T)}^{(0)} \cap \overline{\left.T_{A, \lambda, \beta, \omega, \gamma, \mu}\left(A_{[0, T)}^{(n-1)}\right)\right]} .\right.
$$

The closure operation is always with respect to $\|\cdot\| \|_{\tilde{S}^{N-4, K}}$. Then clearly $A_{[0, T)}^{(n)} \subset A_{[0, T)}^{(n-1)}$, and these are all compact convex subsets of $A_{[0, T)}$.

Everything now reduces to the core analytic theorem below. First we make a definition:

Definition 4.4. We call a function $A: \mathbb{R} \rightarrow \mathbb{C}$ admissible provided

$$
\|A\|:=\sup _{0 \leq k \leq N}\left\|\langle x\rangle^{100} \frac{d^{k}}{d x^{k}} A(x)\right\|_{L^{1} \cap L^{2}} \leq \delta .
$$

Theorem 4.5. Let $A$ be admissible. Let $N$ satisfy the specifications in (3.14), (3.30), and $\delta>0$ be small enough. There exists $R>0$ sufficiently large such that with the corresponding $A_{[0, T)}^{(0)}$ etc. constructed as above we have the following: For every $T>0$, there exists a number $K=K(N, T)$ as well as an index $n_{0}(N)$ such that for $n \geq n_{0}$, we have $T_{A, \lambda, \beta, \omega, \gamma, \mu}\left(A_{[0, T)}^{(n)}\right) \subset A_{[0, T)}^{(n)}$; moreover, $T_{A, \lambda, \beta, \omega, \gamma, \mu}$ acts continuously on $A_{[0, T)}^{(n)}$. The last assertions are always non-vacuous if $\delta>0$ is sufficiently small, since then $A_{[0, T)}^{(n)} \neq \emptyset$. Thus by Theorem 4.2 there exists a tuple $\Gamma_{T} \in A_{[0, T)}^{(n)}$ with the property $T_{A, \lambda, \beta, \omega, \gamma, \mu} \Gamma_{T}=\Gamma_{T}$. For $T<T$, one has the inequality

$$
\left.\left\|\left.|| \Gamma_{\tilde{T}}\right|_{[0, T) \times \mathbb{R}}\right\|\right|_{\tilde{S}^{N, K(N, T)}} \lesssim \delta .
$$

Also, we get the uniform ${ }^{44}$ bounds

$$
\sup _{T>s \geq 0}\left\|\tilde{U}_{T}(s, \cdot)\right\|_{L_{x}^{2}} \lesssim \delta, \quad \sup _{T>s \geq 0}\langle s\rangle^{1 / 2}\left\|\tilde{U}_{T}(s, \cdot)\right\|_{L_{x}^{\infty}} \lesssim \delta .
$$

Assuming this, we can now deduce the following:

Theorem 4.6. There exists a fixed point $\Gamma$ for $T_{A}$ (acting on $[0, \infty) \times \mathbb{R}$ ) satisfying the assumptions (3.14), 3.29), 3.30). Thus the assumption of Proposition 4.1 is realizable.

Proof. Let $T_{i}=i, i \geq 1$. Then construct fixed points $\Gamma_{i}$ for the operation of $\left.T_{A}\right|_{\left[0, T_{i}\right]}$ as in the preceding theorem. Thanks to the uniform bounds for $\left\|\left|\Gamma_{i}\right|_{[0, j)} \mid\right\|_{\tilde{S}^{N, K\left(N, T_{j}\right)}}, j \leq i$, and invoking another compactness argument as before, we can select a subsequence $\Gamma_{j, i}$ which converges on $\left[0, T_{j}\right)$ with respect to $\|\mid \cdot\| \tilde{S}_{\tilde{S}^{N-4, K(N, j)}}$. Observe that we only need a uniform bound on $K(N, T)$ for bounded $T$ here, as we have arranged. Doing this for $j=$ $1,2, \ldots$ and invoking the Cantor diagonal argument, we then construct a subsequence, which we again label $\Gamma_{i}$, which converges on every $\left[0, T_{j}\right], j \geq 1$, to a tuple in $\tilde{S}^{N, K(N, j)}$. The limits then fit coherently to define a tuple $\Gamma$ on $[0, \infty)$ living in $\tilde{S}^{N}$, which is the desired fixed point.

\footnotetext{
44 The implied constant is independent of $T$.
} 


\section{The proof of the core analytic estimates, Theorem 4.5}

\subsection{First stage. Estimates for the radiation part}

We shall show that $T_{A, \lambda, \beta, \omega, \gamma, \mu}\left(A_{[0, T)}^{(n)}\right) \subset A_{[0, T)}^{(0)}$, provided $n$ is large enough. The proof will also reveal the continuity of the operation $T_{A, \lambda, \beta, \omega, \gamma, \mu}$. Also, we shall show that if one iterates $T_{A, \lambda, \beta, \omega, \gamma, \mu}$ starting with the tuple $\Gamma_{\text {trivial }}:=\left\{\left(\begin{array}{l}0 \\ 0\end{array}\right), 0, \ldots, \lambda(0), \beta(0), 0,0,0\right\}$, one always stays inside $A_{[0, T)}^{(0)}$ provided $\delta, \delta_{i}$ etc. are chosen suitably, whence $A_{[0, T)}^{(i)} \neq \varnothing$ for all $i \geq 0$. Then observe that

$$
T_{A, \lambda, \beta, \omega, \gamma, \mu}\left(A_{[0, T)}^{(n+1)}\right) \subset A_{[0, T)}^{(0)}
$$

as well as

$$
\begin{aligned}
& T_{A, \lambda, \beta, \omega, \gamma, \mu}\left(\operatorname{convhull}\left(\overline{T_{A, \lambda, \beta, \omega, \gamma, \mu} A_{[0, T)}^{(n)}}\right)\right) \\
&\left.\subset T_{A, \lambda, \beta, \omega, \gamma, \mu}\left(\operatorname{convhull} \overline{\left(T_{A, \lambda, \beta, \omega, \gamma, \mu} A_{[0, T)}^{(n-1)}\right.} \cap A_{[0, T)}^{(0)}\right)\right) \\
& \subset \operatorname{convhull}\left(\overline{T_{A, \lambda, \beta, \omega, \gamma, \mu}\left(A_{[0, T)}^{(n)}\right)}\right) .
\end{aligned}
$$

This then implies $T_{A, \lambda, \beta, \omega, \gamma, \mu}\left(A_{[0, T)}^{(n+1)}\right) \subset A_{[0, T)}^{(n+1)}$, as desired. Thus we need to prove

Theorem 5.1. Under the assumptions of Theorem 4.5. $T_{A, \lambda, \beta, \omega, \gamma, \mu}\left(A_{[0, T)}^{(n)}\right) \subset A_{[0, T)}^{(0)}$.

Proof. This shall occupy the rest of the paper. We first interpret the condition $\Gamma_{T} \in$ $A_{[0, T)}^{(n)}$. Note that by definition this means that $\Gamma_{T}$ is the limit of finite convex linear combinations $\sum_{\alpha_{i} \geq 0, \sum_{i} \alpha_{i}=1} \alpha_{i} \Gamma_{T}^{(i)}$ where each $\Gamma_{T}^{(i)}$ itself is in intersection of the image of $T_{A, \lambda, \beta, \omega, \gamma, \mu}$ applied to $A_{[0, T)}^{(n-1)}$ and the set $A_{[0, T)}^{(0)}$. It will suffice to restrict the proof to tuples which are obtained by iteration of alternately forming convex linear combinations and applying $T_{A, \lambda, \beta, \omega, \gamma, \mu}$. In order to proceed, we will need the following two lemmas in order to control the phase function $\Psi-\Psi_{\infty}$ :

Lemma 5.2. Assume that the relations (3.14) are satisfied. Then for $\phi \in \mathcal{A}$ (recall the definitions after (3.30) we have

$$
\phi(y)\left|\left(\Psi-\Psi_{\infty}\right)_{2}\right|(t, y) \lesssim \delta^{2}\langle t\rangle^{-3 / 2+\delta_{1}} .
$$

Proof. Recall the definition

$$
\begin{aligned}
\left(\Psi-\Psi_{\infty}\right)_{2}(t, y)= & y\left[\omega(t) v(t)-\frac{\beta(t)}{2} v(t) \lambda(t)\left(\mu_{\infty}-\mu\right)(t)-\frac{a_{\infty} v_{\infty}-b_{\infty} y_{\infty} / 2}{a_{\infty}+b_{\infty} t}\right] \\
& +y^{2}\left[\frac{b_{\infty}}{4\left(a_{\infty}+b_{\infty} t\right)}-\frac{\beta(t)}{4} v^{2}(t)-\frac{\beta(t)}{4} \lambda^{2}(t)\left(\mu_{\infty}-\mu\right)^{2}(t)\right] .
\end{aligned}
$$

The claimed estimate now follows easily from the facts that $c_{\infty}=a_{\infty} v_{\infty}-b_{\infty} y_{\infty} / 2$ as well as $\left|\omega(t)-c_{\infty} / \lambda_{\infty}(t)\right| \lesssim \delta^{2}\langle t\rangle^{-3 / 2+\delta_{1}},\left|\beta(t) v(t)-b_{\infty} / \lambda_{\infty}(t)\right| \lesssim \delta^{2}\langle t\rangle^{-3 / 2+\delta_{1}}$, $|v(t)-1| \lesssim \delta^{2}\langle t\rangle^{-1 / 2+\delta_{1}}$. 
We also need

Lemma 5.3. The following estimate holds under the same assumptions as in the preceding lemma:

$$
\left|\left(\Psi-\Psi_{\infty}\right)_{1}(t)\right| \lesssim \delta^{2}\langle t\rangle^{1 / 2+\delta_{1}} .
$$

Proof. This is along the same lines, although the algebra is a bit more complicated. Observe that

$\frac{d}{d s}\left[\frac{v_{\infty}^{2} s a_{\infty}}{a_{\infty}+b_{\infty} s}-\frac{b_{\infty} v_{\infty} s y_{\infty}}{a_{\infty}+b_{\infty} s}+\gamma_{\infty}-\frac{b_{\infty} y_{\infty}^{2}}{4\left(a_{\infty}+b_{\infty} s\right)}\right]=\frac{\left(a_{\infty} v_{\infty}-b_{\infty} y_{\infty} / 2\right)^{2}}{\left(a_{\infty}+b_{\infty} s\right)^{2}}=\frac{c_{\infty}^{2}}{\lambda_{\infty}^{2}(s)}$, while also $\left|\partial_{s}\left[\gamma_{s}-s\right]-c_{\infty} \lambda_{\infty}^{-2}(s)\right| \lesssim \delta^{2} s^{-1 / 2+\delta_{1}}$. The claim follows easily from this and the definition of $\left(\Psi-\Psi_{\infty}\right)_{1}$.

We commence by controlling the dispersive part of the radiation, $\left(\begin{array}{c}\tilde{U}^{\prime} \\ \tilde{U}^{\prime}\end{array}\right)_{\mathrm{dis}}$, first dealing with the easier estimates:

Lemma 5.4. Let $\Gamma_{T} \in A_{[0, T)}^{(1)}$. Then provided the conditions of Theorem 4.5 are satisfied, we have

$$
\begin{aligned}
& \sum_{0 \leq k \leq N} C_{k}^{-1} \sum_{3 i+j \leq k}\left[\sup _{s \geq 0}\langle s\rangle^{1 / 2-25^{k} \delta_{2}}\left\|\partial_{s}^{i} \partial_{y}^{j} \tilde{U}_{\mathrm{dis}}^{\prime}(s, y)\right\|_{L_{s}^{M} L_{y}^{M}}\right. \\
& \left.+\sup _{s \geq 0}\langle s\rangle^{-10^{k} \delta_{2}}\left\|\partial_{s}^{i} \partial_{y}^{j} \tilde{U}_{\mathrm{dis}}^{\prime}(s, y)\right\|_{L_{s}^{M} L_{y}^{2}}+\sup _{\phi \in \mathcal{A}} \sup _{s \geq 0}\langle s\rangle^{1-20^{k} \delta_{2}}\left\|\phi \partial_{s}^{i} \partial_{y}^{j} \tilde{U}_{\mathrm{dis}}^{\prime}(s, y)\right\|_{L_{s}^{M} L_{y}^{M}}\right] \leq \frac{R}{2} \delta,
\end{aligned}
$$

where as usual $T_{A, \lambda, \beta, \omega, \gamma, \mu} \Gamma_{T}=\Gamma_{T}^{\prime}$.

Remark 5.5. Note that we do not need the nested structure implicit in the definition of the $A_{[0, T)}^{(n)}$ for these estimates.

Proof. It suffices to prove the assertion for a tuple $\Gamma_{T}^{\prime}=T_{A, \lambda, \beta, \omega, \gamma, \mu} \Gamma_{T}$. We start with the case $k=0$ by controlling $C_{0}^{-1}\langle s\rangle^{1 / 2-\delta_{2}}\left\|\tilde{U}_{\text {dis }}^{\prime}\right\|_{L_{y}^{M}}$. Now use 3.27] as well as Duhamel's principle and Theorem 2.1 to deduce that

$$
\begin{aligned}
C_{0}^{-1}\langle t\rangle^{1 / 2-\delta_{2}}\left\|\left(\begin{array}{l}
\tilde{U}^{\prime} \\
\tilde{\tilde{U}}^{\prime}
\end{array}\right)_{\mathrm{dis}}(t, \cdot)\right\|_{L_{x}^{M}} & C_{0}^{-1}\langle t\rangle^{1 / 2-\delta_{2}} \| e^{i t \mathcal{H}} P_{s}\left[( \begin{array} { c c } 
{ e ^ { - i ( \Psi - \Psi _ { \infty } ) _ { 1 } ( t ) } } & { 0 } \\
{ 0 } & { e ^ { i ( \Psi - \Psi _ { \infty } ) _ { 1 } ( t ) } ) }
\end{array} ) \left[\left(\begin{array}{l}
A\left(\cdot+\lambda_{\infty}\left(\mu-\mu_{\infty}\right)(t)\right) \\
\bar{A}\left(\cdot+\lambda_{\infty}\left(\mu-\mu_{\infty}\right)(t)\right)
\end{array}\right)_{\mathrm{dis}}\right.\right. \\
& \left.\left.+\sum_{j=1}^{6} \alpha_{j} \eta_{j, \text { proper }}\left(\cdot+\lambda_{\infty}\left(\mu-\mu_{\infty}\right)(t)\right)\right]\right] \|_{L_{x}^{M}} \\
& +C_{0}^{-1}\langle t\rangle^{1 / 2-\delta_{2}} \int_{0}^{t}\left\|e^{i(t-s) \mathcal{H}}[\ldots]_{\mathrm{dis}}(s, \cdot)\right\|_{L_{x}^{M}} d s .
\end{aligned}
$$

The first two terms here are easy to estimate with respect to $\|\cdot\|_{L_{t}^{M}}$ on account of 3.14 as well as Theorem 2.1 if one chooses $R$ large enough. Indeed, one gets the bound $\frac{R}{100} \delta$ by choosing $R$ large enough in relation to $\|A\|$. We now turn to estimating the terms 
implicit in $[\ldots]_{\text {dis. }}$ Recalling 3.26 as well as the estimates implied by $\Gamma_{T} \in A_{[0, T)}^{(1)}$, we see that the term linear in $U$ and of least temporal decay is the following:

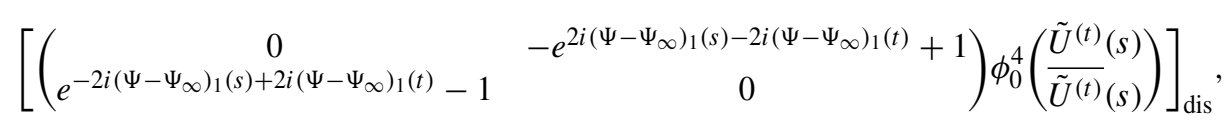

whence we need to estimate

$$
\begin{gathered}
C_{0}^{-1}\langle t\rangle^{1 / 2-\delta_{2}} \\
\cdot \int_{0}^{t} \| e^{i(t-s) \mathcal{H}}\left[\left(\begin{array}{cc}
0 & -e^{2 i\left(\Psi-\Psi_{\infty}\right)_{1}(s)-2 i\left(\Psi-\Psi_{\infty}\right)_{1}(t)}+1 \\
e^{-2 i\left(\Psi-\Psi_{\infty}\right)_{1}(s)+2 i\left(\Psi-\Psi_{\infty}\right)_{1}(t)}-1 & 0
\end{array}\right)\right. \\
\\
\left.\phi_{0}^{4}\left(\frac{\tilde{U}^{(t)}(s)}{\tilde{U}^{(t)}(s)}\right)\right]_{\mathrm{dis}} \|_{L_{x}^{M}} d s .
\end{gathered}
$$

Observe from Lemma 5.3 that

$$
\sup _{0 \leq s \leq \delta^{-1 / 2}}\left|\left(\Psi-\Psi_{\infty}\right)_{1}\right|(s) \lesssim R \delta^{7 / 4-\delta_{1}}
$$

whence we get, if we restrict $t<\delta^{-1 / 2}$ and $M \gg \delta_{2}^{-1}$,

$$
\begin{aligned}
& C_{0}^{-1} \| \chi_{<\delta^{-1 / 2}}(t)\langle t\rangle^{1 / 2-\delta_{2}} \\
& \cdot \int_{0}^{t} \| e^{i(t-s) \mathcal{H}}\left[\left(\begin{array}{cc}
0 & -e^{2 i\left(\Psi-\Psi_{\infty}\right)_{1}(s)-2 i\left(\Psi-\Psi_{\infty}\right)_{1}(t)}+1 \\
e^{-2 i\left(\Psi-\Psi_{\infty}\right)_{1}(s)+2 i\left(\Psi-\Psi_{\infty}\right)_{1}(t)}-1 & 0
\end{array}\right)\right. \\
& \left.\cdot \phi_{0}^{4}\left(\frac{\tilde{U}^{(t)}(s)}{\tilde{U}^{(t)}(s)}\right)\right]_{\mathrm{dis}}\left\|_{L_{x}^{M}} d s\right\|_{L_{t}^{M}} \\
& \lesssim C_{0}^{-1} R^{2} \delta^{11 / 4-\delta_{1}}\left\|\langle t\rangle^{1 / 2-\delta_{2}} \int_{0}^{t}(t-s)^{-1 / 2+1 / M}\langle s\rangle^{-3 / 2+\delta_{3}} d s\right\|_{L_{t}^{M}} \lesssim C_{0}^{-1} R^{2} \delta^{11 / 4-\delta_{1}}
\end{aligned}
$$

upon invoking 3.30, 3.29), which in turn can be bounded by $\leq \frac{R}{100} \delta$ upon choosing $\delta$ etc. small enough. Now assume that $t \geq \delta^{-1 / 2}$. Then we get

$$
\begin{aligned}
& C_{0}^{-1} \| \chi_{>\delta^{-1 / 2}}(t)\langle t\rangle^{1 / 2-\delta_{2}} \\
& \cdot \int_{0}^{t} \| e^{i(t-s) \mathcal{H}}\left[\left(\begin{array}{cc}
0 & -e^{2 i\left(\Psi-\Psi_{\infty}\right)_{1}(s)-2 i\left(\Psi-\Psi_{\infty}\right)_{1}(t)}+1 \\
e^{-2 i\left(\Psi-\Psi_{\infty}\right)_{1}(s)+2 i\left(\Psi-\Psi_{\infty}\right)_{1}(t)}-1 & 0
\end{array}\right)\right. \\
& \left.\cdot \phi_{0}^{4}\left(\frac{\tilde{U}^{(t)}(s)}{\tilde{U}^{(t)}(s)}\right)\right]_{\mathrm{dis}}\left\|_{L_{x}^{M}} d s\right\|_{L_{t}^{M}} \\
& \lesssim C_{0}^{-1} R \delta\left\|\chi_{>\delta^{-1 / 2}}(t)\langle t\rangle^{1 / 2-\delta_{2}} \int_{0}^{t}(t-s)^{-1 / 2+1 / M}\langle s\rangle^{-3 / 2+\delta_{3}} d s\right\|_{L_{t}^{M}} \\
& \lesssim C_{0}^{-1} \delta^{-1 / M+\delta_{2} / 2} \delta R,
\end{aligned}
$$


which is also $\leq \frac{R}{100} \delta$ upon choosing $\delta$ small enough. The remaining local terms in $[\ldots]_{\text {dis }}$ can be handled analogously, so we now consider the contribution of the non-local term, which is

$$
C_{0}^{-1}\left\|\langle t\rangle^{1 / 2-\delta_{2}}\right\| \int_{0}^{t} e^{i(t-s) \mathcal{H}}\left(\begin{array}{c}
\left|\tilde{U}^{(t)}\right|^{4} \tilde{U}^{(t)}(s, \cdot) \\
-\left|\tilde{U}^{(t)}\right|^{4} \tilde{U}^{(t)}(s, \cdot)
\end{array}\right) d s\left\|_{L_{x}^{M}}\right\|_{L_{t}^{M}} .
$$

Again referring to 3.30, as well as Theorem 2.1. and using Hölder's inequality, we can bound this by

$$
\begin{aligned}
& \lesssim C_{0}^{-1}\left\|\langle t\rangle^{1 / 2-\delta_{2}}\left(R C_{0}\right)^{5} \delta^{5}\left[\int_{0}^{t}\left[(t-s)^{-1 / 2+1 / M}\langle s\rangle^{-3 / 2+3 \delta_{2}}\right]^{M /(M-5)} d s\right]^{(M-5) / M}\right\|_{L_{t}^{M}} \\
& \lesssim \frac{R}{100} \delta
\end{aligned}
$$

upon choosing $\delta>0$ small enough, as desired. The estimates for $\left\|\langle t\rangle^{-\delta_{2}}\right\| \tilde{U}(t, \cdot)\left\|_{L_{x}^{2}}\right\|_{L_{t}^{M}}$ and $\sup _{\phi \in \mathcal{A}} \sup _{s \geq 0}\langle s\rangle\left\|\phi \tilde{U}_{\text {dis }}^{\prime}(s, y)\right\|_{L_{s}^{M} L_{y}^{M}}$ are carried out similarly and omitted. This then completes the case $k=0$.

We move on to the case $k=1$. We start by showing

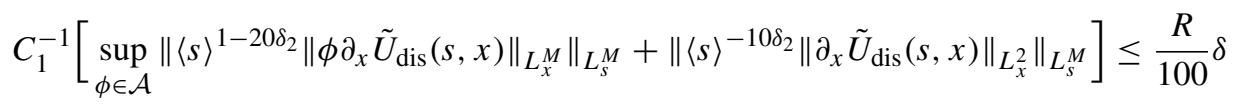

provided $\delta$ is small enough. We use the decomposition

$$
\left.\partial_{x}\left[\left(\begin{array}{c}
\tilde{U} \\
\tilde{\tilde{U}}
\end{array}\right)_{\mathrm{dis}}\right]=\left[\partial_{x}\left[\left(\begin{array}{c}
\tilde{U} \\
\tilde{\tilde{U}}
\end{array}\right)_{\mathrm{dis}}\right]\right]_{\mathrm{dis}}-\sum_{i=1}^{6} /\left(\begin{array}{c}
\tilde{U} \\
\overline{\tilde{U}}
\end{array}\right)_{\mathrm{dis}}, \partial_{x} \xi_{k(i), \text { proper }}\right) \eta_{i, \text { proper }}
$$

The second term here is then estimated using what we know from the case $k=0$, whence we must estimate the first term, which we write as $\left(\frac{\left(\partial_{x} \tilde{U}_{\mathrm{dis}}\right)_{\mathrm{dis}}}{\left(\partial_{x} \tilde{U}_{\mathrm{dis}}\right)_{\mathrm{dis}}}\right)$. Assuming that

$$
\begin{aligned}
B(t):=C_{1}^{-1}\left[\sup _{\phi \in \mathcal{A}}\left\|\langle s\rangle^{1-20 \delta_{2}}\right\|\right. & \phi\left(\partial_{x} \tilde{U}_{\mathrm{dis}}\right)_{\operatorname{dis}}(s, \cdot) \|_{L_{x}^{M} \|_{L_{s}^{M}([0, t])}} \\
& \left.+\left\|\langle s\rangle^{-10 \delta_{2}}\right\|\left(\partial_{x} \tilde{U}_{\mathrm{dis}}\right)_{\operatorname{dis}}(s, \cdot)\left\|_{L_{x}^{2}}\right\|_{L_{s}^{M}([0, t])}\right] \leq R \delta
\end{aligned}
$$

we shall boost this to the bound $\lesssim \frac{R}{100} \delta$. Note that the assumption also gives us the corresponding bounds for $\partial_{x} U$ upon using the already established estimates in the case $k=0$. Commence with the expression

$$
C_{1}^{-1} \sup _{\phi \in \mathcal{A}}\left\|\langle s\rangle^{1-20 \delta_{2}}\right\| \phi\left(\partial_{x} \tilde{U}_{\mathrm{dis}}\right)_{\mathrm{dis}}(s, x) \|_{L_{x}^{M} \|_{L_{s}^{M}([0, t])}} .
$$

If we project (1.8) onto its dispersive part, differentiate with respect to $x$, and then project again onto the dispersive part, we produce additional local source terms of the schematic forms $V U,\langle V, U\rangle W$ for certain Schwartz functions $V, W$, in addition to terms involving 
$\partial_{x} U$. The former terms are handled by using the already established estimates for $k=0$ as well as the assumption $C_{1} \gg C_{0}$. As for the latter, the most difficult local term leads to the Duhamel term 45

$$
\begin{aligned}
& C_{1}^{-1} \|\langle s\rangle^{1-20 \delta_{2}} \\
& \int_{0}^{s} \| \phi(x) e^{i(s-\lambda) \mathcal{H}}\left[\left(\begin{array}{cc}
0 & -e^{2 i\left(\Psi-\Psi_{\infty}\right)_{1}(\lambda)-2 i\left(\Psi-\Psi_{\infty}\right)_{1}(s)}+1 \\
e^{-2 i\left(\Psi-\Psi_{\infty}\right)_{1}(\lambda)+2 i\left(\Psi-\Psi_{\infty}\right)_{1}(s)}-1 & 0
\end{array}\right)\right. \\
& \left.\cdot \phi_{0}^{4}\left(\frac{\partial_{x} \tilde{U}^{(s)}(\lambda)}{\partial_{x} \tilde{U}^{(s)}(\lambda)}\right)\right]_{\mathrm{dis}}\left\|_{L_{x}^{M}} d \lambda\right\|_{L_{s}^{M}([0, t])}
\end{aligned}
$$

First restrict integration to the interval $\left[0, s-\delta^{-1 / 2}\langle s\rangle^{6 / M}\right]$. This we can estimate crudely by 46

$$
\begin{aligned}
& \lesssim \Lambda \delta C_{1}^{-1}\left\|\langle s\rangle^{1-20 \delta_{2}+1 / M} \int_{0}^{s-\delta^{-1 / 2}\langle s\rangle^{6 / M}}\langle s\rangle^{2 / M}\langle s-\lambda\rangle^{-3 / 2}\langle\lambda\rangle^{-1+20 \delta_{2}} d \lambda\right\|_{L_{s}^{M}([0, t])} \\
& \leq \frac{R}{100} \delta
\end{aligned}
$$

upon choosing $\delta$ small enough.

On the interval $\left[s-\delta^{-1 / 2}\langle s\rangle^{6 / M}, s\right]$, one proceeds similarly, but exploits the fact that thanks to the proof of Lemma 5.3, we have

$$
\sup _{\lambda \in\left[s-\delta^{-1 / 2}\langle s\rangle^{6 / M}, s\right]}\left|-e^{2 i\left(\Psi-\Psi_{\infty}\right)_{1}(\lambda)-2 i\left(\Psi-\Psi_{\infty}\right)_{1}(s)}+1\right| \lesssim \delta^{3 / 2}\langle s\rangle^{-1 / 2+\delta_{1}+6 / M} .
$$

The remaining local terms involving $\partial_{x} \tilde{U}^{(t)}$ are easier and estimated similarly. Next, consider the non-local term. We have to estimate

$$
C_{1}^{-1}\left\|\langle s\rangle^{1-20 \delta_{2}}\right\| \phi \int_{0}^{s} e^{i(s-\lambda) \mathcal{H}}\left(\begin{array}{c}
\left|\tilde{U}^{(s)}(\lambda, \cdot)\right|^{4} \partial_{x} \tilde{U}^{(s)}(\lambda, \cdot) \\
-\left|\tilde{U}^{(s)}(\lambda, \cdot)\right|^{4} \partial_{x} \tilde{U}^{(s)}(\lambda, \cdot)
\end{array}\right)_{\mathrm{dis}} d \lambda\left\|_{L_{x}^{M}}\right\|_{L_{s}^{M}([0, t])}
$$

in addition to similar terms (Leibniz rule). If we invoke the assumption on $B(t)$, the weighted estimates in Theorem 2.1 as well as the estimates for the case $k=0$ we can

45 One uses the auxiliary variable

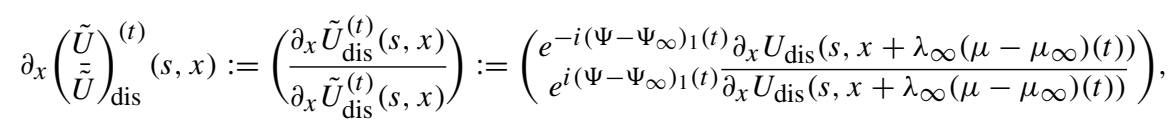

analogously to $\left(\frac{\tilde{U}^{(t)}(s, \cdot)}{\tilde{U}^{(t)}(s, \cdot)}\right)$ which we used before. We similarly introduce $\partial_{x}\left(\frac{\tilde{U}}{\tilde{U}}\right)^{(t)}:=\left(\frac{\partial_{x} \tilde{U}^{(t)}}{\partial_{x} \tilde{U}^{(t)}}\right)$. 46 One again uses Hölder's inequality to handle the fact that we only control $\left\|\phi \partial_{x} \tilde{U}^{(s)}(\lambda, x)\right\|_{L_{\lambda}^{M} L_{x}^{M}}$. 
bound this by

$$
\begin{aligned}
& \lesssim R^{5} C_{0}^{4} \delta^{5}\left\|\langle s\rangle^{1-20 \delta_{2}} \int_{0}^{s}(s-\lambda)^{-1+20 \delta_{2}-1 / M}\langle\lambda\rangle^{4 \delta_{2}+10 \delta_{2}-3 / 2}\langle\lambda\rangle^{1 / 2-20 \delta_{2}+6 / M} d \lambda\right\|_{L_{s}^{M}([0, t])} \\
& \lesssim \frac{R}{100} \delta .
\end{aligned}
$$

We move on to control $\left\|\langle s\rangle^{-10 \delta_{2}}\right\|\left(\partial_{x} \tilde{U}_{\mathrm{dis}}\right)_{\mathrm{dis}}(s, x)\left\|_{L_{x}^{2}}\right\|_{L_{s}^{M}([0, t])}$. Note that we cannot simply reiterate the Duhamel procedure here, since this would lead to a loss for the local terms ${ }^{47}$ Observe that (1.8) implies the following schematic relation:

$$
\begin{aligned}
i \partial_{s}\left[\partial_{x} \tilde{U}_{\mathrm{dis}}^{(t)}\right] & (s, \cdot)+\partial_{x}^{2}\left[\partial_{x} \tilde{U}_{\mathrm{dis}}^{(t)}\right](s, \cdot)-\partial_{x} \tilde{U}_{\mathrm{dis}}^{(t)}(s, \cdot) \\
& =\left[V \partial_{x} \tilde{U}^{(t)}\right](s, \cdot)+\left[V \overline{\partial_{x} \tilde{U}^{(t)}}\right](s, \cdot)+\cdots+\left[\left|\tilde{U}^{(t)}\right|^{4} \partial_{x} \tilde{U}^{(t)}\right](s, \cdot),
\end{aligned}
$$

where the subscripts really refer to the top entry of the dispersive part of the corresponding vector-valued function. As usual $V$ refers to certain Schwartz functions which may depend on $t$ and $s$. We deduce that

$$
\begin{aligned}
& i \partial_{\lambda} \int_{-\infty}^{\infty}\left|\partial_{x} \tilde{U}_{\mathrm{dis}}^{(s)}\right|^{2}(\lambda, \cdot) d x=2 \Im \int_{-\infty}^{\infty} i \partial_{\lambda}\left[e^{i \lambda} \partial_{x} \tilde{U}_{\mathrm{dis}}^{(s)}\right](\lambda, \cdot) \overline{e^{i \lambda} \partial_{x} \tilde{U}_{\mathrm{dis}}^{(s)}(\lambda, \cdot)} d x \\
& =2 \Im \int_{-\infty}^{\infty}\left[V \partial_{x} \tilde{U}_{\mathrm{dis}}^{(s)}(\lambda, \cdot) \overline{\partial_{x} \tilde{U}_{\mathrm{dis}}^{(s)}(\lambda, \cdot)}+\cdots+\left|\tilde{U}^{(s)}\right|^{4} \partial_{x} \tilde{U}^{(s)} \overline{\partial_{x} \tilde{U}^{(s)}}(\lambda, \cdot)\right] d x .
\end{aligned}
$$

Using the already improved local bound, we can estimate

$$
\begin{array}{r}
\left\|\langle s\rangle^{-20 \delta_{2}} \int_{0}^{s}\left|\Im \int_{-\infty}^{\infty} V \partial_{x} \tilde{U}^{(s)}(\lambda, \cdot) \overline{\partial_{x} \tilde{U}^{(s)}(\lambda, \cdot)} d x\right| d \lambda\right\|_{L_{s}^{M}([0, t])} \\
\lesssim\left(\frac{C_{1} R}{100}\right)^{2} \delta^{2} \int_{0}^{s}\langle\lambda\rangle^{-2\left(1-20 \delta_{2}\right)} d \lambda
\end{array}
$$

which is integrable in $\lambda$ upon choosing $\delta_{2}$ small enough. The remaining expressions on the right hand side of (5.2) can be estimated similarly, and combining the preceding estimates we get the improved bound on $B(t)$, as desired.

Next, consider the norm $\left\|\langle s\rangle^{1 / 2-25^{k} \delta_{2}}\right\|\left(\partial_{x} \tilde{U}_{\text {dis }}\right)_{\text {dis }}(s, \cdot)\left\|_{L_{x}^{M}}\right\|_{L_{s}^{M}([0, T))}$. This we estimate by reverting to the usual Duhamel's formula; we treat again first the local term of least time decay, as the others can be treated similarly and are simpler: we have

\footnotetext{
47 More precisely, it appears that the extra factor $\langle s\rangle^{-10 \delta_{2}}$ should allow one to gain a bit; however, the loss from the weak local decay control, i.e., $\left\|\phi \partial_{x} U(s, \cdot)\right\|_{L_{x}^{\infty}} \lesssim\langle s\rangle^{-1+20 \delta_{2}}$, is too much. On the other hand, if one strengthened the weight in the $L^{2}$-norm to $\langle s\rangle^{-(20+) \delta_{2}}$, one would encounter difficulties in retrieving the weak local estimate. The reader may ask why we do not build in the strong local decay for all higher derivatives to begin with. The problem with this is that we would have to gain control over more and more derivatives that way, indeed forcing control over infinitely many derivatives.
} 


$$
\begin{aligned}
& \left\|\langle s\rangle^{1 / 2-25 \delta_{2}}\right\| \int_{0}^{s} e^{i(s-\lambda) \mathcal{H}}\left[\left(-e^{-2 i\left(\Psi-\Psi_{\infty}\right)_{1}(\lambda)+2 i\left(\Psi-\Psi_{\infty}\right)_{1}(s)}+1\right) V \partial_{x} U\right]_{\mathrm{dis}}(\lambda, \cdot) d \lambda\left\|_{L_{x}^{M}}\right\|_{L_{s}^{M}} \\
& \lesssim \frac{R C_{1}}{100} \delta\left\|\langle s\rangle^{1 / 2-25 \delta_{2}} \int_{0}^{s}(s-\lambda)^{-1 / 2+1 / M} \lambda^{-1+20 \delta_{2}} d \lambda\right\|_{L_{s}^{M}} .
\end{aligned}
$$

This is easily seen to also improve the bound $R \delta C_{1}$, if necessary by improving the preceding estimates for $B(t)$. Proceeding to higher derivatives $k \geq 2$ is an elementary induction, recycling the same estimates. Observe that if one differentiates the quintilinear non-local term $k$ times, one obtains schematically either $\left|\tilde{U}^{(s)}\right|^{4} \partial_{x}^{k}\left(\tilde{U}^{(s)}\right)$, or else $\partial_{x}^{k-1}\left(\tilde{U}^{(s)}\right) \partial_{x}\left(\tilde{U}^{(s)}\right)\left(\tilde{U}^{(s)}\right)^{2} \overline{\tilde{U}^{(s)}}$ or else $\left.\partial_{x}^{\alpha_{1}}\left(\tilde{U}^{(s)}\right) \partial_{x}^{\alpha_{2}} \overline{\tilde{U}^{(s)}}\right) \ldots \partial_{x}^{\alpha_{5}}\left(\tilde{U}^{(s)}\right)$, where all $\alpha_{i}<$ $k-1$, or terms equivalent to these to all intents and purposes. The first term in this list can be treated just as before, using the estimates for $U$. For the second, one estimates

$$
\begin{gathered}
\left\|\left(\partial_{x}^{k-1}\left(\tilde{U}^{(s)}\right) \partial_{x}\left(\tilde{U}^{(s)}\right)\left(\tilde{U}^{(s)}\right)^{2} \overline{\tilde{U}^{(s)}}\right)(\lambda, \cdot)\right\|_{L_{x}^{M^{\prime}}} \leq R^{5} C_{k-1} C_{1} C_{0}^{3} \delta^{5} \lambda^{10^{k-1} \delta_{2}+10 \delta_{2}+3 \delta_{2}}\langle\lambda\rangle^{-3 / 2} \\
\cdot\left\|\langle\lambda\rangle^{1 / 2-\delta_{2}} \tilde{U}^{(s)}(\lambda, \cdot)\right\|_{L_{x}^{M}}\left\|\langle\lambda\rangle^{-10^{k-1} \delta_{2}} \partial_{x}^{k-1} \tilde{U}^{(s)}(\lambda, \cdot)\right\|_{L_{x}^{2+}}^{2+}\left\|\langle\lambda\rangle^{-\delta_{2}} \partial_{x} \tilde{U}^{(s)}(\lambda, \cdot)\right\|_{L_{x}^{2}},
\end{gathered}
$$

and we have

$$
\lambda^{10^{k-1} \delta_{2}+10 \delta_{2}+3 \delta_{2}}\langle\lambda\rangle^{-3 / 2} \lesssim\langle\lambda\rangle^{-3 / 2+15^{k} \delta_{2}} .
$$

Thus one can comfortably absorb an extra weight $\lambda^{1 / 2-20^{k} \delta_{2}+4 / M}$ here, and continue as before. The estimate for the last term in the above list is similar. Time derivatives can now be handled upon turning them into spatial derivatives via (1.8).

Lemma 5.6. Under the same hypotheses as in the previous lemma, we have the inequality

$$
\sum_{1 \leq k \leq N-1} K^{-k} \sup _{3 i+j=k}\left\|C \partial_{s}^{i} \partial_{y}^{j} U^{\prime}(s, \cdot)\right\|_{L_{s}^{M} L_{y}^{2}} \leq R \delta,
$$

where $K=\langle T\rangle^{100}$.

Proof. This is a consequence of the fact that the operators $y-2 p s$ and $i \partial_{s}+\Delta$ commute, as well as using the already established estimates from the previous lemma and crude bounds.

We now turn to the much more difficult estimates involving the local decay of $U$, as well as the conservation of $\|C U\|_{L_{y}^{2}}$. This is where we have to penetrate more deeply into the iteration in order to be able to close the estimates. The proof of the next lemma is long and hard and ends on page 74, we shall refer to this estimate as the Strong Local Dispersive Estimate or SLDE:

Lemma 5.7. Let $\Gamma_{T}^{\prime} \in A_{[0, T)}^{(2)}$. Then provided the conditions of Theorem 4.5 are satisfied,

$$
\sup _{\phi \in \mathcal{A}} \sup _{T \geq s \geq 0}\left\|\langle s\rangle^{3 / 2-\delta_{3}} \phi U^{\prime}(s, \cdot)\right\|_{L_{y}^{\infty}} \leq \frac{R}{2} \delta .
$$

Proof. We may assume that $\Gamma_{T}^{\prime}=T_{A, \lambda, \beta, \omega, \gamma, \mu} \Gamma_{T}, \Gamma_{T} \in A_{[0, T)}^{(1)}$. In particular, we may assume that $\Gamma_{T}$ is a convex linear combination of elements in the image of $T_{A, \lambda, \beta, \omega, \gamma, \mu}$ 
applied to $A_{[0, T)}^{(0)}$ and satisfying all the estimates implied in the definition of $A_{[0, T)}^{(0)}$. For this we need to employ (3.27), which forces us to distinguish between the different kinds of expressions on the right hand side. Clearly we may assume $t \geq 1$. We first observe that Theorem 2.1 in conjunction with our assumptions on $A(\cdot)$ as well as 3.14, 3.29, 3.30) and Lemma 5.3 imply that the free contribution is acceptable, with a $t^{\delta_{3}}$ to spare:

$$
\begin{aligned}
\sup _{0 \leq t}\langle t\rangle^{3 / 2} \| e^{i t \mathcal{H}} P_{S}\left[( \begin{array} { c c } 
{ e ^ { i ( \Psi _ { \infty } - \Psi ) _ { 1 } ( t ) } } & { 0 } \\
{ 0 } & { e ^ { - i ( \Psi _ { \infty } - \Psi ) _ { 1 } ( t ) } }
\end{array} ) \left[\left(\begin{array}{c}
A\left(\cdot+\lambda_{\infty}\left(\mu-\mu_{\infty}\right)(t)\right) \\
\bar{A}\left(\cdot+\lambda_{\infty}\left(\mu-\mu_{\infty}\right)(t)\right)
\end{array}\right)_{\mathrm{dis}}\right.\right. \\
\\
\left.\left.+\sum_{j=1}^{6} \alpha_{j} \eta_{j, \operatorname{proper}}\left(\cdot+\lambda_{\infty}\left(\mu-\mu_{\infty}\right)(t)\right)\right]\right] \| \leq \frac{R}{100} \delta,
\end{aligned}
$$

by choosing $R$ large enough. We next subdivide $[\ldots]_{\text {dis }}$ in the integrand of the Duhamel term in (3.27) into local and non-local contributions. As for the local contributions, the one with least temporal decay and hence most delicate is (written schematically)

$$
\int_{0}^{t} e^{i(t-s) \mathcal{H}}\left[-e^{-2 i\left(\Psi-\Psi_{\infty}\right)_{1}(s)+2 i\left(\Psi-\Psi_{\infty}\right)_{1}(t)}+1\right]\left[V \tilde{U}^{(t)}\right]_{\mathrm{dis}}(s, \cdot) d s .
$$

One estimates (using Theorem 2.1)

$$
\begin{array}{r}
\langle t\rangle^{3 / 2-\delta_{3}}\left\|\phi(x) \int_{0}^{t-\delta^{-1 / 2}} e^{i(t-s) \mathcal{H}}\left[-e^{-2 i\left(\Psi-\Psi_{\infty}\right)_{1}(s)+2 i\left(\Psi-\Psi_{\infty}\right)_{1}(t)}+1\right]\left[V \tilde{U}^{(t)}\right]_{\operatorname{dis}}(s, \cdot) d s\right\|_{L_{x}^{\infty}} \\
\lesssim\langle t\rangle^{3 / 2-\delta_{3}} R \delta \int_{0}^{t-\delta^{-1 / 2}}\langle t-s\rangle^{-3 / 2}\langle s\rangle^{-3 / 2+\delta_{3}} d s \lesssim R \delta^{1+\delta_{3} / 2},
\end{array}
$$

which leads to the bound $\lesssim \frac{R}{100} \delta$ upon choosing $\delta>0$ small enough. Moreover, using Lemma 5.3 , one gets the same estimate for the integral over $\left[t-\delta^{-1 / 2}, t\right]$, as desired. The remaining local terms in $[\ldots]_{\text {dis }}$ can be handled similarly. Observe that up to this point we still have not invoked the iterated structure of $A_{[0, T)}^{(1)}$. We shall do so in the course of the ensuing considerations, clearly indicating where the extra iterated structure is needed. Note that by definition, we may write

$$
\left(\begin{array}{c}
\tilde{U} \\
\overline{\tilde{U}}
\end{array}\right)_{\mathrm{dis}}=\sum_{i=1}^{k} \alpha_{i}\left(\begin{array}{c}
\tilde{U}_{i} \\
\overline{\tilde{U}}_{i}
\end{array}\right)_{\mathrm{dis}}
$$

where $\sum_{i} \alpha_{i}=1$ with $\alpha_{i} \geq 0$ for all $i$. The same comment applies to the modulation variables $\nu_{1}$ etc., as well as the root parameters $\lambda_{i}$. In particular, we can write

$$
\begin{aligned}
\left(\begin{array}{c}
\tilde{U}^{(t)}(s, \cdot) \\
\tilde{U}^{(t)}(s, \cdot)
\end{array}\right)= & \left(\begin{array}{cc}
e^{i\left(\Psi-\Psi_{\infty}\right)_{1}(s)-i\left(\Psi-\Psi_{\infty}\right)_{1}(t)} & 0 \\
0 & e^{-i\left(\Psi-\Psi_{\infty}\right)_{1}(s)+i\left(\Psi-\Psi_{\infty}\right)_{1}(t)}
\end{array}\right) \\
& \cdot\left(\begin{array}{c}
\tilde{U}\left(s, \cdot-\lambda_{\infty}\left(\mu-\mu_{\infty}\right)\right) \\
\tilde{\tilde{U}}\left(s, \cdot-\lambda_{\infty}\left(\mu-\mu_{\infty}\right)\right)
\end{array}\right) .
\end{aligned}
$$

Here, according to the construction defined in the iterative step at the beginning of this section, the functions $\left(\Psi-\Psi_{\infty}\right)_{1}, \lambda_{\infty}\left(\mu-\mu_{\infty}\right)$ are obtained from the variables $v_{1}$ etc. 
by the steps described there; the variables $v_{1}$ etc. are convex linear combinations of the variables $v_{1, i}$ etc. Furthermore, we have

$$
\left(\begin{array}{c}
\tilde{U} \\
\tilde{U}
\end{array}\right)=\sum_{i} \alpha_{i}\left[\left(\begin{array}{c}
\tilde{U}_{i} \\
\tilde{\tilde{U}}_{i}
\end{array}\right)_{\mathrm{dis}}+\sum_{k=1}^{6} \lambda_{k, i} \eta_{k, \text { proper }}\right], \quad \alpha_{i} \geq 0 \forall i, \quad \sum_{i} \alpha_{i}=1
$$

We shall use this decomposition at one step below in the proof of the lemma. For the most part, though, it will suffice to know that $\Gamma_{T} \in A_{[0, T)}^{(0)}$. We now turn to the more difficult task of dealing with the non-local term, i.e., the expression

$$
\langle t\rangle^{3 / 2-\delta_{3}}\left\|\phi(x) \int_{0}^{t} e^{i(t-s) \mathcal{H}}\left(\begin{array}{c}
\left|\tilde{U}^{(t)}\right|^{4} \tilde{U}^{(t)}(s, \cdot) \\
-\left|\tilde{U}^{(t)}\right|^{4} \tilde{U}^{(t)}(s, \cdot)
\end{array}\right)_{\mathrm{dis}} d s\right\|_{L_{x}^{\infty}} .
$$

We intend to turn this into an expression of the following form:

$$
\langle t\rangle^{3 / 2-\delta_{3}}\left\langle\int_{0}^{t} e^{i(t-s) \mathcal{H}}\left(\begin{array}{c}
\left|\tilde{U}^{(t)}\right|^{4} \tilde{U}^{(t)}(s, \cdot) \\
-\left|\tilde{U}^{(t)}\right|^{4} \overline{\tilde{U}^{(t)}}(s, \cdot)
\end{array}\right)_{\mathrm{dis}} d s,\left(\begin{array}{c}
\phi \\
\psi
\end{array}\right)\right\rangle,
$$

for suitable Schwartz functions $\phi, \psi$. The device for achieving this is the discrete Fourier transform. First, using a partition of unity $\left\{\phi_{i}\right\}$ subordinate to intervals of length $2 \pi$, we reduce to estimating

$$
\phi_{j}(x) \phi(x) \int_{0}^{t} e^{i(t-s) \mathcal{H}}\left(\begin{array}{c}
\left|\tilde{U}^{(t)}\right|^{4} \tilde{U}^{(t)}(s, \cdot) \\
-\left|\tilde{U}^{(t)}\right|^{4} \tilde{\tilde{U}}^{(t)}(s, \cdot)
\end{array}\right)_{\mathrm{dis}} d s
$$

Write

$$
i \phi \phi_{j} \int_{0}^{t} e^{i(t-s) \mathcal{H}}\left(\begin{array}{c}
\left|\tilde{U}^{(t)}\right|^{4} \tilde{U}^{(t)}(s) \\
-\left|\tilde{U}^{(t)}\right|^{4} \tilde{U}^{(t)}(s)
\end{array}\right)_{\mathrm{dis}} d s=\sum_{n \in \mathbb{Z}}\left(\begin{array}{c}
a_{n j} e^{i n\left(x-x_{j}\right)} \\
\bar{a}_{n j} e^{-i n\left(x-x_{j}\right)}
\end{array}\right) .
$$

We have

$$
\begin{aligned}
& \Re a_{n j}=\frac{i}{4 \pi}\left\langle\phi \phi_{j} \int_{0}^{t} e^{i(t-s) \mathcal{H}}\left(\begin{array}{c}
\left|\tilde{U}^{(t)}\right|^{4} \tilde{U}^{(t)}(s) \\
-\left|\tilde{U}^{(t)}\right|^{4} \tilde{U}^{(t)}(s)
\end{array}\right)_{\mathrm{dis}} d s,\left(\begin{array}{c}
e^{i n\left(x-x_{j}\right)} \\
e^{-i n\left(x-x_{j}\right)}
\end{array}\right)\right\rangle, \\
& \Im a_{n j}=\frac{1}{4 \pi}\left\langle\phi \phi_{j} \int_{0}^{t} e^{i(t-s) \mathcal{H}}\left(\begin{array}{c}
\left|\tilde{U}^{(t)}\right|^{4} \tilde{U}^{(t)}(s) \\
-\left|\tilde{U}^{(t)}\right|^{4} \tilde{U}^{(t)}(s)
\end{array}\right)_{\mathrm{dis}} d s,\left(\begin{array}{c}
e^{i n\left(x-x_{j}\right)} \\
-e^{-i n\left(x-x_{j}\right)}
\end{array}\right)\right\rangle .
\end{aligned}
$$

We can rearrange the first of these expressions as follows:

$$
\Re a_{n j}=\frac{i}{4 \pi}\left\langle\int_{0}^{t} e^{i(t-s) \mathcal{H}}\left(\begin{array}{c}
\left|\tilde{U}^{(t)}\right|^{4} \tilde{U}^{(t)}(s) \\
-\left|\tilde{U}^{(t)}\right|^{4} \tilde{U}^{(t)}(s)
\end{array}\right)_{\mathrm{dis}} d s,\left(\begin{array}{c}
\phi \phi_{j} e^{i n\left(x-x_{j}\right)} \\
\phi \phi_{j} e^{-i n\left(x-x_{j}\right)}
\end{array}\right)\right\rangle,
$$

and similarly for the imaginary part. We now claim that we only need to focus on relatively small values on $n$, specifically $|n|\left\langle\delta^{-\epsilon}\langle t\rangle^{\epsilon}\right.$ for small $\epsilon>\epsilon_{0}(N)$. Indeed, note that

$$
\left\langle\left(\frac{\tilde{U}^{(t)}}{\tilde{U}^{(t)}}\right), \phi e^{i n x}\right\rangle=\frac{1}{(i n)^{N}}\left\langle\partial_{x}^{N}\left[\phi\left(\frac{\tilde{U}^{(t)}}{\tilde{U}^{(t)}}\right)\right], e^{i n x}\right\rangle \lesssim \delta^{2} R\langle t\rangle^{-3 / 2}
$$


for suitable choice of $\epsilon$, and $|n| \geq \delta^{-\epsilon}\langle t\rangle^{\epsilon}$. We shall simplify the term $\phi \phi_{j} e^{-i n\left(x-x_{j}\right)}$ to $\phi$, while taking account that we need to pay a factor $\delta^{-\epsilon}\langle t\rangle^{\epsilon}$ at the end for summing over $n$. We can easily absorb this error, since we may assume $\delta_{3} \gg \epsilon$. We now need to estimate the following expression:

$$
\int_{0}^{t}\left\langle\left(\begin{array}{c}
\left|\tilde{U}^{(t)}\right|^{4} \tilde{U}^{(t)}(s) \\
-\left|\tilde{U}^{(t)}\right|^{4} \tilde{U}^{(t)}(s)
\end{array}\right), e^{-i(t-s) \mathcal{H}^{*}}\left(\begin{array}{c}
\phi \\
\psi
\end{array}\right)_{\mathrm{dis}}\right\rangle d s .
$$

We distinguish between the cases $s>t / 2, s \leq t / 2$, which aside from a simple technicality are treated by the same method. We shall use the notation $P_{\leq a}, P_{a}, P_{>a}, a \in \mathbb{R}_{>0}$ dyadic ${ }^{48}$ for the standard Littlewood-Paley multipliers (see e.g. [ $[\overline{\mathrm{St}}]$ ). We shall also assume that $\tilde{U}$ (and hence also $\tilde{U}^{(t)}$ ) satisfies pointwise-in-time estimates below in order to simplify the exposition; thus we shall assume bounds of the form $\langle s\rangle^{1 / 2-\delta_{2}}\|\tilde{U}(s, \cdot)\|_{L_{x}^{\infty} \leq}$ $\delta R$ etc. It will be straightforward to adjust the arguments below to the case of weightedin-time norms, since we assume $M \gg \delta_{2}^{-1}$ and hence we can absorb losses of order $\langle s\rangle^{O(1 / M)}$.

Case A: $s<t / 2$. The idea is to exploit the pseudo-conformal operator to reduce at least one of the two $|U|^{2}$ 's to frequency $<s^{-3 / 4}$. In this case, one exploits the fact that the distorted Fourier transform vanishes at the origin. We start by chopping things apart, by specializing to the following two terms:

$$
\begin{aligned}
& \int_{0}^{t / 2}\left\langle\left(\begin{array}{c}
\chi_{>0}\left|\tilde{U}^{(t)}\right|{ }^{4} \tilde{U}^{(t)}(s) \\
-\chi_{>0}\left|\tilde{U}^{(t)}\right|{ }^{4} \tilde{U}^{(t)}(s)
\end{array}\right), e^{-i(t-s) \mathcal{H}^{*}}\left(\begin{array}{c}
\phi \\
\psi
\end{array}\right)_{\mathrm{dis}}\right\rangle d s, \\
& \int_{0}^{t / 2}\left\langle\left(\begin{array}{c}
\chi_{<0}\left|\tilde{U}^{(t)}\right|{ }^{4} \tilde{U}^{(t)}(s) \\
-\chi_{<0}\left|\tilde{U}^{(t)}\right|{ }^{4} \tilde{\tilde{U}}^{(t)}(s)
\end{array}\right), e^{-i(t-s) \mathcal{H}^{*}}\left(\begin{array}{c}
\phi \\
\psi
\end{array}\right)_{\mathrm{dis}}\right\rangle d s,
\end{aligned}
$$

where $\chi>0$ is the Heaviside function localizing to $x>0$. Both are treated the same way, so consider the first expression: rewrite it as

$$
\int_{0}^{t / 2}\left\langle\left(\begin{array}{c}
\chi>0\left|\tilde{U}^{(t)}\right|^{4}(s) \\
-\chi>0\left|\tilde{U}^{(t)}\right|^{4}(s)
\end{array}\right),\left(\begin{array}{c}
\overline{\tilde{U}^{(t)}}(s) \\
\tilde{U}^{(t)}(s)
\end{array}\right) \times e^{-i(t-s) \mathcal{H}^{*}}\left(\begin{array}{c}
\phi \\
\psi
\end{array}\right)_{\mathrm{dis}}\right\rangle d s,
$$

where $\times$ denotes componentwise multiplication 49

We commence by reducing $\left(\begin{array}{c}\chi>0\left|\tilde{U}^{(t)}\right|^{4}(s) \\ -\chi_{>0}\left|\tilde{U}^{(t)}\right|^{4}(s)\end{array}\right)$ to its dispersive part. To achieve this, note that

$$
\begin{aligned}
& \left(\begin{array}{c}
\chi_{>0}\left|\tilde{U}^{(t)}\right|^{4}(s) \\
-\chi_{>0}\left|\tilde{U}^{(t)}\right|^{4}(s)
\end{array}\right)=\left(\begin{array}{c}
\chi_{>0}\left|\tilde{U}^{(t)}\right|^{4}(s) \\
-\chi_{>0}\left|\tilde{U}^{(t)}\right|^{4}(s)
\end{array}\right)_{\mathrm{dis}} \\
& +\sum_{j=1}^{6} a_{k(j)}\left\langle\left(\begin{array}{c}
\chi>0\left|\tilde{U}^{(t)}\right|^{4}(s) \\
-\chi>0\left|\tilde{U}^{(t)}\right|^{4}(s)
\end{array}\right), \xi_{k(j), \text { proper }}\right\rangle \eta_{j, \text { proper }}
\end{aligned}
$$

\footnotetext{
48 We shall more generally mean $P_{\alpha}$ for $\alpha \in \mathbb{R}_{>0}$ to denote $P_{j}$ if $2^{j-1} \leq \alpha<2^{j}$.

49 Also observe that we use the subscript "dis" both with reference to $\mathcal{H}$ as well as $\mathcal{H}^{*}$.
} 
where $\xi_{j \text {,proper }}$ is the basis for the generalized root space of $\mathcal{H}^{*}$, while $\eta_{j \text {,proper }}$ is the basis for the generalized root space of $\mathcal{H}$, as explained earlier. The $a_{k(j)}$ are suitable numerical coefficients. Then observe that by the improved local dispersive estimate, we have $\left(\epsilon=\epsilon\left(\delta_{3}\right)\right)$

$$
\left|\left\langle\left(\begin{array}{c}
\chi_{>0}\left|\tilde{U}^{(t)}\right|^{4}(s) \\
-\chi_{>0}\left|\tilde{U}^{(t)}\right|^{4}(s)
\end{array}\right), \xi_{j, \text { proper }}\right\rangle\right| \lesssim\left(R C_{0}\right)^{4} \delta^{4}\langle s\rangle^{-6+\epsilon},
$$

whence we treat the contribution of this part to the above integral expression by

$$
\lesssim\left(R C_{0}\right)^{4} \delta^{4} \int_{0}^{t / 2}(t-s)^{-3 / 2}\langle s\rangle^{-6+\epsilon} d s,
$$

which is better than what we need. Thus we now consider

$$
\int_{0}^{t / 2}\left\langle\left(\begin{array}{c}
\chi_{>0}\left|\tilde{U}^{(t)}\right|^{4}(s) \\
-\chi_{>0}\left|\tilde{U}^{(t)}\right|^{4}(s)
\end{array}\right)_{\mathrm{dis}},\left(\begin{array}{c}
\overline{\tilde{U}^{(t)}}(s) \\
\tilde{U}^{(t)}(s)
\end{array}\right) \times e^{-i(t-s) \mathcal{H}^{*}}\left(\begin{array}{c}
\phi \\
\psi
\end{array}\right)_{\mathrm{dis}}\right\rangle d s
$$

Using the distorted Plancherel's Theorem 2.3 we can equate this with

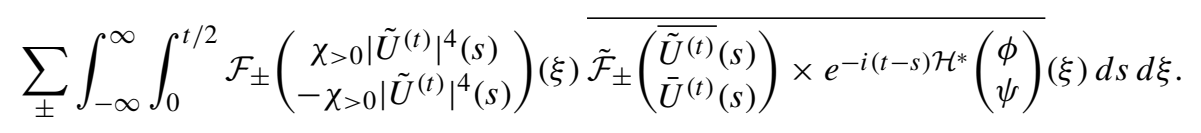

Observe that $\mathcal{F}_{ \pm}(\phi)(\xi)=\left\langle\phi, \sigma_{3} e_{ \pm}(x, \xi)\right\rangle$ and $\tilde{\mathcal{F}}(\phi)(\xi)=\left\langle\phi, e_{ \pm}(x, \xi)\right\rangle$. The cases \pm are treated exactly analogously, so we stick with the + case. Break the $\xi$-integral into two parts, over $[0, \infty)$ and over $(-\infty, 0]$. Commence with the case $\xi \in[0, \infty)$. Write

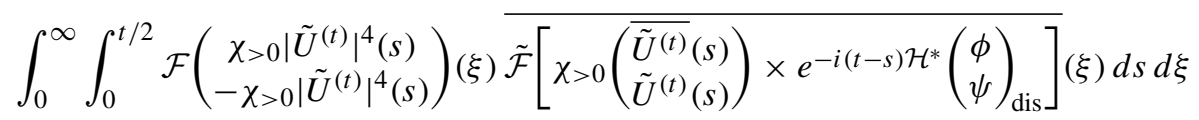

$$
\begin{aligned}
& =\int_{0}^{\infty} \int_{0}^{t / 2}\left\langle\left(\begin{array}{c}
\chi>0\left|\tilde{U}^{(t)}\right|^{4}(s) \\
-\chi>0 \\
-\left.\tilde{U}^{(t)}\right|^{4}(s)
\end{array}\right), s(\xi) e^{i x \xi} \underline{e}+\phi(x, \xi)\right\rangle \\
& \overline{\tilde{\mathcal{F}}\left[\chi>0\left(\begin{array}{l}
\overline{\tilde{U}^{(t)}}(s) \\
\tilde{U}^{(t)}(s)
\end{array}\right) \times e^{-i(t-s) \mathcal{H}^{*}}\left(\begin{array}{l}
\phi \\
\psi
\end{array}\right)_{\mathrm{dis}}\right]}(\xi) d s d \xi
\end{aligned}
$$

recalling Theorem 2.2. We first treat the simple contribution coming from the rapidly decaying function $\phi(x, \xi)$. As before, observe that

$$
\left|\left\langle\left(\begin{array}{l}
\chi_{>0}\left|\tilde{U}^{(t)}\right|^{4}(s) \\
\chi_{>0}\left|\tilde{U}^{(t)}\right|^{4}(s)
\end{array}\right), \phi(x, \xi)\right\rangle\right| \lesssim\langle s\rangle^{-6+\epsilon}, \quad \epsilon=\epsilon\left(\delta_{3}\right) .
$$

Indeed, we can estimate the $L_{\xi}^{2}$-norm of the function on the left in this fashion. Moreover, we have

$$
\left\|\tilde{\mathcal{F}}\left[\chi>0\left(\begin{array}{c}
\bar{U}(s) \\
U(s)
\end{array}\right) \times e^{-i(t-s) \mathcal{H}^{*}}\left(\begin{array}{c}
\phi \\
\psi
\end{array}\right)_{\mathrm{dis}}\right](\xi)\right\|_{L_{\xi}^{2}} \lesssim\langle t-s\rangle^{-3 / 2} s^{1+\epsilon\left(\delta_{2}\right)} .
$$


We are using here the pseudo-conformal almost conservation, which is part of our assumptions 3.30:

$$
\|(x-2 s p) \tilde{U}(s, \cdot)\|_{L_{x}^{2}} \lesssim R \delta, \quad p=-i \frac{\partial}{\partial x}
$$

From this we obtain

$$
\begin{aligned}
& \mid \int_{0}^{\infty} \int_{0}^{t / 2}\left\langle\left(\begin{array}{c}
\chi>0\left|\tilde{U}^{(t)}\right|^{4}(s) \\
-\chi_{>0}\left|\tilde{U}^{(t)}\right|^{4}(s)
\end{array}\right), \phi(x, \xi)\right\rangle
\end{aligned}
$$

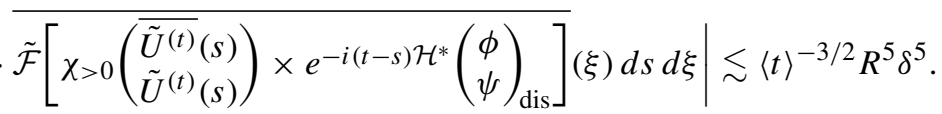

Now consider the difficult oscillatory part. Decompose

$$
\begin{aligned}
& \int_{0}^{\infty} \int_{0}^{t / 2}\left\langle\left(\begin{array}{c}
\chi>0\left|\tilde{U}^{(t)}\right|^{4}(s) \\
-\chi>0\left|\tilde{U}^{(t)}\right|^{4}(s)
\end{array}\right), s(\xi) e^{i x \xi} \underline{e}\right\rangle \\
& \overline{\tilde{\mathcal{F}}\left[\chi>0\left(\begin{array}{c}
\overline{\tilde{U}^{(t)}}(s) \\
\tilde{U}^{(t)}(s)
\end{array}\right) \times e^{-i(t-s) \mathcal{H}^{*}}\left(\begin{array}{c}
\phi \\
\psi
\end{array}\right)_{\mathrm{dis}}\right]}(\xi) d s d \xi \\
& =\int_{0}^{\infty} \int_{0}^{t / 2}\left\langle\left(\begin{array}{c}
P_{\geq a}\left[\chi_{>0}\left|\tilde{U}^{(t)}\right|^{2}(s)\right] P_{\geq a}\left[\left|\tilde{U}^{(t)}\right|^{2}\right] \\
-P_{\geq a}\left[\chi_{>0}\left|\tilde{U}^{(t)}\right|^{2}(s)\right] P_{\geq a}\left[\left|\tilde{U}^{(t)}\right|^{2}\right]
\end{array}\right), s(\xi) e^{i x \xi} \underline{e}\right\rangle \\
& \overline{\tilde{\mathcal{F}}\left[\chi>0\left(\begin{array}{c}
\overline{\tilde{U}^{(t)}}(s) \\
\tilde{U}^{(t)}(s)
\end{array}\right) \times e^{-i(t-s) \mathcal{H}^{*}}\left(\begin{array}{l}
\phi \\
\psi
\end{array}\right)_{\mathrm{dis}}\right]}(\xi) d s d \xi \\
& +\int_{0}^{\infty} \int_{0}^{t / 2}\left\langle\left(\begin{array}{c}
P_{\geq a}\left[\chi_{>0}\left|\tilde{U}^{(t)}\right|^{2}(s)\right] P_{<a}\left[\left|\tilde{U}^{(t)}\right|^{2}\right] \\
-P_{\geq a}\left[\chi_{>0}\left|\tilde{U}^{(t)}\right|^{2}(s)\right] P_{<a}\left[\left|\tilde{U}^{(t)}\right|^{2}\right]
\end{array}\right), s(\xi) e^{i x \xi} \underline{e}\right\rangle \\
& \overline{\tilde{\mathcal{F}}\left[\chi>0\left(\begin{array}{l}
\overline{\tilde{U}^{(t)}}(s) \\
\tilde{U}^{(t)}(s)
\end{array}\right) \times e^{-i(t-s) \mathcal{H}^{*}}\left(\begin{array}{l}
\phi \\
\psi
\end{array}\right)_{\mathrm{dis}}\right]}(\xi) d s d \xi \\
& +\int_{0}^{\infty} \int_{0}^{t / 2}\left\langle\left(\begin{array}{c}
P_{<a}\left[\chi_{>0}\left|\tilde{U}^{(t)}\right|^{2}(s)\right] P_{\geq a}\left[\left|\tilde{U}^{(t)}\right|^{2}\right] \\
-P_{<a}\left[\chi_{>0}\left|\tilde{U}^{(t)}\right|^{2}(s)\right] P_{\geq a}\left[\left|\tilde{U}^{(t)}\right|^{2}\right]
\end{array}\right), s(\xi) e^{i x \xi} \underline{e}\right\rangle \\
& \overline{\tilde{\mathcal{F}}\left[\chi>0\left(\begin{array}{l}
\overline{\tilde{U}^{(t)}}(s) \\
\tilde{U}^{(t)}(s)
\end{array}\right) \times e^{-i(t-s) \mathcal{H}^{*}}\left(\begin{array}{c}
\phi \\
\psi
\end{array}\right)_{\mathrm{dis}}\right]}(\xi) d s d \xi \\
& +\int_{0}^{\infty} \int_{0}^{t / 2}\left\langle\left(\begin{array}{c}
P_{<a}\left[\chi_{>0}\left|\tilde{U}^{(t)}\right|^{2}(s)\right] P_{<a}\left[\left|\tilde{U}^{(t)}\right|^{2}\right] \\
-P_{<a}\left[\chi_{>0}\left|\tilde{U}^{(t)}\right|^{2}(s)\right] P_{<a}\left[\left|\tilde{U}^{(t)}\right|^{2}\right]
\end{array}\right), s(\xi) e^{i x \xi} \underline{e}\right\rangle \\
& \overline{\tilde{\mathcal{F}}\left[\chi>0\left(\begin{array}{c}
\overline{\tilde{U}^{(t)}}(s) \\
\tilde{U}^{(t)}(s)
\end{array}\right) \times e^{-i(t-s) \mathcal{H}^{*}}\left(\begin{array}{c}
\phi \\
\psi
\end{array}\right)_{\mathrm{dis}}\right]}(\xi) d s d \xi .
\end{aligned}
$$

The cutoff $a$ here will be later chosen to be $\langle s\rangle^{-3 / 4}$. We treat each of the above terms separately. Start with the first, the high-high case: note that we can write 


$$
\begin{aligned}
\tilde{\mathcal{F}}\left[\chi>0\left(\begin{array}{c}
\overline{\tilde{U}^{(t)}}(s) \\
\tilde{U}^{(t)}(s)
\end{array}\right)\right. & \left.\times e^{-i(t-s) \mathcal{H}^{*}}\left(\begin{array}{c}
\phi \\
\psi
\end{array}\right)_{\mathrm{dis}}\right](\xi) \\
& =\left\langle\chi>0\left(\begin{array}{c}
\overline{\tilde{U}^{(t)}}(s) \\
\tilde{U}^{(t)}(s)
\end{array}\right) \times e^{-i(t-s) \mathcal{H}^{*}}\left(\begin{array}{c}
\phi \\
\psi
\end{array}\right)_{\mathrm{dis}}, s(\xi) e^{i x \xi} \underline{e}+\phi(x, \xi)\right\rangle .
\end{aligned}
$$

Then we first estimate the contribution of the elliptic component

$$
\begin{aligned}
& \mid \int_{0}^{\infty} \int_{0}^{t / 2}\left\langle\left(\begin{array}{c}
P_{\geq a}\left[\chi_{>0}\left|\tilde{U}^{(t)}\right|^{2}(s)\right] P_{\geq a}\left[\left|\tilde{U}^{(t)}\right|^{2}\right] \\
-P_{\geq a}\left[\chi_{>0}\left|\tilde{U}^{(t)}\right|^{2}(s)\right] P_{\geq a}\left[\left|\tilde{U}^{(t)}\right|^{2}\right]
\end{array}\right), s(\xi) e^{i x \xi} \underline{e}\right\rangle
\end{aligned}
$$

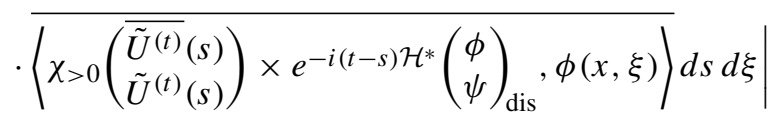

$$
\begin{aligned}
& \lesssim \int_{0}^{t / 2}\langle s\rangle^{-3+\epsilon\left(\delta_{3}\right)}\langle t-s\rangle^{-3 / 2} d s \lesssim\langle t\rangle^{-3 / 2} .
\end{aligned}
$$

Hence we reduce to estimating the expression

$$
\begin{aligned}
& \int_{0}^{\infty} \int_{0}^{t / 2}\left\langle\left(\begin{array}{c}
P_{\geq a}\left[\chi_{>0}\left|\tilde{U}^{(t)}\right|^{2}(s)\right] P_{\geq a}\left[\left|\tilde{U}^{(t)}\right|^{2}\right] \\
-P_{\geq a}\left[\chi_{>0}\left|\tilde{U}^{(t)}\right|^{2}(s)\right] P_{\geq a}\left[\left|\tilde{U}^{(t)}\right|^{2}\right]
\end{array}\right), s(\xi) e^{i x \xi} \underline{e}\right\rangle \\
& \cdot \overline{\left\langle\chi>0\left(\begin{array}{c}
\overline{\tilde{U}^{(t)}}(s) \\
\tilde{U}^{(t)}(s)
\end{array}\right) \times e^{-i(t-s) \mathcal{H}^{*}}\left(\begin{array}{c}
\phi \\
\psi
\end{array}\right)_{\mathrm{dis}}, s(\xi) e^{i x \xi} \underline{e}\right\rangle} d \xi .
\end{aligned}
$$

We intend to use the ordinary Plancherel's theorem here. We break this integral into two by including a multiplier $\phi_{\left(t^{-1000}, t^{1000}\right)}(\xi)$ or $\chi_{>0}(\xi)-\phi_{\left(t^{-1000}, t^{1000}\right)}(\xi)$, where the function $\phi_{\left(t^{-1000}, t^{1000}\right)}(\xi)$ smoothly localizes to the interval $\left(t^{-1000}, t^{1000}\right)$. It is easily seen that contribution obtained upon including the latter is very small (bounded by $\langle t\rangle^{-500}$ ), whence we may focus on the contribution of the former. By choosing $\phi_{\left(t^{-1000}, t^{1000}\right)}(\xi)$ suitably, we may assume that its Fourier transform has $L^{1}$-mass bounded by $\lesssim \log t$. Now denote the Fourier multiplier with symbol $s(\xi)^{2} \phi_{\left(t^{-1000}, t^{1000}\right)}(\xi)$ by $\Pi_{\left(t^{-1000}, t^{1000}\right)}$. Using ordinary Plancherel, we now reduce to estimating

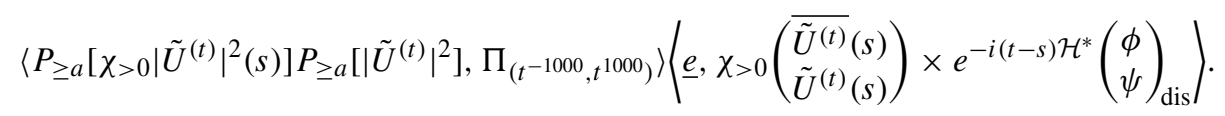

We claim that

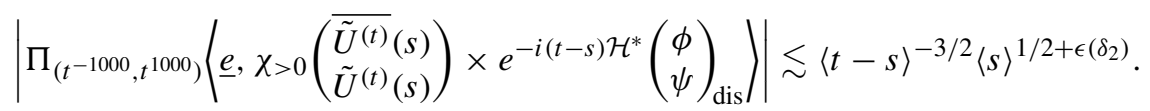

This follows from $\|x \tilde{U}(s)\|_{L_{x}^{\infty}} \lesssim\langle s\rangle^{1 / 2+\epsilon\left(\delta_{2}\right)}$, which in turn is a consequence of

$$
\begin{aligned}
\|x \tilde{U}\|_{L_{x}^{\infty}} & \leq\left\|\left(x+2 i s \partial_{x}\right) \tilde{U}\right\|_{L_{x}^{\infty}}+\left\|2 i s \partial_{x} \tilde{U}\right\|_{L_{x}^{\infty}} \\
& \lesssim\left\|\left(x+2 i s \partial_{x}\right) \partial_{x} \tilde{U}\right\|_{L_{x}^{2}}+s\left\|\partial_{x} \tilde{U}\right\|_{L_{x}^{\infty}}+\left\|\left(x+2 i s \partial_{x}\right) \tilde{U}\right\|_{L_{x}^{2}}
\end{aligned}
$$


and the following bounds, the second of which we establish later (see the next lemma):

$$
\left\|\partial_{x} \tilde{U}(s)\right\|_{L_{x}^{\infty}} \lesssim\langle s\rangle^{-1 / 2+\epsilon\left(\delta_{2}\right)}, \quad\left\|\left(x+2 i s \partial_{x}\right) \nabla \tilde{U}\right\|_{L_{x}^{2}} \lesssim\langle s\rangle^{1 / 2+\epsilon\left(\delta_{2}\right)}
$$

Now consider

$$
P_{\geq a}\left[\chi>0\left|\tilde{U}^{(t)}\right|^{2}(s)\right] P_{\geq a}\left[\left|\tilde{U}^{(t)}\right|^{2}\right]
$$

Observe that

$$
P_{\geq a}\left[\chi>0\left|\tilde{U}^{(t)}\right|^{2}(s)\right]=P_{\geq a} \partial_{x} \Delta^{-1}\left[\partial_{x}\left(\chi_{>0}\right)\left|\tilde{U}^{(t)}\right|^{2}(s)\right]+P_{\geq a} \partial_{x} \Delta^{-1}\left[\chi_{>0} \partial_{x}\left[\left|\tilde{U}^{(t)}\right|^{2}(s)\right]\right] .
$$

Note that

$$
\partial_{x}(\chi>0)\left|\tilde{U}^{(t)}\right|^{2}(s)=\delta_{0}\left|\tilde{U}^{(t)}(0)\right|^{2},
$$

whence

$$
\left\|P_{\geq a} \partial_{x} \Delta^{-1}\left[\partial_{x}(\chi>0)\left|\tilde{U}^{(t)}\right|^{2}(s)\right]\right\|_{L_{x}^{1}} \lesssim a^{-1}\langle s\rangle^{-3+\epsilon\left(\delta_{3}\right)} .
$$

Next, use the fact that

$$
\begin{aligned}
i s \partial_{x}\left[\left|\tilde{U}^{(t)}\right|^{2}\right] & =i s \partial_{x} \tilde{U}^{(t)} \overline{\tilde{U}^{(t)}}-\tilde{U}^{(t)} \overline{i s \partial_{x} \tilde{U}^{(t)}} \\
& =\left(i s \partial_{x}+\frac{x}{2}\right) \tilde{U}^{(t)} \overline{\tilde{U}^{(t)}}-\tilde{U}^{(t)} \overline{\left(i s \partial_{x}+\frac{x}{2}\right) \tilde{U}^{(t)}}
\end{aligned}
$$

whence we get

$$
\left\|P_{\geq a} \partial_{x} \Delta^{-1}\left[\chi>0 \partial_{x}\left[\left|\tilde{U}^{(t)}\right|^{2}(s)\right]\right]\right\|_{L_{x}^{2}} \lesssim a^{-1}\langle s\rangle^{-1}\langle s\rangle^{-1 / 2} .
$$

Arguing similarly for $P_{\geq a}\left[\left|\tilde{U}^{(t)}\right|^{2}\right]$, one gets

$$
\left\|P_{\geq a}\left[\chi_{>0}\left|\tilde{U}^{(t)}\right|^{2}\right] P_{\geq a}\left[\left|\tilde{U}^{(t)}\right|^{2}\right]\right\|_{L_{x}^{1}} \lesssim a^{-2}\langle s\rangle^{-3} .
$$

Combining with the bound on $\left(\begin{array}{c}\overline{\tilde{U}^{(t)}} \\ \tilde{U}^{(t)}\end{array}\right) \times e^{-i(t-s)} \mathcal{H}^{*}\left(\begin{array}{l}\phi \\ \psi\end{array}\right)_{\text {dis }}$ given above, we can bound the whole expression by

$$
\begin{aligned}
\left|\left\langle P_{\geq a}\left[\chi_{>0}\left|\tilde{U}^{(t)}\right|^{2}(s)\right] P_{\geq a}\left[\left|\tilde{U}^{(t)}\right|^{2}\right], \Pi_{\left(t^{-1000}, t^{1000)}\right\rangle}\right\rangle\left\langle\underline{e}, \chi_{>0}\left(\begin{array}{c}
\overline{\tilde{U}^{(t)}}(s) \\
\tilde{U}^{(t)}(s)
\end{array}\right) \cdot e^{-i(t-s) \mathcal{H}^{*}}\left(\begin{array}{c}
\phi \\
\psi
\end{array}\right)_{\mathrm{dis}}\right\rangle\right| \\
\lesssim a^{-2}\langle s\rangle^{-3}\langle s\rangle^{1 / 2+\epsilon\left(\delta_{2}\right)}\langle t-s\rangle^{-3 / 2}
\end{aligned}
$$

which yields something almost integrable in $s$ upon omitting the factor $\langle t-s\rangle^{-3 / 2}$ provided we choose $a=\langle s\rangle^{-3 / 4}$. This is good enough since by assumption $\delta_{3} \gg \delta_{2}$.

We now consider the other extreme, the case of low-low frequency interactions, i.e., the expression

$$
\begin{aligned}
& \int_{0}^{\infty} \int_{0}^{t / 2}\left\langle\left(\begin{array}{c}
P_{<a}\left[\chi_{>0}\left|\tilde{U}^{(t)}\right|^{2}(s)\right] P_{<a}\left[\left|\tilde{U}^{(t)}\right|^{2}\right] \\
-P_{<a}\left[\chi_{>0}\left|\tilde{U}^{(t)}\right|^{2}(s)\right] P_{<a}\left[\left|\tilde{U}^{(t)}\right|^{2}\right]
\end{array}\right), s(\xi) e^{i x \xi} \underline{e}\right\rangle
\end{aligned}
$$

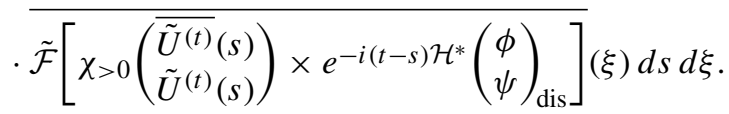


We can express $\tilde{\mathcal{F}}(\ldots)$ as before, and only the term $s(\xi) e^{i x \xi} \underline{e}$ in the Fourier basis matters. On account of the fact that

$$
\begin{aligned}
& \left\langle\left(\begin{array}{c}
P_{<a}\left[\chi>0\left|\tilde{U}^{(t)}\right|^{2}(s)\right] P_{<a}\left[\left|\tilde{U}^{(t)}\right|^{2}\right] \\
-P_{<a}\left[\chi_{>0}\left|\tilde{U}^{(t)}\right|^{2}(s)\right] P_{<a}\left[\left|\tilde{U}^{(t)}\right|^{2}\right]
\end{array}\right), s(\xi) e^{i x \xi} \underline{e}\right\rangle \\
& \left.=\chi_{<a+O(1)}(\xi) \mid\left(\begin{array}{c}
P_{<a}\left[\chi_{>0}\left|\tilde{U}^{(t)}\right|^{2}(s)\right] P_{<a}\left[\left|\tilde{U}^{(t)}\right|^{2}\right] \\
-P_{<a}\left[\chi>0\left|\tilde{U}^{(t)}\right|^{2}(s)\right] P_{<a}\left[\left|\tilde{U}^{(t)}\right|^{2}\right]
\end{array}\right), s(\xi) e^{i x \xi} \underline{e}\right\},
\end{aligned}
$$

we can estimate

$$
\begin{aligned}
& \mid \int_{0}^{\infty}\left\langle\left(\begin{array}{c}
P_{<a}\left[\chi_{>0}\left|\tilde{U}^{(t)}\right|^{2}(s)\right] P_{<a}\left[\left|\tilde{U}^{(t)}\right|^{2}\right] \\
-P_{<a}\left[\chi_{>0}\left|\tilde{U}^{(t)}\right|^{2}(s)\right] P_{<a}\left[\left|\tilde{U}^{(t)}\right|^{2}\right]
\end{array}\right), s(\xi) e^{i x \xi} \underline{e}\right\rangle \\
& \overline{\tilde{\mathcal{F}}\left[\chi>0\left(\begin{array}{c}
\overline{\tilde{U}^{(t)}}(s) \\
\tilde{U}^{(t)}(s)
\end{array}\right) \times e^{-i(t-s) \mathcal{H}^{*}}\left(\begin{array}{c}
\phi \\
\psi
\end{array}\right)_{\mathrm{dis}}\right]}(\xi) d \xi \mid \lesssim a^{2}\langle s\rangle^{-3 / 2}\langle s\rangle\langle t-s\rangle^{-3 / 2},
\end{aligned}
$$

which for $a \sim\langle s\rangle^{-3 / 4}$ can be integrated in $s$ to yield the bound $\langle t\rangle^{-3 / 2}$.

Finally, we consider the mixed case, i.e., the expression

$$
\begin{aligned}
& \int_{0}^{\infty} \int_{0}^{t / 2}\left\langle\left(\begin{array}{l}
P_{\geq a}\left[\chi_{>0}\left|\tilde{U}^{(t)}\right|^{2}(s)\right] P_{<a}\left[\left|\tilde{U}^{(t)}\right|^{2}\right] \\
-P_{\geq a}\left[\chi_{>0}\left|\tilde{U}^{(t)}\right|^{2}(s)\right] P_{<a}\left[\left|U^{2}\right|\right]
\end{array}\right), s(\xi) e^{i x \xi} \underline{e}\right\rangle \\
& \overline{\tilde{\mathcal{F}}\left[\chi>0\left(\begin{array}{l}
\overline{\tilde{U}^{(t)}}(s) \\
\tilde{U}^{(t)}(s)
\end{array}\right) \times e^{-i(t-s) \mathcal{H}^{*}}\left(\begin{array}{l}
\phi \\
\psi
\end{array}\right)_{\mathrm{dis}}\right]}(\xi) d s d \xi .
\end{aligned}
$$

We proceed as before, simplifying $\tilde{\mathcal{F}}(\ldots)$ by discarding the Schwartz term in the Fourier basis (as this case is again easy to handle as seen before), and using the ordinary Plancherel's theorem to translate this to the physical side. Arguing as before, we may do this by including a multiplier $\Pi_{\left(t^{-1000}, t^{1000}\right)}$ which is given by a kernel of $L^{1}$-mass $\lesssim$ $\log t$. We indicate this by replacing the functions $P_{\geq a}\left[\chi_{>0}\left|\tilde{U}^{(t)}\right|^{2}(s)\right]$ and $P_{<a}\left[\left|\tilde{U}^{(t)}\right|^{2}\right]$ by translates, $T_{z} P_{\geq a}\left[\chi_{>0}\left|\tilde{U}^{(t)}\right|^{2}(s)\right]$ and $T_{z} P_{<a}\left[\left|\tilde{U}^{(t)}\right|^{2}\right]$, where $\left(T_{z} f\right)(x):=f(x+z)$. The integration over $z$ in the end will cost $\lesssim \log t$. Thus we now need to consider the following expression:

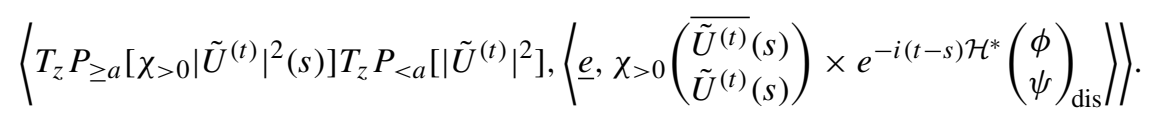

We rearrange the terms here:

$$
\left\langle T_{z} P_{<a}\left[\left|\tilde{U}^{(t)}\right|^{2}\right], T_{z} P_{\geq a}\left[\chi_{>0}\left|\tilde{U}^{(t)}\right|^{2}(s)\right]\left\langle\underline{\underline{e}, \chi>0}\left(\begin{array}{l}
\overline{\tilde{U}^{(t)}}(s) \\
\tilde{U}^{(t)}(s)
\end{array}\right) \times e^{-i(t-s) \mathcal{H}^{*}}\left(\begin{array}{l}
\phi \\
\psi
\end{array}\right)_{\mathrm{dis}}\right)\right\rangle .
$$

Revert to vectorial notation:

$$
\left\langle\left(\begin{array}{c}
T_{z} P_{<a}\left[\left|\tilde{U}^{(t)}\right|^{2}\right] \\
0
\end{array}\right),\left(\begin{array}{c}
T_{z} P_{\geq a}\left[\chi>0\left|\tilde{U}^{(t)}\right|^{2}(s)\right]\left(\underline{e}, \chi>0\left(\begin{array}{c}
\left(\overline{\tilde{U}^{(t)}}(s)\right. \\
\tilde{U}^{(t)}(s)
\end{array}\right) \times e^{-i(t-s) \mathcal{H}^{*}}\left(\begin{array}{c}
\phi \\
\psi
\end{array}\right)_{\mathrm{dis}}\right) \\
0
\end{array}\right)\right\rangle .
$$


We break this into two portions:

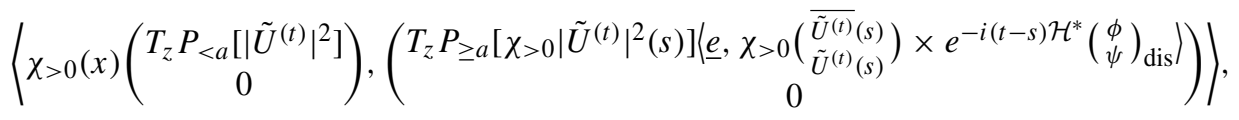

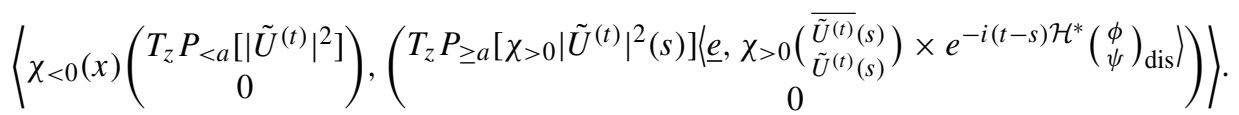

As these can be treated similarly, we consider only the first. Our first step consists in reducing the factor $\chi_{>0}(x)\left(T_{z} P_{<a}\left[\left|\tilde{U}^{(t)}\right|^{2}\right]\right)$ to its dispersive part. Note that if we substitute $\left\langle\chi>0(x)\left(\begin{array}{c}T_{z} P_{<a}\left[\left|\tilde{U}^{(t)}\right|^{2}\right] \\ 0\end{array}\right), \xi_{k(j)}\right\rangle \eta_{j}$ for this expression instead, we can estimate

$$
\begin{aligned}
& \left\langle\left\langle\chi>0(x)\left(\begin{array}{c}
T_{z} P_{<a}\left[\left|\tilde{U}^{(t)}\right|^{2}\right] \\
0
\end{array}\right), \xi_{k(j)}\right\rangle \eta_{j}\right.
\end{aligned}
$$

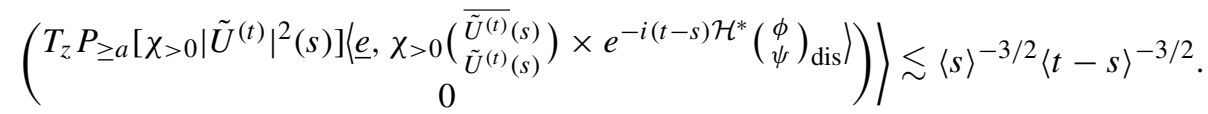

This can be integrated in $s$ to yield the upper bound $\lesssim\langle t\rangle^{-3 / 2}$. Now, with the left factor reduced to its dispersive part, invoking the distorted Plancherel's Theorem 2.3. we need to estimate

$$
\begin{aligned}
& \sum_{ \pm} \int_{-\infty}^{\infty} \mathcal{F}_{ \pm}\left[\chi_{>0}(x)\left(\begin{array}{c}
T_{z} P_{<a}\left[\left|\tilde{U}^{(t)}\right|^{2}\right] \\
0
\end{array}\right)\right](\xi)
\end{aligned}
$$

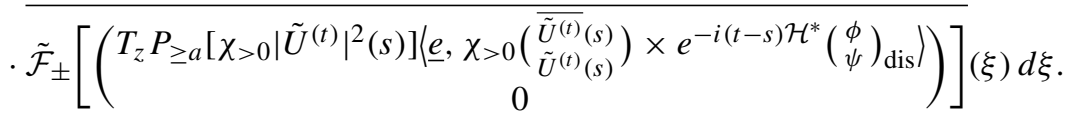

We may and shall treat the case + , and omit the subscript for simplicity. As before, we need to subdivide the $\xi$-integration into two contributions, one from $(-\infty, 0]$, the other from $[0, \infty)$. We treat here the contribution from the latter, that from the former being more complicated and treated below. We decompose

$$
\begin{aligned}
\mathcal{F}[\chi>0(x) & \left.\left(\begin{array}{c}
T_{z} P_{<a}\left[\left|\tilde{U}^{(t)}\right|^{2}\right] \\
0
\end{array}\right)\right](\xi) \\
& =\mathcal{F}\left[\chi_{>0}(x)\left(\begin{array}{c}
T_{z}\left[\left|\tilde{U}^{(t)}\right|^{2}\right] \\
0
\end{array}\right)\right](\xi)-\mathcal{F}\left[\chi_{>0}(x)\left(\begin{array}{c}
T_{z} P_{\geq a}\left[\left|\tilde{U}^{(t)}\right|^{2}\right] \\
0
\end{array}\right)\right](\xi) .
\end{aligned}
$$

Substituting the second summand results in an expression which can be treated as in the high-high case. Thus substitute the first summand on the right, $\mathcal{F}\left[\chi>0(x)\left(\begin{array}{c}T_{z}\left[\left|\tilde{U}^{(t)}\right|^{2}\right] \\ 0\end{array}\right)\right](\xi)$. One explicitly writes out the Fourier transform, and may discard the contribution from the local part $\phi(x, \xi)$ of the Fourier basis, reasoning as before. Then one obtains the following expression: 


$$
\begin{aligned}
& \int_{0}^{\infty}\left\langle\chi_{>0}(x)\left(\begin{array}{c}
T_{z}\left[\left|\tilde{U}^{(t)}\right|^{2}\right] \\
0
\end{array}\right), s(\xi) e^{i x \xi} \underline{e}\right\rangle
\end{aligned}
$$

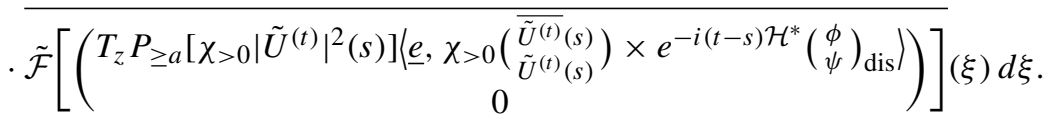

In this, break $\chi_{>0}(x)\left(\begin{array}{c}T_{z}\left[\left|\tilde{U}^{(t)}\right|^{2}\right] \\ 0\end{array}\right)$ into two parts, a large frequency and a small frequency part:

$$
\chi_{>0}(x)\left(\begin{array}{c}
T_{z}\left[\left|\tilde{U}^{(t)}\right|^{2}\right] \\
0
\end{array}\right)=P_{\geq a}\left[\chi_{>0}(x)\left(\begin{array}{c}
T_{z}\left[\left|\tilde{U}^{(t)}\right|^{2}\right] \\
0
\end{array}\right)\right]+P_{<a}\left[\chi_{>0}(x)\left(\begin{array}{c}
T_{z}\left[\left|\tilde{U}^{(t)}\right|^{2}\right] \\
0
\end{array}\right)\right] .
$$

Consider the first summand on the right: we replace it by

$$
\partial_{x} \Delta^{-1} P_{\geq a}\left[\delta_{0}(x)\left(\begin{array}{c}
T_{z}\left[\left|\tilde{U}^{(t)}\right|^{2}\right] \\
0
\end{array}\right)\right]+\partial_{x} \Delta^{-1} P_{\geq a}\left[\chi>0(x)\left(\begin{array}{c}
T_{z} \partial_{x}\left[\left|\tilde{U}^{(t)}\right|^{2}\right] \\
0
\end{array}\right)\right] .
$$

Observe that the $a^{-1}$ from the operator $\partial_{x} \Delta^{-1} P_{\geq a}$ is counteracted by the factor $s(\xi)$ above. In order to treat the contribution from the first summand, subdivide the interval $[a, \infty)$ into dyadic intervals, and sum. Thus we need to estimate

$$
\begin{aligned}
& \sum_{2^{j} \geq a} \int_{0}^{\infty}\left\langle\left(\begin{array}{c}
\partial_{x} \Delta^{-1} P_{2^{j}}\left[\delta_{0}(x) T_{z}\left[\left|\tilde{U}^{(t)}\right|^{2}\right]\right] \\
0
\end{array}\right), s(\xi) e^{i x \xi} \underline{e}\right\rangle \\
& \overline{\left.. \tilde{\mathcal{F}}\left[\left(\begin{array}{c}
T_{z} P_{\geq a}\left[\chi>0\left|\tilde{U}^{(t)}\right|^{2}(s)\right] \mid \underline{e}, \chi>0 \\
0
\end{array}\left(\begin{array}{c}
\tilde{\tilde{U}}^{(t)}(s) \\
\tilde{U}^{(s)}
\end{array}\right) \times e^{-i(t-s) \mathcal{H}^{*}}\left(\begin{array}{l}
\phi \\
\psi
\end{array}\right)_{\mathrm{dis}}\right)\right)\right]}(\xi) d \xi .
\end{aligned}
$$

We have

$$
\|\left(\left(\begin{array}{c}
\partial_{x} \Delta^{-1} P_{2 j}\left[\delta_{0}(x) T_{z}\left[\left|\tilde{U}^{(t)}\right|^{2}\right]\right] \\
0
\end{array}\right), s(\xi) e^{i x \xi} \underline{e}\right\rangle \mid \lesssim \min \left\{2^{-j}, 1\right\} s^{-1} .
$$

Moreover,

$$
\begin{aligned}
& \left\|\tilde{\mathcal{F}}\left[\left(\begin{array}{c}
T_{z} P_{\geq a}\left[\chi_{>0}\left|\tilde{U}^{(t)}\right|^{2}(s)\right]\left(\underline{e}, \chi_{>0}\left(\begin{array}{c}
\overline{\tilde{U}^{(t)}}(s) \\
0 \\
0
\end{array}\right) \times e^{-i(t) s)}(s)\right. \\
\left.\mathcal{H}^{*}\left(\begin{array}{l}
\phi \\
\psi
\end{array}\right)_{\mathrm{dis}}\right)
\end{array}\right)\right](\xi)\right\|_{L_{\xi}^{2}} \\
& \lesssim a^{-1}\langle s\rangle^{-3 / 2}\langle s\rangle^{1 / 2+\epsilon\left(\delta_{2}\right)}\langle t-s\rangle^{-3 / 2} \text {. }
\end{aligned}
$$

Thus, by Hölder's inequality,

$$
\begin{aligned}
& \mid \int_{0}^{\infty}\left\langle\left(\begin{array}{c}
\partial_{x} \Delta^{-1} P_{2^{j}}\left[\delta_{0}(x) T_{z}\left[\left|\tilde{U}^{(t)}\right|^{2}\right]\right] \\
0
\end{array}\right), s(\xi) e^{i x \xi} \underline{e}\right\rangle
\end{aligned}
$$

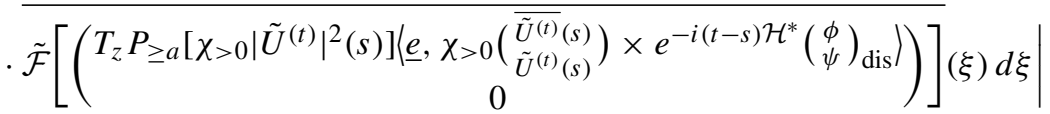

$$
\begin{aligned}
& \lesssim \min \left\{2^{-j / 2}, 1\right\}\langle s\rangle^{-1} a^{-1}\langle s\rangle^{-3 / 2}\langle s\rangle^{1 / 2+\epsilon\left(\delta_{2}\right)}\langle t-s\rangle^{-3 / 2} \text {. }
\end{aligned}
$$


Summing over $j$ costs at most $\log s$, whence substituting $a=\langle s\rangle^{-3 / 4}$ and integrating in $s$ yields the upper bound $\lesssim\langle t\rangle^{-3 / 2+\delta_{3}}$, as desired. If, on the other hand, we substitute $\partial_{x} \Delta^{-1} P_{\geq a}\left[\chi_{>0}(x) T_{z} \partial_{x}\left[\left|\tilde{U}^{(t)}\right|^{2}\right]\right.$, we argue just as for the high-high case. Now consider the expression

$$
\begin{aligned}
& \int_{0}^{\infty}\left\langle P_{<a}\left[\chi>0(x)\left(\begin{array}{c}
T_{z}\left[\left|\tilde{U}^{(t)}\right|^{2}\right] \\
0
\end{array}\right)\right], s(\xi) e^{i x \xi} \underline{e}\right\rangle
\end{aligned}
$$

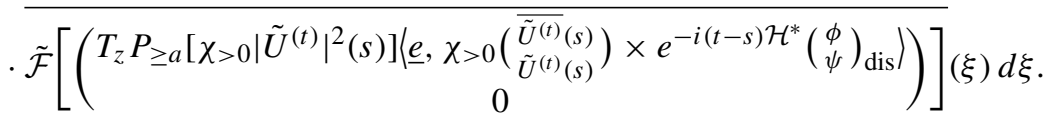

Estimate

$$
\begin{aligned}
& \left\|\left\langle P_{<a}\left[\chi_{>0}(x)\left(\begin{array}{c}
T_{z}\left[\left|\tilde{U}^{(t)}\right|^{2}\right] \\
0
\end{array}\right)\right], s(\xi) e^{i x \xi} \underline{e}\right)\right\|_{L_{\xi}^{2}} \lesssim a\langle s\rangle^{-1 / 2}, \\
& \| \mathcal{F}\left[\left(\begin{array}{c}
\left.\left.T_{z} P_{\geq a}\left[\chi>0\left|\tilde{U}^{(t)}\right|^{2}(s)\right]\left\langle\underline{e}, \chi_{>0}\left(\begin{array}{c}
\overline{\tilde{U}^{(t)}}(s) \\
\tilde{U}^{(t)}(s)
\end{array}\right) \times e^{-i(t-s) \mathcal{H}^{*}}\left(\begin{array}{c}
\phi \\
\psi
\end{array}\right)_{\mathrm{dis}}\right\rangle\right)\right](\xi) \\
0
\end{array} \|_{L_{\xi}^{2}}\right.\right. \\
& \lesssim a^{-1}\langle s\rangle^{-3 / 2}\langle s\rangle^{1 / 2+\epsilon\left(\delta_{2}\right)}\langle t-s\rangle^{-3 / 2} \text {. }
\end{aligned}
$$

Putting these together results in the upper bound

$$
\begin{aligned}
& \mid \int_{0}^{\infty}\left\langle P_{<a}\left[\chi_{>0}(x)\left(\begin{array}{c}
T_{z}\left[\left|\tilde{U}^{(t)}\right|^{2}\right] \\
0
\end{array}\right)\right], s(\xi) e^{i x \xi} \underline{e}\right\rangle
\end{aligned}
$$

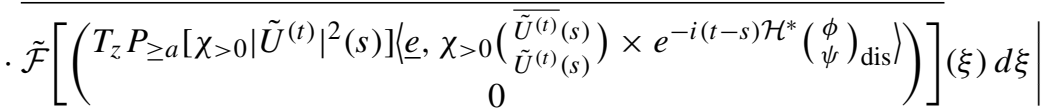

$$
\begin{aligned}
& \lesssim a\langle s\rangle^{-1 / 2} a^{-1}\langle s\rangle^{-3 / 2}\langle s\rangle^{1 / 2+\epsilon\left(\delta_{2}\right)}\langle t-s\rangle^{-3 / 2} \lesssim\langle s\rangle^{-3 / 2+\epsilon\left(\delta_{2}\right)}\langle t-s\rangle^{-3 / 2},
\end{aligned}
$$

which upon integration in $s$ again yields the desired upper bound $\langle t\rangle^{-3 / 2+\delta_{3}}$. The case when the $\xi$-variable is restricted to $(-\infty, 0]$ in the mixed case will be treated further below.

Now we consider the case $\xi \in(-\infty, 0]$, and hence the expression

$$
\int_{-\infty}^{0} \int_{0}^{t / 2} \mathcal{F}\left(\begin{array}{c}
\chi_{>0}\left|\tilde{U}^{(t)}\right|^{4}(s) \\
-\chi_{>0}\left|\tilde{U}^{(t)}\right|^{4}(s)
\end{array}\right)(\xi) \overline{\mathcal{F}} \overline{\left[\chi_{>0}\left(\begin{array}{c}
\overline{\tilde{U}^{(t)}}(s) \\
\tilde{U}^{(t)}(s)
\end{array}\right) \times e^{-i(t-s) \mathcal{H}^{*}}\left(\begin{array}{c}
\phi \\
\psi
\end{array}\right)_{\mathrm{dis}}\right]}(\xi) d s d \xi
$$

Reformulate this as

$$
\begin{aligned}
& \int_{-\infty}^{0} \int_{0}^{t / 2}\left\langle\left(\begin{array}{c}
\chi>0\left|\tilde{U}^{(t)}\right|^{4}(s) \\
-\chi>0\left|\tilde{U}^{(t)}\right|^{4}(s)
\end{array}\right),\left(e^{i x \xi}-e^{-i x \xi}\right) \underline{e}+(1+r(-\xi)) e^{-i x \xi} \underline{e}+\phi(x, \xi)\right\rangle
\end{aligned}
$$

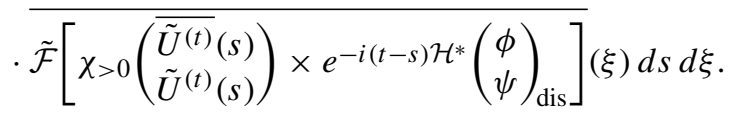


We can treat the contribution of $\phi(x, \xi)$ just as we did before. Futhermore, note that $|1+r(-\xi)|=O(|\xi|$ ) around $\xi=0$ (see Theorem 2.2), whence we can treat the contribution of this part just like we did for the transmission part before. The remaining part is more difficult and we break it into a number of contributions:

$$
\begin{aligned}
& \int_{-\infty}^{0} \int_{0}^{t / 2}\left\langle\left(\begin{array}{c}
P_{\geq a}\left[\chi_{>0}\left|\tilde{U}^{(t)}\right|^{2}(s)\right] P_{\geq a}\left[\left|\tilde{U}^{(t)}\right|^{2}(s)\right] \\
-P_{\geq a}\left[\chi_{>0}\left|\tilde{U}^{(t)}\right|^{2}(s)\right] P_{\geq a}\left[\left|\tilde{U}^{(t)}\right|^{2}(s)\right]
\end{array}\right),\left(e^{i x \xi}-e^{-i x \xi}\right) \underline{e}\right\rangle \\
& \overline{\tilde{\mathcal{F}}\left[\chi>0\left(\begin{array}{c}
\overline{\tilde{U}^{(t)}}(s) \\
\tilde{U}^{(t)}(s)
\end{array}\right) \times e^{-i(t-s) \mathcal{H}^{*}}\left(\begin{array}{c}
\phi \\
\psi
\end{array}\right)\right]}(\xi) d s d \xi, \\
& \int_{-\infty}^{0} \int_{0}^{t / 2}\left\langle\left(\begin{array}{c}
P_{<a}\left[\chi_{>0}\left|\tilde{U}^{(t)}\right|^{2}(s)\right] P_{\geq a}\left[\left|\tilde{U}^{(t)}\right|^{2}(s)\right] \\
-P_{<a}\left[\chi_{>0}\left|\tilde{U}^{(t)}\right|^{2}(s)\right] P_{\geq a}\left[\left|\tilde{U}^{(t)}\right|^{2}(s)\right]
\end{array}\right),\left(e^{i x \xi}-e^{-i x \xi}\right) \underline{e}\right\rangle \\
& \overline{\tilde{\mathcal{F}}\left[\chi>0\left(\begin{array}{l}
\overline{\tilde{U}^{(t)}}(s) \\
\tilde{U}^{(t)}(s)
\end{array}\right) \times e^{-i(t-s) \mathcal{H}^{*}}\left(\begin{array}{c}
\phi \\
\psi
\end{array}\right)\right]}(\xi) d s d \xi, \\
& \int_{-\infty}^{0} \int_{0}^{t / 2}\left\langle\left(\begin{array}{c}
P_{\geq a}\left[\left.\chi_{>0} \tilde{U}^{(t)}\right|^{2}(s)\right] P_{<a}\left[\left|\tilde{U}^{(t)}\right|^{2}(s)\right] \\
-P_{\geq a}\left[\chi_{>0}\left|\tilde{U}^{(t)}\right|^{2}(s)\right] P_{<a}\left[\left|\tilde{U}^{(t)}\right|^{2}(s)\right]
\end{array}\right),\left(e^{i x \xi}-e^{-i x \xi}\right) \underline{e}\right\rangle \\
& \overline{\tilde{\mathcal{F}}\left[\chi>0\left(\begin{array}{c}
\overline{\tilde{U}^{(t)}}(s) \\
\tilde{U}^{(t)}(s)
\end{array}\right) \times e^{-i(t-s) \mathcal{H}^{*}}\left(\begin{array}{c}
\phi \\
\psi
\end{array}\right)\right]}(\xi) d s d \xi, \\
& \int_{-\infty}^{0} \int_{0}^{t / 2}\left\langle\left(\begin{array}{c}
P_{<a}\left[\chi_{>0}|U|^{2}(s)\right] P_{<a}\left[\left|\tilde{U}^{(t)}\right|^{2}(s)\right] \\
-P_{<a}\left[\chi_{>0}|U|^{2}(s)\right] P_{<a}\left[\left|\tilde{U}^{(t)}\right|^{2}(s)\right]
\end{array}\right),\left(e^{i x \xi}-e^{-i x \xi}\right) \underline{e}\right\rangle \\
& \overline{\tilde{\mathcal{F}}\left[\chi_{>0}\left(\begin{array}{c}
\overline{\tilde{U}^{(t)}}(s) \\
\tilde{U}^{(t)}(s)
\end{array}\right) \times e^{-i(t-s) \mathcal{H}^{*}}\left(\begin{array}{c}
\phi \\
\psi
\end{array}\right)\right]}(\xi) d s d \xi .
\end{aligned}
$$

Start with the first term in this list: write

$$
\begin{aligned}
\tilde{\mathcal{F}}\left[\chi>0\left(\begin{array}{c}
\overline{\tilde{U}^{(t)}}(s) \\
\tilde{U}^{(t)}(s)
\end{array}\right) \times e^{-i(t-s) \mathcal{H}^{*}}\left(\begin{array}{l}
\phi \\
\psi
\end{array}\right)\right] \\
=\left\langle\chi>0\left(\begin{array}{c}
\overline{\tilde{U}^{(t)}}(s) \\
\tilde{U}^{(t)}(s)
\end{array}\right) \times e^{-i(t-s) \mathcal{H}^{*}}\left(\begin{array}{c}
\phi \\
\psi
\end{array}\right),\left[e^{i x \xi}+r(-\xi) e^{-i x \xi}\right] \underline{e}+\phi(x, \xi)\right\rangle .
\end{aligned}
$$

The contribution of $\phi(x, \xi)$ here is again straightforward, and left out. Now one proceeds as for the transmission part $(\xi \geq 0)$ treated before, using the ordinary Plancherel's Theorem and introducing a multiplier $\Pi_{\left(t^{-1000}, t^{1000}\right)}$.

Next, we consider the low-low frequency interaction, i.e., the expression

$$
\begin{array}{r}
\int_{-\infty}^{0} \int_{0}^{t / 2}\left\langle\left(\begin{array}{c}
P_{<a}\left[\chi_{>0}\left|\tilde{U}^{(t)}\right|^{2}(s)\right] P_{<a}\left[\left|\tilde{U}^{(t)}\right|^{2}(s)\right] \\
-P_{<a}\left[\chi_{>0}\left|\tilde{U}^{(t)}\right|^{2}(s)\right] P_{<a}\left[\left|\tilde{U}^{(t)}\right|^{2}(s)\right]
\end{array}\right),\left(e^{i x \xi}-e^{-i x \xi}\right) \underline{e}\right\rangle \\
\frac{\left.\tilde{\mathcal{F}}\left[\begin{array}{c}
\chi>0 \\
\left(\overline{\tilde{U}^{(t)}}(s)\right. \\
\tilde{U}^{(t)}(s)
\end{array}\right) \times e^{-i(t-s) \mathcal{H}^{*}}\left(\begin{array}{c}
\phi \\
\psi
\end{array}\right)\right]}{(\xi) d s d \xi .}
\end{array}
$$


This calls for a different strategy than for the transmission part, since the Fourier basis in this regime does not vanish uniformly at $\xi=0$. First, we observe that

$$
\begin{aligned}
& \left\langle\left(\begin{array}{c}
P_{<a}\left[\chi>0\left|\tilde{U}^{(t)}\right|^{2}(s)\right] P_{<a}\left[\left|\tilde{U}^{(t)}\right|^{2}(s)\right] \\
-P_{<a}\left[\chi_{>0}\left|\tilde{U}^{(t)}\right|^{2}(s)\right] P_{<a}\left[\left|\tilde{U}^{(t)}\right|^{2}(s)\right]
\end{array}\right),\left(e^{i x \xi}-e^{-i x \xi}\right) \underline{e}\right\rangle \\
& =\chi_{<a+O(1)}(\xi)\left\langle\left(\begin{array}{c}
P_{<a}\left[\chi>0\left|\tilde{U}^{(t)}\right|^{2}(s)\right] P_{<a}\left[\left|\tilde{U}^{(t)}\right|^{2}(s)\right] \\
-P_{<a}\left[\chi_{>0}\left|\tilde{U}^{(t)}\right|^{2}(s)\right] P_{<a}\left[\left|\tilde{U}^{(t)}\right|^{2}(s)\right]
\end{array}\right),\left(e^{i x \xi}-e^{-i x \xi}\right) \underline{e}\right\rangle .
\end{aligned}
$$

Hence we have

$$
\left\|\left\langle\left(\begin{array}{c}
P_{<a}\left[\chi_{>0}\left|\tilde{U}^{(t)}\right|^{2}(s)\right] P_{<a}\left[\left|\tilde{U}^{(t)}\right|^{2}(s)\right] \\
-P_{<a}\left[\chi_{>0}\left|\tilde{U}^{(t)}\right|^{2}(s)\right] P_{<a}\left[\left|\tilde{U}^{(t)}\right|^{2}(s)\right]
\end{array}\right),\left(e^{i x \xi}-e^{-i x \xi}\right) \underline{e}\right)\right\|_{L_{\xi}^{1}} \lesssim a\langle s\rangle^{-1} .
$$

Notice that putting $a=\langle s\rangle^{-3 / 4}$ is not quite good enough yet to counterbalance the loss of $s$ arising when one extracts the $(t-s)^{-3 / 2}$-gain. This extra gain of $s^{-1 / 4}$ has to come from the second factor $\tilde{\mathcal{F}}(\ldots)$. For $\xi<0$ write

$$
\begin{aligned}
& \tilde{\mathcal{F}}\left[\chi>0\left(\begin{array}{c}
\overline{\tilde{U}^{(t)}} \\
\tilde{U}^{(t)}
\end{array}\right) \times e^{-i(t-s) \mathcal{H}^{*}}\left(\begin{array}{l}
\phi \\
\psi
\end{array}\right)_{\mathrm{dis}}\right](\xi) \\
& =\left\langle\chi>0\left(\begin{array}{l}
\left(\tilde{U}^{(t)}\right. \\
\tilde{U}^{(t)}
\end{array}\right) \times e^{-i(t-s)} \mathcal{H}^{*}\left(\begin{array}{c}
\phi \\
\psi
\end{array}\right)_{\mathrm{dis}},\left[e^{i x \xi}-e^{-i x \xi}\right] \underline{e}+(1+r(-\xi)) e^{-i x \xi} \underline{e}+\phi(x, \xi)\right\rangle .
\end{aligned}
$$

We first deal with the contribution of $(1+r(-\xi)) e^{-i x \xi} \underline{e}+\phi(x, \xi)$. Note that for $\xi \lesssim a$, we have

$$
\left\|\left\langle\chi>0\left(\begin{array}{c}
\overline{\tilde{U}^{(t)}} \\
\tilde{U}^{(t)}
\end{array}\right) \times e^{-i(t-s) \mathcal{H}^{*}}\left(\begin{array}{c}
\phi \\
\psi
\end{array}\right)_{\mathrm{dis}},(1+r(-\xi)) e^{-i x \xi} \underline{e}\right)\right\|_{L_{\xi}^{2}} \lesssim a\langle s\rangle^{1+\epsilon\left(\delta_{2}\right)}\langle t-s\rangle^{-3 / 2} .
$$

Combining this with

$$
\left\|\left\langle\left(\begin{array}{c}
P_{<a}\left[\chi_{>0}\left|\tilde{U}^{(t)}\right|^{2}(s)\right] P_{<a}\left[\left|\tilde{U}^{(t)}\right|^{2}(s)\right] \\
-P_{<a}\left[\chi_{>0}\left|\tilde{U}^{(t)}\right|^{2}(s)\right] P_{<a}\left[\left|\tilde{U}^{(t)}\right|^{2}(s)\right]
\end{array}\right),\left(e^{i x \xi}-e^{-i x \xi}\right) \underline{e}\right\rangle\right\|_{L_{\xi}^{2}} \lesssim\langle s\rangle^{-3 / 2}
$$

we obtain the inequality

$$
\begin{aligned}
& \mid \int_{-\infty}^{0} \int_{0}^{t / 2}\left\langle\left(\begin{array}{c}
P_{<a}\left[\chi>0\left|\tilde{U}^{(t)}\right|^{2}(s)\right] P_{<a}\left[\left|\tilde{U}^{(t)}\right|^{2}(s)\right] \\
-P_{<a}\left[\chi>0\left|\tilde{U}^{(t)}\right|^{2}(s)\right] P_{<a}\left[\left|\tilde{U}^{(t)}\right|^{2}(s)\right]
\end{array}\right),\left(e^{i x \xi}-e^{-i x \xi}\right) \underline{e}\right\rangle
\end{aligned}
$$

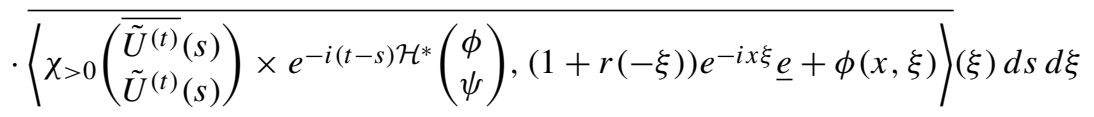

$$
\begin{aligned}
& \lesssim \int_{0}^{t / 2}\langle s\rangle^{-5 /(4+)}\langle t-s\rangle^{-3 / 2} d s \lesssim\langle t\rangle^{-3 / 2}
\end{aligned}
$$

We now reduce to estimating the contribution where $\tilde{\mathcal{F}}[\ldots]$ is replaced by the expression 


$$
\begin{aligned}
& \int_{-\infty}^{\infty}\left[e^{i x \xi}-e^{-i x \xi}\right]\left\langle\underline{\underline{e}}, \chi>0\left(\begin{array}{c}
\overline{\tilde{U}^{(t)}} \\
\tilde{U}^{(t)}
\end{array}\right) \times e^{-i(t-s) \mathcal{H}^{*}}\left(\begin{array}{l}
\phi \\
\psi
\end{array}\right)_{\mathrm{dis}}\right) d x \\
& =i \xi \int_{0}^{\infty}\left[e^{i x \xi}+e^{-i x \xi}\right]\left\langle\underline{\underline{e}}, \int_{x}^{\infty} \chi>0\left(\begin{array}{l}
\overline{\tilde{U}}^{(t)}(s) \\
\tilde{U}^{(t)}(s)
\end{array}\right) \times e^{-i(t-s) \mathcal{H}^{*}}\left(\begin{array}{c}
\phi \\
\psi
\end{array}\right)_{\mathrm{dis}}(y) d y\right) d x .
\end{aligned}
$$

It is at this point that we use the fact that $\tilde{U}$ has the structure of convex linear combination, (5.3), detailed at the beginning of the proof of the current lemma. In particular, we shall express $\tilde{U}_{\text {dis }}$ as a sum of Fresnel type integrals. First, using the distorted Fourier transform, we write

$$
\chi_{>0} e^{-i(t-s) \mathcal{H}^{*}}\left(\begin{array}{c}
\phi \\
\psi
\end{array}\right)_{\mathrm{dis}}=\sum_{ \pm} \int_{-\infty}^{\infty} e^{ \pm i(t-s)\left(\xi^{2}+1\right)} \chi_{>0} \sigma_{3} e_{ \pm}(x, \xi) \tilde{\mathcal{F}}_{ \pm}\left(\begin{array}{l}
\phi \\
\psi
\end{array}\right)(\xi) d \xi
$$

Fix the +-sign here, the --sign being treated accordingly; it is important here that the oscillatory part of $e_{-}(x, \xi)$ only has a lower component, i.e., $e_{-}(x, \xi)=e^{i x \xi} \sigma_{1} \underline{e}+\cdots$, where $\sigma_{1}=\left(\begin{array}{ll}0 & 1 \\ 1 & 0\end{array}\right), \underline{e}=\left(\begin{array}{l}1 \\ 0\end{array}\right)$, while the oscillatory part of $e_{+}(x, \xi)$ only has an upper component. Then we break the integral into two contributions:

$$
\begin{aligned}
& \int_{0}^{\infty} e^{i(t-s)\left(\xi^{2}+1\right)} \chi_{>0} \sigma_{3} e(x, \xi) \tilde{\mathcal{F}}\left(\begin{array}{l}
\phi \\
\psi
\end{array}\right)(\xi) d \xi, \\
& \int_{-\infty}^{0} e^{i(t-s)\left(\xi^{2}+1\right)} \chi>0 \sigma_{3} e(x, \xi) \tilde{\mathcal{F}}\left(\begin{array}{c}
\phi \\
\psi
\end{array}\right)(\xi) d \xi .
\end{aligned}
$$

Write the first integral 5.6 as

$$
\int_{0}^{\infty} e^{i(t-s)\left(\xi^{2}+1\right)} \chi_{>0} \sigma_{3}\left[s(\xi) e^{i x \xi} \underline{e}+\phi(x, \xi)\right] \tilde{\mathcal{F}}\left(\begin{array}{c}
\phi \\
\psi
\end{array}\right)(\xi) d \xi .
$$

Carry out an integration by parts in $\xi$, thereby replacing this by

$$
\frac{1}{t-s} \int_{0}^{\infty} e^{i(t-s)\left(\xi^{2}+1\right)} \chi_{>0}(x) \sigma_{3} \partial_{\xi}\left(\left[s(\xi) e^{i x \xi} \underline{e}+\phi(x, \xi)\right] \frac{\tilde{\mathcal{F}}\left(\begin{array}{c}
\phi \\
\psi
\end{array}\right)(\xi)}{\xi}\right) d \xi .
$$

The contribution of $\phi(x, \xi)$ here is again negligible, as is easily seen. The worst case occurs when the derivative $\partial_{\xi}$ falls on the phase $e^{i x \xi}$, costing a factor $i x$. Explicitly, this is the following expression:

$$
\frac{i x}{t-s} \int_{0}^{\infty} e^{i(t-s)\left(\xi^{2}+1\right)} \chi>0(x) \sigma_{3} s(\xi) e^{i x \xi} \underline{e} \frac{\tilde{\mathcal{F}}\left(\begin{array}{l}
\phi \\
\psi
\end{array}\right)(\xi)}{\xi} d \xi
$$

Break the $\xi$-integral into two, one over the interval $\left[0, t^{1000}\right]$, the other over its complement on $[0, \infty)$. On the latter, an additional integration by parts in $\xi$ easily furnishes more than the needed gain in $t$. On the former interval, observe that we may interpret the integral

$$
\int_{0}^{\infty} e^{i(t-s) \xi^{2}} \sigma_{3} s(\xi) e^{i x \xi} \underline{e} \frac{\tilde{\mathcal{F}}\left(\begin{array}{l}
\phi \\
\psi
\end{array}\right)(\xi)}{\xi} \chi_{<t} 1000(\xi) d \xi
$$


as a solution for the free Schrödinger equation, evaluated at time $t-s$, with initial data

$$
g(x)=\int_{0}^{\infty} \sigma_{3} s(\xi) e^{i x \xi} \underline{e} \frac{\tilde{\mathcal{F}}\left(\begin{array}{c}
\phi \\
\psi
\end{array}\right)(\xi)}{\xi} \chi_{<t} 1000(\xi) d \xi .
$$

The definition of $\tilde{\mathcal{F}}$ as well as further integrations by parts in $\xi$ reveal that this decays like $x^{-2}$ for large values of $x$, resulting in

$$
\left\|\int_{0}^{\infty} \sigma_{3} s(\xi) e^{i x \xi} \underline{e} \frac{\tilde{\mathcal{F}}\left(\begin{array}{l}
\phi \\
\psi
\end{array}\right)(\xi)}{\xi} \chi_{<t} 1000(\xi) d \xi\right\|_{L_{x}^{1}} \lesssim \log t .
$$

Thus we can now write

$$
\begin{aligned}
\frac{i x}{t-s} \int_{0}^{\infty} e^{i(t-s)\left(\xi^{2}+1\right)} \chi_{<t} 1000(\xi) \sigma_{3} s(\xi) e^{i x \xi} & \underline{e} \frac{\tilde{\mathcal{F}}\left(\begin{array}{l}
\phi \\
\psi
\end{array}\right)(\xi)}{\xi} d \xi \\
& =\frac{i e^{i(t-s)} x}{t-s} \frac{1}{\sqrt{t-s}} \int_{-\infty}^{\infty} e^{-\frac{(x-y)^{2}}{i(t-s)}} g(y) d y
\end{aligned}
$$

Next, returning to 5.7), we consider the integral

$$
\int_{-\infty}^{0} e^{i(t-s)\left(\xi^{2}+1\right)} \chi_{>0} \sigma_{3} e(x, \xi) \tilde{\mathcal{F}}\left(\begin{array}{l}
\phi \\
\psi
\end{array}\right)(\xi) d \xi .
$$

In the regime considered we can write $e(x, \xi)=\left[e^{i x \xi}-e^{-i x \xi}+(1+r(-\xi)) e^{-i x \xi}\right] \underline{e}+$ $\phi(x, \xi)$. We proceed as before, arriving (up to error terms handled as before) at the expression

$$
\begin{aligned}
\frac{i x}{t-s} \int_{-\infty}^{0} e^{i(t-s)\left(\xi^{2}+1\right)} \chi>0(x)\left[e^{i x \xi}\right. & \left.+e^{-i x \xi}\right] \underline{e} \frac{\tilde{\mathcal{F}}\left(\begin{array}{l}
\phi \\
\psi
\end{array}\right)(\xi)}{\xi} d \xi \\
& =\frac{i x}{t-s} \frac{1}{\sqrt{t-s}} \int_{-\infty}^{\infty} e^{-\frac{(x-y)^{2}}{i(t-s)}} \tilde{g}(y) d y
\end{aligned}
$$

where

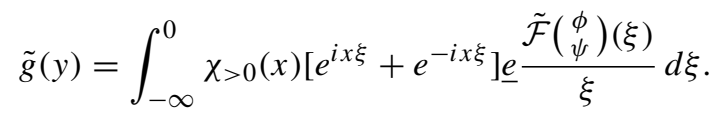

Now substitute either 5.8) or 5.9 for the right hand factor in $\left(\begin{array}{l}\overline{\tilde{U}^{(t)}} \\ \tilde{U}^{(t)}\end{array}\right) \times e^{-i(t-s) \mathcal{H}^{*}}\left(\begin{array}{l}\phi \\ \psi\end{array}\right)_{\mathrm{dis}}$. We replace the resulting $\overline{\tilde{U}^{(t)}}$ by $s \partial_{x} \overline{\tilde{U}^{(t)}}$, upon using the control over $\left\|C \tilde{U}^{(t)}\right\|_{L_{x}^{2}}$. Thus, if we substitute for example 5.9 , we need to estimate the term arising upon substituting

$$
\chi>0(x) s \partial_{x} \overline{\tilde{U}^{(t)}} \frac{e^{i(t-s)}}{t-s} \int_{-\infty}^{0} e^{i(t-s) \xi^{2}}\left[e^{i x \xi}+e^{-i x \xi}\right] \underline{e} \frac{\tilde{\mathcal{F}}\left(\begin{array}{c}
\phi \\
\psi
\end{array}\right)(\xi)}{\xi} d \xi
$$

50 The contribution of 5.8 is handled similarly. 
for the right hand factor $\tilde{\mathcal{F}}[\ldots]$ in 5.5 . We now use the expansion 5.3. Specifically, write

$$
\begin{aligned}
\tilde{U}^{(t)}(s, \cdot)= & e^{i\left(\Psi-\Psi_{\infty}\right)_{1}(s)-i\left(\Psi-\Psi_{\infty}\right)_{1}(t)} \sum_{j} \alpha_{j} \tilde{U}_{j, \mathrm{dis}}\left(s, .-\lambda_{\infty}\left(\mu-\mu_{\infty}\right)\right) \\
& +\sum_{k=1}^{6} \lambda_{k, j}(s) \eta_{k, \text { proper }}\left(.-\lambda_{\infty}\left(\mu-\mu_{\infty}\right)\right) .
\end{aligned}
$$

Here $\tilde{U}_{j \text {,dis }}$ is the first entry of the dispersive component of a tuple $\Gamma_{T, j}$ obtained upon applying $T_{A, \lambda, \beta, \omega, \gamma, \mu}$, and $\lambda_{k, j}$ are the corresponding root components. The contribution of the root part here is again easy to control due to the strong local decay estimate, and hence omitted. We may then assume, recalling (3.27), that each of the $\tilde{U}_{j \text {,dis }}$ satisfies a schematic equation of the form

$$
\begin{aligned}
\left(-i \partial_{s}+\right. & \Delta) \partial_{x} \overline{e^{i s} \tilde{U}_{j, \mathrm{dis}}^{(t)}}(s, \cdot)=e^{-i s} V_{j} \tilde{U}_{j, \mathrm{dis}}^{(t)}(s, \cdot)+e^{-i s} V_{j} \overline{\tilde{U}_{j, \mathrm{dis}}^{(t)}}(s, \cdot) \\
& +e^{-i s} V_{j} \partial_{x} \tilde{U}_{j, \mathrm{dis}}^{(t)}(s, \cdot)+e^{-i s} V_{j} \partial_{x} \overline{\tilde{U}_{j, \mathrm{dis}}^{(t)}}(s, \cdot)+\cdots+\partial_{x}\left[\left|\tilde{U}_{j}^{(t)}\right|^{4} \overline{e^{i s} \tilde{U}_{j}^{(t)}}\right]_{\mathrm{dis}}(s, \cdot) .
\end{aligned}
$$

Here $V_{j}$ denotes certain Schwartz functions whose fine structure beyond uniform bounds is irrelevant. We can then write

$$
\begin{aligned}
& \partial_{x} \overline{\tilde{U}_{j, \mathrm{dis}}}(s, x) \\
& \quad=\int_{-\infty}^{\infty} \int_{0}^{s} \frac{e^{i(s-\lambda)}}{\sqrt{s-\lambda}} e^{-\frac{(x-y)^{2}}{i(s-\lambda)}}\left[V_{j} \tilde{U}_{j, \mathrm{dis}}^{(s)}(\lambda, y)+\partial_{x}\left[\left|\tilde{U}_{j}^{(s)}\right|^{4} \overline{\tilde{U}_{j}^{(s)}}(\lambda, \cdot)\right]_{\mathrm{dis}}\right] d \lambda d y+\cdots .
\end{aligned}
$$

It will now suffice to control the contribution of one of the $\tilde{U}_{j \text {,dis }}$. This is the most delicate part of the argument. We shall break the integrand inside (5.10) into a number of pieces each of which leads to a manageable contribution. We only do this for the terms displayed in 5.10), the other ones being handled similarly. Specifically, write

$$
\begin{aligned}
& \int_{-\infty}^{\infty} \int_{0}^{s} \frac{e^{i(s-\lambda)}}{\sqrt{s-\lambda}} e^{-\frac{(x-y)^{2}}{i(s-\lambda)}} V_{j} \tilde{U}_{j, \mathrm{dis}}^{(s)}(\lambda, y) d \lambda d y \\
&=\int_{-\infty}^{\infty} \int_{0}^{s} \frac{e^{i(s-\lambda)}}{\sqrt{s-\lambda}} e^{-\frac{(x-y)^{2}}{i(s-\lambda)}} \chi_{<s-s^{2 \epsilon}}(\lambda) \chi_{<s^{\epsilon}}(y) V_{j} \tilde{U}_{j, \mathrm{dis}}^{(s)}(\lambda, y) d \lambda d y \\
&+\int_{-\infty}^{\infty} \int_{0}^{s} \frac{e^{i(s-\lambda)}}{\sqrt{s-\lambda}} e^{-\frac{(x-y)^{2}}{i(s-\lambda)}} \chi_{<s-s^{2 \epsilon}}(\lambda) \chi_{\geq s^{\epsilon}}(y) V_{j} \tilde{U}_{j, \mathrm{dis}}^{(s)}(\lambda, y) d \lambda d y \\
&+\int_{-\infty}^{\infty} \int_{0}^{s} \frac{e^{i(s-\lambda)}}{\sqrt{s-\lambda}} e^{-\frac{(x-y)^{2}}{i(s-\lambda)}} \chi_{\geq s-s^{2 \epsilon}}(\lambda) V_{j} \tilde{U}_{j, \mathrm{dis}}^{(s)}(\lambda, y) d \lambda d y .
\end{aligned}
$$

The last two terms lead to acceptable contributions: note that

$$
\left\|\int_{-\infty}^{\infty} \int_{0}^{s} \frac{1}{\sqrt{s-\lambda}} e^{-\frac{(x-y)^{2}}{i(s-\lambda)}} \chi_{\geq s^{\epsilon}}(y) V_{j} \tilde{U}_{j, \mathrm{dis}}(\lambda, y) d \lambda d y\right\|_{L_{x}^{2}} \lesssim e^{-s^{\epsilon}},
$$




$$
\begin{aligned}
&\left\|\int_{-\infty}^{\infty} \int_{0}^{s} \frac{1}{\sqrt{s-\lambda}} e^{-\frac{(x-y)^{2}}{i(s-\lambda)}} \chi_{<s^{\epsilon}}(y) \chi_{\geq s-s^{2 \epsilon}}(\lambda) V_{j} \tilde{U}_{j, \mathrm{dis}}(\lambda, y) d \lambda d y\right\|_{L_{x}^{2}} \\
& \lesssim\langle s\rangle^{-3 / 2}\langle s\rangle^{2 \epsilon+\delta_{3}}
\end{aligned}
$$

If then, returning all the way to the beginning of the proof of this lemma, one substitutes the corresponding terms in the Duhamel formula for $\tilde{U}_{\text {dis }}, \overline{\tilde{U}}_{\text {dis }}$ directly for the fifth factors $\tilde{U}^{(t)}, \overline{\tilde{U}^{(t)}}$ (up to a phase factor and translation) in

$$
\int_{0}^{t / 2}\left\langle e^{i(t-s) \mathcal{H}}\left(\begin{array}{c}
\left|\tilde{U}^{(t)}\right|^{4} \tilde{U}^{(t)}(s, \cdot) \\
-\left|\tilde{U}^{(t)}\right|^{4} \overline{\tilde{U}^{(t)}}(s, \cdot)
\end{array}\right)_{\mathrm{dis}}, \phi\right\rangle d s,
$$

one easily bounds this contribution by $\lesssim\langle t\rangle^{-3 / 2}$. Similarly, we have

$$
\begin{array}{r}
\left\|\int_{-\infty}^{\infty} \int_{0}^{s} \frac{1}{\sqrt{s-\lambda}} e^{-\frac{(x-y)^{2}}{i(s-\lambda)}} \chi_{>s^{1 / 2}}(\lambda) \partial_{y}\left[\left|\tilde{U}_{j}^{(s)}\right|^{4}(\lambda, y) \tilde{U}_{j}^{(s)}(\lambda, y)\right] d \lambda d y\right\|_{L_{x}^{2}} \\
\lesssim\langle s\rangle^{-1 / 2+C \delta_{2}}
\end{array}
$$

which leads to a similar conclusion upon substituting this integral for the last factors $\tilde{U}^{(t)}$, $\overline{\tilde{U}^{(t)}}$. Finally, note that on account of the pseudo-conformal conservation law, for $x>\lambda$ we have

whence we can estimate

$$
\left\|\tilde{U}_{j}(\lambda, \cdot)\right\|_{L_{x}^{2}} \sim\left\|\frac{\lambda}{x} \nabla \tilde{U}_{j}(\lambda, \cdot)\right\|_{L_{x}^{2}},
$$

$$
\begin{aligned}
\left\|\int_{-\infty}^{\infty} \int_{0}^{s^{1 / 2}} \frac{1}{\sqrt{s-\lambda}} e^{-\frac{(x-y)^{2}}{i(s-\lambda)}}\left|\tilde{U}_{j}(\lambda, y)\right|^{4} \chi_{>s^{1 / 2}}(y) \tilde{U}_{j}(y, \lambda) d y\right\|_{L_{x}^{2}} \\
\\
\lesssim s^{-1 / 2} \int_{0}^{s^{1 / 2}} \lambda^{-2} \lambda d \lambda \lesssim\langle s\rangle^{-1 / 2} \log \langle s\rangle,
\end{aligned}
$$

and the argument proceeds as before. This discussion justifies us in substituting

$$
\begin{aligned}
\partial_{x} \tilde{U}_{j}(s, x)=\int_{-\infty}^{\infty} \int_{0}^{s} & \frac{1}{\sqrt{s-\lambda}} e^{-\frac{(x-y)^{2}}{i(s-\lambda)}}\left[\chi_{<s-s^{2 \epsilon}} \chi_{<s^{\epsilon}}(y) V_{j} \tilde{U}_{j}^{(s)}(\lambda, y)\right. \\
& \left.\quad+\chi_{<s^{1 / 2}}(y) \chi_{<s^{1 / 2}}(\lambda) \partial_{y}\left[\left|\tilde{U}_{j}^{(s)}\right|^{4} \overline{\tilde{U}_{j}^{(s)}}(\lambda, y)\right]\right] d \lambda d y+\cdots .
\end{aligned}
$$

Next, write as before

$$
\begin{aligned}
\int_{-\infty}^{0} e^{i(t-s) \xi^{2}} \chi_{<t} 1000(\xi)\left[e^{i x \xi}+e^{-i x \xi}\right] \underline{e} \frac{\tilde{\mathcal{F}}\left(\begin{array}{c}
\phi \\
\psi
\end{array}\right)(\xi)}{\xi} d \xi & \\
& =\frac{1}{\sqrt{t-s}} \int_{-\infty}^{\infty} e^{-\frac{\left(x-y^{\prime}\right)^{2}}{i(t-s)}} \tilde{g}\left(y^{\prime}\right) d y^{\prime}
\end{aligned}
$$

where

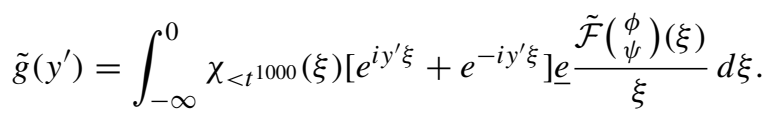


We observe that we may include a cutoff $\chi_{<(t-s)^{1 / 2}}\left(y^{\prime}\right)$ in front of $\tilde{g}\left(y^{\prime}\right)$; this is on account of the estimate $\left|\tilde{g}\left(y^{\prime}\right)\right| \lesssim y^{\prime-2} \log t$, and hence

$$
\left|\frac{1}{\sqrt{t-s}} \int_{-\infty}^{\infty} e^{-\frac{\left(x-y^{\prime}\right)^{2}}{i(t-s)}} \chi_{\geq(t-s)^{1 / 2}\left(y^{\prime}\right) \tilde{g}\left(y^{\prime}\right) d y^{\prime}}\right| \lesssim(t-s)^{-1} \log t,
$$

whence the corresponding contribution to 5.5 is acceptable. Now keeping in mind that our point of departure was the expression

$$
\int_{x}^{\infty} \chi>0\left(\begin{array}{c}
\overline{\tilde{U}^{(t)}}(s) \\
\tilde{U}^{(t)}(s)
\end{array}\right) \times e^{-i(t-s) \mathcal{H}^{*}}\left(\begin{array}{l}
\phi \\
\psi
\end{array}\right)_{\mathrm{dis}}(y) d y,
$$

which, amongst other terms, led to the expression

$$
\chi>0(x) s \partial_{x} \overline{\tilde{U}^{(t)}} \frac{e^{i(t-s)}}{t-s} \int_{-\infty}^{0} e^{i(t-s) \xi^{2}}\left[e^{i x \xi}+e^{-i x \xi}\right] \underline{e} \frac{\tilde{\mathcal{F}}\left(\begin{array}{l}
\phi \\
\psi
\end{array}\right)(\xi)}{\xi} d \xi,
$$

and substituting the expression (5.11) for $\partial_{x} \tilde{U}^{(t)}$ (we neglect here a phase shift as well as small translation in passing from $\tilde{U}$ to $\tilde{U}^{(t)}$, which do not affect the estimates), while also re-expressing the right hand integral in terms of $\tilde{g}$ and including the extra cutoff as above, we arrive at the following expressions:

$$
\begin{aligned}
& \frac{s}{(t-s)^{3 / 2}} \int_{x_{0}}^{\infty} \int_{-\infty}^{\infty} \int_{-\infty}^{\infty} \chi>0(x) \\
& \cdot \int_{0}^{s} \frac{1}{\sqrt{s-\lambda}} e^{-\frac{(x-y)^{2}}{i(s-\lambda)}} \chi_{<s^{\epsilon}}(y) \chi_{<s-s^{2 \epsilon}}(\lambda) V_{j} \tilde{U}_{j}^{(s)}(\lambda, y) e^{-\frac{\left(x-y^{\prime}\right)^{2}}{i(t-s)}}
\end{aligned}
$$

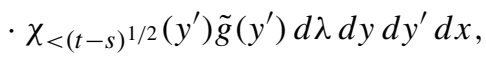

$$
\begin{aligned}
& \frac{s}{(t-s)^{3 / 2}} \int_{x_{0}}^{\infty} \int_{-\infty}^{\infty} \int_{-\infty}^{\infty} \chi>0(x) \\
& \cdot \int_{0}^{s} \frac{1}{\sqrt{s-\lambda}} e^{-\frac{(x-y)^{2}}{i(s-\lambda)}} \chi_{<s^{1 / 2}}(y) \chi_{<s^{1 / 2}}(\lambda) \partial_{y}\left[\left|\tilde{U}_{j}^{(s)}(\lambda, y)\right|^{4} \tilde{U}_{j}^{(s)}(y, \lambda)\right] e^{-\frac{\left(x-y^{\prime}\right)^{2}}{i(t-s)}}
\end{aligned}
$$

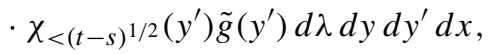

where $x_{0}$ ranges over $[0, \infty]$, plus similar terms which can be treated identically. Write

$$
e^{-\frac{(x-y)^{2}}{i(s-\lambda)}} e^{-\frac{\left(x-y^{\prime}\right)^{2}}{i(t-s)}}=e^{+i\left[\left(\frac{1}{s-\lambda}+\frac{1}{t-s}\right) x^{2}-\frac{2 x y}{s-\lambda}-\frac{2 x y^{\prime}}{t-s}\right]} e^{+\frac{i y^{2}}{s-\lambda}+\frac{i y^{\prime 2}}{t-s}} .
$$

This can be rewritten as

$$
e^{i\left(x \sqrt{\frac{1}{s-\lambda}+\frac{1}{t-s}}-y_{1}\right)^{2}} e^{i y_{2}}
$$

for certain functions $y_{1,2}\left(y, y^{\prime}, s, \lambda, t\right)$. Our restrictions in either term 5.12) or 5.13) ensure that $y_{1}=O(1)$. Carrying out the $x$-integration, we obtain

$$
\begin{aligned}
& \frac{1}{\sqrt{s-\lambda}} \int_{x_{0}}^{\infty} e^{i\left(x \sqrt{\frac{1}{s-\lambda}+\frac{1}{t-s}}-y_{1}\right)^{2}} d x \\
& \quad=\frac{1}{\sqrt{s-\lambda}}\left(\frac{1}{s-\lambda}+\frac{1}{t-s}\right)^{-1 / 2} S\left(x_{0} \sqrt{\frac{1}{s-\lambda}+\frac{1}{t-s}}-y_{1}\right)
\end{aligned}
$$


where $S(y)=\int_{y}^{\infty} e^{i x^{2}} d x=e^{i y^{2}} / y+O\left(y^{-2}\right)$ as $y \rightarrow+\infty$. Finally, we need to estimate

$\xi \int_{0}^{\infty}\left[e^{i x_{0} \xi}+e^{-i x_{0} \xi}\right] \frac{1}{\sqrt{s-\lambda}}\left(\frac{1}{s-\lambda}+\frac{1}{t-s}\right)^{-1 / 2} S\left(x_{0} \sqrt{\frac{1}{s-\lambda}+\frac{1}{t-s}}-y_{1}\right) d x_{0}$.

Here it is important that $y_{1}$ be uniformly bounded. The oscillatory nature of $S(y)$ allows us to bound this integral by

$$
\lesssim|\xi| \sqrt{\langle s\rangle} \lesssim\langle s\rangle^{-1 / 4}
$$

This bound suffices if one combines it with the estimates immediately after 5.5 to give the bound $(\log t)^{2}\langle t\rangle^{-3 / 2}$ for $(5.5)$. Note that the integrals in $y, y^{\prime}$ converge due to our estimates on $g(y), \tilde{g}\left(y^{\prime}\right)$.

Continuing in the situation $\xi \in(-\infty, 0]$, we proceed to the mixed frequency case. This is the expression

$$
\begin{aligned}
& \int_{-\infty}^{0} \int_{0}^{t / 2}\left\langle\left(\begin{array}{c}
P_{\geq a}\left[\chi_{>0}\left|\tilde{U}^{(t)}\right|^{2}(s)\right] P_{<a}\left[\left|\tilde{U}^{(t)}\right|^{2}(s)\right] \\
-P_{\geq a}\left[\chi_{>0}\left|\tilde{U}^{(t)}\right|^{2}(s)\right] P_{<a}\left[\left|\tilde{U}^{(t)}\right|^{2}(s)\right]
\end{array}\right),\left(e^{i x \xi}-e^{-i x \xi}\right) \underline{e}\right\rangle
\end{aligned}
$$

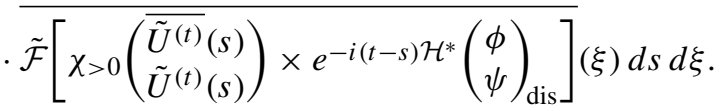

We shall be more terse here as the treatment mirrors that before. We first employ the ordinary Plancherel's Theorem to replace the $\xi$-integral (up to negligible error terms) by an expression

$$
\left\langle T_{z} P_{<a}\left[\left|\tilde{U}^{(t)}\right|^{2}(s)\right], T_{z} P_{\geq a}\left[\chi>0\left|\tilde{U}^{(t)}\right|^{2}(s)\right]\left\langle\underline{e}, \chi_{>0}\left(\begin{array}{c}
\overline{\tilde{U}^{(t)}}(s) \\
\tilde{U}^{(t)}(s)
\end{array}\right) \times e^{-i(t-s) \mathcal{H}^{*}}\left(\begin{array}{c}
\phi \\
\psi
\end{array}\right)_{\mathrm{dis}}\right\rangle\right\rangle,
$$

where we have to integrate over $z$ in the end which will cost $\log t$, as before. Express this in vectorial form as

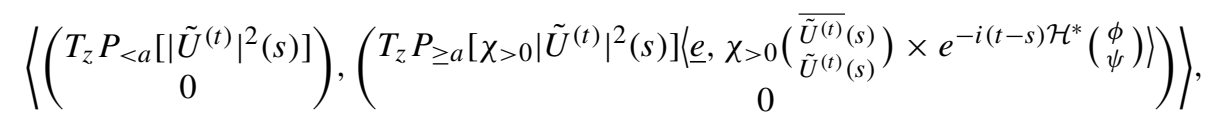

Decompose this into the following two terms:

$$
\begin{aligned}
& \left\langle\chi_{>0}(x)\left(\begin{array}{c}
T_{z} P_{<a}\left[\left|\tilde{U}^{(t)}\right|^{2}(s)\right] \\
0
\end{array}\right),\right.
\end{aligned}
$$

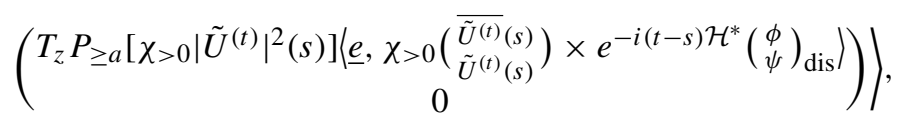

$$
\begin{aligned}
& \left\langle\chi_{<0}(x)\left(\begin{array}{c}
T_{z} P_{<a}\left[\left|\tilde{U}^{(t)}\right|^{2}(s)\right] \\
0
\end{array}\right),\right. \\
& \left.\left(\begin{array}{c}
T_{z} P_{\geq a}\left[\chi>0\left|\tilde{U}^{(t)}\right|^{2}(s)\right]\left\langle\underline{e}, \chi_{>0}\left(\begin{array}{c}
\overline{\tilde{U}^{(t)}}(s) \\
\tilde{U}^{(t)}(s)
\end{array}\right) \times e^{-i(t-s) \mathcal{H}^{*}}\left(\begin{array}{l}
\phi \\
\psi
\end{array}\right)_{\mathrm{dis}}\right) \\
0
\end{array}\right)\right\rangle .
\end{aligned}
$$


These being treated similarly, we treat the first term: commence by replacing $\chi_{>0}(x)$ - $\left(\begin{array}{c}T_{z} P_{<a}\left[\left|\tilde{U}^{(t)}\right|^{2}(s)\right] \\ 0\end{array}\right)$ by its dispersive part. This is done as in the mixed frequency case treated earlier. Then use the distorted Plancherel's Theorem 2.3, which produces

$$
\begin{aligned}
\int_{-\infty}^{\infty} & \frac{\mathcal{F}}{}\left[\chi_{>0}(x)\left(\begin{array}{c}
T_{z} P_{<a}\left[\left|\tilde{U}^{(t)}\right|^{2}(s)\right] \\
0
\end{array}\right)\right](\xi) \\
& \cdot \tilde{\mathcal{F}}\left[\left(\begin{array}{c}
T_{z} P_{\geq a}\left[\chi_{>0}\left|\tilde{U}^{(t)}\right|^{2}(s)\right]\left\langle\underline{e}, \chi_{>0}\left(\begin{array}{l}
\left(\tilde{U}^{(t)}(s)\right. \\
\tilde{U}^{(t)}(s)
\end{array}\right) \times e^{-i(t-s) \mathcal{H}^{*}}\left(\begin{array}{l}
\phi \\
\psi
\end{array}\right)\right. \\
0
\end{array}\right)\right](\xi) d \xi
\end{aligned}
$$

Divide this into the integral over $(-\infty, 0]$ as well as the integral over $[0, \infty)$. We treat the more difficult former case, the latter already having been dealt with. We recast this as

$$
\begin{aligned}
& \int_{-\infty}^{0}\left\langle\chi>0(x)\left(\begin{array}{c}
T_{z} P_{<a}\left[\left|\tilde{U}^{(t)}\right|^{2}(s)\right] \\
0
\end{array}\right),\left[e^{i x \xi}-e^{-i x \xi}+(1+r(-\xi)) e^{-i x \xi}\right] \underline{e}+\phi(x, \xi)\right\rangle
\end{aligned}
$$

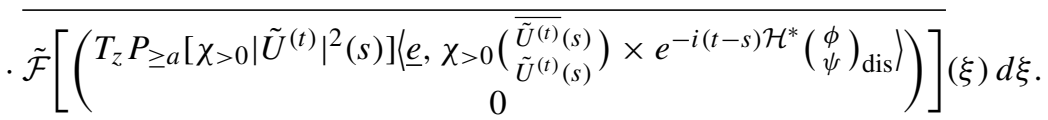

The contributions of $1+r(-\xi)$ and $\phi(x, \xi)$ are straightforward, and handled as before. We then need to estimate the following two contributions:

$$
\begin{aligned}
& \int_{-\infty}^{0}\left\langle P_{\geq a}\left[\chi>0(x)\left(\begin{array}{c}
T_{z} P_{<a}\left[\left|\tilde{U}^{(t)}\right|^{2}(s)\right] \\
0
\end{array}\right)\right],\left[e^{i x \xi}-e^{-i x \xi}\right] \underline{e}\right\rangle \\
& \overline{\tilde{\mathcal{F}}\left[\left(\begin{array}{c}
T_{z} P_{\geq a}\left[\chi_{>0}\left|\tilde{U}^{(t)}\right|^{2}(s)\right](\underline{e}, \chi>0 \\
0
\end{array}\left(\begin{array}{c}
\tilde{U}_{\tilde{U}^{(t)}(s)}^{(s)} \\
0
\end{array}\right) \times e^{\left.\left.\left.-i(t-s) \mathcal{H}^{*}\left(\begin{array}{l}
\phi \\
\psi
\end{array}\right)_{\mathrm{dis}}\right)\right)\right]}\right.\right.}(\xi) d \xi, \\
& \int_{-\infty}^{0}\left\langle P_{<a}\left[\chi>0(x)\left(\begin{array}{c}
T_{z} P_{<a}\left[\left|\tilde{U}^{(t)}\right|^{2}(s)\right] \\
0
\end{array}\right)\right],\left[e^{i x \xi}-e^{-i x \xi}\right] \underline{e}\right\rangle \\
& \overline{\mathcal{F}\left[\left(\begin{array}{c}
T_{z} P_{\geq a}\left[\chi>0\left|\tilde{U}^{(t)}\right|^{2}(s)\right]\left(\underline{e}, \chi_{>0}\left(\begin{array}{c}
\tilde{U}_{\tilde{U}^{(t)}(s)}^{(t)} \\
0
\end{array}\right) \times e^{-i(t-s) \mathcal{H}^{*}}\left(\begin{array}{l}
\phi \\
\psi
\end{array}\right)_{\mathrm{dis}}\right)
\end{array}\right)\right]}(\xi) d \xi .
\end{aligned}
$$

Consider the first of them: it is straightforward to replace $P_{\geq a}\left[\chi>0(x)\left(\begin{array}{c}T_{z} P_{<a}\left[\left|\tilde{U}^{(t)}\right|^{2}(s)\right] \\ 0\end{array}\right)\right]$ by $P_{\geq a}\left[\chi>0(x)\left(\begin{array}{c}T_{z}\left[\left|\tilde{U}^{(t)}\right|^{2}(s)\right] \\ 0\end{array}\right)\right]$, by arguing as for the high-high case. Then we replace this term by

$$
\begin{aligned}
& \int_{-\infty}^{0}\left\langle\partial_{x} \Delta^{-1} P_{\geq a} \partial_{x}\left[\chi>0(x)\left(\begin{array}{c}
T_{z} P_{<a}\left[\left|\tilde{U}^{(t)}\right|^{2}(s)\right] \\
0
\end{array}\right)\right],\left[e^{i x \xi}-e^{-i x \xi}\right] \underline{e}\right) \\
& \overline{\left.. \tilde{\mathcal{F}}\left[\left(\begin{array}{c}
T_{z} P_{\geq a}\left[\chi>0\left|\tilde{U}^{(t)}\right|^{2}(s)\right](\underline{e}, \chi>0 \\
0 \\
0
\end{array}\left(\begin{array}{c}
\tilde{U}^{(t)}(s) \\
\tilde{U}^{(t)}
\end{array}\right) \times e^{-i(t-s) \mathcal{H}^{*}}\left(\begin{array}{l}
\phi \\
\psi
\end{array}\right)_{\mathrm{dis}}\right)\right)\right]}(\xi) d \xi .
\end{aligned}
$$


First let the inner derivative $\partial_{x}$ fall onto the factor $\chi>0(x)$. This results in

$$
\begin{aligned}
& \int_{-\infty}^{0}\left\langle\partial_{x} \Delta^{-1} P_{\geq a}\left[\delta_{0}(x)\left(\begin{array}{c}
T_{z} P_{<a}\left[\left|\tilde{U}^{(t)}\right|^{2}(s)\right] \\
0
\end{array}\right)\right],\left[e^{i x \xi}-e^{-i x \xi}\right] \underline{e}\right\rangle
\end{aligned}
$$

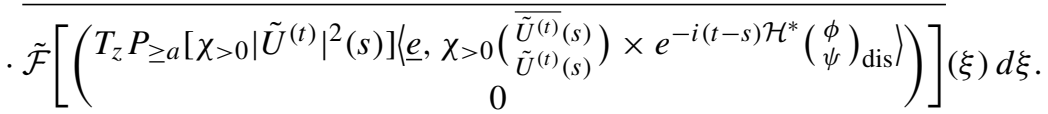

In order to estimate this, we decompose it further into two contributions:

$$
\begin{aligned}
& \int_{-\infty}^{0}\left\langle\partial_{x} \Delta^{-1} P_{\langle s\rangle^{-1 / 2} \geq . \geq a}\left[\delta_{0}(x)\left(\begin{array}{c}
T_{z} P_{<a}\left[\left|\tilde{U}^{(t)}\right|^{2}(s)\right] \\
0
\end{array}\right)\right],\left[e^{i x \xi}-e^{-i x \xi}\right] \underline{e}\right\rangle
\end{aligned}
$$

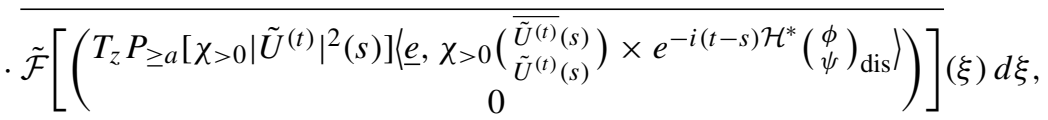

$$
\begin{aligned}
& \int_{-\infty}^{0}\left\langle\partial_{x} \Delta^{-1} P_{>\langle s\rangle^{-1 / 2}}\left[\delta_{0}(x)\left(\begin{array}{c}
T_{z} P_{<a}\left[\left|\tilde{U}^{(t)}\right|^{2}(s)\right] \\
0
\end{array}\right)\right],\left[e^{i x \xi}-e^{-i x \xi}\right] \underline{e}\right\rangle \\
& \overline{\tilde{\mathcal{F}}\left[\left(\begin{array}{c}
T_{z} P_{\geq a}\left[\chi_{>0}\left|\tilde{U}^{(t)}\right|^{2}(s)\right]\left\langle\underline{e}, \chi_{>0}\left(\begin{array}{c}
\tilde{U}_{\tilde{U}^{(t)}}^{(t)}(s) \\
0
\end{array}\right) \times e^{-i(t-s) \mathcal{H}^{*}}\left(\begin{array}{l}
\phi \\
\psi
\end{array}\right)_{\mathrm{dis}}\right)
\end{array}\right)\right]}(\xi) d \xi .
\end{aligned}
$$

Freeze the frequency of $P_{\langle s\rangle^{-1 / 2} \geq \cdot \geq a}\left[\delta_{0}(x)\left({ }_{T_{z} P_{<a}\left[\left|\tilde{U}^{(t)}\right|^{2}(s)\right]}^{0}\right)\right]$ to dyadic size $\sim b$. By Bernstein's inequality we get

$$
\left\|\Delta^{-1} \partial_{x} P_{b}\left[\delta_{0}(x)\left(\begin{array}{c}
T_{z} P_{<a}\left[\left|\tilde{U}^{(t)}\right|^{2}(s)\right] \\
0
\end{array}\right)\right]\right\|_{L_{x}^{2}} \lesssim b^{-1 / 2}\langle s\rangle^{-1+\epsilon\left(\delta_{2}\right)} .
$$

Next, note that

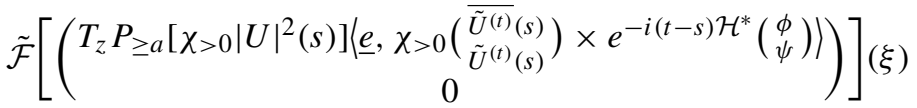

$$
\begin{aligned}
& =\int_{0}^{\infty}\left\langle\overline{\left[\left(e^{i x \xi}-e^{-i x \xi}+\left(1+r(-\xi) e^{-i x \xi}\right)\right) \underline{e}+\phi(x, \xi)\right]},\right.
\end{aligned}
$$

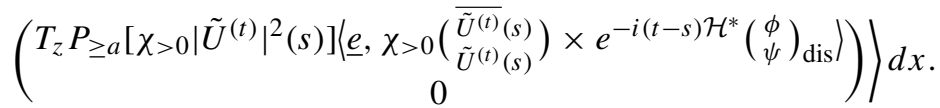

We treat here the most difficult contribution which comes as before from $e^{i x \xi}-e^{-i x \xi}$. Carrying out an integration by parts, we have to estimate the following terms:

$$
\begin{aligned}
& \int_{0}^{\infty} \xi\left[e^{i x \xi}+e^{-i x \xi}\right] \\
& \cdot\left(\begin{array}{c}
T_{z} P_{\geq a}\left[\chi>0\left|\tilde{U}^{(t)}\right|^{2}(s)\right] \int_{x}^{\infty}\left\langle\underline{e}, \chi>0\left(\begin{array}{c}
\overline{\tilde{U}^{(t)}}(s) \\
\tilde{U}^{(t)}(s)
\end{array}\right) \times e^{-i(t-s) \mathcal{H}^{*}}\left(\begin{array}{l}
\phi \\
\psi
\end{array}\right)_{\mathrm{dis}}(s, y)\right\rangle d y \\
0
\end{array}\right) d x,
\end{aligned}
$$




$$
\begin{aligned}
& \int_{0}^{\infty}\left[e^{i x \xi}-e^{-i x \xi}\right]
\end{aligned}
$$

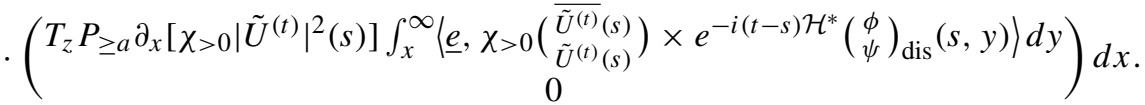

Our calculations for the low-low case above have taught us that we may assume 51

$$
\left|\int_{x}^{\infty}\left\langle\underline{e}, \chi>0\left(\begin{array}{c}
\overline{\tilde{U}^{(t)}}(s, y) \\
\tilde{U}^{(t)}(s, y)
\end{array}\right) \times e^{-i(t-s) \mathcal{H}^{*}}\left(\begin{array}{c}
\phi \\
\psi
\end{array}\right)_{\mathrm{dis}}\right\rangle d y\right| \lesssim\langle s\rangle\langle t-s\rangle^{-3 / 2} .
$$

Since $|\xi| \sim b$, the ordinary Plancherel's Theorem then implies that

$$
\begin{aligned}
& \| \int_{0}^{\infty} \xi\left[e^{i x \xi}+e^{-i x \xi}\right] \\
& \cdot\left(\begin{array}{c}
T_{z} P_{\geq a}\left[\chi_{>0}\left|\tilde{U}^{(t)}\right|^{2}(s)\right] \int_{x}^{\infty}\left\langle\underline{e}, \chi_{>0}\left(\begin{array}{c}
\left(\begin{array}{c}
\tilde{U}^{(t)}(s) \\
\tilde{U}^{(t)}(s)
\end{array}\right) \\
0
\end{array}\right) \times e^{-i(t-s) \mathcal{H}^{*}}\left(\begin{array}{l}
\phi \\
\psi
\end{array}\right)_{\mathrm{dis}}(s, y)\right\rangle d y
\end{array}\right) d x \|_{L_{\xi}^{2}} \\
& \lesssim\langle s\rangle\langle t-s\rangle^{-3 / 2} b\left\|T_{z} P_{\geq a}\left[\chi_{>0}\left|\tilde{U}^{(t)}\right|^{2}(s)\right]\right\|_{L_{x}^{2}} \lesssim b\langle s\rangle^{-3 / 4}\langle s\rangle\langle t-s\rangle^{-3 / 2} .
\end{aligned}
$$

The contribution of the term

$$
\begin{aligned}
& \int_{-\infty}^{0}\left[e^{i x \xi}-e^{-i x \xi}\right] \\
& \cdot\left(\begin{array}{c}
T_{z} P_{\geq a} \partial_{x}\left[\chi_{>0}\left|\tilde{U}^{(t)}\right|^{2}(s)\right] \int_{x}^{\infty}\left\langle\underline{e}, \chi_{>0}\left(\begin{array}{c}
\left(\begin{array}{c}
\tilde{U}^{(t)} \\
\tilde{U}^{(t)}(s)
\end{array}\right) \\
0
\end{array}\right) \times e^{-i(t-s) \mathcal{H}^{*}}\left(\begin{array}{l}
\phi \\
\psi
\end{array}\right)_{\mathrm{dis}}(s, y)\right\rangle d y
\end{array}\right) d x
\end{aligned}
$$

is handled similarly, by arguing as in the high-high case, using (5.4). Combining this with the bound (5.14), we estimate

$$
\begin{aligned}
& \mid \int_{-\infty}^{0}\left\langle\partial_{x} \Delta^{-1} P_{b}\left[\delta_{0}(x)\left(\begin{array}{c}
T_{z} P_{<a}\left[\left|\tilde{U}^{(t)}\right|^{2}(s)\right] \\
0
\end{array}\right)\right],\left[e^{i x \xi}-e^{-i x \xi}\right] \underline{e}\right\rangle
\end{aligned}
$$

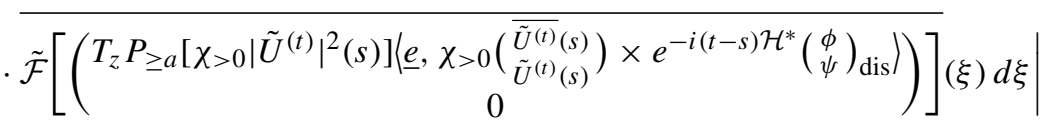

$$
\begin{aligned}
& \lesssim b^{-1 / 2}\langle s\rangle^{-1+\epsilon\left(\delta_{2}\right)} b\langle s\rangle^{1 / 4}\langle t-s\rangle^{-3 / 2} .
\end{aligned}
$$

Summing over all dyadic $b$ with $a<b<\langle s\rangle^{-1 / 2}$ and integrating over $s$ results in the bound $\lesssim\langle t\rangle^{-3 / 2+\delta_{3}}$. Next, we consider the contribution of

$$
\begin{aligned}
& \int_{-\infty}^{0}\left\langle\partial_{x} \Delta^{-1} P_{>\langle s\rangle^{-1 / 2}}\left[\delta_{0}(x)\left(\begin{array}{c}
T_{z} P_{<a}\left[\left|\tilde{U}^{(t)}\right|^{2}(s)\right] \\
0
\end{array}\right)\right],\left[e^{i x \xi}-e^{-i x \xi}\right] \underline{e}\right\rangle
\end{aligned}
$$

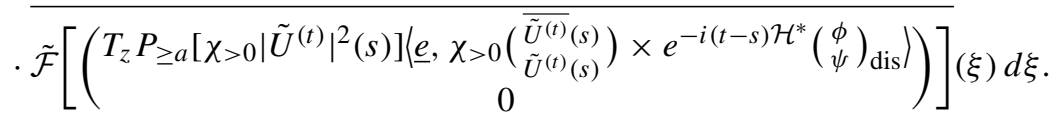

51 More precisely, we may write $\left(\frac{\tilde{U}^{(t)}}{\tilde{U}^{(t)}}\right)$ as the sum of two functions, one of which leads to a trivially estimable contribution, while the other satisfies the above inequality. 
We may again essentially replace $\tilde{\mathcal{F}}$ by the ordinary Fourier transform, and invoke the ordinary Plancherel's Theorem to replace this by (up to negligible errors)

$$
\begin{aligned}
& \left\langle\partial_{x} \Delta^{-1} P_{>\langle s\rangle^{-1 / 2}}\left[\delta_{0}(x)\left(\begin{array}{c}
T_{z} P_{<a}\left[\left|\tilde{U}^{(t)}\right|^{2}(s)\right] \\
0
\end{array}\right)\right],\right.
\end{aligned}
$$

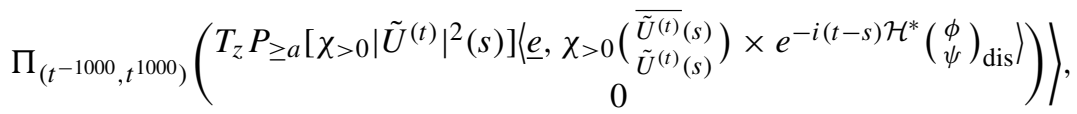

where $\Pi_{\left(t^{-1000}, t^{1000}\right)}$ is as in the discussion of the high-high case. We bound this by

$$
\begin{gathered}
\lesssim\left\|\partial_{x} \Delta^{-1} P_{>\langle s\rangle^{-1 / 2}}\left[\delta_{0}(x)\left(\begin{array}{c}
T_{z} P_{<a}\left[\left|\tilde{U}^{(t)}\right|^{2}(s)\right] \\
0
\end{array}\right)\right]\right\|_{L_{x}^{1}} \\
\cdot\left\|\Pi_{\left(t^{-1000}, t^{1000}\right)}\left(\begin{array}{c}
T_{z} P_{\geq a}\left[\chi>0\left|\tilde{U}^{(t)}\right|^{2}(s)\right](\underline{e}, \chi>0 \\
\left.\left.0 \begin{array}{c}
\tilde{\tilde{U}}^{(t)}(s) \\
\tilde{U}^{(t)}(s)
\end{array}\right) \times e^{-i(t-s) \mathcal{H}^{*}}\left(\begin{array}{c}
\phi \\
\psi
\end{array}\right)_{\mathrm{dis}}\right\rangle
\end{array}\right)\right\|_{L_{x}^{\infty}} .
\end{gathered}
$$

We can bound the preceding expression by

$$
\lesssim \log t \cdot\langle s\rangle^{\epsilon\left(\delta_{2}\right)}\langle s\rangle^{1 / 2}\langle s\rangle^{-1}\langle s\rangle^{-1}\langle s\rangle^{1 / 2}\langle t-s\rangle^{-3 / 2},
$$

which upon integration over $s$ leads to an acceptable bound. Thus in order to complete the discussion for the case $s<t / 2$, we need to estimate the expression

$$
\begin{aligned}
& \int_{-\infty}^{0}\left\langle P_{<a}\left[\chi_{>0}(x)\left(\begin{array}{c}
T_{z} P_{<a}\left[\left|\tilde{U}^{(t)}\right|^{2}(s)\right] \\
0
\end{array}\right)\right],\left[e^{i x \xi}-e^{-i x \xi}\right] \underline{e}\right\rangle
\end{aligned}
$$

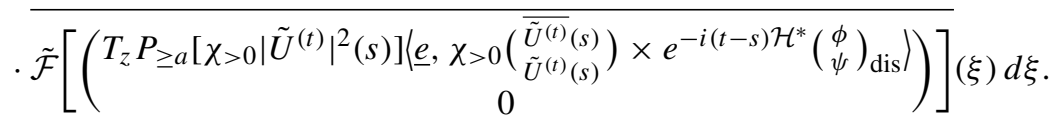

Keep in mind that we put $a=\langle s\rangle^{-3 / 4}$. As usual we simplify $\tilde{\mathcal{F}}[\ldots]$ and carry out an integration by parts, replacing this by

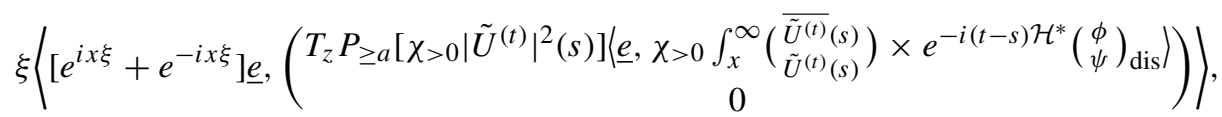

$$
\begin{aligned}
& \left\langle\left[e^{i x \xi}-e^{-i x \xi}\right] \underline{e},\left(\begin{array}{c}
T_{z} P_{\geq a} \partial_{x}\left[\chi>0\left|\tilde{U}^{(t)}\right|^{2}(s)\right]\left\langle\underline{e}, \chi_{>0} \int_{x}^{\infty}\left(\begin{array}{c}
\overline{\tilde{U}^{(t)}}(s) \\
\tilde{U}^{(t)}(s)
\end{array}\right) \times e^{-i(t-s) \mathcal{H}^{*}}\left(\begin{array}{c}
\phi \\
\psi
\end{array}\right)_{\mathrm{dis}}\right)
\end{array}\right)\right\rangle .
\end{aligned}
$$

Consider the first of these terms. The second is treated similarly, using (5.4). We have

$$
\begin{aligned}
& \| \chi_{<\langle s\rangle^{-3 / 4}}(\xi) \xi\left\langle\left[e^{i x \xi}+e^{-i x \xi}\right] \underline{e}\right.
\end{aligned}
$$

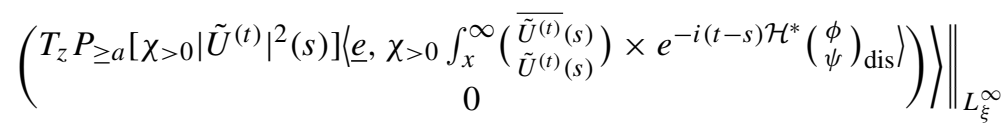

$$
\begin{aligned}
& \lesssim\langle s\rangle^{-3 / 4}\left\|T_{z} P_{\geq a}\left[\chi>0\left|\tilde{U}^{(t)}\right|^{2}(s)\right]\right\|_{L_{x}^{2}}\left\|\chi>0(x) \int_{x}^{\infty}\left(\begin{array}{c}
\overline{\tilde{U}^{(t)}}(s) \\
\tilde{U}^{(t)}(s)
\end{array}\right) \times e^{-i(t-s) \mathcal{H}^{*}}\left(\begin{array}{c}
\phi \\
\psi
\end{array}\right)_{\operatorname{dis}}\right\|_{L_{x}^{2}} .
\end{aligned}
$$


From our treatment of the low-low case we may assume that

$$
\left\|\chi>0(x) \int_{x}^{\infty}\left(\begin{array}{c}
\overline{\tilde{U}^{(t)}}(s) \\
\tilde{U}^{(t)}(s)
\end{array}\right) \times e^{-i(t-s) \mathcal{H}^{*}}\left(\begin{array}{c}
\phi \\
\psi
\end{array}\right)_{\text {dis }}\right\|_{L_{x}^{2}} \lesssim\langle s\rangle\langle s\rangle^{1 / 4}\langle t-s\rangle^{-3 / 2} .
$$

More precisely, we may decompose $\left(\frac{\tilde{U}^{(t)}}{\tilde{U}^{(t)}}\right)$ into two constituents one of which upon substitution into the original quintilinear expression immediately yields the desired estimate, while the other constituent satisfies the above estimate (see the discussion of the low-low case). We also have

$$
\left\|T_{z} P_{\geq a}\left[\chi>0\left|\tilde{U}^{(t)}\right|^{2}(s)\right]\right\|_{L_{x}^{2}} \lesssim a^{-1}\langle s\rangle^{-3 / 2} \lesssim\langle s\rangle^{-3 / 4} .
$$

Combining this with

$$
\left\|\left\langle P_{<a}\left[\chi>0(x)\left(\begin{array}{c}
T_{z} P_{<a}\left[\left|\tilde{U}^{(t)}\right|^{2}(s)\right] \\
0
\end{array}\right)\right],\left[e^{i x \xi}-e^{-i x \xi}\right] \underline{e}\right)\right\|_{L_{\xi}^{1}} \lesssim\langle s\rangle^{-3 / 4},
$$

we can bound

$$
\begin{aligned}
& \mid \int_{-\infty}^{0}\left\langle P_{<a}\left[\chi_{>0}(x)\left(\begin{array}{c}
T_{z} P_{<a}\left[\left|\tilde{U}^{(t)}\right|^{2}(s)\right] \\
0
\end{array}\right)\right],\left[e^{i x \xi}-e^{-i x \xi}\right] \underline{e}\right\rangle \\
& \cdot \overline{\xi\left\langle e^{i x \xi}+e^{-i x \xi},\left[\left(\begin{array}{c}
T_{z} P_{\geq a}\left[\chi_{>0}\left|\tilde{U}^{(t)}\right|^{2}(s)\right] \\
\left.\underline{e}, \chi_{>0} \int_{x}^{\infty}\left(\begin{array}{c}
\tilde{U}_{\tilde{U}^{(t)}}^{(t)}(s) \\
\tilde{U}^{(s)}
\end{array}\right) \times e^{-i(t-s) \mathcal{H}^{*}}\left(\begin{array}{c}
\phi \\
\psi
\end{array}\right)_{\mathrm{dis}}\right)
\end{array}\right)\right]\right\rangle} d \xi \mid \\
& \lesssim\langle s\rangle^{5 / 4}\langle s\rangle^{-3 / 4}\langle s\rangle^{-3 / 4}\langle s\rangle^{-3 / 4}\langle t-s\rangle^{-3 / 2} \text {, }
\end{aligned}
$$

which is again as desired upon integrating over $0 \leq s \leq t / 2$. This finally concludes treatment of Case A.

Case B: $s \geq t / 2$. The procedure here is basically identical to the preceding case A, so we shall be relatively short here: one divides into the cases

$$
\begin{gathered}
\int_{t / 2}^{t}\left\langle\left(\begin{array}{c}
\chi_{>0}\left|\tilde{U}^{(t)}\right|^{4} \tilde{U}^{(t)}(s, \cdot) \\
-\chi_{>0}\left|\tilde{U}^{(t)}\right|^{4} \tilde{\tilde{U}}^{(t)}(s, \cdot)
\end{array}\right), e^{-i(t-s) \mathcal{H}^{*}}\left(\begin{array}{c}
\phi \\
\psi
\end{array}\right)_{\mathrm{dis}}\right\rangle d s, \\
\int_{t / 2}^{t}\left\langle\left(\begin{array}{c}
\chi_{<0}\left|\tilde{U}^{(t)}\right|^{4} \tilde{U}^{(t)}(s, \cdot) \\
-\chi_{<0}\left|\tilde{U}^{(t)}\right|^{4} \tilde{\tilde{U}}^{(t)}(s, \cdot)
\end{array}\right), e^{-i(t-s) \mathcal{H}^{*}}\left(\begin{array}{c}
\phi \\
\psi
\end{array}\right)_{\mathrm{dis}}\right\rangle d s .
\end{gathered}
$$

Both being treated similarly, we shall only consider the first term. We easily reduce $\left(\begin{array}{c}\chi_{>0}\left|\tilde{U}^{(t)}\right|^{4} \tilde{U}^{(t)}(s, \cdot) \\ -\chi_{>0}\left|\tilde{U}^{(t)}\right|^{4} \tilde{U}^{(t)}(s, \cdot)\end{array}\right)$ to its dispersive part: note that

$$
\left|\left\langle\left\langle\left(\begin{array}{c}
\chi>0\left|\tilde{U}^{(t)}\right|^{4} \tilde{U}^{(t)}(s, \cdot) \\
-\chi_{>0}\left|\tilde{U}^{(t)}\right|^{4} \tilde{U}^{(t)}(s, \cdot)
\end{array}\right), \xi_{k(j)}\right\rangle \eta_{j}, e^{-i(t-s) \mathcal{H}^{*}}\left(\begin{array}{c}
\phi \\
\psi
\end{array}\right)_{\mathrm{dis}}\right\rangle\right| \lesssim s^{-6+\epsilon}(t-s)^{-3 / 2},
$$


which is significantly better than what we need. Now use the distorted Plancherel's Theorem to rewrite what remains as

$$
\sum_{ \pm} \int_{-\infty}^{\infty} \int_{t / 2}^{t} \mathcal{F}_{ \pm}\left(\begin{array}{c}
\chi_{>0}\left|\tilde{U}^{(t)}\right|^{4} \\
-\chi_{>0}\left|\tilde{U}^{(t)}\right|^{4}
\end{array}\right)(\xi) \overline{\left.\tilde{\mathcal{F}}_{ \pm}\left[\begin{array}{c}
\overline{\tilde{U}^{(t)}} \\
\tilde{U}^{(t)}
\end{array}\right) \times e^{-i(t-s) \mathcal{H}^{*}}\left(\begin{array}{c}
\phi \\
\psi
\end{array}\right)_{\mathrm{dis}}\right]}(\xi) d s d \xi
$$

We consider here the case + and $\xi \in[0, \infty)$ and how one has to modify the argument in case A to get the desired estimate. Analogous modifications will then also give the result for $\xi \in(-\infty, 0]$. Write (leaving out the subscript)

$$
\begin{aligned}
& \int_{0}^{\infty} \int_{t / 2}^{t} \mathcal{F}\left(\begin{array}{c}
\chi_{>0}\left|\tilde{U}^{(t)}\right|^{4} \\
-\chi_{>0}\left|\tilde{U}^{(t)}\right|^{4}
\end{array}\right)(\xi) \overline{\tilde{\mathcal{F}}\left[\left(\begin{array}{c}
\overline{\tilde{U}^{(t)}} \\
\tilde{U}^{(t)}
\end{array}\right) \times e^{-i(t-s) \mathcal{H}^{*}}\left(\begin{array}{c}
\phi \\
\psi
\end{array}\right)_{\mathrm{dis}}\right]}(\xi) d s d \xi \\
& \left.=\int_{0}^{\infty} \int_{t / 2}^{t}\left\langle\left(\begin{array}{c}
\chi>0\left|\tilde{U}^{(t)}\right|^{4} \\
-\chi>0 \mid
\end{array}\right),\left.s(\xi) \tilde{U}^{(t)}\right|^{4}\right)+\sigma_{3} \phi(x, \xi)\right\rangle \\
& \overline{\tilde{\mathcal{F}}\left[\left(\begin{array}{c}
\tilde{U}^{(t)} \\
\tilde{U}^{(t)}
\end{array}\right) \times e^{-i(t-s)} \mathcal{H}^{*}\left(\begin{array}{c}
\phi \\
\psi
\end{array}\right)_{\mathrm{dis}}\right]}(\xi) d s d \xi .
\end{aligned}
$$

The contribution of the local term $\phi(x, \xi)$ is again easy to handle. As usual, invoke the decomposition

$$
\begin{aligned}
& \int_{0}^{\infty} \int_{t / 2}^{t}\left\langle\left(\begin{array}{c}
\chi_{>0}\left|\tilde{U}^{(t)}\right|^{4} \\
-\chi_{>0}\left|\tilde{U}^{(t)}\right|^{4}
\end{array}\right), s(\xi) e^{i x \xi} \overline{\left.\overline{\mathcal{F}}\left[\begin{array}{c}
\left(\overline{\tilde{U}^{(t)}}\right. \\
\tilde{U}^{(t)}
\end{array}\right) \times e^{-i(t-s) \mathcal{H}^{*}}\left(\begin{array}{c}
\phi \\
\psi
\end{array}\right)_{\mathrm{dis}}\right](\xi)} d s d \xi\right. \\
& =\int_{0}^{\infty} \int_{t / 2}^{t}\left\langle\left(\begin{array}{c}
P_{\geq a}\left[\chi_{>0}\left|\tilde{U}^{(t)}\right|^{2}\right] P_{\geq a}\left[\left|\tilde{U}^{(t)}\right|^{2}\right] \\
-P_{\geq a}\left[\chi_{>0}\left|\tilde{U}^{(t)}\right|^{2}\right] P_{\geq a}\left[\left|\tilde{U}^{(t)}\right|^{2}\right]
\end{array}\right), s(\xi) e^{i x \xi}\right\rangle
\end{aligned}
$$

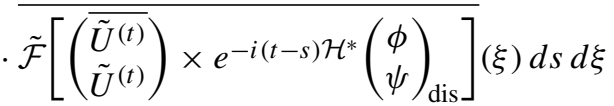

$$
\begin{aligned}
& +\int_{0}^{\infty} \int_{t / 2}^{t}\left\langle\left(\begin{array}{c}
P_{\geq a}\left[\chi_{>0}\left|\tilde{U}^{(t)}\right|^{2}\right] P_{<a}\left[\left|\tilde{U}^{(t)}\right|^{2}\right] \\
-P_{\geq a}\left[\chi_{>0}\left|\tilde{U}^{(t)}\right|^{2}\right] P_{<a}\left[\left|\tilde{U}^{(t)}\right|^{2}\right]
\end{array}\right), s(\xi) e^{i x \xi}\right\rangle
\end{aligned}
$$

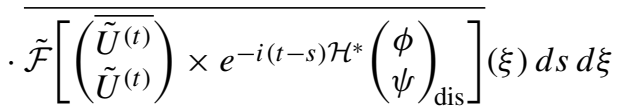

$$
\begin{aligned}
& +\int_{0}^{\infty} \int_{t / 2}^{t}\left\langle\left(\begin{array}{c}
P_{<a}\left[\chi_{>0}\left|\tilde{U}^{(t)}\right|^{2}\right] P_{\geq a}\left[\left|\tilde{U}^{(t)}\right|^{2}\right] \\
-P_{<a}\left[\chi_{>0}\left|\tilde{U}^{(t)}\right|^{2}\right] P_{\geq a}\left[\left|\tilde{U}^{(t)}\right|^{2}\right]
\end{array}\right), s(\xi) e^{i x \xi}\right\rangle
\end{aligned}
$$

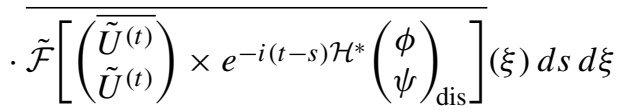

$$
\begin{aligned}
& +\int_{0}^{\infty} \int_{t / 2}^{t}\left\langle\left(\begin{array}{c}
P_{<a}\left[\chi_{>0}\left|\tilde{U}^{(t)}\right|^{2}\right] P_{<a}\left[\left|\tilde{U}^{(t)}\right|^{2}\right] \\
-P_{<a}\left[\chi_{>0}\left|\tilde{U}^{(t)}\right|^{2}\right] P_{<a}\left[\left|\tilde{U}^{(t)}\right|^{2}\right]
\end{array}\right), s(\xi) e^{i x \xi}\right\rangle \\
& \overline{\tilde{\mathcal{F}}\left[\left(\begin{array}{c}
\tilde{U}^{(t)} \\
\tilde{U}^{(t)}
\end{array}\right) \times e^{-i(t-s) \mathcal{H}^{*}}\left(\begin{array}{l}
\phi \\
\psi
\end{array}\right)_{\mathrm{dis}}\right]}(\xi) d s d \xi .
\end{aligned}
$$


We consider here the first term. Use the fact that for $\xi>0$ we have

$$
\begin{aligned}
\tilde{\mathcal{F}}\left[\chi_{>0}(x)\left(\begin{array}{c}
\overline{\tilde{U}^{(t)}} \\
\tilde{U}^{(t)}
\end{array}\right) \times e^{-i(t-s) \mathcal{H}^{*}}\left(\begin{array}{c}
\phi \\
\psi
\end{array}\right)_{\mathrm{dis}}\right](\xi) \\
\quad=\left\langle\chi_{>0}(x)\left(\begin{array}{c}
\overline{\tilde{U}^{(t)}} \\
\tilde{U}^{(t)}
\end{array}\right) \times e^{-i(t-s) \mathcal{H}^{*}}\left(\begin{array}{c}
\phi \\
\psi
\end{array}\right)_{\mathrm{dis}}, s(\xi) e^{i x \xi}+\phi(x, \xi)\right\rangle .
\end{aligned}
$$

The contribution from $\phi(x, \xi)$ is easy to handle: note that

$$
\left\|\left\langle\sigma_{3} \phi(x, \xi), \chi_{>0}(x)\left(\begin{array}{c}
\overline{\tilde{U}^{(t)}} \\
\tilde{U}^{(t)}
\end{array}\right) \times e^{-i(t-s) \mathcal{H}^{*}}\left(\begin{array}{l}
\phi \\
\psi
\end{array}\right)_{\mathrm{dis}}\right\rangle\right\|_{L_{\xi}^{2}} \lesssim\langle t-s\rangle^{-3 / 2} s^{-3 / 2+\epsilon},
$$

whence inserting into the full expressions and integrating over $s \geq t / 2$ results in the upper bound $\lesssim\langle T\rangle^{-5 / 2}$. Thus we now need to consider

$$
\begin{aligned}
& \int_{0}^{\infty} \int_{t / 2}^{t}\left\langle\left(\begin{array}{c}
P_{\geq a}\left[\chi_{>0}\left|\tilde{U}^{(t)}\right|^{2}\right] P_{\geq a}\left[\left|\tilde{U}^{(t)}\right|^{2}\right] \\
-P_{\geq a}\left[\chi_{>0}\left|\tilde{U}^{(t)}\right|^{2}\right] P_{\geq a}\left[\left|\tilde{U}^{(t)}\right|^{2}\right]
\end{array}\right), s(\xi) e^{i x \xi} \underline{e}\right\rangle \\
& \cdot \overline{\left\langle\chi>0(x)\left(\begin{array}{c}
\overline{\tilde{U}^{(t)}} \\
\tilde{U}^{(t)}
\end{array}\right) \times e^{-i(t-s) \mathcal{H}^{*}}\left(\begin{array}{c}
\phi \\
\psi
\end{array}\right)_{\mathrm{dis}}, s(\xi) e^{i x \xi} \underline{e}\right\rangle} d s d \xi .
\end{aligned}
$$

Using the ordinary Plancherel's Theorem, we replace this by (up to negligible error terms)

$$
\begin{aligned}
& \int_{0}^{\infty} \int_{t / 2}^{t}\left\langle P_{\geq a}\left[\chi>0\left|\tilde{U}^{(t)}\right|^{2}\right] P_{\geq a}\left[\left|\tilde{U}^{(t)}\right|^{2}\right],\right.\left.\Pi_{\left(t^{-1000}, t^{1000}\right)}\right\rangle \\
& \cdot\left\langle\underline{e}, \chi_{>0}(x)\left(\begin{array}{c}
\overline{\tilde{U}^{(t)}} \\
\tilde{U}^{(t)}
\end{array}\right) \times e^{-i(t-s) \mathcal{H}^{*}}\left(\begin{array}{c}
\phi \\
\psi
\end{array}\right)_{\mathrm{dis}}\right\rangle .
\end{aligned}
$$

This is all as in the case $s<t / 2$. At this point, though, we do not pull down the full power of $(t-s)^{-3 / 2}$, but only $(t-s)^{-1 / 2}$, which costs nothing in terms of weights. In other words, we estimate

$$
\left|\Pi_{\left(t^{-1000}, t^{1000}\right)}\left\langle\underline{\underline{e}}, \chi_{>0}(x)\left(\begin{array}{c}
\overline{\tilde{U}^{(t)}} \\
\tilde{U}^{(t)}
\end{array}\right) \times e^{-i(t-s) \mathcal{H}^{*}}\left(\begin{array}{c}
\phi \\
\psi
\end{array}\right)_{\mathrm{dis}}\right\rangle\right| \lesssim(t-s)^{-1 / 2} s^{-1 / 2} .
$$

Then use again the estimate

$$
\left\|P_{\geq a}\left[\chi_{>0}\left|\tilde{U}^{(t)}\right|^{2}\right] P_{\geq a}\left[\left|\tilde{U}^{(t)}\right|^{2}\right]\right\|_{L_{x}^{1}} \lesssim a^{-2} s^{-3} .
$$

Putting these together and integrating up over $s>t / 2$ yields the upper bound $\langle T\rangle^{-3 / 2}$ up to an arbitrarily small power error independent of $\delta_{3}$. The remaining terms above follow by similar modifications from the arguments for the case $s<t / 2$, and are omitted. This establishes the strong local dispersive estimate up to demonstrating the bound $\left\|C \partial_{x} \tilde{U}(s, \cdot)\right\|_{L_{x}^{2}} \lesssim s^{1 / 2+\epsilon\left(\delta_{2}\right)}$, which will be done in the next lemma. This finally concludes the proof of Lemma 5.7 
The next essential lemma improves the bound for the pseudo-conformal operator expression $C \tilde{U}_{\mathrm{dis}}^{\prime}$. Note again that the iterated structure of $A_{[0, T)}^{(n)}$ is not directly needed here, but only in order to invoke the already improved bound from the preceding lemma:

Lemma 5.8. Let $\Gamma_{T}^{\prime} \in A_{[0, T)}^{(2)}$. Then under the conditions of Theorem 4.5 and the assumption $\left|\int_{0}^{T} s^{2}\left\langle\left(\tilde{U}_{\mathrm{dis}}^{\prime(t)}\right)^{2}(s)-\left(\tilde{U}_{\mathrm{dis}}^{\prime(t)}\right)^{2}(s), \phi\right\rangle d s\right| \lesssim R^{2} \delta^{2}$ for $\phi \in \mathcal{A}$, we have

$$
\sup _{0 \leq t<T}\left\|C \tilde{U}_{\mathrm{dis}}^{\prime}(t, y)\right\|_{L_{y}^{2}} \leq \frac{R}{100} \delta .
$$

We also have the bound

$$
\sup _{0 \leq s \leq T}\left\|C \partial_{y} \tilde{U}_{\mathrm{dis}}^{\prime}\right\|_{L_{y}^{2}} \lesssim R \delta\langle s\rangle^{1 / 2}
$$

Proof. Recall the operator $C=y-2 s p$. Fix $0 \leq t<T$. We will derive a bound on $\sup _{0 \leq s<t}\left\|C \tilde{U}_{\text {dis }}^{\prime(t)}(s, y)\right\|_{L_{y}^{2}}$, which for $s=t$ implies the bound of the lemma. Now note

$$
\begin{aligned}
& i \partial_{s}\left\langle C_{1}\left(\frac{\tilde{U}_{\mathrm{dis}}^{\prime(t)}}{\tilde{U}_{\mathrm{dis}}^{\prime(t)}}\right),\left(\frac{\tilde{U}_{\mathrm{dis}}^{\prime(t)}}{\tilde{U}_{\mathrm{dis}}^{\prime(t)}}\right)\right\rangle \\
& \quad=\left\langle i \dot{C}_{1}\left(\frac{\tilde{U}_{\mathrm{dis}}^{\prime(t)}}{\tilde{U}_{\mathrm{dis}}^{\prime(t)}}\right)+i C_{1} \partial_{s}\left(\frac{\tilde{U}_{\mathrm{dis}}^{\prime(t)}}{\tilde{U}_{\mathrm{dis}}^{\prime(t)}}\right),\left(\frac{\tilde{U}_{\mathrm{dis}}^{\prime(t)}}{\tilde{U}_{\mathrm{dis}}^{\prime(t)}}\right)\right\rangle-\left\langle C_{1}\left(\frac{\tilde{U}_{\mathrm{dis}}^{\prime(t)}}{\tilde{U}_{\mathrm{dis}}^{\prime(t)}}\right), i \partial_{s}\left(\frac{\tilde{U}_{\mathrm{dis}}^{\prime(t)}}{\tilde{U}_{\mathrm{dis}}^{\prime(t)}}\right)\right\rangle,
\end{aligned}
$$

where

$$
C_{1}=\left(\begin{array}{cc}
(y-2 s p)^{2} & 0 \\
0 & (y+2 s p)^{2}
\end{array}\right), \quad p=-i \partial_{y}
$$

Then write the equation for $\left(\frac{\tilde{U}^{\prime(t)}}{\tilde{U}^{\prime(t)}}\right)_{\text {dis }}$ schematically as follows, with $\mathcal{H}_{0}=\left(\begin{array}{cc}\partial_{y}^{2}-1 & 0 \\ 0 & 1-\partial_{y}^{2}\end{array}\right)$ :

$$
\begin{aligned}
& i \partial_{s}\left(\frac{\tilde{U}^{\prime(t)}}{\tilde{U}^{\prime(t)}}\right)_{\mathrm{dis}}+\mathcal{H}_{0}\left(\frac{\tilde{U}^{\prime(t)}}{\tilde{U}^{\prime(t)}}\right)_{\mathrm{dis}} \\
& \quad=-\left[\left(\begin{array}{cc}
X & Y \\
Z & -X
\end{array}\right)\left(\frac{\tilde{U}^{(t)}}{\tilde{U}^{(t)}}\right)\right]_{\mathrm{dis}}-\left(\begin{array}{cc}
3 \phi_{0}^{4} & 2 \phi_{0}^{4} \\
-2 \phi_{0}^{4} & -3 \phi_{0}^{4}
\end{array}\right)\left(\frac{\tilde{U}^{\prime(t)}}{\tilde{U}^{\prime(t)}}\right)_{\mathrm{dis}}+\dot{\pi} \partial_{\pi} W+N\left(\tilde{U}^{(t)}, \pi\right)
\end{aligned}
$$

(cf. 3.27) $)$, where $\tilde{\phi}_{0}^{(t)}=\phi_{0}(v(t))\left(\cdot+\lambda_{\infty}\left[\mu-\mu_{\infty}\right](t)-\lambda_{\infty}\left[\mu-\mu_{\infty}\right](s)\right)$ and

$$
\begin{aligned}
X & =3\left[v^{2}\left(\tilde{\phi}_{0}^{(t)}\right)^{4}-\phi_{0}^{4}\right] \\
Y & =2 v^{2}\left(\tilde{\phi}_{0}^{(t)}\right)^{4} e^{2 i\left(\Psi-\Psi_{\infty}\right)(s)-2 i(\Psi-\Psi)_{1}(t)}-2 \phi_{0}^{4}(s), \\
Z & =-2 v^{2}\left(\tilde{\phi}_{0}^{(t)}\right)^{4} e^{-2 i\left(\Psi-\Psi_{\infty}\right)(s)+2 i\left(\Psi-\Psi_{\infty}\right)_{1}(t)}+2 \phi_{0}^{4}(s)
\end{aligned}
$$


If we substitute this back into the preceding and expand $\dot{C}_{1}$, we obtain the relation (here $v=v(s))$

$$
\begin{aligned}
& i \partial_{s}\left\langle C_{1}\left(\frac{\tilde{U}^{\prime(t)}}{\tilde{U}^{\prime(t)}}\right)_{\mathrm{dis}},\left(\frac{\tilde{U}^{\prime(t)}}{\tilde{U}^{\prime(t)}}\right)_{\mathrm{dis}}\right\rangle \\
& =\left\langle\left(\begin{array}{cc}
8 i s p^{2}-2-4 i y p & 0 \\
0 & 2+8 i s p^{2}+4 i y p
\end{array}\right)\left(\frac{\tilde{U}^{\prime(t)}}{\tilde{U}^{\prime(t)}}\right)_{\mathrm{dis}}+C_{1}\left(-\mathcal{H}_{0}\left(\frac{\tilde{U}^{\prime(t)}}{\tilde{U}^{\prime(t)}}\right)_{\mathrm{dis}}\right.\right. \\
& -\left[\left(\begin{array}{cc}
X & Y \\
Z & -X
\end{array}\right)\left(\frac{\tilde{U}^{(t)}}{\tilde{U}^{(t)}}\right)\right]_{\mathrm{dis}} \\
& \left.\left.-\left(\begin{array}{cc}
3 \phi_{0}^{4} & 2 \phi_{0}^{4} \\
-2 \phi_{0}^{4} & -3 \phi_{0}^{4}
\end{array}\right)\left(\frac{\tilde{U}^{\prime(t)}}{\tilde{U}^{\prime(t)}}\right)_{\mathrm{dis}}+\dot{\pi} \partial_{\pi} W+N\left(\tilde{U}^{(t)}, \pi\right)\right),\left(\frac{\tilde{U}^{\prime(t)}}{\tilde{U}^{\prime(t)}}\right)_{\mathrm{dis}}\right) \\
& +\left\langle C_{1}\left(\frac{\tilde{U}^{\prime(t)}}{\tilde{U}^{\prime(t)}}\right)_{\mathrm{dis}}, \mathcal{H}_{0}\left(\frac{\tilde{U}^{\prime(t)}}{\tilde{U}^{\prime(t)}}\right)_{\mathrm{dis}}\right. \\
& \left.+\left[\left(\begin{array}{cc}
X & Y \\
Z & -X
\end{array}\right)\left(\frac{\tilde{U}^{(t)}}{\tilde{U}^{(t)}}\right)\right]_{\mathrm{dis}}+\left(\begin{array}{cc}
3 \phi_{0}^{4} & 2 \phi_{0}^{4} \\
-2 \phi_{0}^{4} & -3 \phi_{0}^{4}
\end{array}\right)\left(\frac{\tilde{U}^{\prime(t)}}{\tilde{U}^{\prime(t)}}\right)_{\mathrm{dis}}-\dot{\pi} \partial_{\pi} W-N\left(\tilde{U}^{(t)}, \pi\right)\right) .
\end{aligned}
$$

We then observe that we may recast this complicated expression in the more manageable form

$$
\begin{aligned}
& \left\langle-C_{1}\left(\begin{array}{cc}
3 \phi_{0}^{4} & 2 \phi_{0}^{4} \\
-2 \phi_{0}^{4} & -3 \phi_{0}^{4}
\end{array}\right)\left(\frac{\tilde{U}^{\prime(t)}}{\tilde{U}^{\prime(t)}}\right)_{\mathrm{dis}}+\left(\begin{array}{cc}
3 \phi_{0}^{4} & -2 \phi_{0}^{4} \\
2 \phi_{0}^{4} & -3 \phi_{0}^{4}
\end{array}\right) C_{1}\left(\frac{\tilde{U}^{\prime(t)}}{\tilde{U}^{\prime(t)}}\right)_{\mathrm{dis}},\left(\frac{\tilde{U}^{\prime(t)}}{\tilde{U}^{\prime(t)}}\right)_{\mathrm{dis}}\right\rangle \\
& +\left\langle C_{1}\left(\dot{\pi} \partial_{\pi} W+N(\tilde{U}, \pi),\left(\frac{\tilde{U}^{\prime(t)}}{\tilde{U}^{\prime(t)}}\right)_{\mathrm{dis}}\right\rangle-\left\langle C_{1}\left(\frac{\tilde{U}^{\prime(t)}}{\tilde{U}^{\prime(t)}}\right)_{\mathrm{dis}}, \dot{\pi} \partial_{\pi} W+N(\tilde{U}, \pi)\right\rangle\right.
\end{aligned}
$$

modulo error terms which are absolutely integrable with respect to $s$. The last four terms in 5.15) shall be fairly straightforward to control. However, the first two appear to lead to a loss, as they are not absolutely integrable. Observe that we can be rewrite the sum of the first two terms as a commutator

$$
\left\langle\left[\left(\begin{array}{cc}
3 \phi_{0}^{4} & 2 \phi_{0}^{4} \\
2 \phi_{0}^{4} & 3 \phi_{0}^{4}
\end{array}\right),\left(\begin{array}{cc}
1 & 0 \\
0 & -1
\end{array}\right) C_{1}\right]\left(\frac{\tilde{U}^{\prime(t)}}{\tilde{U}^{\prime(t)}}\right)_{\mathrm{dis}},\left(\frac{\tilde{U}^{\prime(t)}}{\tilde{U}^{\prime(t)}}\right)_{\mathrm{dis}}\right\rangle .
$$

The trick here is to introduce a correction function

$$
s \mapsto \theta(s):=s^{2}\left\langle\left(\begin{array}{cc}
3 \phi_{0}^{4} & 2 \phi_{0}^{4} \\
2 \phi_{0}^{4} & 3 \phi_{0}^{4}
\end{array}\right)\left(\frac{\tilde{U}^{\prime(t)}}{\tilde{U}^{\prime(t)}}\right)_{\mathrm{dis}}(s, \cdot),\left(\frac{\tilde{U}^{\prime(t)}}{\tilde{U}^{\prime(t)}}\right)_{\mathrm{dis}}(s, \cdot)\right\rangle .
$$

If one applies the time derivative to $\theta(s)$, the main contribution comes from the terms when $\left(\frac{\tilde{U}^{\prime(t)}}{\tilde{U}^{\prime(t)}}\right)_{\text {dis }}$ gets hit. Otherwise, one obtains at least an extra $\partial_{s}\left[\Psi-\Psi_{\infty}\right]$, which makes the expression absolutely integrable. Thus $\theta^{\prime}(s)$ equals up to negligible errors 


$$
\begin{aligned}
i \theta^{\prime}(s) \sim & s^{2}\left(\begin{array}{ll}
3 \phi_{0}^{4} & 2 \phi_{0}^{4} \\
2 \phi_{0}^{4} & 3 \phi_{0}^{4}
\end{array}\right)\left\langle i \partial_{s}\left(\frac{\tilde{U}^{\prime(t)}}{\tilde{U}^{\prime(t)}}\right)_{\mathrm{dis}},\left(\frac{\tilde{U}^{\prime(t)}}{\tilde{U}^{\prime(t)}}\right)_{\mathrm{dis}}\right\rangle \\
& -s^{2}\left\langle\left(\begin{array}{ll}
3 \phi_{0}^{4} & 2 \phi_{0}^{4} \\
2 \phi_{0}^{4} & 3 \phi_{0}^{4}
\end{array}\right)\left(\frac{\tilde{U}^{\prime(t)}}{\tilde{U}^{\prime(t)}}\right)_{\mathrm{dis}}, i \partial_{s}\left(\frac{\tilde{U}^{\prime(t)}}{\tilde{U}^{\prime(t)}}\right)_{\mathrm{dis}}\right\rangle \\
= & s^{2}\left\langle\left(\begin{array}{ll}
3 \phi_{0}^{4} & 2 \phi_{0}^{4} \\
2 \phi_{0}^{4} & 3 \phi_{0}^{4}
\end{array}\right)\left[-\mathcal{H}_{0}\left(\frac{\tilde{U}^{\prime(t)}}{\tilde{U}^{\prime(t)}}\right)_{\mathrm{dis}}-\left(\begin{array}{cc}
3 \phi_{0}^{4} & 2 \phi_{0}^{4} \\
-2 \phi_{0}^{4} & -3 \phi_{0}^{4}
\end{array}\right)\left(\frac{\tilde{U}^{\prime(t)}}{\tilde{U}^{\prime(t)}}\right)_{\mathrm{dis}} \ldots\right],\left(\frac{\tilde{U}^{\prime(t)}}{\tilde{U}^{\prime(t)}}\right)_{\mathrm{dis}}\right\rangle \\
- & s^{2}\left\langle\left(\begin{array}{ll}
3 \phi_{0}^{4} & 2 \phi_{0}^{4} \\
2 \phi_{0}^{4} & 3 \phi_{0}^{4}
\end{array}\right)\left(\frac{\tilde{U}^{\prime(t)}}{\tilde{U}^{\prime(t)}}\right)_{\mathrm{dis}},-\mathcal{H}_{0}\left(\frac{\tilde{U}^{\prime(t)}}{\tilde{U}^{\prime(t)}}\right)_{\mathrm{dis}}-\left(\begin{array}{cc}
3 \phi_{0}^{4} & 2 \phi_{0}^{4} \\
-2 \phi_{0}^{4} & -3 \phi_{0}^{4}
\end{array}\right)\left(\frac{\tilde{U}^{\prime(t)}}{\tilde{U}^{\prime(t)}}\right)_{\mathrm{dis}}+\cdots\right\rangle .
\end{aligned}
$$

Here the terms ... are absolutely integrable in $s$ after spatial localization and multiplying with $s^{2}$. Now observe the matrix identity

$$
\left(\begin{array}{cc}
3 \phi_{0}^{4} & 2 \phi_{0}^{4} \\
2 \phi_{0}^{4} & 3 \phi_{0}^{4}
\end{array}\right)\left(\begin{array}{cc}
3 \phi_{0}^{4} & 2 \phi_{0}^{4} \\
-2 \phi_{0}^{4} & -3 \phi_{0}^{4}
\end{array}\right)=\left(\begin{array}{cc}
3 \phi_{0}^{4} & -2 \phi_{0}^{4} \\
2 \phi_{0}^{4} & -3 \phi_{0}^{4}
\end{array}\right)\left(\begin{array}{cc}
3 \phi_{0}^{4} & 2 \phi_{0}^{4} \\
2 \phi_{0}^{4} & 3 \phi_{0}^{4}
\end{array}\right) .
$$

Thus up to good error terms, we can reduce 5.16 to the commutator expression

$$
-\left\langle s^{2}\left[\left(\begin{array}{cc}
3 \phi_{0}^{4} & 2 \phi_{0}^{4} \\
2 \phi_{0}^{4} & 3 \phi_{0}^{4}
\end{array}\right), \mathcal{H}_{0}\right]\left(\frac{\tilde{U}^{\prime(t)}}{\tilde{U}^{\prime(t)}}\right)_{\mathrm{dis}},\left(\frac{\tilde{U}^{\prime(t)}}{\tilde{U}^{\prime(t)}}\right)_{\mathrm{dis}}\right\rangle .
$$

This last expression is of the form

$$
s^{2}\left\langle\left(\tilde{U}_{\mathrm{dis}}^{\prime(t)}\right)^{2}-\left(\overline{\tilde{U}_{\mathrm{dis}}^{\prime(t)}}\right)^{2}, \phi\right\rangle
$$

for a certain time independent Schwartz function $\phi$, and hence integrable according to the assumptions of the lemma. Thus to conclude the proof of the lemma, we need to control the remaining terms in 5.15). We demonstrate this for the expressions of the schematic form

$$
s^{2}\left\langle\dot{\pi} \partial_{\pi} W,\left(\begin{array}{c}
\tilde{\tilde{U}} \\
\tilde{\tilde{U}}
\end{array}\right)_{\mathrm{dis}}\right\rangle, \quad s^{2}\left\langle\dot{\pi} \partial_{\pi} W, \partial_{y}^{i}\left(\begin{array}{c}
\tilde{U} \\
\tilde{\tilde{U}}
\end{array}\right)_{\mathrm{dis}}\right\rangle, \quad i=1,2, \quad\left\langle C_{1}\left(\begin{array}{c}
\tilde{U} \\
\tilde{\tilde{U}}
\end{array}\right)_{\mathrm{dis}},\left(\begin{array}{c}
|\tilde{U}|^{4} \tilde{U} \\
-|\tilde{U}|^{4} \overline{\tilde{U}}
\end{array}\right)\right\rangle,
$$

the other terms implied in $N(\tilde{U}, \pi)$ being local and at least quadratic in $\tilde{U}$ and hence leading to straightforward contributions. Assuming control over the preceding terms, we then obtain

$$
\left|\int_{0}^{T} i \partial_{s}\left\langle C_{1}\left(\frac{\tilde{U}^{\prime(t)}}{\tilde{U}^{\prime(t)}}\right)_{\mathrm{dis}},\left(\frac{\tilde{U}^{\prime(t)}}{\tilde{U}^{\prime(t)}}\right)_{\mathrm{dis}}\right\rangle d s-\frac{\theta(s)}{4}\right|_{0}^{T} \mid \leq \frac{R^{2}}{100} \delta^{2} .
$$

This easily implies the assertion of the lemma by choosing $\delta$ small enough in relation to $R$. Thus to conclude, first consider $s^{2}\left\langle\dot{\pi} \partial_{\pi} W,\left(\frac{\tilde{U}^{\prime(t)}}{\tilde{U}^{\prime(t)}}\right)_{\text {dis }}(s, \cdot)\right\rangle$. Using $[3.6$, we see that these are all equivalent to expressions of the form

$$
s^{2}(v-1)(s)\left\langle\tilde{U}^{2}, \phi\right\rangle, \quad s^{2} \lambda_{6}^{2}(s), \quad s^{2} \lambda_{6}(s)\langle\tilde{U}, \phi\rangle
$$


as well as terms of higher order in $U, \lambda_{6}$. All of these are easily seen to be absolutely integrable in light of our decay assumptions.

We have to argue a bit differently for the expressions $s^{2}\left\langle\dot{\pi} \partial_{\pi} W, \partial_{y}^{i}\left(\frac{\tilde{U}^{\prime(t)}}{\tilde{U}^{(t)}}\right)_{\mathrm{dis}}\right\rangle, i=1,2$, as we have not built the strong local decay rate $s^{-3 / 2}$ into the estimates for the derivatives of the dispersive radiation part. In this case, we need to leak a little extra: for a Schwartz function $\phi$ write

$$
\phi \partial_{y}\left(\tilde{U}_{\mathrm{dis}}^{\prime(t)}(s, \cdot)\right)=\phi P_{<\epsilon \in(1 / N)} \partial_{y}\left(\tilde{U}_{\mathrm{dis}}^{\prime(t)}(s, \cdot)\right)+\phi P_{\geq s^{\epsilon(1 / N)}} \partial_{y}\left(\tilde{U}_{\mathrm{dis}}^{\prime(t)}(s, \cdot)\right) .
$$

Note that if we choose $\epsilon(1 / N)$ large enough, we can ensure that

$$
\left\|\phi P_{\geq s^{\epsilon(1 / N)}} \partial_{y}\left(\tilde{U}_{\text {dis }}^{\prime(t)}(s, \cdot)\right)\right\|_{L_{x}^{1}} \lesssim\langle s\rangle^{-N_{0}}
$$

for large $N_{0}=N_{0}(N)$. Next, use a compactly supported partition of unity $\left\{\phi_{j}\right\}$ with $\phi_{0}(y)$ centered at $y=0$ to write

$$
\phi P_{<s^{\epsilon(1 / N)}} \partial_{y}\left(\tilde{U}_{\mathrm{dis}}^{\prime(t)}(s, \cdot)\right)=\sum_{j} \phi P_{<s^{\epsilon(1 / N)}} \partial_{y}\left(\phi_{j} \tilde{U}_{\mathrm{dis}}^{\prime(t)}(s, \cdot)\right) . .
$$

Then we have

$$
\left\|\phi P_{<s \in(1 / N)} \partial_{y}\left(\phi_{j} \tilde{U}_{\text {dis }}^{\prime(t)}(s, \cdot)\right)\right\|_{L_{x}^{1}} \lesssim j^{-\tilde{N}}
$$

for any $\tilde{N}$ and $j>s^{\epsilon_{1}(1 / N)}$, whence we may restrict to $j \leq s^{\epsilon_{1}(1 / N)}$. In that case, use the fact that the proof of the preceding lemma improving the strong local decay only required control of finitely many weighted estimates involving $\phi$ and its derivatives to conclude that

$$
\left\|\phi P_{<s^{\epsilon(1 / N)}} \partial_{y}\left(\phi_{j} \tilde{U}_{\mathrm{dis}}^{\prime(t)}(s, \cdot)\right)\right\|_{L_{x}^{1}} \lesssim s^{\epsilon_{2}(1 / N)} s^{-3 / 2} .
$$

Thus one obtains in summary (with a similar estimate for the second derivative)

$$
\left\|\phi \partial_{y} \tilde{U}_{\text {dis }}^{\prime(t)}(s, \cdot)\right\|_{L_{x}^{1}} \lesssim\langle s\rangle^{-3 / 2+\epsilon(1 / N)} .
$$

Then one can proceed as before to estimate $s^{2}\left\langle\dot{\pi} \partial_{\pi} W, \partial_{y}^{1,2} \tilde{U}_{\text {dis }}^{\prime(t)}\right\rangle$. Finally, we turn to the non-local expression

$$
\begin{aligned}
& \left\langle C\left(\begin{array}{c}
|\tilde{U}|^{4} \tilde{U} \\
-|\tilde{U}|^{4} \tilde{U}
\end{array}\right)(t, \cdot),\left(\frac{\tilde{U}^{\prime(t)}}{\tilde{U}^{\prime(t)}}\right)_{\mathrm{dis}}(t, \cdot)\right\rangle \\
& \quad=\left\langle\left(\begin{array}{cc}
y-2 s p & 0 \\
0 & y+2 s p
\end{array}\right)\left(\begin{array}{c}
|\tilde{U}|^{4} \tilde{U} \\
-|\tilde{U}|^{4} \overline{\tilde{U}}
\end{array}\right)(t, \cdot),\left(\begin{array}{cc}
y-2 s p & 0 \\
0 & y+2 s p
\end{array}\right)\left(\frac{\tilde{U}^{\prime(t)}}{\tilde{U}^{\prime(t)}}\right)_{\mathrm{dis}}(t, \cdot)\right\rangle .
\end{aligned}
$$

Decompose $\tilde{U}$ into its dispersive and root part. The latter is easily seen to lead to a controllable contribution, so we now replace $\tilde{U}$ by $\tilde{U}_{\text {dis }}$. Then use the identity

$$
(y-2 s p)\left[\left|\tilde{U}_{\mathrm{dis}}\right|^{4} \tilde{U}_{\mathrm{dis}}\right]=-2 s p\left[\left|\tilde{U}_{\mathrm{dis}}\right|^{4}\right] \tilde{U}_{\mathrm{dis}}+\left|\tilde{U}_{\mathrm{dis}}\right|^{4}(y-2 s p) \tilde{U}_{\mathrm{dis}} .
$$

Also, we have

$2 s p\left[\left|\tilde{U}_{\mathrm{dis}}\right|^{4}\right]=4 s p\left[\left|\tilde{U}_{\mathrm{dis}}\right|^{2}\right]\left|\tilde{U}_{\mathrm{dis}}\right|^{2}=2\left[-(y-2 s p) \tilde{U}_{\mathrm{dis}} \overline{\tilde{U}}_{\mathrm{dis}}+\tilde{U}_{\mathrm{dis}} \overline{(y-2 s p) \tilde{U}_{\mathrm{dis}}}\right]\left|\tilde{U}_{\mathrm{dis}}\right|^{2}$. 
Thus we can expand the integrand in 5.17) as a sum of expressions of the form

$$
\left[(y-2 s p) \tilde{U}_{\mathrm{dis}}^{\prime}\right]\left[(y+2 s p) \overline{\tilde{U}}_{\mathrm{dis}}\right]\left|\tilde{U}_{\mathrm{dis}}\right|^{4}
$$

plus similar terms. We can bound its $L_{y}^{1}$-norm by $\lesssim R^{6} \delta^{6}\langle s\rangle^{-2+\epsilon\left(\delta_{2}\right)}$ and hence integrable with respect to $s$.

For the second statement of the lemma, one decomposes

$$
C \partial_{y} \tilde{U}^{\prime(t)}=P_{>s^{\epsilon(1 / N)}} C \partial_{y} \tilde{U}^{\prime(t)}+P_{\leq s^{\epsilon(1 / N)}} C \partial_{y} \tilde{U}^{\prime(t)} .
$$

For the first part, one reiterates the preceding proof, but uses the much improved decay estimates due to the frequency localization and our assumptions on higher derivatives. For the low frequency part, one leaks $\langle s\rangle^{\epsilon(1 / N)}$, which is of course much better than $\langle s\rangle^{1 / 2}$ upon choosing $N$ large enough. This concludes the proof of Lemma 5.8

In order to complete our control of the radiation part, we now need to improve the estimates for the root part of the radiation. We shall take decisive advantage here of the iterated structure of the spaces $A_{0, T)}^{(n)}$.

Lemma 5.9. Let $\Gamma_{T}^{\prime}=T_{A, \lambda, \beta, \omega, \gamma, \mu} \Gamma_{T}$ be an element of $A_{[0, T)}^{(10)}$. Then

$$
\sup _{0 \leq t \leq T} \sup _{0 \leq k \leq[N / 3]}\left\|\langle T\rangle^{2-4 \delta_{1}} \frac{d^{k}}{d t^{k}} \lambda_{i}^{\prime}(t)\right\|_{L_{t}^{M}} \leq \frac{R}{100} \delta^{2} .
$$

Proof. Recalling 3.18, 3.17), we see that it suffices to prove the claim (if necessary with improved right hand side) for $\lambda_{6}(t)$. To do so we recall the relation $(3.23)$ as well as (3.24). Expanding the phase $e^{\Lambda(s)}$ in a Taylor expansion, we obtain a number of terms, of which the following have the least temporal decay: $(v-1)^{a}\left\langle\left(\begin{array}{c}\tilde{U} \\ \tilde{U}\end{array}\right)_{\mathrm{dis}}, \phi\right\rangle, a=1,2$. Indeed, all the others are easily seen to have decay rate at least $\langle s\rangle^{-3+2 \delta_{1}}$. Now use the fact that $\left(\begin{array}{c}\tilde{U} \\ \tilde{U}\end{array}\right)_{\text {dis }}$ is a convex linear combination of functions $\left(\begin{array}{c}\tilde{U}_{i} \\ \tilde{U}_{i}\end{array}\right)_{\text {dis }}$, each of which is obtained as in 3.27). It clearly suffices to consider the contribution of a single such function; we shall omit the subscript for simplicity. Using the Duhamel formula (3.27) and recalling the precise structure of its right hand side, and plugging everything into 3.24 , we then need to estimate, amongst others, the expressions 52 (in the case $a=1$, the case $a=2$ being similar)

$$
\begin{aligned}
\int_{t}^{\infty}(v-1)(s) & \left\langle\int_{0}^{s} e^{i(s-\lambda) \mathcal{H}}\right. \\
\cdot\left[\left(e^{2 i\left(\Psi-\Psi_{\infty}\right)_{1}(s)-2 i\left(\Psi-\Psi_{\infty}\right)_{1}(\lambda)}-1\right.\right. & \left.-e^{-2 i\left(\Psi-\Psi_{\infty}\right)_{1}(s)+2 i\left(\Psi-\Psi_{\infty}\right)_{1}(\lambda)}+1\right) \\
& \left.\left.\times\left(\frac{\tilde{U}^{(s)}(\lambda, \cdot)}{\tilde{U}^{(s)}(\lambda, \cdot)}\right) \phi\right]_{\mathrm{dis}}, \phi\right\rangle d \lambda d s,
\end{aligned}
$$

\footnotetext{
52 Recall also the proof of Lemma 5.4
} 


$$
\int_{t}^{\infty}(v-1)(s)\left\langle\int_{0}^{s} e^{i(s-\lambda) \mathcal{H}}\left(\begin{array}{c}
\left|\tilde{U}^{(s)}\right|^{4} \tilde{U}^{(s)}(\lambda, \cdot) \\
-\left|\tilde{U}^{(s)}\right|^{4} \tilde{U}^{(s)}(\lambda, \cdot)
\end{array}\right)_{\mathrm{dis}}, \phi\right\rangle d \lambda d s
$$

Of course we are using schematic notation here, the symbol $\phi$ denoting various Schwartz functions, which also may depend on time, and are more precisely of the form $C(\Psi-$ $\left.\Psi_{\infty}, v, \lambda_{\infty}\left(\mu-\mu_{\infty}\right)\right)$ for some smooth function $C(\cdot, \cdot, \cdot)$ satisfying uniform estimates with respect to its three arguments. Also, we commit abuse of notation and do not differentiate between the inputs used for the Duhamel source term and the outputs. We commence by estimating the first of these terms: introduce the matrix-valued function

$$
\phi(s, \lambda, y):=\left(\begin{array}{cc}
0 & -e^{-2 i\left(\Psi-\Psi_{\infty}\right)_{1}(s)+2 i\left(\Psi-\Psi_{\infty}\right)_{1}(\lambda)}+1 \\
e^{2 i\left(\Psi-\Psi_{\infty}\right)_{1}(s)-2 i\left(\Psi-\Psi_{\infty}\right)_{1}(\lambda)}-1 & 0
\end{array}\right) \phi
$$

Using the Plancherel's Theorem 2.3 for the distorted Fourier transform, we can then express the first term above as

$\sum_{ \pm} \int_{-\infty}^{\infty} \int_{t}^{\infty}(v-1)(s) \int_{0}^{s} e^{ \pm i(s-\lambda)\left(\xi^{2}+1\right)} \mathcal{F}_{ \pm}\left[\phi(s, \lambda, x)\left(\frac{\tilde{U}^{(s)}}{\tilde{U}^{(s)}}\right)(\lambda, \cdot)\right](\xi) \overline{\tilde{\mathcal{F}}_{ \pm} \phi(\xi)} d \lambda d s d \xi$

We perform an integration by parts in the $s$-variable, replacing the above by the following terms:

$$
\begin{aligned}
& \sum_{ \pm} \int_{-\infty}^{\infty}(v-1)(t) \int_{0}^{t} e^{ \pm i(t-\lambda)\left(\xi^{2}+1\right)} \mathcal{F}_{ \pm}\left[\phi(t, \lambda, x)\left(\frac{\tilde{U}^{(t)}}{\tilde{U}^{(t)}}\right)(\lambda)\right](\xi) \frac{\overline{\tilde{\mathcal{F}}_{ \pm} \phi(\xi)}}{\xi^{2}+1} d \lambda d \xi \\
& \sum_{ \pm} \int_{-\infty}^{\infty} \int_{t}^{\infty}(v-1)(s) \int_{0}^{s} e^{ \pm i(s-\lambda)\left(\xi^{2}+1\right)} \mathcal{F}_{ \pm}\left[\partial_{s} \phi(s, \lambda, x)\left(\frac{\tilde{U}^{(s)}}{\tilde{U}^{(s)}}\right)\right](\xi) \frac{\overline{\tilde{\mathcal{F}}_{ \pm} \phi(\xi)}}{\xi^{2}+1} d \lambda d s d \xi
\end{aligned}
$$

$\sum_{ \pm} \int_{-\infty}^{\infty} \int_{t}^{\infty}(v-1)(s) \partial_{s}\left[\lambda_{\infty}\left(\mu-\mu_{\infty}\right)\right](s)$

$$
\times \int_{0}^{s} e^{ \pm i(s-\lambda)\left(\xi^{2}+1\right)} \mathcal{F}_{ \pm}\left[\phi(s, \lambda, x) \partial_{y}\left(\frac{\tilde{U}^{(s)}}{\tilde{U}^{(s)}}\right)\right](\xi) \frac{\overline{\tilde{\mathcal{F}}_{ \pm} \phi(\xi)}}{\xi^{2}+1} d \lambda d s d \xi
$$

$\sum_{ \pm} \int_{-\infty}^{\infty} \int_{t}^{\infty} \dot{v}(s) \int_{0}^{s} e^{ \pm i(s-\lambda)\left(\xi^{2}+1\right)} \mathcal{F}_{ \pm}\left[\phi(s, \lambda, x)\left(\frac{\tilde{U}^{(s)}}{\tilde{U}^{(s)}}\right)(\lambda, \cdot)\right](\xi) \frac{\overline{\tilde{\mathcal{F}}_{ \pm} \phi(\xi)}}{\xi^{2}+1} d \lambda d s d \xi$

Most of these are almost immediate to estimate. Note that upon undoing the Fourier transform in the first expression, we obtain

$$
(v-1)(t) \int_{0}^{t}\left\langle e^{i(t-\lambda) \mathcal{H}}\left[\left(\begin{array}{c}
\tilde{U}(\lambda, \cdot) \\
\tilde{\tilde{U}}(\lambda, \cdot)
\end{array}\right) \phi(t, \lambda, x)\right]_{\mathrm{dis}},\left(\mathcal{H}^{*}\right)^{-1} \phi(x)\right\rangle d \lambda
$$


We claim that $\left\|x^{2} \mathcal{H}^{-1} \phi\right\|_{L_{x}^{1+}} \lesssim O(1)$, where $1+$ can be chosen arbitrarily close to 1 . Write

$$
\mathcal{H}^{-1} \phi=\sum_{ \pm} \int_{-\infty}^{\infty} e_{ \pm}(x, \xi) \frac{\tilde{\mathcal{F}}_{ \pm}(\phi)(\xi)}{\xi^{2}+1} d \xi
$$

We treat here the + part, the other one being similar. We equate the preceding for $x>0$ with

$$
\begin{aligned}
\int_{0}^{\infty}\left[s(\xi) e^{i x \xi} \underline{e}+\phi(x, \xi)\right] & \frac{\mathcal{F}_{ \pm}(\phi)(\xi)}{\xi^{2}+1} d \xi \\
& +\int_{-\infty}^{0}\left(\left[e^{i x \xi}+r(-\xi) e^{-i x \xi}\right] \underline{e}+\phi(x, \xi)\right) \frac{\mathcal{F}(\phi)(\xi)}{\xi^{2}+1} d \xi .
\end{aligned}
$$

For the first integral, omitting the exponentially decaying local part, we have

$$
\begin{aligned}
\int_{0}^{\infty} s(\xi) e^{i x \xi} & \underline{e} \frac{\mathcal{F}_{ \pm}(\phi)(\xi)}{\xi^{2}+1} d \xi \\
= & -\frac{1}{i x} \int_{0}^{\infty}\left(\partial_{\xi}[s(\xi)] e^{i x \xi} \frac{\mathcal{F}_{ \pm}(\phi)(\xi)}{\xi^{2}+1} \underline{e}+s(\xi) e^{i x \xi} \underline{e} \partial_{\xi}\left[\frac{\mathcal{F}_{ \pm}(\phi)(\xi)}{\xi^{2}+1}\right]\right) d \xi \\
= & \frac{1}{\left(-x^{2}\right)} \int_{0}^{\infty} e^{i x \xi} \partial_{\xi}\left(\partial_{\xi}[s(\xi)] \frac{\mathcal{F}_{ \pm}(\phi)(\xi)}{\xi^{2}+1} \underline{e}\right) d \xi \\
& +\frac{1}{\left(-x^{2}\right)} \int_{0}^{\infty} e^{i x \xi} \partial_{\xi}\left[s(\xi) \underline{e} \partial_{\xi}\left[\frac{\mathcal{F}_{ \pm}(\phi)(\xi)}{\xi^{2}+1}\right]\right] d \xi=O\left(\frac{1}{x^{3}}\right) .
\end{aligned}
$$

Similarly (omitting the contributions from $1+r(-\xi), \phi(x, \xi)$ ), we have

$$
\begin{aligned}
\int_{-\infty}^{0} \frac{e^{i x \xi}-e^{-i x \xi}}{\xi^{2}+1} \underline{e} \mathcal{F}_{ \pm}(\phi)(\xi) d \xi=-\frac{1}{i x} \int_{-\infty}^{0}\left[e^{i x \xi}+e^{-i x \xi}\right] \underline{e} \partial_{\xi}\left[\frac{\mathcal{F}_{ \pm} \phi(\xi)}{\xi^{2}+1}\right] d \xi \\
=\frac{1}{\left(-x^{2}\right)} \int_{-\infty}^{0}\left(e^{i x \xi}-e^{-i x \xi}\right) \underline{e} \partial_{\xi}^{2}\left[\frac{\mathcal{F}_{ \pm} \phi(\xi)}{\xi^{2}+1}\right] d \xi=O\left(\frac{1}{x^{3}}\right) .
\end{aligned}
$$

If we then repeat the steps in the proof of the strong local dispersive estimate, we get

$$
\begin{aligned}
& \left|(\nu-1)(t) \int_{0}^{t}\left\langle e^{ \pm i(t-\lambda) \mathcal{H}}\left[\left(\frac{\tilde{U}(\lambda, \cdot)}{\tilde{U}(\lambda, \cdot)}\right) \phi(t, \lambda, x)\right]_{\mathrm{dis}},\left(\mathcal{H}^{*}\right)^{-1} \phi(x)\right\rangle d \lambda\right| \\
& \lesssim t^{-1 / 2+\delta_{1}} t^{-3 / 2+2 \delta_{3}},
\end{aligned}
$$

as desired. In the expression 5.21, note that we have the schematic identity

$$
\begin{aligned}
\partial_{s} \phi(s, \lambda, y)= & \partial_{s}\left[\Psi-\Psi_{\infty}\right] \partial_{1} C\left(\Psi-\Psi_{\infty}, v, \lambda_{\infty}\left(\mu-\mu_{\infty}\right)\right) \\
& +\frac{d}{d s}[\nu] \partial_{2} C\left(\Psi-\Psi_{\infty}, \nu, \lambda_{\infty}\left(\mu-\mu_{\infty}\right)\right) \\
& +\frac{d}{d s}\left[\lambda_{\infty}\left(\mu-\mu_{\infty}\right)\right] \partial_{3} C\left(\Psi-\Psi_{\infty}, \nu, \lambda_{\infty}\left(\mu-\mu_{\infty}\right)\right) .
\end{aligned}
$$


We have $\partial_{s}\left[\Psi-\Psi_{\infty}\right]=v-1+$ error. Now perform at most two additional integrations by parts with respect to $s$ in 5.21. This either produces additional factors of at least the decay $v-1$, or else kills the integral over $\lambda$, in which case one arrives at an expression 53

$$
\int_{t}^{\infty}(v-1)(s) \frac{d}{d s}\left[\Psi-\Psi_{\infty}\right]_{1}(s)\left\langle\left(\begin{array}{c}
\overline{\tilde{U}} \\
\tilde{U}
\end{array}\right)(s, \cdot) \phi(x),\left(\mathcal{H}^{*}\right)^{-2} \phi_{\mathrm{dis}}\right\rangle d s .
$$

In the former case, one can close directly, while in the latter case, use the customary decomposition of $\left(\begin{array}{c}\tilde{U} \\ \tilde{U}\end{array}\right)$ into dispersive and root part. Recycling (3.18), we thus see that up to an integral of the form

$$
\int_{t}^{\infty}(v-1)^{2}(t) \lambda_{6}(t) d t
$$

we arrive at the expression we started out with but with an extra weight of at least the strength $(v-1)(s) \frac{d}{d s}\left[\Psi-\Psi_{\infty}\right]_{1}(s) \sim(v(s)-1)^{2}$. Now iterate the procedure. The integral 5.25) can be estimated using Proposition 5.10, which will be proved independently below. The remaining terms after (5.21) are simpler; indeed, they can be integrated absolutely to give the desired upper bound.

Now consider 5.19). Here we also pass to the Fourier side, perform an integration by parts in $s$, and undo the Fourier transform. This results in the extra weights $\partial_{s}\left[\Psi-\Psi_{\infty}\right]_{1}$, $\partial_{s}\left[\lambda_{\infty}\left(\mu-\mu_{\infty}\right)\right]$, or else one winds up with the expression

$$
\int_{t}^{\infty}\left\langle\left(\begin{array}{c}
|\tilde{U}|^{4} \tilde{U}(s) \\
-|\tilde{U}|^{4} \tilde{U}(s)
\end{array}\right)_{\mathrm{dis}}, \mathcal{H}^{-1} \phi\right\rangle d s
$$

The former cases are treated by repeating integration by parts in $s$ if necessary and proceeding as in the proof of the strong local dispersive estimate (Lemma 5.7) with $\phi$ replaced by $\mathcal{H}^{-1} \phi$, while in the latter case we simply estimate (using the bound derived above on $\left.\left\|\langle x\rangle^{2} \mathcal{H}^{-1} \phi\right\|_{L_{x}^{1+}}\right)$

$$
\left|\int_{t}^{\infty}\left\langle\left(\begin{array}{c}
|\tilde{U}|^{4} \tilde{U}(s) \\
-|\tilde{U}|^{4} \tilde{U}(s)
\end{array}\right)_{\mathrm{dis}}, \mathcal{H}^{-1} \phi\right\rangle d s\right| \lesssim \int_{t}^{\infty} s^{-9 /\left(2+\epsilon\left(\delta_{2}\right)\right)} d s \lesssim t^{-2+2 \delta_{1}}
$$

Finally, obtaining estimates for $\frac{d^{k}}{d t^{k}} \lambda_{6}, 1 \leq k \leq[N / 3]$, is straightforward upon differentiating (3.23). This completes the proof of the lemma, up to handling (5.25), which is done using Proposition 5.10 below.

Note that the preceding six lemmas give us control over the radiation part. Now we need to obtain control over the modulation parameters. This will be more difficult and in particular build sensitively on the foundations provided in the preceding subsection. We first need to establish some bilinear estimates.

53 Using the distorted Plancherel's Theorem 2.3 


\subsection{Second stage. Estimates for certain quadratic expressions involving the radiation} part

We now need to refine our estimates for the radiation part. Specifically, inspection of 3.7 etc. reveals that certain expressions quadratic in $\tilde{U}_{\text {dis }}(s, \cdot)$ need to be integrated against suitable weights of $s$. Indeed, the most delicate of these expressions have borderline decay in light of the strong local dispersive decay. The only way to estimate these expressions, then, is to exploit some additional oscillatory behavior.

Proposition 5.10. Let $\Gamma_{T}^{\prime} \in A^{(10)}[0, T)$. Then writing $\Gamma_{T}^{\prime}=T_{A, \lambda, \beta, \omega, \gamma, \mu} \Gamma_{T}$, we have

$$
\left|\int_{t}^{T} s \lambda_{6}^{\prime}(s) d s\right| \lesssim \delta^{2}\langle T\rangle^{-1 / 2+\delta_{1}}
$$

Also,

$$
\left|\int_{t}^{T}(v-1)^{a} \lambda_{6}^{\prime}(s) d s\right| \lesssim \delta^{2}\langle T\rangle^{-3 / 2+\delta_{1}+a\left(-1 / 2+\delta_{1}\right)} .
$$

Proof. The proof shall hinge on two auxiliary lemmas, which pivotally improve on the work in the preceding subsection. We refer to (3.23), which we solve via (3.24). Expanding the phase $e^{\Lambda(s)}$, we need to estimate a list of terms. We shall use the definition of $A^{(10)}[0, T)$ to expand $\dot{v}$ as a convex linear combination of terms each of which is defined as the right hand side of (3.7) with respect to a tuple $\Gamma_{T} \in A^{(9)}[0, T)$. It suffices to consider the contribution of one such term in the convex linear combination. We now consider the contributions of a representative list of terms arising from 3.23:

(A) The contribution of the term $\int_{T}^{\infty} t \int_{t}^{\infty}(v-1)(s)\left\langle\left(\begin{array}{c}\tilde{U} \\ \tilde{U}\end{array}\right)_{\text {dis }}, \phi\right\rangle d s d t$. Recalling the discussion preceding (3.23), the Schwartz function $\phi$ here is itself dependent on time $s$, and more precisely, is of the form $C\left(v-1,\left(\Psi-\Psi_{\infty}\right)_{2}, \lambda_{\infty}\left(\mu-\mu_{\infty}\right)\right)$. Then expand $C(\cdot, \cdot, \cdot)$ into a finite Taylor expansion, where the cutoff is chosen far enough such that the error automatically leads to an acceptable contribution. Then, up to terms treatable by the exact same procedure, we see that we may assume that $\phi$ is a time independent Schwartz function. Now, write $\left(\begin{array}{c}\tilde{U} \\ \tilde{U}\end{array}\right)_{\text {dis }}$ as a convex linear combination of functions $\left(\begin{array}{c}\tilde{U} \\ \tilde{U}\end{array}\right)_{i \text {, dis }}$, each of which is given by the right hand side of 3.27) with respect to suitable tuples $\Gamma_{i, T}$. Again it suffices to consider the contribution of a single such term $(\underset{\tilde{U}}{\tilde{U}})_{i \text {,dis }}$. As we shall always use the same estimates, we will no longer differentiate between different tuples here. Now, plugging in the Duhamel expansion for $\left(\frac{\tilde{U}}{\tilde{U}}\right)_{i}$, dis , and abusing notation by omitting subscripts, and recalling the structure of the terms for example from the proof of Lemma 5.4. we see that we need to estimate the following schematically written expressions"

$$
\begin{aligned}
& \int_{T}^{\infty} t \int_{t}^{\infty}(v-1)(s) \\
& \cdot\left\langle\int_{0}^{s} e^{i(s-\lambda) \mathcal{H}}\left[\left(\begin{array}{c}
\left(1-e^{2 i\left(\Psi-\Psi_{\infty}\right)_{1}(\lambda)-2 i\left(\Psi-\Psi_{\infty}\right)_{1}(s)} \overline{\tilde{U}^{(s)}}(\lambda)\right. \\
\left(-1+e^{-2 i\left(\Psi-\Psi_{\infty}\right)_{1}(\lambda)+2 i\left(\Psi-\Psi_{\infty}\right)_{1}(s)}\right) \tilde{U}^{(s)}(\lambda)
\end{array}\right) \phi\right]_{\mathrm{dis}} d \lambda, \phi\right\rangle d s d t \\
& \int_{T}^{\infty} t \int_{t}^{\infty}(v-1)(s)\left(\int_{0}^{s} e^{i(s-\lambda) \mathcal{H}}\left(\begin{array}{c}
\left|\tilde{U}^{(s)}\right|^{4} \tilde{U}^{(s)}(\lambda, \cdot) \\
-\left|\tilde{U}^{(s)}\right|^{4} \tilde{\tilde{U}}^{(s)}(\lambda, \cdot)
\end{array}\right)_{\mathrm{dis}} d \lambda, \phi\right\rangle d s d t
\end{aligned}
$$


as well as local terms with better decay behavior than the first expression; these can be treated analogously. Start with the first term. Our plan is to exploit the oscillatory behavior of the integrand: We perform an integration by parts in $s$, and replace it by the following list of terms upon going to the Fourier side. We leave out \pm for simplicity:

$$
\begin{aligned}
& \int_{-\infty}^{\infty} \int_{T}^{\infty} t(v-1)(t) \int_{0}^{t} e^{i(t-\lambda)\left(\xi^{2}+1\right)} \\
& \cdot \mathcal{F}\left[\left(\begin{array}{c}
\left(1-e^{2 i\left(\Psi-\Psi_{\infty}\right)_{1}(\lambda)-2 i\left(\Psi-\Psi_{\infty}\right)_{1}(t)}\right) \overline{\tilde{U}^{(t)}}(\lambda, \cdot) \\
\left(-1+e^{-2 i\left(\Psi-\Psi_{\infty}\right)_{1}(\lambda)+2 i\left(\Psi-\Psi_{\infty}\right)_{1}(t)}\right) \tilde{U}^{(t)}(\lambda, \cdot)
\end{array}\right) \phi\right](\xi) \frac{\overline{\mathcal{F}} \phi}{\xi^{2}+1} d \lambda d t d \xi, \\
& \int_{-\infty}^{\infty} \int_{T}^{\infty} t \int_{t}^{\infty} \dot{v}(s) \int_{0}^{s} e^{i(s-\lambda)\left(\xi^{2}+1\right)} \\
& \cdot \mathcal{F}\left[\left(\begin{array}{c}
\left(1-e^{2 i\left(\Psi-\Psi_{\infty}\right)_{1}(\lambda)-2 i\left(\Psi-\Psi_{\infty}\right)_{1}(s)}\right) \overline{\tilde{U}^{(s)}}(\lambda, \cdot) \\
\left(-1+e^{-2 i\left(\Psi-\Psi_{\infty}\right)_{1}(\lambda)+2 i\left(\Psi-\Psi_{\infty}\right)_{1}(s)}\right) \tilde{U}^{(s)}(\lambda, \cdot)
\end{array}\right) \phi\right](\xi) \frac{\overline{\mathcal{F}} \phi}{\xi^{2}+1} d \lambda d s d t d \xi, \\
& \int_{-\infty}^{\infty} \int_{T}^{\infty} t \int_{t}^{\infty}(v-1)(s) \frac{d}{d s}\left[\Psi-\Psi_{\infty}\right]_{1} \int_{0}^{s} e^{i(s-\lambda)\left(\xi^{2}+1\right)} \\
& \cdot \mathcal{F}\left[\left(\begin{array}{c}
e^{2 i\left(\Psi-\Psi_{\infty}\right)_{1}(\lambda)-2 i\left(\Psi-\Psi_{\infty}\right)_{1}(s)} \overline{\tilde{U}^{(s)}}(\lambda, \cdot) \\
e^{-2 i\left(\Psi-\Psi_{\infty}\right)_{1}(\lambda)+2 i\left(\Psi-\Psi_{\infty}\right)_{1}(s)} \tilde{U}^{(s)}(\lambda, \cdot)
\end{array}\right) \phi\right](\xi) \frac{\overline{\mathcal{F} \phi}}{\xi^{2}+1} d \lambda d s d t d \xi, \\
& \int_{-\infty}^{\infty} \int_{T}^{\infty} t \int_{t}^{\infty}(v-1)(s) \frac{d}{d s}\left[\lambda_{\infty}\left(\mu-\mu_{\infty}\right)\right] \int_{0}^{s} e^{i(s-\lambda)\left(\xi^{2}+1\right)} \\
& \cdot \mathcal{F}\left[\left(\begin{array}{c}
\left(1-e^{2 i\left(\Psi-\Psi_{\infty}\right)_{1}(\lambda)-2 i\left(\Psi-\Psi_{\infty}\right)_{1}(s)}\right) \overline{\partial_{x} \tilde{U}^{(s)}}(\lambda, \cdot) \\
\left(-1+e^{-2 i\left(\Psi-\Psi_{\infty}\right)_{1}(\lambda)+2 i\left(\Psi-\Psi_{\infty}\right)_{1}(s)}\right) \partial_{x} \tilde{U}^{(s)}(\lambda, \cdot)
\end{array}\right) \phi\right](\xi) \frac{\overline{\mathcal{F} \phi}}{\xi^{2}+1} d \lambda d s d t d \xi .
\end{aligned}
$$

A similar list of terms results from (5.26). We commence with the first term in this list. Perform an integration by parts in $t$, thereby replacing it by

$$
\begin{aligned}
& \int_{-\infty}^{\infty} T(v-1)(T) \int_{0}^{T} e^{i(T-\lambda)\left(\xi^{2}+1\right)} \\
& \cdot \mathcal{F}\left[\left(\begin{array}{c}
\left(1-e^{2 i\left(\Psi-\Psi_{\infty}\right)_{1}(\lambda)-2 i\left(\Psi-\Psi_{\infty}\right)_{1}(T)}\right) \overline{\tilde{U}^{(T)}}(\lambda, \cdot) \\
\left(-1+e^{2 i\left(\Psi-\Psi_{\infty}\right)_{1}(\lambda)-2 i\left(\Psi-\Psi_{\infty}\right)_{1}(T)}\right) \tilde{U}^{(T)}(\lambda, \cdot)
\end{array}\right) \phi\right](\xi) \frac{\overline{\mathcal{F}} \phi}{\left(\xi^{2}+1\right)^{2}} d \lambda d \xi, \\
& \int_{-\infty}^{\infty} \int_{T}^{\infty}(v-1)(t) \int_{0}^{t} e^{i(t-\lambda)\left(\xi^{2}+1\right)} \\
& \cdot \mathcal{F}\left[\left(\begin{array}{c}
\left(1-e^{2 i\left(\Psi-\Psi_{\infty}\right)_{1}(\lambda)-2 i\left(\Psi-\Psi_{\infty}\right)_{1}(t)}\right) \overline{\tilde{U}^{(t)}}(\lambda, \cdot) \\
\left(-1+e^{2 i\left(\Psi-\Psi_{\infty}\right)_{1}(\lambda)-2 i\left(\Psi-\Psi_{\infty}\right)_{1}(t)}\right) \tilde{U}^{(t)}(\lambda, \cdot)
\end{array}\right) \phi\right](\xi) \frac{\overline{\mathcal{F}} \phi}{\left(\xi^{2}+1\right)^{2}} d \lambda d t d \xi, \\
& \int_{-\infty}^{\infty} \int_{T}^{\infty} t(v-1)(t) \frac{d}{d t}\left[\Psi-\Psi_{\infty}\right]_{1}(t) \int_{0}^{t} e^{i(t-\lambda)\left(\xi^{2}+1\right)} \\
& \cdot \mathcal{F}\left[\left(\begin{array}{c}
\left(1+e^{2 i\left(\Psi-\Psi_{\infty}\right)_{1}(\lambda)-2 i\left(\Psi-\Psi_{\infty}\right)_{1}(t)}\right) \overline{\tilde{U}^{(t)}}(\lambda, \cdot) \\
\left(1+e^{-2 i\left(\Psi-\Psi_{\infty}\right)(\lambda)+2 i\left(\Psi-\Psi_{\infty}\right)(t)}\right) \tilde{U}^{(t)}(\lambda, \cdot)
\end{array}\right) \phi\right](\xi) \frac{\tilde{\mathcal{F}} \phi}{\left(\xi^{2}+1\right)^{2}} d \lambda d t d \xi
\end{aligned}
$$




$$
\begin{aligned}
& \int_{-\infty}^{\infty} \int_{T}^{\infty} t(v-1)(t) \frac{d}{d t}\left[\lambda_{\infty}\left(\mu-\mu_{\infty}\right)\right](t) \int_{0}^{t} e^{i(t-\lambda)\left(\xi^{2}+1\right)} \\
& \cdot \mathcal{F}\left[\left(\begin{array}{c}
\left(1-e^{2 i\left(\Psi-\Psi_{\infty}\right)_{1}(\lambda)-2 i\left(\Psi-\Psi_{\infty}\right)_{1}(t)} \overline{\partial_{x} \tilde{U}^{(t)}}(\lambda, \cdot)\right. \\
\left(-1+e^{-2 i\left(\Psi-\Psi_{\infty}\right)_{1}(\lambda)+2 i\left(\Psi-\Psi_{\infty}\right)_{1}(t)}\right) \partial_{x} \tilde{U}^{(t)}(\lambda, \cdot)
\end{array}\right) \phi\right](\xi) \frac{\overline{\mathcal{F}} \phi}{\left(\xi^{2}+1\right)^{2}} d \lambda d t d \xi .
\end{aligned}
$$

Each of these terms is straightforward to estimate: undo the Fourier transform, using the distorted Plancherel's Theorem 2.3, thereby replacing $\phi$ by $\mathcal{H}^{-2} \phi$, which again satisfies $\left\|x^{2} \mathcal{H}^{-2} \phi\right\|_{L_{x}^{1+}} \lesssim O(1)$. Thus, for example we get

$$
\begin{aligned}
& \mid \int_{-\infty}^{\infty} T(v-1)(T) \int_{0}^{T} e^{i(T-\lambda)\left(\xi^{2}+1\right)} \\
& \cdot \mathcal{F}\left[\left(\begin{array}{c}
\left(1-e^{2 i\left(\Psi-\Psi_{\infty}\right)_{1}(\lambda)-2 i\left(\Psi-\Psi_{\infty}\right)_{1}(T)} \overline{\tilde{U}^{(T)}}(\lambda)\right. \\
\left(-1+e^{-2 i\left(\Psi-\Psi_{\infty}\right)_{1}(\lambda)+2 i\left(\Psi-\Psi_{\infty}\right)_{1}(T)}\right) \tilde{U}^{(T)}(\lambda)
\end{array}\right) \phi\right](\xi) \frac{\overline{\mathcal{F}} \phi}{\left(\xi^{2}+1\right)^{2}} d \lambda d \xi \\
& \lesssim T^{1 / 2+\delta_{1}}\langle T\rangle^{-3 / 2} d T \lesssim T^{-1+\delta_{1}},
\end{aligned}
$$

better than what we need. The second and fourth terms in the above list are estimated similarly. For the third term, perform an additional integration by parts. Either one pulls down an additional factor of at least the decay $\frac{d}{d t}\left[\Psi-\Psi_{\infty}\right]_{1}(t)$, in which case one can integrate absolutely to get the desired upper bound, or ${ }^{54}$ one obtains expressions of the form 55

$$
\int_{T}^{\infty} t(v(t)-1)^{2+a}\left\langle\left(\begin{array}{c}
\tilde{U} \\
\tilde{\tilde{U}}
\end{array}\right)_{\mathrm{dis}}(t, \cdot), \phi\right\rangle d t, \quad a=0,1 .
$$

One can easily handle this by further Duhamel expansion, which we leave out. We now turn to (5.28). This is more difficult, as the strong local dispersive decay rate $\langle s\rangle^{-3 / 2+\delta_{1}}$ combined with 3.14 is not good enough to obtain the desired bound. Then take advantage of the fact that $\dot{v}$ can be written as a convex linear combination of terms $\dot{v}_{i}$, each of which is given by the (derivative of) the right hand side of (3.7) but with right hand side evaluated at a tuple $\Gamma_{i, T} \in A_{[0, T)}^{(9)}$. Again we can focus on the contribution of a single $\dot{v}_{i}$, and by abuse of notation we shall not distinguish between $\Gamma_{i, T}$ and $\Gamma_{T}$, and suppress the subscript $i$. Thus put

$$
\begin{aligned}
& \dot{v}(s)=-b_{\infty} \\
& \quad \cdot \int_{s}^{\infty} \lambda_{\infty}^{-1}(\sigma)\left[v(\sigma)\left(4 i \kappa_{2}\right)^{-1} E_{5}(\sigma)+\beta v(v-1)^{2}(\sigma)+(2 v-1)\left(\beta v-b_{\infty} \lambda_{\infty}^{-1}(\sigma)\right)\right] d \sigma \\
& +v(s)\left(4 i \kappa_{2}\right)^{-1} E_{5}(s)+\beta v(v-1)^{2}(s)+(2 v-1)\left(\beta v-b_{\infty} \lambda_{\infty}^{-1}\right)(s) .
\end{aligned}
$$

We claim that if we replace $\dot{v}$ by the integral expression above, and proceed as in the estimation of (5.27), we obtain an acceptable contribution, i.e., one bounded by $\langle T\rangle^{-1 / 2+\delta_{1}}$.

\footnotetext{
54 In the case when the inner integral gets abolished.

55 Use the decomposition $\left(\begin{array}{c}\tilde{U} \\ \tilde{U}\end{array}\right)=\left(\begin{array}{c}\tilde{U} \\ \tilde{U}\end{array}\right)_{\text {dis }}+\sum_{i=1}^{6} \lambda_{i} \eta_{i, \text { proper}}$; we shall soon see that neither $\lambda_{2}$ nor $\lambda_{6}$ contribute anything to the expression in question, hence the expressions, using 3.18 .
} 
To see this, perform an integration by parts with respect to $s$. If the resulting $s$ derivative falls on the integral expression we substituted for $\dot{v}$, one easily checks, using $\left|E_{5}\right|(s) \lesssim$ $\langle s\rangle^{-2+}$, that the resulting contribution is bounded by $\langle T\rangle^{-1+}$, better than what we need. If the $s$ derivative falls on $\int_{0}^{s} e^{-i \lambda\left(\xi^{2}+1\right)} \mathcal{F}[\ldots] \ldots$, one produces an additional weight decaying at least like $\frac{d}{d s}\left[\Psi-\Psi_{\infty}\right]_{1}(s)$. In this case, repeat integration by parts with respect to $s$, which either kills the integral in the expression we substituted for $\dot{v}$, or we abolish the integral in $\int_{0}^{s} e^{-i \lambda\left(\xi^{2}+1\right)} \mathcal{F}[\ldots] \ldots$ (notice that now the integrand no longer necessarily vanishes at $s=\lambda$ ), or else we produce an extra weight of at least the decay of $\frac{d}{d s}\left[\Psi-\Psi_{\infty}\right]_{1}(s)$. In the first case, we conclude as before, in the second case, one arrives at an expression of essentially the form we started out with at the beginning of (A), but now with weight

$b_{\infty} \int_{s}^{\infty} \lambda_{\infty}^{-1}(\sigma)\left[v(\sigma)\left(4 i \kappa_{2}\right)^{-1} E_{5}(\sigma)+\beta v(v-1)^{2}(\sigma)+(2 v-1)\left(\beta v-b_{\infty} \lambda_{\infty}^{-1}(\sigma)\right)\right] d \sigma$

instead of $v-1$. Then reiterate the whole process started at the beginning of (A), which then leads to easily manageable terms. In the third case, one can close directly by integrating absolutely.

Now we turn to the contribution when $\dot{v}$ in $(5.28)$ is replaced by $v(s)\left(4 i \kappa_{2}\right)^{-1} E_{5}(s)+$ $\beta v(v-1)^{2}(s)+(2 v-1)\left(\beta v-b_{\infty} \lambda_{\infty}^{-1}\right)(s)$. Commence by replacing $\dot{v}(s)$ in 5.28) by $v(s)\left(4 i \kappa_{2}\right)^{-1} E_{5}(s)$. In this case, simple integration by parts with respect to $s$ may be futile, since a priori it is not clear whether the derivative of this term decays faster than $\langle s\rangle^{-2+\delta_{1}+\delta_{3}}$. Now recal[ 56 that

$$
E_{5}=-\left\langle N, \tilde{\xi}_{5}\right\rangle+\left\langle U,\left(i \partial_{s}+\mathcal{H}^{*}(s)\right) \tilde{\xi}_{5}\right\rangle
$$

The first term on the right decays at least as fast as $\langle s\rangle^{-3+2 \delta_{3}}$, and is easily seen to cause no problems. The second term on the right is essentially of the form $(v-1)(s)\left\langle\left(\begin{array}{c}\tilde{U} \\ \tilde{U}\end{array}\right)_{\mathrm{dis}}, \phi\right\rangle$, plus errors which are again negligible. Thus, in order to control 5.28, we need to substitute $(v-1)(s)\left\langle\left(\begin{array}{c}\tilde{U} \\ \tilde{U}\end{array}\right)_{\text {dis }}, \phi\right\rangle$ for $\dot{v}(s)$; our only hope of succeeding here is to Duhamel-expand this second instance of $\left(\begin{array}{c}\tilde{U} \\ \tilde{U}\end{array}\right)_{\text {dis }}$, keeping in mind the iterated nature of the space $A_{[0, T)}^{(10)}$. Pausing in our analysis here for a moment, observe that we run into quite similar issues upon integrating by parts with respect to $s$ in 5.26. Thus combining these contributions, what our problem really boils down to is estimating the expression

$$
\int_{T}^{\infty} t \int_{t}^{\infty}(v-1)(s)\left\langle\left(\begin{array}{c}
\tilde{U} \\
\tilde{\tilde{U}}
\end{array}\right)_{\mathrm{dis}}(s, \cdot), \phi\right\rangle\left\langle\left(\begin{array}{c}
\tilde{U} \\
\tilde{\tilde{U}}
\end{array}\right)_{\mathrm{dis}}(s, \cdot),\left(\mathcal{H}^{*}\right)^{-1} \psi\right\rangle d s d t
$$

where we use schematic notation. Let us assume for now that we can bound this expression by $\langle T\rangle^{-1 / 2+\delta_{1}}$. Then we still need to deal with the case when $\dot{v}(s)$ in $(5.28)$ is replaced by $\beta v(v-1)^{2}(s)+(2 v-1)\left(\beta v-b_{\infty} \lambda_{\infty}^{-1}\right)(s)$ (see the above discussion). The contribution of $\beta v(v-1)^{2}(s)$ is straightforward to handle, using repeated integrations

\footnotetext{
56 See 3.6 .
} 
by parts with respect to $s$, just as before. Finally, substitute $(2 v-1)\left(\beta v-b_{\infty} \lambda_{\infty}^{-1}\right)(s)$ for $\dot{v}(s)$ in 5.28 ). Perform an additional integration by parts in $s$; either one produces an extra factor $\frac{d}{d s}\left[\Psi-\Psi_{\infty}\right]_{1}(s)$, in which case one reiterates integration by parts to arrive either at a much improved expression as in the statement of the lemma, or at an expression which can be integrated absolutely to yield the bound $T^{-1 / 2+\delta_{1}}$. If on the other hand one differentiates $\beta v-b_{\infty} / \lambda_{\infty}$, use (3.8): for example, if the integral in this expression gets hit by $\partial / \partial s$, the worst contribution comes from $E_{S}(s)$, which upon expansion involves a factor $\lambda_{6}(s)$. This one treats by another integration by parts, which either produces an extra $\frac{d}{d s}\left[\Psi-\Psi_{\infty}\right]_{1}(s)$ whence one can integrate absolutely, or else one gets $\dot{\lambda}_{6}(s)$, which is treated by recycling 3.23). There the only dangerous contribution comes from $(v-1)\left\langle\left(\begin{array}{c}\tilde{U} \\ \tilde{U}\end{array}\right)_{\text {dis }}, \phi\right\rangle$, which leads to an expression as in 5.31). The remaining contributions are treated similarly, which up to establishing (5.31) completes treatment of the term (5.28).

We now proceed to estimating 5.29). Reiterate integration by parts in $s$; either one hits $(v-1)(s) \frac{d}{d s}\left[\Psi-\Psi_{\infty}\right]_{1}$, with $\frac{d}{d s}$, or one abolishes the integration in $s$, or one produces at least an extra factor $\frac{d}{d s}\left[\Psi-\Psi_{\infty}\right]_{1}$. In the first case, we obtain an expression as in 5.28 but with an extra weight $v-1$, whence we can treat this case just as above (actually, this time the contribution of $E_{5}$ can just be integrated absolutely). In the third case, reiterate integration by parts, which either takes one into the first two cases, or else produces an additional $\frac{d}{d s}\left[\Psi-\Psi_{\infty}\right]_{1}$. In the last case, keep integrating by parts, which either eventually produces arbitrary gains in $s$, or else takes one into the first two cases. The second case is more tricky: Observe that then we obtain an expression of the form 57

$$
\int_{T}^{\infty} t \int_{t}^{\infty}(v(s)-1) \frac{d}{d s}\left[\Psi-\Psi_{\infty}\right]_{1}(s)\left\langle\left(\begin{array}{c}
\overline{\tilde{U}} \\
\tilde{U}
\end{array}\right)(s, x) \phi(x),\left(\mathcal{H}^{*}\right)^{-2} \phi_{\mathrm{dis}}\right\rangle d s d t .
$$

Observe that a priori the factor $\left(\begin{array}{c}\overline{\tilde{U}} \\ \tilde{U}\end{array}\right)$ might imply the presence of a $\lambda_{6}(s)$, which would lead to an extremely difficult term. However, close inspection of the derivation of the equation for $\lambda_{6}$ shows that in every expression $(v-1)\left\langle\left(\begin{array}{c}\tilde{U} \\ \tilde{U}\end{array}\right)_{\mathrm{dis}}, \phi\right\rangle$ with exactly one power of $v-1$, the (vector-valued) function $\phi$ has the form $\left(\begin{array}{c}\alpha \\ -\alpha\end{array}\right)$ and is real-valued. This then implies that $\phi_{\text {dis }}=\left(\begin{array}{c}\tilde{\alpha} \\ -\tilde{\alpha}\end{array}\right)$, and then also $\left(\mathcal{H}^{*}\right)^{-2} \phi_{\text {dis }}=\left(\begin{array}{c}\beta \\ -\beta\end{array}\right)$, with real-valued scalar function $\beta$. Thus we get $\left\langle\eta_{6, \text { proper }} \phi(x),\left(\begin{array}{c}\beta \\ -\beta\end{array}\right)\right\rangle=0$, and similarly $\left\langle\eta_{2, \operatorname{proper}} \phi(x),\left(\begin{array}{c}\beta \\ -\beta\end{array}\right)\right\rangle=0$, whence

$$
\begin{aligned}
\left\langle\left(\begin{array}{c}
\overline{\tilde{U}} \\
\tilde{U}
\end{array}\right)(s, x) \phi(x),\left(\mathcal{H}^{*}\right)^{-2} \phi_{\text {dis }}\right\rangle \\
=\left\langle\left(\begin{array}{c}
\overline{\tilde{U}} \\
\tilde{U}
\end{array}\right)_{\text {dis }} \phi(x),\left(\mathcal{H}^{*}\right)^{-2} \phi_{\text {dis }}\right\rangle+\sum_{i \neq 2,6}\left\langle\eta_{i, \text { proper }} \phi(x),\left(\mathcal{H}^{*}\right)^{-2} \phi_{\text {dis }}\right\rangle .
\end{aligned}
$$

Thus using 3.18) we have put ourselves into basically the situation we started out with in the lemma (up to negligible error terms), but with a weight $(v-1)(s) \frac{d}{d s}\left[\Psi-\Psi_{\infty}\right]_{1} \sim$

57 The function $\phi(x)$ here is scalar- and real-valued; indeed, one checks that $\phi(x)=\phi_{0}^{4}(x)$. 
$(v-1)^{2}(s)$. Now reiterate the whole process. Observe that performing the same reasoning for the expressions of the form

$$
\int_{T}^{\infty} t \int_{t}^{\infty}(v(s)-1)^{2}\left\langle\left(\begin{array}{c}
\tilde{U} \\
\tilde{U}
\end{array}\right)_{\mathrm{dis}}(s, \cdot) \phi\right\rangle d s d t
$$

which are also implied by 3.23 we may very well arrive at a term of the form

$$
\int_{T}^{\infty} t \int_{t}^{\infty}(v(s)-1)^{2}\left(\frac{d}{d s}\left[\Psi-\Psi_{\infty}\right](s)\right) \lambda_{6}(s) d s d t
$$

Upon integration by part 58 , this leads to

$$
\begin{gathered}
\int_{T}^{\infty} t \int_{t}^{\infty}\left(\int_{s}^{\infty}(v(\sigma)-1)^{3} d \sigma\right) \dot{\lambda}_{6}(s) d s d t, \\
\int_{T}^{\infty} t \int_{t}^{\infty}(v(\sigma)-1)^{3} d \sigma \lambda_{6}(t) d t .
\end{gathered}
$$

The last term is what we started out with in Proposition 5.10, but with an extra factor $\int_{t}^{\infty}(\nu(\sigma)-1)^{3} d \sigma$. Then reiterate the whole process. For the first expression, recycle (3.23); one winds up with terms just as in the lemma, but with the extra weight $\int_{s}^{\infty}(v(\sigma)-$ $1)^{3} d \sigma$. Now reiterate the process. Finally, the term 5.30 is also treated by expanding $\frac{d}{d s}\left[\lambda_{\infty}\left(\mu-\mu_{\infty}\right)\right](s)$ using [3.12). One obtains terms which can either be handled by further integrations by parts in $s$, or else one is led to an expression just as (5.31). As already mentioned, the expression (5.26) is treated by exact analogy. One reiterates the

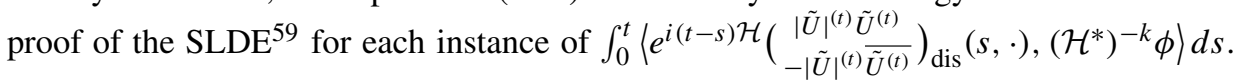
This concludes the proof of the lemma up to the assertion concerning [5.31].

We thus need

Lemma 5.11 60 Under the assumptions of the previous lemma, we have

$$
\left|\int_{T}^{\infty} t \int_{t}^{\infty}(v-1)(s)\left\langle\left(\begin{array}{l}
\tilde{U} \\
\overline{\tilde{U}}
\end{array}\right)_{\mathrm{dis}}(s, \cdot), \phi\right\rangle\left\langle\left(\begin{array}{l}
\tilde{U} \\
\overline{\tilde{U}}
\end{array}\right)_{\mathrm{dis}}(s, \cdot),\left(\mathcal{H}^{*}\right)^{-k} \psi\right\rangle d s d t\right| \lesssim\langle T\rangle^{-1 / 2+\delta_{1}}
$$

for any $k \geq 0$ and Schwartz functions $\phi, \psi$.

Proof. Note that this estimate would be trivial, provided we had the sharp local decay rate $\left|\left\langle\tilde{U}_{\text {dis }}(s), \phi\right\rangle\right| \lesssim s^{-3 / 2}$. Hence our problem consists in eking out a small extra gain. This we do by exploiting some slack in the proof of the strong local dispersive decay. More precisely, what saves us is the fact that the two factors $\left\langle\left(\frac{\tilde{U}}{\tilde{U}}\right)_{\mathrm{dis}}(s, \cdot), \phi\right\rangle$ 'for the most part' oscillate at different frequency with respect to time. Making this precise is unfortunately a bit cumbersome, as we need to unravel the fine structure of these functions by Duhamel-expanding them. By assumption $(\underset{\tilde{U}}{\tilde{U}})_{\text {dis }}$ is a convex linear combination of

\footnotetext{
58 Also, use $\frac{d}{d s}\left[\Psi-\Psi_{\infty}\right]_{1} \sim(v-1)(s)$.

59 Strong local dispersive estimate.

60 The functions $\phi, \psi$ are again generally time dependent in our applications, with derivatives decaying at least like $\dot{v}$. This more general case can be handled just as in the ensuing proof.
} 
functions $\left(\begin{array}{c}\tilde{U} \\ \tilde{U}\end{array}\right)_{i \text {, dis }}$, each of which is obtained as in 3.27). We may then consider the contribution of a single such term, which by abuse of notation we again refer to as $\left(\frac{\tilde{U}}{\tilde{U}}\right)_{\text {dis }}$. We shall also denote the terms on the right hand side of 3.27 by $\left(\begin{array}{c}\tilde{U} \\ \tilde{U}\end{array}\right)_{\text {dis }}$, committing abuse of notation. Then we Duhamel-expand each copy of $\left(\frac{\tilde{U}}{\tilde{U}}\right)_{\text {dis }}(s, \cdot)$ in 5.32 :

$$
\begin{aligned}
& \left(\begin{array}{c}
\tilde{\tilde{U}} \\
\tilde{U}
\end{array}\right)_{\mathrm{dis}}(s, \cdot)=e^{i s \mathcal{H}}\left(\frac{\tilde{U}^{(s)}}{\tilde{U}^{(s)}}\right)_{\mathrm{dis}}(0, \cdot) \\
& \quad+\int_{0}^{s} e^{i(s-\lambda) \mathcal{H}} \\
& \quad \cdot\left[\left(\begin{array}{cc}
0 & 1-e^{2 i\left(\Psi-\Psi_{\infty}\right)_{1}(\lambda)-2 i\left(\Psi-\Psi_{\infty}\right)_{1}(s)} \\
1-e^{-2 i\left(\Psi-\Psi_{\infty}\right)_{1}(\lambda)+2 i\left(\Psi-\Psi_{\infty}\right)_{1}(s)} & 0
\end{array}\right)\left(\frac{\tilde{U}^{(s)}}{\tilde{U}^{(s)}}\right)\right]_{\mathrm{dis}} d \lambda \\
& \quad+\cdots+\int_{0}^{s} e^{i(s-\lambda) \mathcal{H}}\left(\begin{array}{cc}
\left|\tilde{U}^{(s)}\right|^{4} \tilde{U}^{(s)}(\lambda, \cdot) \\
-\left|\tilde{U}^{(s)}\right|^{4} \tilde{\tilde{U}}^{(s)}(\lambda, \cdot)
\end{array}\right)_{\mathrm{dis}} d \lambda:=A+B+\cdots+C .
\end{aligned}
$$

The terms ... are local terms with better decay properties and can be treated in a simpler fashion, hence left out. We then substitute either $A, B$ or $C$ for $\left(\begin{array}{c}\tilde{U} \\ \tilde{U}\end{array}\right)_{\mathrm{dis}}(s, \cdot)$ in 5.32 ) and check that the resulting expression has the same decay as $(v-1)(T)$. The logic behind these estimates is as follows: in an expression of the form

$$
\begin{aligned}
\left\langle e^{i(s-\lambda) \mathcal{H}} E, \phi_{\text {dis }}\right\rangle\left\langle e^{i(s-\lambda) \mathcal{H}} F,\right. & \left.\mathcal{H}^{-k} \psi_{\text {dis }}\right\rangle \\
& =\left\langle E, e^{-i(s-\lambda) \mathcal{H}^{*}} \phi_{\text {dis }}\right\rangle\left\langle F, e^{-i(s-\lambda) \mathcal{H}^{*}}\left(\mathcal{H}^{*}\right)^{-k} \psi_{\text {dis }}\right\rangle,
\end{aligned}
$$

distinguish between the case when $\phi_{\text {dis }}$ and $\psi_{\text {dis }}$ have separated Fourier support, respectively closely aligned (correlated) Fourier support. In the former case, the product oscillates strongly with respect to $s$, whence one can integrate by parts and hope to gain. In the latter case, one should be able to exploit some kind of 'diagonalization effect' in order to gain. The argument proceeds by distinguishing between several interactions:

(AA): This is the expression

$$
\int_{T}^{\infty} t \int_{t}^{\infty}(v-1)(s)\left\langle e^{i s \mathcal{H}}\left(\frac{\tilde{U}^{(s)}}{\tilde{U}^{(s)}}\right)_{\mathrm{dis}}(0, \cdot), \phi\right\rangle\left\langle e^{i s \mathcal{H}}\left(\frac{\tilde{U}^{(s)}}{\tilde{U}^{(s)}}\right)_{\mathrm{dis}}(0, \cdot),\left(\mathcal{H}^{*}\right)^{-k} \psi\right\rangle d s d t
$$

This is straightforward to control upon invoking the spatial localization on the initial data and Theorem 2.1. we get

$$
\begin{array}{r}
\left|\int_{T}^{\infty} t \int_{t}^{\infty}(v-1)(s)\left\langle e^{i s \mathcal{H}}\left(\frac{\tilde{U}^{(s)}}{\tilde{U}^{(s)}}\right)_{\mathrm{dis}}(0, \cdot), \phi\right\rangle\left\langle e^{i s \mathcal{H}}\left(\frac{\tilde{U}^{(s)}}{\tilde{U}^{(s)}}\right)_{\mathrm{dis}}(0, \cdot),\left(\mathcal{H}^{*}\right)^{-k} \psi\right\rangle d s d t\right| \\
\lesssim \int_{T}^{\infty} t \int_{t}^{\infty} s^{-1 / 2+\delta_{1}} s^{-3} d s d t \lesssim T^{-1 / 2+\delta_{1}}
\end{array}
$$


for $\delta_{1}$ small enough, where we use the estimate derived after (5.24), with a trivial modification.

(BB): We employ schematic notation here; scalar quantities really represent vectorial quantities: this is the expression

$$
\begin{gathered}
\int_{T}^{\infty} t \int_{t}^{\infty}(v-1)(s) \int_{0}^{s}\left\langle\left(1-e^{2 i\left(\Psi-\Psi_{\infty}\right)_{1}(\lambda)-2 i\left(\Psi-\Psi_{\infty}\right)_{1}(s)}\right) U^{(s)}(\lambda, \cdot) \phi, e^{-i(s-\lambda) \mathcal{H}^{*}} \phi_{\mathrm{dis}}\right\rangle d \lambda \\
\cdot \int_{0}^{s}\left\langle\left(1-e^{2 i\left(\Psi-\Psi_{\infty}\right)_{1}\left(\lambda^{\prime}\right)-2 i\left(\Psi-\Psi_{\infty}\right)_{1}(s)}\right) U^{(s)}\left(\lambda^{\prime}, \cdot\right) \phi, e^{-i\left(s-\lambda^{\prime}\right) \mathcal{H}^{*}}\left(\mathcal{H}^{*}\right)^{-k} \psi_{\mathrm{dis}}\right\rangle d \lambda^{\prime} d s d t
\end{gathered}
$$

For either of the integrands in the $\lambda$ resp. $\lambda^{\prime}$-integrals one obtains the bound $\lambda^{-3 / 2+\delta_{3}}$ $\cdot(s-\lambda)^{-3 / 2}, \lambda^{\prime-3 / 2+\delta_{3}}\left(s-\lambda^{\prime}\right)^{-3 / 2}$. This clearly suffices as long as $\lambda, \lambda^{\prime}<s / 2$. If for example $\lambda>s / 2$, we can close provided $s-\lambda>s^{10 \delta_{3}}$. In the opposite case, use the inequality

$$
\left|1-e^{2 i\left(\Psi-\Psi_{\infty}\right)_{1}(\lambda)-2 i\left(\Psi-\Psi_{\infty}\right)_{1}(s)}\right|<s^{-1 / 2+\delta_{1}} s^{10 \delta_{3}},
$$

which again allows us to close. The case $(\mathrm{AB})$ is handled similarly.

(BC): This is the expression

$$
\begin{array}{r}
\int_{T}^{\infty} t \int_{t}^{\infty}(v-1)(s) \int_{0}^{s}\left\langle\left(1-e^{2 i\left(\Psi-\Psi_{\infty}\right)_{1}(\lambda)-2 i\left(\Psi-\Psi_{\infty}\right)_{1}(s)}\right) U^{(s)}(\lambda, \cdot) \phi, e^{-i(s-\lambda) \mathcal{H}^{*}} \phi_{\mathrm{dis}}\right\rangle d \lambda \\
\cdot \int_{0}^{s}\left\langle\left(\begin{array}{c}
\left|\tilde{U}^{(s)}\right|^{4} \tilde{U}^{(s)}\left(\lambda^{\prime}, \cdot\right) \\
-\left|\tilde{U}^{(s)}\right|^{4} \tilde{U}^{(s)}\left(\lambda^{\prime}, \cdot\right)
\end{array}\right)_{\mathrm{dis}}, e^{-i\left(s-\lambda^{\prime}\right) \mathcal{H}^{*}}\left(\mathcal{H}^{*}\right)^{-k} \psi_{\mathrm{dis}}\right\rangle d \lambda^{\prime} d s d t
\end{array}
$$

This case is much more difficult. Our method here shall make heavy use of microlocalization. The idea is to first reduce $\phi_{\mathrm{dis}}$ in the local integrand (involving $\lambda$ ) to small $\mathcal{H}$-frequency ${ }^{61}$ Then either $\psi_{\text {dis }}$ is at small frequency, too, which case is handled by exploiting an extra slack in the proof of the strong dispersive estimate, or else there is a gap between the frequency supports of these functions which forces sufficient oscillation in the $s$ variable to render the full expression manageable. First, we observe that we may reduce to $\lambda<s / 2$. This follows from the preceding calculation, since we gain a small power of $s$ in the case $\lambda>s / 2$. Now write

$$
\begin{aligned}
\chi>0 & (x) e^{-i(s-\lambda) \mathcal{H}^{*}} \phi_{\mathrm{dis}} \\
= & \sum_{ \pm} \chi>0(x) \int_{0}^{\infty} e^{ \pm i(s-\lambda)\left(\xi^{2}+1\right)}\left(s(\xi) e^{i x \xi} \underline{e}_{ \pm}+\phi(x, \xi)\right) \tilde{\mathcal{F}}_{ \pm}\left(\phi_{\mathrm{dis}}\right)(\xi) d \xi \\
& +\sum_{ \pm} \chi>0(x) \int_{-\infty}^{0} e^{ \pm i(s-\lambda)\left(\xi^{2}+1\right)}\left[\left(e^{i x \xi}-e^{-i x \xi}\right) \underline{e}_{ \pm}+(1+r(-\xi)) \underline{e}_{ \pm}+\phi(x, \xi)\right] \\
& \cdot \tilde{\mathcal{F}}_{ \pm}\left(\phi_{\mathrm{dis}}\right)(\xi) d \xi
\end{aligned}
$$

61 In the sense that the frequency is close to either 1 or -1 . 
We put $\underline{e}_{+}:=\underline{e}=\left(\begin{array}{l}1 \\ 0\end{array}\right), \underline{e}_{-}:=\sigma_{1} \underline{e}$. Recalling that $\lambda<s / 2$, we claim that we may build in a smooth multiplier $\phi_{<s^{-\epsilon}}(\xi)$ localizing to a dilate of the indicated region $|\xi|<s^{-\epsilon}$ in either integrand, provided $\epsilon>0$ is small enough. Indeed, if on the flip side we build in a multiplier of the form $\phi_{\geq s^{-\epsilon}}(\xi)$, integration by parts in $\xi$ results in arbitrary gains in $s$, at the cost of powers of $x$. These, however, are absorbed by the local factor $U \phi$ above. Thus we shall now replace $\chi_{>0}(x) e^{-i(s-\lambda) \mathcal{H}^{*}} \phi_{\text {dis }}$ by the sum of the above two terms with an extra cutoff $\phi_{<s^{-\epsilon}}(\xi)$ included. Denote this by $\chi>0(x) e^{-i(s-\lambda) \mathcal{H}^{*}} \tilde{\phi}_{\text {dis. }}$. Now we consider the non-local integrand. As above write

$$
\begin{aligned}
& \chi>0(x) e^{-i\left(s-\lambda^{\prime}\right) \mathcal{H}^{*}}\left(\mathcal{H}^{*}\right)^{-k} \psi_{\text {dis }} \\
&= \sum_{ \pm} \chi_{>0}(x) \int_{0}^{\infty} e^{ \pm i\left(s-\lambda^{\prime}\right)\left(\xi^{2}+1\right)}\left(s(\xi) e^{i x \xi} \underline{e}_{ \pm}+\phi(x, \xi)\right) \frac{\tilde{\mathcal{F}}_{ \pm}\left(\psi_{\text {dis }}\right)(\xi)}{\left(\xi^{2}+1\right)^{k}} d \xi \\
& \quad+\sum_{ \pm} \chi>0(x) \int_{-\infty}^{0} e^{ \pm i\left(s-\lambda^{\prime}\right)\left(\xi^{2}+1\right)}\left[\left(e^{i x \xi}-e^{-i x \xi}\right) \underline{e}_{ \pm}+(1+r(-\xi)) \underline{e}_{ \pm}+\phi(x, \xi)\right] \\
& \cdot \frac{\tilde{\mathcal{F}}_{ \pm}\left(\psi_{\text {dis }}\right)(\xi)}{\left(\xi^{2}+1\right)^{k}} d \xi .
\end{aligned}
$$

We then distinguish between the cases $\lambda^{\prime}<s / 2, \lambda^{\prime}>s / 2$.

- $\lambda^{\prime}>s / 2$ : This case is simpler on account of the fact that no extra integration by parts is required to produce the gain of $\left(s-\lambda^{\prime}\right)^{-3 / 2}$ (see the proof of strong local dispersive estimate). The first step consists in reducing $\lambda^{\prime}$ to the range $\left[s / 2, s-s^{1 / 10}\right]$. This follows from the following simple calculation:

$$
\begin{aligned}
& \left|\int_{s-s^{1 / 10}}^{s}\left\langle\left(\begin{array}{c}
\left|\tilde{U}^{(s)}\right|^{4} \tilde{U}^{(s)}\left(\lambda^{\prime}, \cdot\right) \\
-\left|\tilde{U}^{(s)}\right|^{4} \tilde{U}^{(s)}\left(\lambda^{\prime}, \cdot\right)
\end{array}\right), e^{-i\left(s-\lambda^{\prime}\right) \mathcal{H}^{*}}\left(\mathcal{H}^{*}\right)^{-k} \psi_{\mathrm{dis}}\right\rangle d \lambda^{\prime}\right| \\
& \lesssim \int_{s-s^{1 / 10}}^{s}\left\|\tilde{U}^{(s)}\left(\lambda^{\prime}, \cdot\right)\right\|_{L_{x}^{\infty}}^{4}\left\|\tilde{U}^{(s)}\left(\lambda^{\prime}, \cdot\right)\right\|_{L_{x}^{2}}\left\|e^{-i\left(s-\lambda^{\prime}\right) \mathcal{H}^{*}}\left(\mathcal{H}^{*}\right)^{-k} \psi_{\mathrm{dis}}\right\|_{L_{x}^{2}} d \lambda^{\prime} \lesssim s^{1 / 10} s^{-2+\epsilon\left(\delta_{2}\right)},
\end{aligned}
$$

whence we obtain

$$
\begin{array}{r}
\mid \int_{T}^{\infty} t \int_{t}^{\infty}(v-1)(s) \int_{0}^{s}\left\langle\left(1-e^{2 i\left(\Psi-\Psi_{\infty}\right)_{1}(\lambda)-2 i\left(\Psi-\Psi_{\infty}\right)_{1}(s)}\right) U^{(s)}(\lambda, \cdot) \phi, e^{-i(s-\lambda) \mathcal{H}^{*}} \tilde{\phi}_{\mathrm{dis}}\right\rangle d \lambda \\
\cdot \int_{s-s^{1 / 10}}^{s}\left\langle\left(\begin{array}{c}
\left|\tilde{U}^{(s)}\right|^{4} \tilde{U}^{(s)}\left(\lambda^{\prime}, \cdot\right) \\
-\left|\tilde{U}^{(s)}\right|^{4}
\end{array}\right.\right. \\
\left.\left.\quad \lesssim \int^{(s)}\left(\lambda^{\prime}, \cdot\right)\right)_{\mathrm{dis}} t \int_{t}^{-i\left(s-\lambda^{\prime}\right) \mathcal{H}^{*}}\left(\mathcal{H}^{*}\right)^{-k} \psi_{\mathrm{dis}}\right\rangle d \lambda^{\prime} d s d t \mid \\
\quad \int^{-1 / 2+\delta_{1}} s^{-3 / 2} s^{1 / 10} s^{-2+\epsilon\left(\delta_{2}\right)} d s d t \lesssim T^{-1 / 2+\delta_{1}} .
\end{array}
$$

Thus we now reduce to estimating

$$
\begin{array}{r}
\int_{T}^{\infty} t \int_{t}^{\infty}(v-1)(s) \int_{0}^{s / 2}\left\langle\left(1-e^{i\left(\Psi-\Psi_{\infty}\right)_{1}(\lambda)-i\left(\Psi-\Psi_{\infty}\right)_{1}(s)}\right) U^{(s)}(\lambda, \cdot) \phi, e^{-i(s-\lambda) \mathcal{H}^{*}} \tilde{\phi}_{\mathrm{dis}}\right\rangle d \lambda \\
\cdot \int_{s / 2}^{s-s^{1 / 10}}\left\langle\left(\begin{array}{c}
\left|\tilde{U}^{(s)}\right|^{4} \tilde{U}^{(s)}\left(\lambda^{\prime}, \cdot\right) \\
-\left|\tilde{U}^{(s)}\right|^{4} \overline{\tilde{U}^{(s)}}\left(\lambda^{\prime}, \cdot\right)
\end{array}\right)_{\mathrm{dis}}, e^{-i\left(s-\lambda^{\prime}\right) \mathcal{H}^{*}}\left(\mathcal{H}^{*}\right)^{-k} \psi_{\mathrm{dis}}\right\rangle d \lambda^{\prime} d s d t,
\end{array}
$$


where it is to be kept in mind that $e^{-i(s-\lambda) \mathcal{H}^{*}} \tilde{\phi}_{\text {dis }}$ has modified Fourier support as described above. We now mimic the proof of the strong local dispersive estimate (SLDE) for the quintilinear expression. Recall that we rearrange the terms as follows:

$$
\begin{aligned}
\left\langle\left(\begin{array}{c}
\left|\tilde{U}^{(s)}\right|^{4} \tilde{U}^{(s)}\left(\lambda^{\prime}, \cdot\right) \\
-\left|\tilde{U}^{(s)}\right|^{4} \overline{\tilde{U}^{(s)}}\left(\lambda^{\prime}, \cdot\right)
\end{array}\right)_{\mathrm{dis}}, e^{-i\left(s-\lambda^{\prime}\right) \mathcal{H}^{*}}\left(\mathcal{H}^{*}\right)^{-k} \psi_{\mathrm{dis}}\right\rangle \\
=\left\langle\left(\begin{array}{c}
\left|\tilde{U}^{(s)}\right|^{4}\left(\lambda^{\prime}, \cdot\right) \\
-\left|\tilde{U}^{(s)}\right|^{4}\left(\lambda^{\prime}, \cdot\right)
\end{array}\right),\left(\begin{array}{c}
\overline{\tilde{U}^{(s)}}\left(\lambda^{\prime}, \cdot\right) \\
\tilde{U}^{(s)}\left(\lambda^{\prime}, \cdot\right)
\end{array}\right) \times e^{-i\left(s-\lambda^{\prime}\right) \mathcal{H}^{*}}\left(\mathcal{H}^{*}\right)^{-k} \psi_{\mathrm{dis}}\right\rangle .
\end{aligned}
$$

The first step consists in reducing both factors in the preceding expression to their dispersive part: thus write

$$
\begin{aligned}
& \left\langle\left(\begin{array}{c}
\left|\tilde{U}^{(s)}\right|^{4}\left(\lambda^{\prime}, \cdot\right) \\
-\left|\tilde{U}^{(s)}\right|^{4}\left(\lambda^{\prime}, \cdot\right)
\end{array}\right),\left(\begin{array}{c}
\overline{\tilde{U}^{(s)}}\left(\lambda^{\prime}, \cdot\right) \\
\tilde{U}^{(s)}\left(\lambda^{\prime}, \cdot\right)
\end{array}\right) \times e^{-i\left(s-\lambda^{\prime}\right) \mathcal{H}^{*}}\left(\mathcal{H}^{*}\right)^{-k} \psi_{\mathrm{dis}}\right\rangle \\
& =\sum_{i} a_{i}\left\langle\left\langle\left(\begin{array}{c}
\left|\tilde{U}^{(s)}\right|^{4}\left(\lambda^{\prime}, \cdot\right) \\
-\left|\tilde{U}^{(s)}\right|^{4}\left(\lambda^{\prime}, \cdot\right)
\end{array}\right), \xi_{k(i), \text { proper }}\right\rangle \eta_{i, \operatorname{proper}},\left(\begin{array}{c}
\overline{\tilde{U}^{(s)}}\left(\lambda^{\prime}, \cdot\right) \\
\tilde{U}^{(s)}\left(\lambda^{\prime}, \cdot\right)
\end{array}\right) \times e^{-i\left(s-\lambda^{\prime}\right) \mathcal{H}^{*}}\left(\mathcal{H}^{*}\right)^{-k} \psi_{\mathrm{dis}}\right\rangle \\
& \quad+\left\langle\left(\begin{array}{c}
\left|\tilde{U}^{(s)}\right|^{4}\left(\lambda^{\prime}, \cdot\right) \\
-\left|\tilde{U}^{(s)}\right|^{4}\left(\lambda^{\prime}, \cdot\right)
\end{array}\right)_{\mathrm{dis}},\left(\begin{array}{c}
\tilde{U}^{(s)}\left(\lambda^{\prime}, \cdot\right) \\
\tilde{U}^{(s)}\left(\lambda^{\prime}, \cdot\right)
\end{array}\right) \times e^{-i\left(s-\lambda^{\prime}\right) \mathcal{H}^{*}}\left(\mathcal{H}^{*}\right)^{-k} \psi_{\mathrm{dis}}\right\rangle .
\end{aligned}
$$

Note that

$$
\begin{aligned}
&\left.\left|\sum_{i} a_{i}\right|\left\langle\left(\begin{array}{c}
\left|\tilde{U}^{(s)}\right|^{4}\left(\lambda^{\prime}, \cdot\right) \\
-\left|\tilde{U}^{(s)}\right|^{4}\left(\lambda^{\prime}, \cdot\right)
\end{array}\right), \xi_{k(i), \text { proper }}\right\rangle \eta_{i, \operatorname{proper}},\left(\begin{array}{c}
\overline{\tilde{U}^{(s)}}\left(\lambda^{\prime}, \cdot\right) \\
\tilde{U}^{(s)}\left(\lambda^{\prime}, \cdot\right)
\end{array}\right) \times e^{-i\left(s-\lambda^{\prime}\right) \mathcal{H}^{*}}\left(\mathcal{H}^{*}\right)^{-k} \psi_{\mathrm{dis}}\right\rangle \mid \\
& \lesssim \lambda^{\prime-5\left(3 / 2-\delta_{3}\right)}\left(s-\lambda^{\prime}\right)^{-3 / 2}
\end{aligned}
$$

and plugging this back into the above yields an acceptable bound. Thus we now need to estimate

$$
\begin{gathered}
\int_{T}^{\infty} t \int_{t}^{\infty}(v-1)(s) \int_{0}^{s / 2}\left\langle\left(1-e^{2 i\left(\Psi-\Psi_{\infty}\right)_{1}(\lambda)-2 i\left(\Psi-\Psi_{\infty}\right)_{1}(s)}\right) U^{(s)}(\lambda, \cdot) \phi, e^{-i(s-\lambda) \mathcal{H}^{*}} \tilde{\phi}_{\mathrm{dis}}\right\rangle d \lambda \\
\cdot \int_{s / 2}^{s-s^{1 / 10}}\left\langle\left(\begin{array}{c}
\left|\tilde{U}^{(s)}\right|^{4}\left(\lambda^{\prime}, \cdot\right) \\
-\left|\tilde{U}^{(s)}\right|^{4}\left(\lambda^{\prime}, \cdot\right)
\end{array}\right)_{\mathrm{dis}},\left(\begin{array}{c}
\overline{\tilde{U}^{(s)}}\left(\lambda^{\prime}, \cdot\right) \\
\tilde{U}^{(s)}\left(\lambda^{\prime}, \cdot\right)
\end{array}\right) \times e^{-i\left(s-\lambda^{\prime}\right) \mathcal{H}^{*}}\left(\mathcal{H}^{*}\right)^{-k} \psi_{\mathrm{dis}}\right\rangle d \lambda^{\prime} d s d t .
\end{gathered}
$$

Recall from the proof of SLDE that we reformulate

$$
\begin{gathered}
\left\langle\left(\begin{array}{c}
\left|\tilde{U}^{(s)}\right|^{4}\left(\lambda^{\prime}, \cdot\right) \\
-\left|\tilde{U}^{(s)}\right|^{4}\left(\lambda^{\prime}, \cdot\right)
\end{array}\right)_{\mathrm{dis}}, \chi_{>0}(x)\left(\begin{array}{c}
\overline{\tilde{U}^{(s)}}\left(\lambda^{\prime}, \cdot\right) \\
\tilde{U}^{(s)}\left(\lambda^{\prime}, \cdot\right)
\end{array}\right) \times e^{-i\left(s-\lambda^{\prime}\right) \mathcal{H}^{*}}\left(\mathcal{H}^{*}\right)^{-k} \psi_{\mathrm{dis}}\right\rangle \\
=\int_{-\infty}^{\infty} \mathcal{F}\left(\begin{array}{c}
\chi>0(x)\left|\tilde{U}^{(s)}\right|^{4}\left(\lambda^{\prime}, \cdot\right) \\
-\chi_{>0}(x)\left|\tilde{U}^{(s)}\right|^{4}\left(\lambda^{\prime}, \cdot\right)
\end{array}\right)(\xi) \\
\frac{\tilde{\mathcal{F}}\left[\left(\begin{array}{l}
\chi_{>0}(x) \overline{\tilde{U}^{(s)}}\left(\lambda^{\prime}, \cdot\right) \\
\chi_{>0}(x) \tilde{U}^{(s)}\left(\lambda^{\prime}, \cdot\right)
\end{array}\right) \times e^{\left.-i\left(s-\lambda^{\prime}\right) \mathcal{H}^{*}\left(\mathcal{H}^{*}\right)^{-k} \psi_{\mathrm{dis}}\right]}(\xi) d \xi\right.}{(\xi)}
\end{gathered}
$$


Break the integral into the contribution over $[0, \infty)$ and $(-\infty, 0]$. Consider for example the latter, the former being treated similarly. We may write $\mathcal{F}, \tilde{\mathcal{F}}$ purely in terms of the oscillatory part: for example, consider

$$
\begin{aligned}
& \int_{-\infty}^{0} \mathcal{F}\left(\begin{array}{c}
\chi>0(x)\left|\tilde{U}^{(s)}\right|^{4}\left(\lambda^{\prime}, \cdot\right) \\
-\chi>0(x)\left|\tilde{U}^{(s)}\right|^{4}\left(\lambda^{\prime}, \cdot\right)
\end{array}\right)(\xi) \\
& \cdot \overline{\left\langle\left[\left(\begin{array}{l}
\chi_{>0}(x) \overline{\tilde{U}^{(s)}}\left(\lambda^{\prime}, \cdot\right) \\
\chi_{>0}(x) \tilde{U}^{(s)}\left(\lambda^{\prime}, \cdot\right)
\end{array}\right) \times e^{-i\left(s-\lambda^{\prime}\right) \mathcal{H}^{*}}\left(\mathcal{H}^{*}\right)^{-k} \psi_{\mathrm{dis}}\right], \phi(x, \xi)\right\rangle} d \xi .
\end{aligned}
$$

Estimate this by

$$
\begin{aligned}
& \lesssim\left\|\mathcal{F}\left(\begin{array}{c}
\chi_{>0}(x)\left|\tilde{U}^{(s)}\right|^{4}\left(\lambda^{\prime}, \cdot\right) \\
-\chi_{>0}(x)\left|\tilde{U}^{(s)}\right|^{4}\left(\lambda^{\prime}, \cdot\right)
\end{array}\right)\right\|_{L_{\xi}^{2}}
\end{aligned}
$$

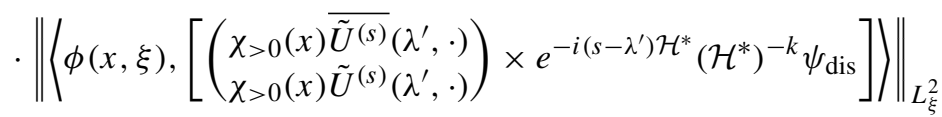

$$
\begin{aligned}
& \lesssim \lambda^{\prime-3 / 2}\left(s-\lambda^{\prime}\right)^{-3 / 2} \text {, }
\end{aligned}
$$

which then leads to an acceptable contribution. One argues similarly for $\mathcal{F}(\ldots)$, and hence ${ }^{62}$ replaces $(5.35)$ by

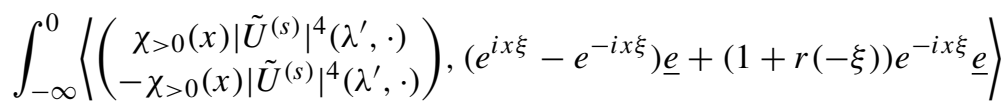

$$
\begin{aligned}
& \cdot \overline{\left\langle\left(\begin{array}{l}
\chi>0 \\
\chi_{>0}(x) \overline{\tilde{U}^{(s)}}\left(\lambda^{\prime}, \cdot\right) \\
\tilde{U}^{(s)}\left(\lambda^{\prime}, \cdot\right)
\end{array}\right) \times e^{-i\left(s-\lambda^{\prime}\right) \mathcal{H}^{*}\left(\mathcal{H}^{*}\right)^{-k}} \psi_{\mathrm{dis}},\left(e^{i x \xi}-e^{-i x \xi}\right) \underline{e}+(1+r(-\xi)) e^{-i x \xi} \underline{e}\right\rangle} d \xi .
\end{aligned}
$$

We first deal with the contributions of the factors $1+r(-\xi)$, which are straightforward: note from the proof of SLDE that we distinguish between the cases $P_{><a}\left[\chi_{>0}(x)\left|\tilde{U}^{(s)}\right|^{2}\right]$ - $P_{><a}\left[\left|\tilde{U}^{(s)}\right|^{2}\right]$, where we put $a=\lambda^{\prime-3 / 4} \sim s^{-3 / 4}$. We then put $a=\lambda^{\prime-3 / 4+\epsilon}$ instead, which leads to a small extra gain in $s$ for all cases except $P_{<a}\left[\chi_{>0}\left|\tilde{U}^{(s)}\right|^{2}\right] P_{<a}\left[\left|\tilde{U}^{(s)}\right|^{2}\right]$. Substituting this forces $|\xi|<s^{-3 / 4+\epsilon}$, and so if either factor $1+r(-\xi)$ occurs we again obtain a gain in $s$ (if $\epsilon$ is small enough). Thus we now reduce to the contribution of

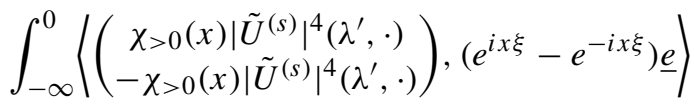

$$
\begin{aligned}
& \cdot\left\langle\left(\begin{array}{l}
\chi_{>0}(x) \overline{\tilde{U}^{(s)}}\left(\lambda^{\prime}, \cdot\right) \\
\chi_{>0}(x) \tilde{U}^{(s)}\left(\lambda^{\prime}, \cdot\right)
\end{array}\right) \times e^{-i\left(s-\lambda^{\prime}\right) \mathcal{H}^{*}}\left(\mathcal{H}^{*}\right)^{-k} \psi_{\mathrm{dis}},\left(e^{i x \xi}-e^{-i x \xi}\right) \underline{e}\right\rangle d \xi
\end{aligned}
$$

Arguing as before, one may reduce this to the expression

\footnotetext{
62 As usual we only consider $\mathcal{F}_{+}$.
} 


$$
\begin{aligned}
& \int_{-\infty}^{0}\left\langle\left(\begin{array}{c}
P_{<a}\left[\chi_{>0}(x)\left|\tilde{U}^{(s)}\right|^{2}\right]\left(\lambda^{\prime}, \cdot\right) P_{<a}\left[\left|\tilde{U}^{(s)}\right|^{2}\right]\left(\lambda^{\prime}, \cdot\right) \\
-P_{<a}\left[\chi_{>0}(x)\left|\tilde{U}^{(s)}\right|^{2}\right]\left(\lambda^{\prime}, \cdot\right) P_{<a}\left[\left|\tilde{U}^{(s)}\right|^{2}\right]\left(\lambda^{\prime}, \cdot\right)
\end{array}\right),\left(e^{i x \xi}-e^{-i x \xi}\right) \underline{e}\right\rangle \\
& \cdot \overline{\left.\left(\begin{array}{l}
\chi_{>0}(x) \overline{\tilde{U}^{(s)}}\left(\lambda^{\prime}, \cdot\right) \\
\chi_{>0}(x) \tilde{U}^{(s)}\left(\lambda^{\prime}, \cdot\right)
\end{array}\right) \times e^{-i\left(s-\lambda^{\prime}\right) \mathcal{H}^{*}\left(\mathcal{H}^{*}\right)^{-k}} \psi_{\mathrm{dis}},\left(e^{i x \xi}-e^{-i x \xi}\right) \underline{e}\right\rangle} d \xi,
\end{aligned}
$$

where $a=\lambda^{\prime-3 / 4+\epsilon} \sim s^{-3 / 4+\epsilon}$. Still following the proof of SLDE, we expand

$$
\begin{aligned}
\chi>0 & (x) e^{-i\left(s-\lambda^{\prime}\right) \mathcal{H}^{*}}\left(\mathcal{H}^{*}\right)^{-k} \psi_{\mathrm{dis}} \\
=\chi>0 & (x) \sum_{ \pm} \int_{0}^{\infty} e^{ \pm i\left(s-\lambda^{\prime}\right)\left(\xi^{2}+1\right)}\left(s(\xi) e^{i x \xi} \underline{e}_{ \pm}+\phi_{ \pm}(x, \xi)\right) \frac{\mathcal{F}_{ \pm}\left(\psi_{\mathrm{dis}}\right)(\xi)}{\left(\xi^{2}+1\right)^{k}} d \xi \\
+\chi_{>0}(x) \sum_{ \pm} \int_{-\infty}^{0} e^{ \pm i\left(s-\lambda^{\prime}\right)\left(\xi^{2}+1\right)} & \cdot\left(\left[e^{i x \xi}-e^{-i x \xi}\right] \underline{e}_{ \pm}+(1+r(-\xi)) e^{-i x \xi} \underline{e}_{ \pm}+\phi_{ \pm}(x, \xi)\right) \frac{\mathcal{F}_{ \pm}\left(\psi_{\mathrm{dis}}\right)(\xi)}{\left(\xi^{2}+1\right)^{k}} d \xi
\end{aligned}
$$

We shall localize $\xi$ here away from 0 . For example, consider the following expression:

$$
\int_{-\infty}^{0} e^{ \pm i\left(s-\lambda^{\prime}\right)\left(\xi^{2}+1\right)}\left[e^{i x \xi}-e^{-i x \xi}\right] \underline{e}_{ \pm} \frac{\mathcal{F}_{ \pm}\left(\psi_{\mathrm{dis}}\right)(\xi)}{\left(\xi^{2}+1\right)^{k}} d \xi=\frac{e^{ \pm i\left(s-\lambda^{\prime}\right)}}{\sqrt{s-\lambda^{\prime}}} \int_{-\infty}^{\infty} e^{\mp \frac{(x-y)^{2}}{i\left(s-\lambda^{\prime}\right)}} g(y) d y
$$

where $g(y)=\int_{-\infty}^{0}\left[e^{i y \xi}-e^{-i y \xi}\right] \underline{e}_{ \pm} \frac{\mathcal{F}_{ \pm}\left(\psi_{\text {dis }}\right)(\xi)}{\left(\xi^{2}+1\right)^{k}} d \xi$. It is straightforward to see that we may include a smooth multiplier $\phi_{<s} 1000(\xi)$ here. With this modification we then have $|g(y)| \lesssim\langle y\rangle^{-2} \log s$, whence ${ }^{63}$ we may replace $g(y)$ by

$$
\phi_{<s^{1 / 10}}(y) \int_{-\infty}^{0} \phi_{<s^{1000}}(\xi)\left[e^{i y \xi}-e^{-i y \xi}\right] \underline{e}_{ \pm} \frac{\mathcal{F}_{ \pm}\left(\psi_{\mathrm{dis}}\right)(\xi)}{\left(\xi^{2}+1\right)^{k}} d \xi
$$

We now claim that we may further localize $\xi$ away from 0 . For this include a sharp cutoff $\chi_{<s}-\epsilon_{1}(\xi)$, i.e., consider the contribution of

$$
\tilde{g}(y):=\phi_{<s^{1 / 10}}(y) \int_{-s^{-\epsilon_{1}}}^{0} \phi_{<s^{1000}}(\xi)\left[e^{i y \xi}-e^{-i y \xi}\right] \underline{e}_{ \pm} \frac{\mathcal{F}_{ \pm}\left(\psi_{\mathrm{dis}}\right)(\xi)}{\left(\xi^{2}+1\right)^{k}} d \xi .
$$

We integrate by parts here, and replace this expression by

$$
\begin{aligned}
\phi_{<s^{1 / 10}}(y) \frac{e^{i y s^{-\epsilon_{1}}}+e^{-i y s^{-\epsilon_{1}}}}{i y} \underline{e}_{ \pm} \frac{\mathcal{F}_{ \pm}\left(\psi_{\mathrm{dis}}\right)\left(-s^{-\epsilon_{1}}\right)}{\left(s^{-2 \epsilon_{1}}+1\right)^{k}} \\
-\phi_{<s^{1 / 10}}(y) \int_{-s^{-\epsilon_{1}}}^{0} \frac{\left[e^{i y \xi}+e^{-i y \xi}\right]}{i y} \underline{e}_{ \pm} \partial_{\xi}\left[\phi_{<s^{1000}}(\xi) \frac{\mathcal{F}_{ \pm}\left(\psi_{\mathrm{dis}}\right)(\xi)}{\left(\xi^{2}+1\right)^{k}}\right] d \xi
\end{aligned}
$$

63 Following the proof of SLDE. 
One now easily verifies that $\|\tilde{g}(y)\|_{L_{y}^{1}} \lesssim s^{-\epsilon_{1}} \log s$, which extra gain suffices to close all the other estimates upon reiterating the proof of SLDE. We now see that we may replace $g(y)$ by the expression

$$
h(y):=\phi_{<s^{1 / 10}}(y) \int_{-\infty}^{-s^{-\epsilon_{1}}} \phi_{<s^{1000}}(\xi)\left[e^{i y \xi}-e^{-i y \xi}\right] \underline{e}_{ \pm} \frac{\mathcal{F}_{ \pm}\left(\psi_{\mathrm{dis}}\right)(\xi)}{\left(\xi^{2}+1\right)^{k}} d \xi .
$$

The Heisenberg uncertainty principle implies that this function has frequency $\gtrsim s^{-\epsilon_{1}}$ (for $\left.\epsilon_{1} \ll 1 / 10\right)$ up to errors of size $s^{-N}$, and hence negligible, and the same comment applies to the function

$$
\frac{e^{ \pm i\left(s-\lambda^{\prime}\right)}}{\sqrt{s-\lambda^{\prime}}} \int_{-\infty}^{\infty} e^{\mp \frac{(x-y)^{2}}{i\left(s-\lambda^{\prime}\right)}} h(y) d y=\int_{-\infty}^{\infty} e^{ \pm i\left(s-\lambda^{\prime}\right)\left(\xi^{\prime 2}+1\right)} e^{i x \xi^{\prime}} \hat{h}\left(\xi^{\prime}\right) d \xi^{\prime} .
$$

Thus we may replace ${ }^{64}$ this function by the following, where $\phi_{\geq s^{-\epsilon_{1}}}$ is a smooth cutoff localizing to $\left|\xi^{\prime}\right| \gtrsim s^{-\epsilon_{1}}$ :

$$
\int_{-\infty}^{\infty} e^{ \pm i\left(s-\lambda^{\prime}\right)\left(\xi^{\prime 2}+1\right)} e^{i x \xi^{\prime}} \phi_{\geq s^{-\epsilon_{1}}}\left(\xi^{\prime}\right) \hat{h}\left(\xi^{\prime}\right) d \xi^{\prime}
$$

Recalling 5.36 we now need to estimate the contribution of

$$
\begin{aligned}
& \int_{-\infty}^{0}\left\langle\left(\begin{array}{c}
P_{<a}\left[\chi_{>0}(x)\left|\tilde{U}^{(s)}\right|^{2}\right]\left(\lambda^{\prime}, \cdot\right) P_{<a}\left[\left|\tilde{U}^{(s)}\right|^{2}\right]\left(\lambda^{\prime}, \cdot\right) \\
-P_{<a}\left[\chi_{>0}(x)\left|\tilde{U}^{(s)}\right|^{2}\right]\left(\lambda^{\prime}, \cdot\right) P_{<a}\left[\left|\tilde{U}^{(s)}\right|^{2}\right]\left(\lambda^{\prime}, \cdot\right)
\end{array}\right),\left(e^{i x \xi}-e^{-i x \xi}\right) \underline{e}\right\rangle
\end{aligned}
$$

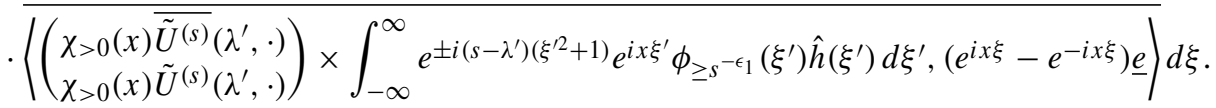

This then gets substituted for

$$
\left\langle\left(\begin{array}{c}
\left|\tilde{U}^{(s)}\right|^{4}\left(\lambda^{\prime}, \cdot\right) \\
-\left|\tilde{U}^{(s)}\right|^{4}\left(\lambda^{\prime}, \cdot\right)
\end{array}\right)_{\operatorname{dis}},\left(\begin{array}{c}
\overline{\tilde{U}^{(s)}}\left(\lambda^{\prime}, \cdot\right) \\
\tilde{U}^{(s)}\left(\lambda^{\prime}, \cdot\right)
\end{array}\right) \times e^{-i\left(s-\lambda^{\prime}\right) \mathcal{H}^{*}}\left(\mathcal{H}^{*}\right)^{-k} \psi_{\mathrm{dis}}\right\rangle
$$

in 5.33 . It is now important to recall that in 5.33 we already reduced $e^{-i(s-\lambda) \mathcal{H}^{*}} \tilde{\phi}_{\text {dis }}$ to frequency $<s^{-\epsilon}$ (see the paragraph before $(5.33)$ ). Thus writing $e^{-i(s-\lambda) \mathcal{H}^{*}} \tilde{\phi}_{\text {dis }}$ in terms of its Fourier expansion with variable $\xi$ and rearranging exponentials we get the phase $e^{i s\left(\xi^{2}-\xi^{\prime 2}\right)}$ in the case of destructive resonance (and $e^{i s\left(\xi^{2}+\xi^{\prime 2}+2\right)}$ for constructive resonance). If we arrange $\epsilon_{1} \gg \epsilon$, as we may, we have $\left|\xi^{2}-\xi^{\prime 2}\right| \gtrsim s^{-\epsilon_{1}}$. Then switch orders of integration in (5.33), first performing an integration with respect to $s$. This costs $s^{\epsilon_{1}}$ but either demolishes the integral in $\lambda^{\prime}$ or else produces at least extra factors $\partial_{s}\left[\lambda_{\infty}\left[\mu-\mu_{\infty}\right]\right](s), \frac{d}{d s}\left(\Psi-\Psi_{\infty}\right)_{1} \sim(v-1)(s)$. In order to decouple the variables $\xi, \xi^{\prime}$, notice that for $\left|\xi^{\prime}\right| \lesssim s^{\epsilon}$ we have 65

$$
\phi_{<s^{-\epsilon}}(\xi) \phi_{\geq s^{-\epsilon_{1}}}\left(\xi^{\prime}\right) \frac{e^{i\left(\xi^{2}-\xi^{\prime 2}\right) s}}{\xi^{2}-\xi^{\prime 2}}=\sum_{n, m \in s^{\epsilon} \mathbb{Z}^{2}} e^{i\left(\xi^{2}-\xi^{\prime 2}\right) s} a_{n, m} e^{i n \xi+i m \xi^{\prime}},
$$

64 Generating negligible error terms.

65 We may easily reduce to $\left|\xi^{\prime}\right| \lesssim s^{\epsilon}$, see e.g. the argument for Lemma 5.12 
where $\sum_{n, m}[|n|+|m|]^{C}\left|a_{n, m}\right| \lesssim s^{C \epsilon_{1}}$. If one substitutes this back into (5.33) and proceeds as in the proof of SLDE, one gets an extra gain in $s$ upon choosing $\epsilon_{1}$ small enough, as desired. In detail, consider

$$
\begin{aligned}
& \int_{T}^{\infty} t \int_{t}^{\infty}(v-1)(s) \int_{0}^{s / 2}\left\langle\left(1-e^{2 i\left(\Psi-\Psi_{\infty}\right)_{1}(\lambda)-2 i\left(\Psi-\Psi_{\infty}\right)_{1}(s)}\right) U^{(s)}(\lambda, \cdot) \phi, e^{-i(s-\lambda) \mathcal{H}^{*}} \tilde{\phi}_{\mathrm{dis}}\right\rangle d \lambda \\
& \cdot \int_{s / 2}^{s-s^{1 / 10}} \int_{-\infty}^{0}\left\langle\left(\begin{array}{c}
P_{<a}\left[\chi_{>0}(x)\left|\tilde{U}^{(s)}\right|^{2}\right]\left(\lambda^{\prime}, \cdot\right) P_{<a}\left[\left|\tilde{U}^{(s)}\right|^{2}\right]\left(\lambda^{\prime}, \cdot\right) \\
-P_{<a}\left[\chi_{>0}(x)\left|\tilde{U}^{(s)}\right|^{2}\right]\left(\lambda^{\prime}, \cdot\right) P_{<a}\left[\left|\tilde{U}^{(s)}\right|^{2}\right]\left(\lambda^{\prime}, \cdot\right)
\end{array}\right),\left(e^{i x \xi}-e^{-i x \xi}\right) \underline{e}\right\rangle
\end{aligned}
$$

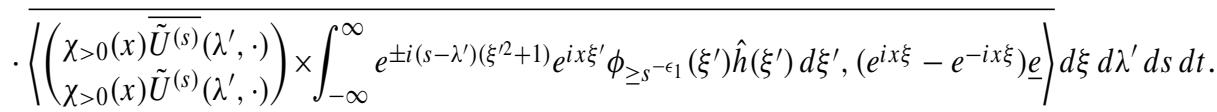

Then substitute 5.34 with a suitable frequency cutoff as discussed there, which amongst similar terms results in

$$
\begin{aligned}
& \int_{T}^{\infty} t \int_{t}^{\infty}(v-1)(s) \int_{0}^{s / 2}\left\langle\left(1-e^{2 i\left(\Psi-\Psi_{\infty}\right)_{1}(\lambda)-2 i\left(\Psi-\Psi_{\infty}\right)_{1}(s)}\right) U^{(s)}(\lambda, \cdot) \phi,\right. \\
& \left.\int_{-\infty}^{0} e^{ \pm i(s-\lambda)\left(\eta^{2}+1\right)} \phi_{<s^{-\epsilon}}(\eta)\left(e^{i x \eta}-e^{-i x \eta}\right) \mathcal{F}\left(\phi_{\mathrm{dis}}\right) \eta\right\rangle d \lambda \\
& \cdot \int_{s / 2}^{s-s^{1 / 10}} \int_{-\infty}^{0}\left\langle\left(\begin{array}{c}
P_{<a}\left[\chi>0(x)\left|\tilde{U}^{(s)}\right|^{2}\right]\left(\lambda^{\prime}, \cdot\right) P_{<a}\left[\left|\tilde{U}^{(s)}\right|^{2}\right]\left(\lambda^{\prime}, \cdot\right) \\
-P_{<a}\left[\chi_{>0}(x)\left|\tilde{U}^{(s)}\right|^{2}\right]\left(\lambda^{\prime}, \cdot\right) P_{<a}\left[\left|\tilde{U}^{(s)}\right|^{2}\right]\left(\lambda^{\prime}, \cdot\right)
\end{array}\right),\left(e^{i x \xi}-e^{-i x \xi}\right) \underline{e}\right\rangle \\
& \cdot \overline{\left\langle\left(\begin{array}{l}
\chi_{>0}(x) \overline{\tilde{U}^{(s)}}\left(\lambda^{\prime}, \cdot\right) \\
\chi_{>0}(x) \tilde{U}^{(s)}\left(\lambda^{\prime}, \cdot\right)
\end{array}\right) \times \int_{-\infty}^{\infty} e^{ \pm i\left(s-\lambda^{\prime}\right)\left(\xi^{\prime 2}+1\right)} e^{i x \xi^{\prime}} \phi_{\geq s^{-\epsilon_{1}}}\left(\xi^{\prime}\right) \hat{h}\left(\xi^{\prime}\right) d \xi^{\prime},\left(e^{i x \xi}-e^{-i x \xi}\right) \underline{e}\right\rangle} d \xi d \lambda^{\prime} d s d t .
\end{aligned}
$$

Rewrite this as

$$
\int_{T}^{\infty} t \int_{t}^{\infty}(v-1)(s) \int_{-\infty}^{0} \int_{-\infty}^{0} \int_{-\infty}^{\infty} \int_{0}^{\infty} \int_{0}^{\infty} e^{i s\left( \pm \eta^{2} \pm \xi^{2}\right)} \Psi\left(s, \lambda, \lambda^{\prime}, \eta, \xi, \xi^{\prime}\right) d \lambda d \lambda^{\prime} d \xi^{\prime} d \eta d \xi d s d t
$$

Now perform an integration by parts in $s$, after switching the orders of integration, then restore the original order of integration:

$$
\begin{aligned}
& \int_{T}^{\infty} t \int_{t}^{\infty}(v-1)(s) \int_{-\infty}^{0} \int_{-\infty}^{0} \int_{-\infty}^{\infty} \int_{0}^{\infty} \int_{0}^{\infty} e^{i s\left( \pm \eta^{2} \pm \xi^{\prime 2}\right)} \Psi\left(s, \lambda, \lambda^{\prime}, \eta, \xi, \xi^{\prime}\right) d \eta d \xi d \xi^{\prime} d \lambda d \lambda^{\prime} d s d t \\
& =-\int_{T}^{\infty} t(v-1)(t) \int_{-\infty}^{0} \int_{-\infty}^{0} \int_{-\infty}^{\infty} \int_{0}^{\infty} \int_{0}^{\infty} \frac{e^{i t\left( \pm \eta^{2} \pm \xi^{\prime 2}\right)}}{i\left( \pm \eta^{2} \pm \xi^{\prime 2}\right)} \Psi\left(t, \lambda, \lambda^{\prime}, \eta, \xi, \xi^{\prime}\right) d \eta d \xi d \xi^{\prime} d \lambda d \lambda^{\prime} d s d t \\
& +\int_{T}^{\infty} t \int_{t}^{\infty} \dot{v}(s) \int_{-\infty}^{0} \int_{-\infty}^{0} \int_{-\infty}^{\infty} \int_{0}^{\infty} \int_{0}^{\infty} \frac{e^{i s\left( \pm \eta^{2} \pm \xi^{2}\right)}}{i\left( \pm \eta^{2} \pm \xi^{\prime 2}\right)} \Psi\left(s, \lambda, \lambda^{\prime}, \eta, \xi, \xi^{\prime}\right) d \eta d \xi d \xi^{\prime} d \lambda d \lambda^{\prime} d s d t \\
& +\int_{T}^{\infty} t \int_{t}^{\infty}(v-1)(s) \int_{-\infty}^{0} \int_{-\infty}^{0} \int_{-\infty}^{\infty} \int_{0}^{\infty} \int_{0}^{\infty} \frac{e^{i s\left( \pm \eta^{2} \pm \xi^{\prime 2}\right)}}{i\left( \pm \eta^{2} \pm \xi^{\prime 2}\right)} \partial_{s} \Psi\left(s, \lambda, \lambda^{\prime}, \eta, \xi, \xi^{\prime}\right) d \eta d \xi d \xi^{\prime} d \lambda d \lambda^{\prime} d s d t
\end{aligned}
$$

We have $\left|\xi^{\prime 2} \pm \eta^{2}\right| \gtrsim s^{-\epsilon_{1}}$ on the support of each integrand. If one then decouples the variables $\xi^{\prime}, \eta$ as outlined above and then proceeds as in the proof of SLDE, one checks 
that each of these terms can be bounded by $\lesssim T^{-1 / 2+\delta_{1}-\mu\left(\epsilon_{1}\right)}$ upon choosing $\epsilon_{1}$ small enough, which suffices. This concludes the case $\lambda^{\prime}>s / 2$.

- $\lambda^{\prime}<s / 2$, still in case (BC): This is the expression

$$
\begin{aligned}
\int_{T}^{\infty} t & \int_{t}^{\infty}(v-1)(s) \int_{0}^{s / 2}\left\langle\left(1-e^{2 i\left(\Psi-\Psi_{\infty}\right)_{1}(\lambda)-2 i\left(\Psi-\Psi_{\infty}\right)_{1}(s)}\right) U^{(s)}(\lambda, \cdot) \phi, e^{-i(s-\lambda) \mathcal{H}^{*}} \tilde{\phi}_{\mathrm{dis}}\right\rangle d \lambda \\
\cdot & \int_{0}^{s / 2}\left\langle\left(\begin{array}{c}
\left|\tilde{U}^{(s)}\right|^{4}\left(\lambda^{\prime}, \cdot\right) \\
-\left|\tilde{U}^{(s)}\right|^{4}\left(\lambda^{\prime}, \cdot\right)
\end{array}\right),\left(\begin{array}{c}
\overline{\tilde{U}^{(s)}}\left(\lambda^{\prime}, \cdot\right) \\
\tilde{U}^{(s)}\left(\lambda^{\prime}, \cdot\right)
\end{array}\right) \times e^{-i\left(s-\lambda^{\prime}\right) \mathcal{H}^{*}}\left(\mathcal{H}^{*}\right)^{-k} \psi_{\mathrm{dis}}\right\rangle d \lambda^{\prime} d s d t .
\end{aligned}
$$

We start again by reducing

$$
\left\langle\chi>0\left(\begin{array}{c}
\left|\tilde{U}^{(s)}\right|^{4}\left(\lambda^{\prime}, \cdot\right) \\
-\left|\tilde{U}^{(s)}\right|^{4}\left(\lambda^{\prime}, \cdot\right)
\end{array}\right),\left(\begin{array}{c}
\overline{\tilde{U}^{(s)}}\left(\lambda^{\prime}, \cdot\right) \\
\tilde{U}^{(s)}\left(\lambda^{\prime}, \cdot\right)
\end{array}\right) \times e^{-i\left(s-\lambda^{\prime}\right) \mathcal{H}^{*}}\left(\mathcal{H}^{*}\right)^{-k} \psi_{\mathrm{dis}}\right\rangle
$$

to

$$
\left\langle\chi>0\left(\begin{array}{c}
\left|\tilde{U}^{(s)}\right|^{4}\left(\lambda^{\prime}, \cdot\right) \\
-\left|\tilde{U}^{(s)}\right|^{4}(\lambda, \cdot)
\end{array}\right)_{\mathrm{dis}},\left(\begin{array}{c}
\overline{\tilde{U}^{(s)}}\left(\lambda^{\prime}, \cdot\right) \\
\tilde{U}^{(s)}\left(\lambda^{\prime}, \cdot\right)
\end{array}\right) \times e^{-i\left(s-\lambda^{\prime}\right) \mathcal{H}^{*}}\left(\mathcal{H}^{*}\right)^{-k} \psi_{\mathrm{dis}}\right\rangle .
$$

This follows from

$$
\begin{aligned}
\sum_{i} \mid\left\langle\chi>0\left\langle\left(\begin{array}{c}
\left|\tilde{U}^{(s)}\right|^{4} \\
-\left|\tilde{U}^{(s)}\right|^{4}
\end{array}\right), \xi_{i}\right\rangle \eta_{i},\left(\begin{array}{c}
\overline{\tilde{U}^{(s)}}\left(\lambda^{\prime}, \cdot\right) \\
\tilde{U}^{(s)}\left(\lambda^{\prime}, \cdot\right)
\end{array}\right) \times\right. & \left.e^{-i\left(s-\lambda^{\prime}\right) \mathcal{H}^{*}}\left(\mathcal{H}^{*}\right)^{-k} \psi_{\mathrm{dis}}\right\rangle \mid \\
& \lesssim \lambda^{\prime-5\left(3 / 2-\delta_{3}\right)}\left(s-\lambda^{\prime}\right)^{-3 / 2}
\end{aligned}
$$

Now replace 5.39 by

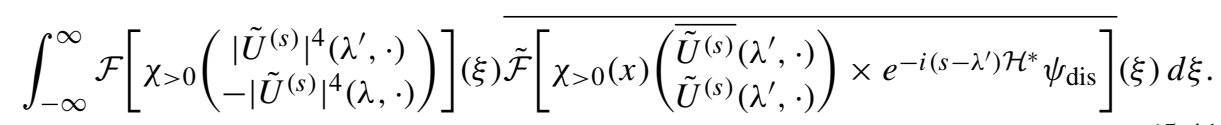

We shall again simplify the Fourier transform here: for example, consider the contribution of

$$
\begin{aligned}
& \int_{-\infty}^{\infty}\left\langle\chi>0\left(\begin{array}{c}
\left|\tilde{U}^{(s)}\right|^{4}\left(\lambda^{\prime}, \cdot\right) \\
-\left|\tilde{U}^{(s)}\right|^{4}(\lambda, \cdot)
\end{array}\right), \phi(x, \xi)\right\rangle \\
& \overline{\tilde{\mathcal{F}}\left[\chi>0(x)\left(\begin{array}{c}
\overline{\tilde{U}^{(s)}}\left(\lambda^{\prime}, \cdot\right) \\
\tilde{U}^{(s)}\left(\lambda^{\prime}, \cdot\right)
\end{array}\right) \times e^{-i\left(s-\lambda^{\prime}\right) \mathcal{H}^{*}}\left(\mathcal{H}^{*}\right)^{-k} \psi_{\mathrm{dis}}\right]}(\xi) d \xi .
\end{aligned}
$$

Proceeding as in the proof of SLDE one bounds this by $\lesssim \lambda^{\prime-4\left(3 / 2-\delta_{3}\right)} \lambda^{\prime}\left(s-\lambda^{\prime}\right)^{-3 / 2}$, which is more than enough. Further, for example the contribution of

$$
\begin{array}{r}
\int_{-\infty}^{0}\left\langle\chi>0\left(\begin{array}{c}
\left|\tilde{U}^{(s)}\right|^{4}\left(\lambda^{\prime}, \cdot\right) \\
-\left|\tilde{U}^{(s)}\right|^{4}(\lambda, \cdot)
\end{array}\right),(1+r(-\xi)) e^{-i x \xi} \underline{e}\right\rangle \\
\cdot \frac{\tilde{\mathcal{F}}\left[\chi_{>0}(x)\left(\begin{array}{c}
\overline{\tilde{U}^{(s)}}\left(\lambda^{\prime}, \cdot\right) \\
\tilde{U}^{(s)}\left(\lambda^{\prime}, \cdot\right)
\end{array}\right) \times e^{-i\left(s-\lambda^{\prime}\right) \mathcal{H}^{*}\left(\mathcal{H}^{*}\right)^{-k}} \psi_{\mathrm{dis}}\right]}{(\xi) d \xi}
\end{array}
$$


is treated as in the case $\lambda^{\prime}>s / 2$ (one does not gain in $s$ but in $\lambda^{\prime}$ ). Thus focusing on the more difficult reflection part, we need to estimate the contribution of

$$
\begin{aligned}
& \int_{-\infty}^{0}\left\langle\chi>0\left(\begin{array}{c}
\left|\tilde{U}^{(s)}\right|^{4}\left(\lambda^{\prime}, \cdot\right) \\
-\left|\tilde{U}^{(s)}\right|^{4}(\lambda, \cdot)
\end{array}\right),\left(e^{i x \xi}-e^{-i x \xi}\right) \underline{e}\right\rangle \\
& \cdot \overline{\left\langle\left[\chi_{>0}(x)\left(\begin{array}{c}
\overline{\tilde{U}^{(s)}}\left(\lambda^{\prime}, \cdot\right) \\
\tilde{U}^{(s)}\left(\lambda^{\prime}, \cdot\right)
\end{array}\right) \times e^{-i\left(s-\lambda^{\prime}\right) \mathcal{H}^{*}\left(\mathcal{H}^{*}\right)^{-k}} \psi_{\mathrm{dis}}\right],\left(e^{i x \xi}-e^{-i x \xi}\right) \underline{e}\right\rangle} d \xi .
\end{aligned}
$$

Arguing as in the case $\lambda^{\prime}>s / 2$, we may reduce this expression further to

$$
\begin{aligned}
& \int_{-\infty}^{0}\left\langle\left(\begin{array}{c}
P_{<a}\left[\chi_{>0}\left|\tilde{U}^{(s)}\right|^{2}\right]\left(\lambda^{\prime}, \cdot\right) P_{<a}\left[\left|\tilde{U}^{(s)}\right|^{2}\right]\left(\lambda^{\prime}, \cdot\right) \\
-P_{<a}\left[\chi_{>0}\left|\tilde{U}^{(s)}\right|^{2}\right]\left(\lambda^{\prime}, \cdot\right) P_{<a}\left[\left|\tilde{U}^{(s)}\right|^{2}\right]\left(\lambda^{\prime}, \cdot\right)
\end{array}\right),\left(e^{i x \xi}-e^{-i x \xi}\right) \underline{e}\right\rangle \\
& \cdot \overline{\left\langle\left[\chi_{>0}(x)\left(\begin{array}{c}
\overline{\tilde{U}^{(s)}}\left(\lambda^{\prime}, \cdot\right) \\
\tilde{U}^{(s)}\left(\lambda^{\prime}, \cdot\right)
\end{array}\right) \times e^{-i\left(s-\lambda^{\prime}\right) \mathcal{H}^{*}}\left(\mathcal{H}^{*}\right)^{-k} \psi_{\mathrm{dis}}\right],\left(e^{i x \xi}-e^{-i x \xi}\right) \underline{e}\right\rangle} d \xi
\end{aligned}
$$

where $a=\lambda^{\prime-3 / 4+\epsilon}$. We shall next show that we may localize the Fourier support of the term $e^{-i\left(s-\lambda^{\prime}\right) \mathcal{H}^{*}}\left(\mathcal{H}^{*}\right)^{-k} \psi_{\text {dis }}$ away from zero, in which case we can conclude as in the case $\lambda^{\prime}>s / 2$, exploiting the frequency separation in order to perform an integration by parts in $s$. Recall from the proof of the SLDE that in this case, we need to perform an extra integration by parts in the frequency variable in order to obtain the gain $\left(s-\lambda^{\prime}\right)^{-3 / 2}$. More precisely, in the expression

$$
\left\langle\chi>0\left(\begin{array}{c}
\left|\tilde{U}^{(s)}\right|^{4} \\
-\left|\tilde{U}^{(s)}\right|^{4}
\end{array}\right)_{\mathrm{dis}},\left(\begin{array}{c}
\overline{\tilde{U}^{(s)}}\left(\lambda^{\prime}, \cdot\right) \\
\tilde{U}^{(s)}\left(\lambda^{\prime}, \cdot\right)
\end{array}\right) \times e^{-i\left(s-\lambda^{\prime}\right) \mathcal{H}^{*}}\left(\mathcal{H}^{*}\right)^{-k} \psi_{\mathrm{dis}}\right\rangle,
$$

we replace the expression

$$
\chi>0(x)\left(\begin{array}{c}
\overline{\tilde{U}^{(s)}}\left(\lambda^{\prime}, \cdot\right) \\
\tilde{U}^{(s)}\left(\lambda^{\prime}, \cdot\right)
\end{array}\right) \times e^{-i\left(s-\lambda^{\prime}\right) \mathcal{H}^{*}}\left(\mathcal{H}^{*}\right)^{-k} \psi_{\mathrm{dis}}
$$

by

$$
\begin{aligned}
& \chi>0(x)\left(\begin{array}{c}
\overline{\tilde{U}^{(s)}}\left(\lambda^{\prime}, \cdot\right) \\
x \tilde{U}^{(s)}\left(\lambda^{\prime}, \cdot\right)
\end{array}\right) \times \frac{1}{s-\lambda^{\prime}} \int_{-\infty}^{0} e^{-i\left(s-\lambda^{\prime}\right)\left(\xi^{2}+1\right)}\left(e^{i x \xi}+e^{-i x \xi}\right) \underline{e}\left[\frac{\mathcal{F}\left(\psi_{\mathrm{dis}}\right)(\xi)}{\left(\xi^{2}+1\right)^{k} \xi}\right] d \xi, \\
& \chi>0(x)\left(\begin{array}{c}
\overline{\tilde{U}^{(s)}}\left(\lambda^{\prime}, \cdot\right) \\
\tilde{U}^{(s)}\left(\lambda^{\prime}, \cdot\right)
\end{array}\right) \times \frac{1}{s-\lambda^{\prime}} \int_{-\infty}^{0} e^{-i\left(s-\lambda^{\prime}\right)\left(\xi^{2}+1\right)}\left(e^{i x \xi}-e^{-i x \xi}\right) \underline{e} \partial \xi\left[\frac{\mathcal{F}\left(\psi_{\mathrm{dis}}\right)(\xi)}{\left(\xi^{2}+1\right)^{k} \xi}\right] d \xi, \\
& \chi>0(x)\left(\begin{array}{c}
\overline{\tilde{U}^{(s)}}\left(\lambda^{\prime}, \cdot\right) \\
x \tilde{U}^{(s)}\left(\lambda^{\prime}, \cdot\right)
\end{array}\right) \times \frac{1}{s-\lambda^{\prime}} \int_{0}^{\infty} e^{-i\left(s-\lambda^{\prime}\right)\left(\xi^{2}+1\right)} s(\xi) e^{i x \xi} \underline{e} \frac{\mathcal{F}\left(\psi_{\mathrm{dis}}\right)(\xi)}{\left(\xi \xi^{2}+1\right)^{k} \xi} d \xi, \\
& \chi>0(x)\left(\begin{array}{c}
\left(\tilde{U}^{(s)}\left(\lambda^{\prime}, \cdot\right)\right. \\
\tilde{U}^{(s)}\left(\lambda^{\prime}, \cdot\right)
\end{array}\right) \times \frac{1}{s-\lambda^{\prime}} \int_{0}^{\infty} e^{-i\left(s-\lambda^{\prime}\right)\left(\xi^{2}+1\right)} \partial \xi s(\xi) e^{i x \xi} \frac{\mathcal{F}\left(\psi_{\mathrm{dis}}\right)(\xi)}{\left(\xi^{2}+1\right)^{k} \xi} d \xi,
\end{aligned}
$$


as well as the expressions

$$
\begin{aligned}
& \chi_{>0}(x)\left(\begin{array}{l}
\overline{\tilde{U}^{(s)}}\left(\lambda^{\prime}, \cdot\right) \\
\tilde{U}^{(s)}\left(\lambda^{\prime}, \cdot\right)
\end{array}\right) \times \frac{1}{s-\lambda^{\prime}} \int_{-\infty}^{0} e^{-i\left(s-\lambda^{\prime}\right)\left(\xi^{2}+1\right)} e^{-i x \xi} \partial_{\xi}[1+r(-\xi)] \underline{e}\left[\frac{\mathcal{F}\left(\psi_{\mathrm{dis}}\right)(\xi)}{\left(\xi^{2}+1\right)^{k} \xi}\right] d \xi, \\
& \chi_{>0}(x)\left(\begin{array}{l}
\overline{\tilde{U}^{(s)}}\left(\lambda^{\prime}, \cdot\right) \\
\tilde{U}^{(s)}\left(\lambda^{\prime}, \cdot\right)
\end{array}\right) \times \frac{1}{s-\lambda^{\prime}} \int_{-\infty}^{0} e^{-i\left(s-\lambda^{\prime}\right)\left(\xi^{2}+1\right)} \partial_{\xi}\left[\phi(x, \xi)\left[\frac{\mathcal{F}\left(\psi_{\mathrm{dis}}\right)(\xi)}{\left(\xi^{2}+1\right)^{k} \xi}\right]\right] d \xi,
\end{aligned}
$$

as well as similar terms which can be treated identically. The last term but one here is equivalent to the last term but two for all intents and purposes. Moreover, the last term can be treated by the same argument as for the last term but two, so we shall now consider the four terms after and including 5.45. Start with 5.45): we have

$$
\begin{aligned}
\int_{-\infty}^{0} e^{-i\left(s-\lambda^{\prime}\right)\left(\xi^{2}+1\right)}\left(e^{i x \xi}+e^{-i x \xi}\right) \underline{e}\left[\frac{\mathcal{F}\left(\psi_{\mathrm{dis}}\right)(\xi)}{\left(\xi^{2}+1\right)^{k} \xi}\right] & d \xi \\
& =\frac{1}{\sqrt{s-\lambda^{\prime}}} \int_{-\infty}^{\infty} e^{\frac{(x-y)^{2}}{i\left(s-\lambda^{\prime}\right)}} g(y) d y
\end{aligned}
$$

where $g(y)=\int_{-\infty}^{0}\left(e^{i y \xi}+e^{-i y \xi}\right) \underline{e}\left[\frac{\mathcal{F}\left(\phi_{\text {dis }}\right)(\xi)}{\left(\xi^{2}+1\right)^{k \xi}}\right] d \xi$ satisfies $|g(y)| \lesssim\langle y\rangle^{-2}$, whence we may replace it by $\tilde{g}(y)=\phi_{<\lambda^{\prime 1 / 10}}(y) g(y)$. Now we specialize this further and consider the contribution of

$$
h(y):=\phi_{<\lambda^{\prime \prime} / 10}(y) \int_{-\infty}^{0} \phi_{<\lambda^{\prime} \epsilon_{1}}(\xi)\left(e^{i y \xi}+e^{-i y \xi}\right) \underline{e}\left[\frac{\mathcal{F}\left(\phi_{\mathrm{dis}}\right)(\xi)}{\left(\xi^{2}+1\right)^{k} \xi}\right] d \xi .
$$

By the Heisenberg principle, it has frequency in the interval $\left[0, \lambda^{\prime-\epsilon_{1} / 2}\right]$ up to errors of size $\lambda^{\prime-N}$, which we may neglect. Now consider the bracket

$$
\left\langle\left(e^{i x \xi}-e^{-i x \xi}\right) \underline{e},\left[\chi_{>0}(x)\left(\begin{array}{c}
\overline{\tilde{U}^{(s)}}\left(\lambda^{\prime}, \cdot\right) \\
x \tilde{U}^{(s)}\left(\lambda^{\prime}, \cdot\right)
\end{array}\right) \times \frac{1}{\left(\sqrt{s-\lambda^{\prime}}\right)^{3}} \int_{-\infty}^{\infty} e^{(x-y)^{2} / i\left(s-\lambda^{\prime}\right)} h(y) d y\right]\right\rangle,
$$

where we have the restriction $|\xi| \lesssim \lambda^{\prime-3 / 4+\epsilon}$. We can then rewrite this as

$$
\begin{aligned}
& \chi \leq \lambda^{\prime-3 / 4+\epsilon}(\xi)\left\langle\left(e^{i x \xi}-e^{-i x \xi}\right) \underline{e},\right. \\
& \left.\left[P_{<\lambda^{\prime} \epsilon_{1} / 2}\left[\left(\begin{array}{c}
\chi>0 \\
\chi>0 \\
\chi>0) x(x) \tilde{U}^{(s)}\left(\lambda^{\prime}, \cdot\right) \\
\left(\lambda^{\prime}, \cdot\right)
\end{array}\right)\right] \times \frac{1}{\left(\sqrt{s-\lambda^{\prime}}\right)^{3}} \int_{-\infty}^{\infty} e^{\frac{(x-y)^{2}}{i\left(s-\lambda^{\prime}\right)}} h(y) d y\right]\right) .
\end{aligned}
$$

Then we use Littlewood-Paley dichotomy in order to get

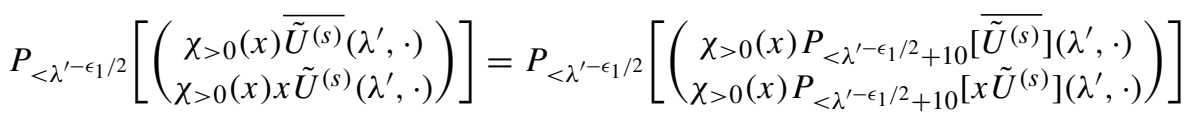

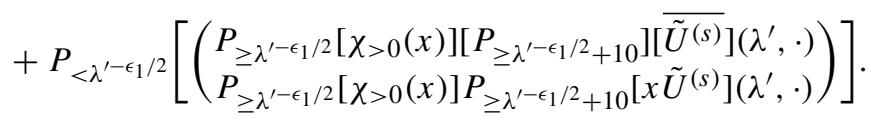


We first consider the contribution from the second term on the right. We substitute this back into (5.44), undo the Fourier transform using Plancherel's Theorem, and estimate this by

$$
\begin{aligned}
& \left.\lesssim\left\|\chi>0\left(\begin{array}{c}
P_{<a}\left[\chi_{>0}\left|\tilde{U}^{(s)}\right|^{2}\right]\left(\lambda^{\prime}, \cdot\right) P_{<a}\left[\left|\tilde{U}^{(s)}\right|^{2}\right]\left(\lambda^{\prime}, \cdot\right) \\
-P_{<a}\left[\chi_{>0}\left|\tilde{U}^{(s)}\right|^{2}\right]\left(\lambda^{\prime}, \cdot\right) P_{<a}\left[\left|\tilde{U}^{(s)}\right|^{2}\right]\left(\lambda^{\prime}, \cdot\right)
\end{array}\right)\right\|_{L_{x}^{\infty}} \| P_{\geq \lambda^{\prime-\epsilon_{1} / 2}[\chi>0}(x)\right] \|_{L_{x}^{1}} \\
& \cdot\left\|x \tilde{U}^{(s)}\left(\lambda^{\prime}, \cdot\right)\right\|_{L_{x}^{\infty}}\left\|\frac{1}{\left(\sqrt{s-\lambda^{\prime}}\right)^{3}} \int_{-\infty}^{\infty} e^{\frac{(x-y)^{2}}{i\left(s-\lambda^{\prime}\right)}} h(y) d y\right\|_{L_{x}^{\infty}} \\
& \lesssim \lambda^{\prime-3 / 2+\epsilon_{1}}\left(s-\lambda^{\prime}\right)^{-3 / 2},
\end{aligned}
$$

which is then seen to lead to an acceptable contribution upon substitution into 5.37). Thus we may now focus on the contribution of

$$
\begin{aligned}
\left\langle\left(e^{i x \xi}-e^{-i x \xi}\right) \underline{e},\right. \\
\left.\left.\qquad \chi \chi>0(x)\left(\begin{array}{c}
P_{<\lambda^{\prime} \epsilon_{1} / 2+10}\left[\overline{\tilde{U}^{(s)}}\right]\left(\lambda^{\prime}, \cdot\right) \\
P_{<\lambda^{\prime-\epsilon} / 2+10}\left[x \tilde{U}^{(s)}\right]\left(\lambda^{\prime}, \cdot\right)
\end{array}\right) \times \frac{1}{\left(\sqrt{s-\lambda^{\prime}}\right)^{3}} \int_{-\infty}^{\infty} e^{\frac{(x-y)^{2}}{i\left(s-\lambda^{\prime}\right)}} h(y) d y\right]\right),
\end{aligned}
$$

always keeping in mind that $|\xi|<\lambda^{\prime-3 / 4+\epsilon}$. We now replicate the proof of SLDE for the low-low case (keep in mind that the full expression we estimate is (5.44)). Thus we write $\left(p=-i \partial_{x}\right)$

$P_{<\lambda^{\prime-\epsilon_{1} / 2+10}}\left[x \tilde{U}^{(s)}\right](t, \cdot)=P_{<\lambda^{\prime-\epsilon_{1} / 2+10}}\left[(x+2 i p t) \tilde{U}^{(s)}\right](t, \cdot)-P_{<\lambda^{\prime-\epsilon_{1} / 2+10}}\left[2 i p t \tilde{U}^{(s)}\right](t, \cdot)$.

Then we have

$$
\left[i \partial_{t}+\Delta\right] P_{<\lambda^{\prime-\epsilon_{1} / 2+10}} \nabla U(t, \cdot)=P_{<\lambda^{\prime-\epsilon_{1} / 2+10}} \nabla\left[V U+\cdots+|U|^{4} U\right]
$$

just as in the proof of the strong local dispersive estimate. Now one further manipulates the expressions on the right just as in the proof of SLDE. Note that the operator $P_{<\lambda^{\prime} \epsilon_{1} / 2+10} \nabla$ will smear out the supports a bit, but this is easily seen to be harmless. Of course one gains $\lambda^{\prime-\epsilon_{1} / 2}$ in the process, which overcomes any small losses in the proof of SLDE. One can now restrict to $|\xi|>\lambda^{\prime-\epsilon_{1}}$, i.e., include a multiplier $\phi_{\geq \lambda^{\prime-\epsilon_{1}}}$ in the definition of $h(y)$, and then finish the argument just as in the case $\lambda^{\prime}>s / 2$. This concludes estimating the contribution from the term 5.45 . The contribution of the third term in that list is treated analogously. We now turn to the contribution of the second term there, i.e., the expression

$$
\begin{aligned}
& \left\langle\chi>0\left(\begin{array}{c}
\left|\tilde{U}^{(s)}\right|^{4} \\
-\left|\tilde{U}^{(s)}\right|^{4}
\end{array}\right)_{\mathrm{dis}}\left(\lambda^{\prime}, \cdot\right),\right. \\
& \left.\chi>0(x)\left(\begin{array}{c}
\overline{\tilde{U}^{(s)}}\left(\lambda^{\prime}, \cdot\right) \\
\tilde{U}^{(s)}\left(\lambda^{\prime}, \cdot\right)
\end{array}\right) \times \frac{1}{s-\lambda^{\prime}} \int_{-\infty}^{0} e^{-i\left(s-\lambda^{\prime}\right)\left(\xi^{2}+1\right)}\left(e^{i x \xi}-e^{-i x \xi}\right) \underline{e} \partial \xi\left[\frac{\mathcal{F}\left(\psi_{\mathrm{dis}}\right)(\xi)}{\left(\xi^{2}+1\right)^{k} \xi}\right] d \xi\right\rangle .
\end{aligned}
$$

But this is easily seen to be estimable by

$$
\lesssim \lambda^{\prime-3 / 2}\left(s-\lambda^{\prime}\right)^{-3 / 2},
$$


which upon substitution into (5.37) yields an acceptable contribution. The fourth term after (5.45) is handled analogously. We are done with the case (BC). Clearly the case (AC) can be handled analogously.

(CC): The most difficult case. This is the expression

$$
\begin{aligned}
\int_{T}^{\infty} t \int_{t}^{\infty}(v-1)(s) \int_{0}^{s}\left\langle\left(\begin{array}{c}
\left|\tilde{U}^{(s)}\right|^{4} \tilde{U}^{(s)}(\lambda, \cdot) \\
-\left|\tilde{U}^{(s)}\right|^{4} \tilde{U}^{(s)}(\lambda, \cdot)
\end{array}\right)_{\mathrm{dis}}, e^{-i(s-\lambda) \mathcal{H}^{*}} \phi_{\mathrm{dis}}\right\rangle d \lambda \\
\quad \times \int_{0}^{s}\left\langle\left(\begin{array}{c}
\left|\tilde{U}^{(s)}\right|^{4} \tilde{U}^{(s)}\left(\lambda^{\prime}, \cdot\right) \\
-\left|\tilde{U}^{(s)}\right|^{4} \tilde{U}^{(s)}\left(\lambda^{\prime}, \cdot\right)
\end{array}\right)_{\mathrm{dis}}, e^{-i\left(s-\lambda^{\prime}\right) \mathcal{H}^{*}}\left(\mathcal{H}^{*}\right)^{-k} \psi_{\mathrm{dis}}\right\rangle d \lambda^{\prime} d s d t .
\end{aligned}
$$

Start with the case $\max \left\{\lambda, \lambda^{\prime}\right\}<s / 2$. We may restrict integration to the range $\lambda>\lambda^{\prime}$. Rearrange either of the factors in the integral as

$$
\begin{aligned}
& \left\langle\chi>0\left(\begin{array}{c}
\left|\tilde{U}^{(s)}\right|^{4}\left(\lambda^{\prime}, \cdot\right) \\
-\left|\tilde{U}^{(s)}\right|^{4}\left(\lambda^{\prime}, \cdot\right)
\end{array}\right),\left(\begin{array}{c}
\overline{\tilde{U}^{(s)}}\left(\lambda^{\prime}, \cdot\right) \\
\tilde{U}^{(s)}\left(\lambda^{\prime}, \cdot\right)
\end{array}\right) \times e^{-i\left(s-\lambda^{\prime}\right) \mathcal{H}^{*}}\left(\mathcal{H}^{*}\right)^{-k} \psi_{\mathrm{dis}}\right\rangle, \\
& \left\langle\chi>0\left(\begin{array}{c}
\left|\tilde{U}^{(s)}\right|^{4}(\lambda, \cdot) \\
-\left|\tilde{U}^{(s)}\right|^{4}(\lambda, \cdot)
\end{array}\right),\left(\begin{array}{c}
\overline{\tilde{U}^{(s)}(\lambda, \cdot)} \\
\tilde{U}^{(s)}(\lambda, \cdot)
\end{array}\right) \times e^{-i(s-\lambda) \mathcal{H}^{*}} \phi_{\mathrm{dis}}\right\rangle .
\end{aligned}
$$

As usual, we first need to reduce both factors in either bracket to their dispersive part. This time, though, we have to analyze each constituent more carefully, since they all interact with each other. Thus we now write

$$
\begin{aligned}
\chi_{>0}\left(\begin{array}{c}
\left|\tilde{U}^{(s)}\right|^{4}\left(\lambda^{\prime}, \cdot\right) \\
-\left|\tilde{U}^{(s)}\right|^{4}\left(\lambda^{\prime}, \cdot\right)
\end{array}\right) & =\sum_{i} a_{i}\left\langle\chi_{>0}\left(\begin{array}{c}
\left|\tilde{U}^{(s)}\right|^{4}\left(\lambda^{\prime}, \cdot\right) \\
-\left|\tilde{U}^{(s)}\right|^{4}\left(\lambda^{\prime}, \cdot\right)
\end{array}\right), \xi_{k(i)}\right) \eta_{i}+\left(\begin{array}{c}
\left|\chi_{>0} \tilde{U}^{(s)}\right|^{4}\left(\lambda^{\prime}, \cdot\right) \\
-\chi_{>0}\left|\tilde{U}^{(s)}\right|^{4}\left(\lambda^{\prime}, \cdot\right)
\end{array}\right) \text { dis } \\
& :=\alpha+\beta .
\end{aligned}
$$

First consider the contribution from $\alpha\left(\lambda^{\prime}, \cdot\right)$, i.e., the expression

$$
\left\langle\alpha\left(\lambda^{\prime}, \cdot\right),\left(\begin{array}{c}
\overline{\tilde{U}^{(s)}}\left(\lambda^{\prime}, \cdot\right) \\
\tilde{U}^{(s)}\left(\lambda^{\prime}, \cdot\right)
\end{array}\right) \times e^{-i\left(s-\lambda^{\prime}\right) \mathcal{H}^{*}}\left(\mathcal{H}^{*}\right)^{-k} \psi_{\mathrm{dis}}\right\rangle
$$

As usual, we expand

$$
e^{-i\left(s-\lambda^{\prime}\right) \mathcal{H}^{*}}\left(\mathcal{H}^{*}\right)^{-k} \psi_{\mathrm{dis}}=\sum_{ \pm} \int_{-\infty}^{\infty} e^{ \pm i\left(s-\lambda^{\prime}\right)\left(\xi^{2}+1\right)} e_{ \pm}(x, \xi) \frac{\mathcal{F}_{ \pm}\left(\psi_{\mathrm{dis}}\right)}{\left(\xi^{2}+1\right)^{k}} d \xi
$$

We claim that we may sneak in a smooth cutoff $\phi_{<s^{-\epsilon}}(\xi)$ into this integrand, which we then denote as $e^{-i\left(s-\lambda^{\prime}\right) \mathcal{H}^{*}}\left(\mathcal{H}^{*}\right)^{-k} \tilde{\psi}_{\text {dis }}$. This is because integration by parts in $\xi$ costs in addition to $s^{\epsilon}$ at most $\max \{|x|, \xi\}$, and for $\alpha$ we may assume $|x|$ to be bounded, whence choosing $\epsilon$ small enough results in a gain in $s$. Of course we use $\lambda^{\prime}<s / 2$ here. The same comment applies to $\alpha(\lambda, \cdot)$. Our strategy will be to achieve a localization away from zero for the frequencies of $e^{-i(s-\lambda) \mathcal{H}^{*}} \phi_{\mathrm{dis}}, e^{-i(s-\lambda) \mathcal{H}^{*}}\left(\mathcal{H}^{*}\right)^{-k} \psi_{\mathrm{dis}}$, occurring in the contribution from $\beta(\lambda, \cdot), \beta\left(\lambda^{\prime}, \cdot\right)$. This ensures that $\alpha\left(\lambda^{\prime}, \cdot\right)$ and $\beta(\lambda, \cdot)$ etc. interact weakly. We now distinguish between the following cases: 
$(\alpha \alpha)$ : This is the expression

$$
\begin{gathered}
\int_{T}^{\infty} t \int_{t}^{\infty}(v-1)(s) \int_{0}^{s / 2}\left\langle\left\langle\chi>0\left(\begin{array}{c}
\left|\tilde{U}^{(s)}\right|^{4}(\lambda, \cdot) \\
-\left|\tilde{U}^{(s)}\right|^{4}(\lambda, \cdot)
\end{array}\right), \xi_{i}\right\rangle \eta_{i},\left(\begin{array}{c}
\overline{\tilde{U}^{(s)}}(\lambda, \cdot) \\
\tilde{U}^{(s)}(\lambda, \cdot)
\end{array}\right) \times e^{-i(s-\lambda) \mathcal{H}^{*}} \tilde{\phi}_{\mathrm{dis}}\right\rangle d \lambda \\
\cdot \int_{0}^{s / 2}\left\langle\left\langle\chi>\left(\begin{array}{c}
\left|\tilde{U}^{(s)}\right|^{4}\left(\lambda^{\prime}, \cdot\right) \\
-\left|\tilde{U}^{(s)}\right|^{4}\left(\lambda^{\prime}, \cdot\right)
\end{array}\right), \xi_{i}\right\rangle \eta_{i},\left(\begin{array}{c}
\left(\tilde{U}^{(s)}\left(\lambda^{\prime}, \cdot\right)\right. \\
\tilde{U}^{(s)}\left(\lambda^{\prime}, \cdot\right)
\end{array}\right) \times e^{-i\left(s-\lambda^{\prime}\right) \mathcal{H}^{*}}\left(\mathcal{H}^{*}\right)^{-k} \tilde{\psi}_{\mathrm{dis}}\right\rangle d \lambda^{\prime} d s d t .
\end{gathered}
$$

We can easily estimate this by

$\lesssim \int_{T}^{\infty} t \int_{t}^{\infty} s^{-1 / 2+\delta_{1}} \int_{0}^{s / 2} \lambda^{-5\left(3 / 2-\delta_{3}\right)}(s-\lambda)^{-3 / 2} d \lambda \int_{0}^{s / 2} \lambda^{\prime-5\left(3 / 2-\delta_{3}\right)}\left(s-\lambda^{\prime}\right)^{-3 / 2} d \lambda^{\prime} d s d t$ $\lesssim T^{-1 / 2+\delta_{1}}$.

$(\alpha \beta)$ : This is the expression

$$
\begin{aligned}
& \int_{T}^{\infty} t \int_{t}^{\infty}(v-1)(s) \int_{0}^{s / 2}\left\langle\left\langle\chi>0\left(\begin{array}{c}
\left|\tilde{U}^{(s)}\right|^{4}(\lambda, \cdot) \\
-\left|\tilde{U}^{(s)}\right|^{4}(\lambda, \cdot)
\end{array}\right), \xi_{i}\right\rangle \eta_{i},\left(\begin{array}{c}
\overline{\tilde{U}^{(s)}}(\lambda, \cdot) \\
\tilde{U}^{(s)}(\lambda, \cdot)
\end{array}\right) \times e^{-i(s-\lambda) \mathcal{H}^{*}} \tilde{\phi}_{\mathrm{dis}}\right\rangle d \lambda \\
& \cdot \int_{0}^{s / 2}\left\langle\left(\begin{array}{c}
\chi>0\left|\tilde{U}^{(s)}\right|^{4}\left(\lambda^{\prime}, \cdot\right) \\
-\chi>0\left|\tilde{U}^{(s)}\right|^{4}\left(\lambda^{\prime}, \cdot\right)
\end{array}\right)_{\mathrm{dis}},\left(\begin{array}{c}
\overline{\tilde{U}^{(s)}}\left(\lambda^{\prime}, \cdot\right) \\
\tilde{U}^{(s)}\left(\lambda^{\prime}, \cdot\right)
\end{array}\right) \times e^{-i\left(s-\lambda^{\prime}\right) \mathcal{H}^{*}}\left(\mathcal{H}^{*}\right)^{-k} \psi_{\mathrm{dis}}\right\rangle d \lambda^{\prime} d s d t .
\end{aligned}
$$

To proceed, we restrict the frequency of $e^{-i\left(s-\lambda^{\prime}\right) \mathcal{H}^{*}} \psi_{\text {dis }}$ away from 0 . The procedure for this is identical to the one outlined in case (BC). Having achieved frequency separation, we have of course achieved rapid oscillation in $s$, whence we can close this case as at the end of case (BC), by integration by parts in $s$. The case $(\beta \alpha)$ is handled analogously.

$(\beta \beta)$ : This is the expression

$$
\begin{aligned}
& \int_{T}^{\infty} t \int_{t}^{\infty}(v-1)(s) \int_{0}^{s / 2}\left\langle\left(\begin{array}{c}
\chi>0\left|\tilde{U}^{(s)}\right|^{4}(\lambda, \cdot) \\
-\chi>0\left|\tilde{U}^{(s)}\right|^{4}(\lambda, \cdot)
\end{array}\right)_{\mathrm{dis}},\left(\begin{array}{c}
\overline{\tilde{U}^{(s)}}(\lambda, \cdot) \\
\tilde{U}^{(s)}(\lambda, \cdot)
\end{array}\right) \times e^{-i(s-\lambda) \mathcal{H}^{*}} \phi_{\mathrm{dis}}\right\rangle d \lambda \\
& \cdot \int_{0}^{s / 2}\left\langle\left(\begin{array}{c}
\chi>0\left|\tilde{U}^{(s)}\right|^{4}\left(\lambda^{\prime}, \cdot\right) \\
-\chi>0\left|\tilde{U}^{(s)}\right|^{4}\left(\lambda^{\prime}, \cdot\right)
\end{array}\right)_{\mathrm{dis}},\left(\begin{array}{c}
\overline{\tilde{U}^{(s)}}\left(\lambda^{\prime}, \cdot\right) \\
\tilde{U}^{(s)}\left(\lambda^{\prime}, \cdot\right)
\end{array}\right) \times e^{-i\left(s-\lambda^{\prime}\right) \mathcal{H}^{*}}\left(\mathcal{H}^{*}\right)^{-k} \psi_{\mathrm{dis}}\right\rangle d \lambda^{\prime} d s d t .
\end{aligned}
$$

As before we mimic the proof of SLDE. Thus we perform an integration by parts in the Fourier representation for $e^{-i(s-\lambda) \mathcal{H}^{*}} \phi_{\text {dis }}$ etc. and produce the following list of terms provided the integration by parts results in a loss of $x$. Call this list $\beta_{1}$ :

$$
\chi>0(x)\left(\begin{array}{c}
\overline{\tilde{U}^{(s)}}\left(\lambda^{\prime}, \cdot\right) \\
x \tilde{U}^{(s)}\left(\lambda^{\prime}, \cdot\right)
\end{array}\right) \times \frac{1}{s-\lambda^{\prime}} \int_{-\infty}^{0} e^{-i\left(s-\lambda^{\prime}\right)\left(\xi^{2}+1\right)}\left(e^{i x \xi}+e^{-i x \xi}\right) \underline{e}\left[\frac{\mathcal{F}\left(\psi_{\mathrm{dis}}\right)(\xi)}{\left(\xi^{2}+1\right)^{k} \xi}\right] d \xi,
$$




$$
\begin{aligned}
& \chi>0(x)\left(\begin{array}{c}
\overline{\tilde{U}^{(s)}}\left(\lambda^{\prime}, \cdot\right) \\
x \tilde{U}^{(s)}\left(\lambda^{\prime}, \cdot\right)
\end{array}\right) \times \frac{1}{s-\lambda^{\prime}} \int_{0}^{\infty} e^{-i\left(s-\lambda^{\prime}\right)\left(\xi^{2}+1\right)} s(\xi) e^{i x \xi} \underline{e} \frac{\mathcal{F}\left(\psi_{\mathrm{dis}}\right)(\xi)}{\left(\xi^{2}+1\right)^{k} \xi} d \xi \\
& \chi>0(x)\left(\begin{array}{c}
\overline{\tilde{U}^{(s)}(\lambda, \cdot)} \\
x \tilde{U}^{(s)}(\lambda, \cdot)
\end{array}\right) \times \frac{1}{s-\lambda} \int_{-\infty}^{0} e^{-i(s-\lambda)\left(\xi^{2}+1\right)}\left(e^{i x \xi}+e^{-i x \xi}\right) \underline{e}\left[\frac{\mathcal{F}\left(\phi_{\mathrm{dis}}\right)(\xi)}{\xi}\right] d \xi \\
& \chi>0(x)\left(\begin{array}{c}
\overline{\tilde{U}^{(s)}(\lambda, \cdot)} \\
x \tilde{U}^{(s)}(\lambda, \cdot)
\end{array}\right) \times \frac{1}{s-\lambda} \int_{0}^{\infty} e^{-i(s-\lambda)\left(\xi^{2}+1\right)} s(\xi) e^{i x \xi} \underline{e} \frac{\mathcal{F}\left(\phi_{\mathrm{dis}}\right)(\xi)}{\xi} d \xi
\end{aligned}
$$

These get complemented by the following terms, which we refer to as $\beta_{2}$ :

$$
\begin{aligned}
& \chi_{>0}(x)\left(\begin{array}{c}
\overline{\tilde{U}^{(s)}}\left(\lambda^{\prime}, \cdot\right) \\
\tilde{U}^{(s)}\left(\lambda^{\prime}, \cdot\right)
\end{array}\right) \times \frac{1}{s-\lambda^{\prime}} \int_{-\infty}^{0} e^{-i\left(s-\lambda^{\prime}\right)\left(\xi^{2}+1\right)}\left(e^{i x \xi}-e^{-i x \xi}\right) \underline{e} \partial_{\xi}\left[\frac{\mathcal{F}\left(\psi_{\mathrm{dis}}\right)(\xi)}{\left(\xi^{2}+1\right)^{k} \xi}\right] d \xi, \\
& \chi_{>0}(x)\left(\begin{array}{c}
\overline{\tilde{U}^{(s)}}\left(\lambda^{\prime}, \cdot\right) \\
\tilde{U}^{(s)}\left(\lambda^{\prime}, \cdot\right)
\end{array}\right) \times \frac{1}{s-\lambda^{\prime}} \int_{0}^{\infty} e^{-i\left(s-\lambda^{\prime}\right)\left(\xi^{2}+1\right)} \partial_{\xi} s(\xi) e^{i x \xi} \underline{e} \frac{\mathcal{F}\left(\psi_{\mathrm{dis}}\right)(\xi)}{\left(\xi^{2}+1\right)^{k} \xi} d \xi, \\
& \chi>0(x)\left(\begin{array}{c}
\overline{\tilde{U}^{(s)}(\lambda, \cdot)} \\
\tilde{U}^{(s)}(\lambda, \cdot)
\end{array}\right) \times \frac{1}{s-\lambda} \int_{-\infty}^{0} e^{-i(s-\lambda)\left(\xi^{2}+1\right)}\left(e^{i x \xi}-e^{-i x \xi}\right) \underline{e} \partial \underline{\xi}\left[\frac{\mathcal{F}\left(\phi_{\mathrm{dis}}\right)(\xi)}{\xi}\right] d \xi, \\
& \chi>0(x)\left(\begin{array}{c}
\overline{\tilde{U}^{(s)}(\lambda, \cdot)} \\
\tilde{U}^{(s)}(\lambda, \cdot)
\end{array}\right) \times \frac{1}{s-\lambda} \int_{0}^{\infty} e^{-i(s-\lambda)\left(\xi^{2}+1\right)} \partial_{\xi} s(\xi) e^{i x \xi} \underline{e} \frac{\mathcal{F}\left(\phi_{\mathrm{dis}}\right)(\xi)}{\xi} d \xi .
\end{aligned}
$$

$\left(\beta_{2} \beta_{2}\right)$ : This is an expression of the form

$$
\begin{aligned}
\int_{T}^{\infty} t \int_{t}^{\infty}(v-1)(s) \int_{0}^{s / 2}\left\langle\left(\begin{array}{c}
\chi>0 \\
\chi_{>0}\left|\tilde{U}^{(s)}\right|^{4}\left(\lambda^{\prime}, \cdot\right) \\
-\left.\right|^{4}\left(\lambda^{\prime}, \cdot\right)
\end{array}\right)_{\mathrm{dis}}, \beta_{2}\left(\lambda^{\prime}, \cdot\right)\right\rangle d \lambda^{\prime} \\
\\
\cdot \int_{0}^{s / 2}\left\langle\left(\begin{array}{c}
\chi>0\left|\tilde{U}^{(s)}\right|^{4}(\lambda, \cdot) \\
-\chi_{>0}\left|\tilde{U}^{(s)}\right|^{4}(\lambda, \cdot)
\end{array}\right)_{\mathrm{dis}}, \beta_{2}(\lambda, \cdot)\right\rangle d \lambda d s d t
\end{aligned}
$$

where $\beta_{2}\left(\lambda^{\prime}, \cdot\right), \beta_{2}(\lambda, \cdot)$ stand for certain terms of the second list. This type of interaction is easy to control: one bounds this by

$$
\begin{aligned}
& \lesssim \int_{T}^{\infty} t \int_{t}^{\infty} s^{-1 / 2+\delta_{1}} \int_{0}^{s / 2} \lambda^{\prime-3 / 2}\left(s-\lambda^{\prime}\right)^{-3 / 2} d \lambda^{\prime} \int_{0}^{\lambda} \lambda^{-3 / 2}(s-\lambda)^{-3 / 2} d \lambda d s d t \\
& \lesssim T^{-1 / 2+\delta_{1}} .
\end{aligned}
$$

$\left(\beta_{1} \beta_{2}\right)$ : This is an expression of the form

$$
\begin{aligned}
& \int_{T}^{\infty} t \int_{t}^{\infty}(v-1)(s) \int_{0}^{s / 2}\left\langle\left(\begin{array}{c}
\chi>0 \\
-\chi_{>0}\left|\tilde{U}^{(s)}\right|^{4}(\lambda, \cdot) \\
-\tilde{U}^{(s}(\lambda, \cdot)
\end{array}\right)_{\mathrm{dis}}, \beta_{1}(\lambda, \cdot)\right\rangle d \lambda
\end{aligned}
$$

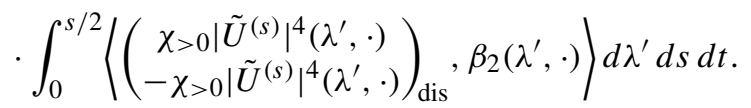


Assume for example (the other cases being treated by exact analogy) that $\beta_{2}\left(\lambda^{\prime}, \cdot\right)$ has the following form:

$\beta_{2}\left(\lambda^{\prime}, \cdot\right)=\chi>0(x)\left(\begin{array}{c}\overline{\tilde{U}^{(s)}}\left(\lambda^{\prime}, \cdot\right) \\ \tilde{U}^{(s)}\left(\lambda^{\prime}, \cdot\right)\end{array}\right) \times \frac{1}{s-\lambda^{\prime}} \int_{0}^{\infty} e^{-i\left(s-\lambda^{\prime}\right)\left(\xi^{2}+1\right)} \partial_{\xi} s(\xi) e^{i x \xi} \underline{e} \frac{\mathcal{F}\left(\phi_{\mathrm{dis}}\right)(\xi)}{\xi} d \xi$.

Note that on account of

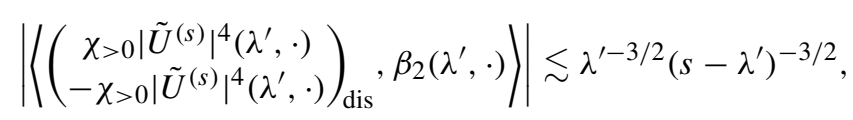

we may assume that $\lambda^{\prime}<s^{\epsilon}$ for a small $\epsilon>0$. But then on account of the pseudoconformal almost conservation we may apply a localizer $\phi_{<s^{2 \epsilon}}(x)$ to the quadrilinear term: indeed, we have

$$
\begin{aligned}
& \left|\left\langle\left(\begin{array}{c}
\chi>0 \phi_{\geq s^{2 \epsilon}}(x)\left|\tilde{U}^{(s)}\right|^{4}\left(\lambda^{\prime}, \cdot\right) \\
-\chi>0 \phi_{\geq s^{2 \epsilon}}(x)\left|\tilde{U}^{(s)}\right|^{4}\left(\lambda^{\prime}, \cdot\right)
\end{array}\right)_{\mathrm{dis}}, \beta_{2}\left(\lambda^{\prime}, \cdot\right)\right\rangle\right| \\
& \lesssim\left\|\frac{1}{|x|} \phi_{\geq s^{2 \epsilon}}(|x|)\left[\left(x+2 i p \lambda^{\prime}\right) U-2 i p \lambda^{\prime} U\right]\right\|_{L_{x}^{2}}\|U(\lambda, \cdot)\|_{L_{x}^{2}}\left\|U\left(\lambda^{\prime}, \cdot\right)\right\|_{L_{x}^{\infty}}^{3}\left(s-\lambda^{\prime}\right)^{-3 / 2} \\
& \lesssim s^{-\epsilon+\delta_{2}} \lambda^{\prime-3 / 2}\left(s-\lambda^{\prime}\right)^{-3 / 2},
\end{aligned}
$$

which leads to an acceptable contribution above. Finally, we may reduce the frequency $\xi$ in the relation defining $\beta_{2}\left(\lambda^{\prime}, \cdot\right)$ above to absolute size $<s^{-\epsilon}$ by inclusion of a suitable smooth cutoff $\phi_{<s^{-\epsilon}}(\xi)$. This is since including a smooth cutoff $\phi_{\geq s^{-\epsilon}}(\xi)$ for suitably small $\epsilon$ results in an expression which can be integrated by parts in $\xi$, resulting in losses of at most $\max \left\{|x|, s^{\epsilon}\right\} s^{\epsilon}$ for each integration while resulting in a gain of $s-\lambda^{\prime}$. Choosing $\epsilon$ small enough results in arbitrary gains in $s$. Next, we consider $\beta_{1}(\lambda, \cdot)$. Using the same argument as in case (BC), we reduce the frequency to size $>\lambda^{-\epsilon_{1}}$. But then we have again achieved frequency separation and can integrate by parts in $s$. The case $\left(\beta_{2} \beta_{1}\right)$ is simpler, as one gains $\lambda^{-1 / 2}$ which suffices (since $\lambda>\lambda^{\prime}$ ).

$\left(\beta_{1} \beta_{1}\right)$ : This is an expression of the form

$$
\begin{array}{r}
\int_{T}^{\infty} t \int_{t}^{\infty}(v-1)(s) \int_{0}^{s / 2}\left\langle\left(\begin{array}{c}
\chi>0\left|\tilde{U}^{(s)}\right|^{4}\left(\lambda^{\prime}, \cdot\right) \\
-\chi>0\left|\tilde{U}^{(s)}\right|^{4}\left(\lambda^{\prime}, \cdot\right)
\end{array}\right)_{\mathrm{dis}}, \beta_{1}\left(\lambda^{\prime}, \cdot\right)\right\rangle d \lambda^{\prime} \\
\cdot \int_{0}^{s / 2}\left\langle\left(\begin{array}{c}
\chi>0\left|\tilde{U}^{(s)}\right|^{4}(\lambda, \cdot) \\
-\chi_{>0}\left|\tilde{U}^{(s)}\right|^{4}(\lambda, \cdot)
\end{array}\right)_{\mathrm{dis}}, \beta_{1}(\lambda, \cdot)\right\rangle d \lambda .
\end{array}
$$

Keep in mind that we assume $\lambda>\lambda^{\prime}$. Use the distorted Plancherel's Theorem 2.3 to rewrite this as

$$
\begin{aligned}
\sum_{ \pm, \pm} \int_{T}^{\infty} t \int_{t}^{\infty}(v-1)(s) \int_{0}^{s / 2} \int_{-\infty}^{\infty} \mathcal{F}_{ \pm}\left(\begin{array}{c}
\chi>0\left|\tilde{U}^{(s)}\right|^{4}\left(\lambda^{\prime}, \cdot\right) \\
-\chi>0\left|\tilde{U}^{(s)}\right|^{4}\left(\lambda^{\prime}, \cdot\right)
\end{array}\right)(\xi) \overline{\tilde{\mathcal{F}}_{ \pm}\left[\beta_{1}\left(\lambda^{\prime}, \cdot\right)\right]} d \xi d \lambda^{\prime} \\
\cdot \int_{0}^{s / 2} \int_{-\infty}^{\infty} \mathcal{F}_{ \pm}\left(\begin{array}{c}
\chi_{>0}\left|\tilde{U}^{(s)}\right|^{4}(\lambda, \cdot) \\
-\chi_{>0}\left|\tilde{U}^{(s)}\right|^{4}(\lambda, \cdot)
\end{array}\right)\left(\xi^{\prime}\right) \overline{\tilde{\mathcal{F}}_{ \pm}\left[\beta_{1}(\lambda, \cdot)\right]\left(\xi^{\prime}\right)} d \xi^{\prime} d \lambda d s d t
\end{aligned}
$$


We may and shall restrict to the + case and omit the subscript, and restrict both the $\xi$ and $\xi^{\prime}$ integrals to the range $(-\infty, 0]$, the other case being similar but simpler. We then need to decompose each of the Fourier transforms $\mathcal{F}(\ldots)$ etc. into various constituents, i.e., write

$$
\begin{aligned}
& \mathcal{F}\left(\begin{array}{c}
\chi_{>0}\left|\tilde{U}^{(s)}\right|^{4}\left(\lambda^{\prime}, \cdot\right) \\
-\chi>0\left|\tilde{U}^{(s)}\right|^{4}\left(\lambda^{\prime}, \cdot\right)
\end{array}\right)(\xi)=\left\langle\left(\begin{array}{c}
\chi>0\left|\tilde{U}^{(s)}\right|^{4}\left(\lambda^{\prime}, \cdot\right) \\
-\chi_{>0}\left|\tilde{U}^{(s)}\right|^{4}\left(\lambda^{\prime}, \cdot\right)
\end{array}\right), \phi(x, \xi)\right\rangle \\
& +\left\langle\left(\begin{array}{c}
\chi_{>0}\left|\tilde{U}^{(s)}\right|^{4}\left(\lambda^{\prime}, \cdot\right) \\
-\chi>0\left|\tilde{U}^{(s)}\right|^{4}\left(\lambda^{\prime}, \cdot\right)
\end{array}\right),(1+r(-\xi)) \underline{e} e^{-i x \xi}\right\rangle+\left\langle\left(\begin{array}{c}
\chi_{>0}\left|\tilde{U}^{(s)}\right|^{4}\left(\lambda^{\prime}, \cdot\right) \\
-\chi>0\left|\tilde{U}^{(s)}\right|^{4}\left(\lambda^{\prime}, \cdot\right)
\end{array}\right),\left(e^{i x \xi}-e^{-i x \xi}\right) \underline{e}\right\rangle .
\end{aligned}
$$

We shall consider the contribution from the first and third terms, the second being treated similarly to the third. Moreover, performing the same decomposition for $\tilde{\mathcal{F}}\left[\beta_{1}\left(\lambda^{\prime}, \cdot\right)\right]$ as well as $\mathcal{F}\left(\begin{array}{c}\chi_{>0}\left|\tilde{U}^{(s)}\right|^{4}(\lambda, \cdot) \\ -\chi>0 \mid \\ \left.\tilde{U}^{(s)}\right|^{4}(\lambda, \cdot)\end{array}\right)\left(\xi^{\prime}\right)$, it is easy to see that we may restrict to the contribution from the third term, as the others are simpler. We commence with the following expression:

$$
\begin{aligned}
& \sum_{ \pm, \pm} \int_{T}^{\infty} t \int_{t}^{\infty}(v-1)(s)
\end{aligned}
$$

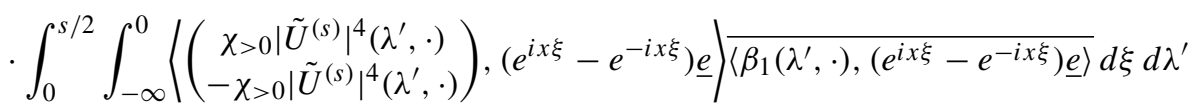

$$
\begin{aligned}
& \cdot \int_{0}^{s / 2} \int_{-\infty}^{0}\left\langle\left(\begin{array}{c}
\chi>0\left|\tilde{U}^{(s)}\right|^{4}(\lambda, \cdot) \\
-\chi_{>0}\left|\tilde{U}^{(s)}\right|^{4}(\lambda, \cdot)
\end{array}\right),\left(e^{i x \xi^{\prime}}-e^{-i x \xi^{\prime}}\right) \underline{e}\right| \overline{\left\langle\beta_{1}(\lambda, \cdot),\left(e^{i x \xi^{\prime}}-e^{-i x \xi^{\prime}}\right) \underline{e}\right\rangle} d \xi^{\prime} d \lambda d s d t .
\end{aligned}
$$

If we recapitulate the proof of SLDE for both bracket factors, we see that we may reduce to estimating

$$
\begin{aligned}
& \sum_{ \pm, \pm} \int_{T}^{\infty} t \int_{t}^{\infty}(v-1)(s) \\
& \cdot \int_{0}^{s / 2} \int_{-\infty}^{0}\left\langle\left(\begin{array}{c}
P_{<a^{\prime}}\left[\chi_{>0}\left|\tilde{U}^{(s)}\right|^{2}\right] P_{<a^{\prime}}\left[\left|\tilde{U}^{(s)}\right|^{2}\right]\left(\lambda^{\prime}, \cdot\right) \\
-P_{<a^{\prime}}\left[\chi_{>0}\left|\tilde{U}^{(s)}\right|^{2}\right] P_{<a^{\prime}}\left[\left|\tilde{U}^{(s)}\right|^{2}\right]\left(\lambda^{\prime}, \cdot\right)
\end{array}\right),\left(e^{i x \xi}-e^{-i x \xi}\right) \underline{e}\right\rangle \\
& \cdot \overline{\left\langle\beta_{1}\left(\lambda^{\prime}, \cdot\right),\left(e^{i x \xi}-e^{-i x \xi}\right) \underline{e}\right\rangle} d \xi d \lambda^{\prime} \\
& \cdot \int_{0}^{s / 2} \int_{-\infty}^{0}\left\langle\left(\begin{array}{c}
P_{<a}\left[\chi_{>0}\left|\tilde{U}^{(s)}\right|^{2}\right] P_{<a}\left[\left|\tilde{U}^{(s)}\right|^{2}\right](\lambda, \cdot) \\
-P_{<a}\left[\chi_{>0}\left|\tilde{U}^{(s)}\right|^{2}\right] P_{<a}\left[\left|\tilde{U}^{(s)}\right|^{2}\right](\lambda, \cdot)
\end{array}\right),\left(e^{i x \xi^{\prime}}-e^{-i x \xi^{\prime}}\right) \underline{e}\right\rangle \\
& \cdot \overline{\left\langle\beta_{1}(\lambda, \cdot),\left(e^{i x \xi^{\prime}}-e^{-i x \xi^{\prime}}\right) \underline{e}\right\rangle} d \xi^{\prime} d \lambda d s d t,
\end{aligned}
$$

where $a=\lambda^{-3 / 4+\epsilon_{2}}, a^{\prime}=\lambda^{\prime-3 / 4} \lambda^{\epsilon_{2}}, \epsilon_{2}=\epsilon_{2}\left(\delta_{2}\right)$. Recalling the product representation of $\beta_{1}\left(\lambda^{\prime}, \cdot\right)$ as in 5.50 , we first reduce the frequency of the right hand integral factor of both $\beta_{1}(\lambda, \cdot), \beta_{1}\left(\lambda^{\prime}, \cdot\right)$ to size $>\lambda^{-\epsilon}$. This is achieved as in case (BC). For technical reasons we shall effect this by means of a sharp cutoff $\chi_{>\lambda^{-\epsilon}}(\xi)$ etc. Thus for example

\footnotetext{
66 The same argument applies to all terms of the list $\beta_{1}$.
} 
we shall put

$$
\beta_{1}(\lambda, x)=\left(\begin{array}{c}
\overline{\tilde{U}^{(s)}}(\lambda, \cdot) \\
x \tilde{U}^{(s)}(\lambda, \cdot)
\end{array}\right) \times \chi>0(x) \frac{e^{ \pm i(s-\lambda)}}{(s-\lambda)^{3 / 2}} \int_{-\infty}^{\infty} e^{\frac{ \pm(x-y)^{2}}{i(s-\lambda)}} g(y) d y,
$$

where

$$
g(y)=\phi_{<\lambda^{1 / 10}}(y) \int_{-\infty}^{0} \chi_{>\lambda^{-\epsilon}}(\xi)\left(e^{i y \xi}+e^{-i y \xi}\right) \underline{e}\left[\frac{\mathcal{F}\left(\phi_{\mathrm{dis}}\right)(\xi)}{\xi}\right] d \xi,
$$

where $\chi_{>\lambda-\epsilon}(\xi)$ is a Heavyside function. Of course we have

$$
\frac{1}{\sqrt{s-\lambda}} \int_{-\infty}^{\infty} e^{\frac{ \pm(x-y)^{2}}{i(s-\lambda)}} g(y) d y=\int_{-\infty}^{\infty} e^{\mp i(s-\lambda) \xi^{2}} e^{i x \xi} \hat{g}(\xi) d \xi .
$$

Similar observations apply to $\beta_{1}\left(\lambda^{\prime}, \cdot\right)$, for example

$$
\beta_{1}\left(\lambda^{\prime}, x\right)=\left(\begin{array}{c}
\overline{\tilde{U}^{(s)}}\left(\lambda^{\prime}, \cdot\right) \\
x \tilde{U}^{(s)}\left(\lambda^{\prime}, \cdot\right)
\end{array}\right) \times \chi>0(x) \frac{e^{\mp i\left(s-\lambda^{\prime}\right)}}{\left(s-\lambda^{\prime}\right)^{3 / 2}} \int_{-\infty}^{\infty} e^{ \pm \frac{(x-y)^{2}}{i(s-\lambda)}} \tilde{g}(y) d y,
$$

where

$$
\tilde{g}(y)=\phi_{<\lambda^{1 / 10}}(y) \int_{0}^{\infty} \chi_{>\lambda^{-\epsilon}}\left(\xi^{\prime}\right) s\left(\xi^{\prime}\right) e^{i y \xi^{\prime}} \frac{\mathcal{F}\left(\phi_{\mathrm{dis}}\right)\left(\xi^{\prime}\right)}{\left(\xi^{\prime 2}+1\right)^{k} \xi^{\prime}} d \xi^{\prime} .
$$

We now further specialize the frequency support of $g(y), \tilde{g}(y)$, by including cutoffs $\chi_{I_{i}}(\xi), \chi_{I_{j}}\left(\xi^{\prime}\right)$ corresponding to intervals $I_{i, j}$ of length $\lambda^{-\epsilon}$, i.e., introduce

$$
\begin{aligned}
& g_{i}(y)=\phi_{<\lambda^{1 / 10}}(y) \int_{-\infty}^{0} \chi_{>\lambda^{-\epsilon}}(\xi) \chi_{I_{i}}(\xi)\left(e^{i y \xi}+e^{-i y \xi}\right) \underline{e}\left[\frac{\mathcal{F}\left(\phi_{\mathrm{dis}}\right)(\xi)}{\xi}\right] d \xi, \\
& \tilde{g}_{j}(y)=\phi_{<\lambda^{1 / 10}}(y) \int_{0}^{\infty} \chi_{>\lambda-\epsilon}\left(\xi^{\prime}\right) s\left(\xi^{\prime}\right) e^{i y \xi^{\prime}} \chi_{j}\left(\xi^{\prime}\right) \underline{e} \frac{\mathcal{F}\left(\phi_{\mathrm{dis}}\right)\left(\xi^{\prime}\right)}{\left(\xi^{\prime 2}+1\right)^{k} \xi^{\prime}} d \xi^{\prime} .
\end{aligned}
$$

Clearly if $|i-j| \gg 1$ these functions have separated Fourier supports (of distance $\gtrsim \lambda^{-\epsilon}$ ) up to errors of order $\lambda^{-N}$, hence negligible. Now introduce $\beta_{1, i}\left(\lambda^{\prime}, \cdot\right), \beta_{1, j}(\lambda, \cdot)$ exactly as above with $g(y), \tilde{g}(y)$ replaced by $g_{i}(y), \tilde{g}_{j}(y)$. It is easy to see that we can restrict both $|\xi|,\left|\xi^{\prime}\right|$ to size $<\lambda^{\epsilon_{3}}$ for $\epsilon_{3}=\epsilon_{3}\left(\delta_{2}\right)$, since otherwise one gains enough to overcome any losses in the proof of SLDE.

Case $1:|i-j| \gg 1$. Here we exploit integration by parts in $s$. Write

$$
\beta_{1, i}(\lambda, x)=\left(\begin{array}{c}
\overline{\tilde{U}^{(s)}}(\lambda, \cdot) \\
x \tilde{U}^{(s)}(\lambda, \cdot)
\end{array}\right) \times \chi_{>0}(x) \frac{e^{ \pm i(s-\lambda)}}{(s-\lambda)^{3 / 2}} \int_{-\infty}^{\infty} e^{\mp i(s-\lambda) \xi^{2}} e^{i x \xi} \hat{g}_{i}(\xi) d \xi
$$

and similarly for $\beta_{1, j}\left(\lambda^{\prime}, \cdot\right)$. Then rewrite 


$$
\begin{aligned}
& \sum_{ \pm, \pm} \int_{T}^{\infty} t \int_{t}^{\infty}(v-1)(s) \\
& \int_{0}^{s / 2} \int_{-\infty}^{0}\left\langle\left(\begin{array}{c}
P_{<a^{\prime}}\left[\chi_{>0}\left|\tilde{U}^{(s)}\right|^{2}\right] P_{<a^{\prime}}\left[\left|\tilde{U}^{(s)}\right|^{2}\right]\left(\lambda^{\prime}, \cdot\right) \\
-P_{<a^{\prime}}\left[\chi_{>0}\left|\tilde{U}^{(s)}\right|^{2}\right] P_{<a^{\prime}}\left[\left|\tilde{U}^{(s)}\right|^{2}\right]\left(\lambda^{\prime}, \cdot\right)
\end{array}\right),\left(e^{i x \xi}-e^{-i x \xi}\right) \underline{e}\right\rangle \\
& \cdot \overline{\left\langle\beta_{1, j}\left(\lambda^{\prime}, \cdot\right),\left(e^{i x \xi}-e^{-i x \xi}\right) \underline{e}\right\rangle} d \xi d \lambda^{\prime} \\
& \cdot \int_{0}^{s / 2} \int_{-\infty}^{0}\left\langle\left(\begin{array}{c}
P_{<a}\left[\chi_{>0}\left|\tilde{U}^{(s)}\right|^{2}\right] P_{<a}\left[\left|\tilde{U}^{(s)}\right|^{2}\right](\lambda, \cdot) \\
-P_{<a}\left[\chi_{>0}\left|\tilde{U}^{(s)}\right|^{2}\right] P_{<a}\left[\left|\tilde{U}^{(s)}\right|^{2}\right](\lambda, \cdot)
\end{array}\right),\left(e^{i x \xi^{\prime}}-e^{-i x \xi^{\prime}}\right) \underline{e}\right\rangle \\
& \cdot \frac{\left\langle\beta_{1, i}(\lambda, \cdot),\left(e^{i x \xi^{\prime}}-e^{-i x \xi^{\prime}}\right) \underline{e}\right\rangle}{d \xi^{\prime}} d \lambda d s d t
\end{aligned}
$$

as

$\int_{T}^{\infty} t \int_{t}^{\infty}(v-1)(s) \int_{-\infty}^{0} \int_{-\infty}^{0} \int_{0}^{\infty} \int_{0}^{\infty} e^{i s\left( \pm \xi^{2} \pm \xi^{2}\right)} \Psi_{i j}\left(s, \lambda, \lambda^{\prime}, \xi, \xi^{\prime}\right) d \xi d \xi^{\prime} d \lambda d \lambda^{\prime} d s d t$

switch the order of integration, integrate by parts in $s$, decouple the variables $\xi$, $\xi^{\prime}$ by means of discrete Fourier transform and proceed as in the proof of SLDE. Choosing $\epsilon>0$ small enough results in a gain in $\lambda$, even upon summing over $i, j$. This concludes Case 1.

Case 2: $i=j+O(1)$. First write

$$
\beta_{1, i}=2 \sqrt{-1} \beta_{1, i}^{a}+\beta_{1, i}^{b}
$$

where

$$
\begin{aligned}
& \beta_{1, i}^{a}=\lambda\left(\begin{array}{c}
\overline{\partial_{x} \tilde{U}^{(s)}}(\lambda, \cdot) \\
-\partial_{x} \tilde{U}^{(s)}(\lambda, \cdot)
\end{array}\right) \times \chi>0(x) \frac{e^{ \pm i(s-\lambda)}}{(s-\lambda)^{3 / 2}} \int_{-\infty}^{\infty} e^{\frac{ \pm(x-y)^{2}}{i(s-\lambda)}} g_{i}(y) d y, \\
& \beta_{1, i}^{b}=\left(\begin{array}{l}
(x+2 \lambda p) \tilde{U}^{(s)}(\lambda, \cdot) \\
(x+2 \lambda p) \tilde{U}^{(s)}(\lambda, \cdot)
\end{array}\right) \times \chi_{>0}(x) \frac{e^{ \pm i(s-\lambda)}}{(s-\lambda)^{3 / 2}} \int_{-\infty}^{\infty} e^{\frac{ \pm(x-y)^{2}}{i(s-\lambda)}} g_{i}(y) d y .
\end{aligned}
$$

From the proof of SLDE, recall that we use integration by parts to write

$$
\begin{aligned}
&\left\langle\beta_{1, i}^{(a)}(\lambda, \cdot),\left(e^{i x \xi}-e^{-i x \xi}\right) \underline{e}\right\rangle \\
&=i \lambda \xi \int_{0}^{\infty}\left(e^{i x \xi}+e^{-i x \xi}\right)\left\langle\underline{e}, \int_{x}^{\infty} \chi>0(y)\left(\begin{array}{c}
\overline{\partial_{y} \tilde{U}^{(s)}}(\lambda, y) \\
-\partial_{y} \tilde{U}^{(s)}(\lambda, y)
\end{array}\right)\right. \\
&\left.\cdot \frac{e^{ \pm i(s-\lambda)}}{(s-\lambda)^{3 / 2}} \int_{-\infty}^{\infty} e^{\frac{ \pm(y-z)^{2}}{i(s-\lambda)}} g_{i}(z) d z d y\right\rangle d x .
\end{aligned}
$$

Still following the proof of SLDE in the low-low case, we then use the free parametrix to write schematically

$$
\partial_{y} \overline{\tilde{U}^{(s)}}(\lambda, y)=\int_{0}^{\lambda} \frac{1}{\sqrt{\lambda-\mu}} \int_{-\infty}^{\infty} e^{\frac{\left(y-z^{\prime}\right)^{2}}{i(\lambda-\mu)}} \partial_{z^{\prime}}\left[|U|^{4} U\left(\mu, z^{\prime}\right)+V U\left(\mu, z^{\prime}\right)\right] d z^{\prime} d \mu .
$$


We then break this into the contributions from $\chi_{><\lambda^{1 / 2+\epsilon_{3}}}(\mu) \chi_{><(\lambda-\mu)^{1 / 2+\epsilon_{3}}}\left(\left|z^{\prime}\right|\right)|U|^{4}$

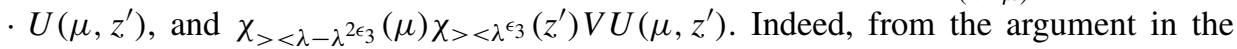
proof of SLDE, it follows that if one $>$ sign is chosen in these cutoffs, the corresponding contribution leads to a small extra gain in $\lambda$, which then suffices to close, provided $\epsilon_{3}=\epsilon_{3}\left(\delta_{2}\right)$. Thus we now choose everywhere the $<$ sign, and substitute this into (5.55). Collecting the exponentials, we encounter the following phase function, just as in the proof of SLDE:

$$
\begin{aligned}
& e^{\frac{y^{2}}{i}\left[\frac{1}{\lambda-\mu}+\frac{1}{s-\lambda}\right]-\frac{2 x}{i}\left[\frac{z^{\prime}}{\lambda-\mu}+\frac{z}{s-\lambda}\right]} e^{z^{2} / i(\lambda-\mu)+\frac{z^{2}}{i(s-\lambda)}} \\
& =e^{-i\left(y \sqrt{\frac{1}{\lambda-\mu}+\frac{1}{s-\lambda}}-\left[\frac{z^{\prime}}{\lambda-\mu}+\frac{z}{s-\lambda}\right]\left[\sqrt{\frac{1}{\lambda-\mu}+\frac{1}{s-\lambda}}\right]^{-1}\right)^{2}} e^{z^{\prime 2} / i(\lambda-\mu)+\frac{z^{2}}{i(s-\lambda)}+i\left[\frac{z^{\prime}}{\lambda-\mu}+\frac{z}{s-\lambda}\right]^{2}\left[\frac{1}{\lambda-\mu}+\frac{1}{s-\lambda}\right]^{-1}} \\
& =: e^{-i\left(y \sqrt{\frac{1}{\lambda-\mu}+\frac{1}{s-\lambda}}-y_{1}\right)^{2}} e^{i y_{2}},
\end{aligned}
$$

where we have

$$
\left|y_{1,2}\right|=\left|y_{1,2}\left(z, z^{\prime}, \lambda, \mu, s\right)\right| \lesssim \lambda^{\epsilon_{3}}
$$

on the support of the integrand in 5.55). Thus plugging this into 5.55 and omitting the integration in $\mu^{\prime}, \mu$ for now, we obtain the expression

$$
\begin{aligned}
& \frac{1}{\sqrt{(s-\lambda)(\lambda-\mu)}} \int_{-\infty}^{\infty} \int_{-\infty}^{\infty} \int_{0}^{\infty}\left(e^{i x \xi}+e^{-i x \xi}\right) \\
& \cdot \int_{x}^{\infty} e^{-i\left(y \sqrt{\frac{1}{\lambda-\mu}+\frac{1}{s-\lambda}}-y_{1}\right)^{2}} e^{i y_{2}} g_{i}(z) g_{1}\left(\mu, z^{\prime}\right) d y d x d z d z^{\prime} \\
& =\frac{\left[\frac{1}{s-\lambda}+\frac{1}{\lambda-\mu}\right]^{-1}}{\sqrt{(s-\lambda)(\lambda-\mu)}} \int_{-\infty}^{\infty} \int_{-\infty}^{\infty} e^{i y_{2}} \int_{-y_{1}}^{\infty}\left[e^{i \xi\left[\tilde{x}+y_{1}\right]\left[\sqrt{\frac{1}{s-\lambda}+\frac{1}{\lambda-\mu}}\right]^{-1}}+e^{-i \xi\left[\tilde{x}+y_{1}\right]\left[\sqrt{\frac{1}{s-\lambda}+\frac{1}{\lambda-\mu}}\right]^{-1}}\right] \\
& \cdot \int_{\tilde{x}}^{\infty} e^{i \rho^{2}} d \rho d \tilde{x} \phi_{<\lambda} 1 / 10(z) \int_{-\infty}^{0} \chi_{>\lambda}-\epsilon(\eta) \chi_{I_{i}}(\eta)\left(e^{i z \eta}+e^{-i z \eta}\right) \underline{e}\left[\frac{\mathcal{F}\left(\phi_{\mathrm{dis}}\right)(\eta)}{\eta}\right] d \eta g_{1}\left(z^{\prime}, \mu\right) d z d z^{\prime} .
\end{aligned}
$$

Now assume $I_{i}=\left[a_{i}, b_{i}\right]$ where $\min \left\{\left|a_{i}\right|,\left|b_{i}\right|\right\} \geq \lambda^{-\epsilon}$. Then integrate by parts in

$$
\begin{array}{r}
\int_{-\infty}^{0} \chi_{>\lambda^{-\epsilon}}(\eta) \chi_{I_{i}}(\eta)\left(e^{i z \eta}+e^{-i z \eta}\right) \underline{e}\left[\frac{\mathcal{F}\left(\phi_{\mathrm{dis}}\right)(\eta)}{\eta}\right] d \eta=\frac{e^{i z a_{i}} \frac{\mathcal{F}\left(\phi_{\mathrm{dis}}\right)\left(a_{i}\right)}{a_{i}}-e^{i z b_{i}} \frac{\mathcal{F}\left(\phi_{\mathrm{dis}}\right)\left(b_{i}\right)}{b_{i}}}{i z} \\
-\frac{1}{i z} \int_{-\infty}^{0} \chi_{>\lambda^{-\epsilon}}(\eta) \chi_{I_{i}}(\eta)\left(e^{i z \eta}-e^{-i z \eta}\right) \underline{e} \partial_{\eta}\left[\frac{\mathcal{F}\left(\phi_{\mathrm{dis}}\right)(\eta)}{\eta}\right] d \eta
\end{array}
$$

Observe that

$$
\left\|\phi_{<\lambda^{1 / 10}}(z) \frac{1}{i z} \int_{-\infty}^{0} \chi_{>\lambda^{-\epsilon}}(\eta) \chi_{I_{i}}(\eta)\left(e^{i z \eta}-e^{-i z \eta}\right) \underline{e} \partial_{\eta}\left[\frac{\mathcal{F}\left(\phi_{\text {dis }}\right)(\eta)}{\eta}\right] d \eta\right\|_{L_{z}^{1}} \lesssim(\log \lambda) \lambda^{-\epsilon}
$$


whence this expression has a negligible contribution upon continuing the proof of SLDE and choosing $\epsilon_{3} \ll \epsilon$. We further observe that the restriction $|\xi|<\lambda^{-3 / 4+\epsilon}$ as well as the restrictions on $|z|,\left|z^{\prime}\right|, \lambda$ and $\mu$ specified further above imply that

$$
\begin{gathered}
\left|\partial_{z}\left[e^{i y_{2}} \int_{-y_{1}}^{\infty}\left[e^{i \xi\left[\tilde{x}+y_{1}\right]\left[\sqrt{\frac{1}{s-\lambda}+\frac{1}{\lambda-\mu}}\right]^{-1}}+e^{-i \xi\left[\tilde{x}+y_{1}\right]\left[\sqrt{\frac{1}{s-\lambda}+\frac{1}{\lambda-\mu}}\right]^{-1}}\right] \int_{\tilde{x}}^{\infty} e^{i \rho^{2}} d \rho d \tilde{x}\right]\right| \\
\left|\left[e^{i y_{2}} \int_{-y_{1}}^{\infty}\left[e^{\left.i \xi \tilde{x}+y_{1}\right]\left[\sqrt{\frac{1}{s-\lambda}+\frac{1}{\lambda-\mu}}\right]^{-1}}+e^{-i \xi\left[\tilde{x}+y_{1}\right]\left[\sqrt{\frac{1}{s-\lambda}+\frac{1}{\lambda-\mu}}\right]^{-1}}\right] \int_{\tilde{x}}^{\infty} e^{i \rho^{2}} d \rho d \tilde{x}\right]\right| \lesssim \lambda^{\epsilon_{3}} .
\end{gathered}
$$

Thus to all intents and purposes we can replace the latter function by a constant as far as its dependence of $z$ on $\left[-\lambda^{1 / 10}, \lambda^{1 / 10}\right]$ is concerned. But then one calculates

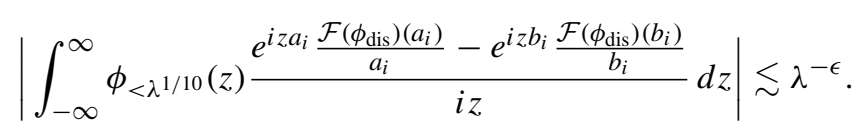

Putting everything after (5.55) together, we see that

$$
\left|\left\langle\beta_{1, i}^{(a)}(\lambda, \cdot),\left(e^{i x \xi}-e^{-i x \xi}\right) \underline{e}\right\rangle\right| \lesssim \lambda \lambda^{-3 / 4+\epsilon_{2}} \lambda^{1 / 2}(\log \lambda) \lambda^{-\epsilon}(s-\lambda)^{-3 / 2},
$$

which then yields

$$
\begin{aligned}
& \mid \int_{-\infty}^{0}\left\langle\left(\begin{array}{c}
P_{<a}\left[\chi_{>0}\left|\tilde{U}^{(s)}\right|^{2}\right] P_{<a}\left[\left|\tilde{U}^{(s)}\right|^{2}\right](\lambda, \cdot) \\
-P_{<a}\left[\chi_{>0}\left|\tilde{U}^{(s)}\right|^{2}\right] P_{<a}\left[\left|\tilde{U}^{(s)}\right|^{2}\right](\lambda, \cdot)
\end{array}\right),\left(e^{i x \xi^{\prime}}-e^{-i x \xi^{\prime}}\right) \underline{e}\right\rangle \\
& \cdot \frac{\cdot\left\langle\beta_{1, i}^{(a)}(\lambda, \cdot),\left(e^{i x \xi^{\prime}}-e^{-i x \xi^{\prime}}\right) \underline{e}\right\rangle}{\xi^{\prime}} \mid \\
& \quad \lesssim \lambda^{-1} \lambda^{-3 / 4+\epsilon_{2}} \lambda \lambda^{-3 / 4+\epsilon_{2}} \lambda^{1 / 2}(\log \lambda) \lambda^{-\epsilon}(s-\lambda)^{-3 / 2} \lesssim(\log \lambda) \lambda^{-1+2 \epsilon_{2}-\epsilon}(s-\lambda)^{-3 / 2} .
\end{aligned}
$$

We shall choose $0<\epsilon_{2} \ll \epsilon$. Analogously to $[5.55$, we also need to estimate the contribution of

$$
\left\langle\beta_{1, i}^{(b)}(\lambda, \cdot),\left(e^{i x \xi}-e^{-i x \xi}\right) \underline{e}\right\rangle .
$$

This, however, is more elementary, as we can estimate

$$
\begin{aligned}
& \|\left\langle\left(e^{i x \xi}-e^{-i x \xi}\right) \underline{e},\left(\begin{array}{l}
(x+2 \lambda p) \tilde{U}^{(s)}(\lambda, \cdot) \\
(x+2 \lambda p) \tilde{U}^{(s)}(\lambda, \cdot)
\end{array}\right)\right. \\
&\left.\quad \times \quad \chi>0(x) \frac{e^{ \pm i(s-\lambda)}}{(s-\lambda)^{3 / 2}} \int_{-\infty}^{\infty} e^{\frac{ \pm(x-y)^{2}}{i(s-\lambda)}} g_{i}(y) d y\right) \|_{L_{\xi}^{2}} \\
& \quad \lesssim\left\|(x+2 \lambda p) \tilde{U}^{(s)}(\lambda, \cdot)\right\|_{L_{x}^{2}}(s-\lambda)^{-3 / 2} \lesssim(s-\lambda)^{-3 / 2},
\end{aligned}
$$

whence we get

$$
\begin{aligned}
& \mid \int_{-\infty}^{0}\left\langle\left(\begin{array}{c}
P_{<a}\left[\chi>0\left|\tilde{U}^{(s)}\right|^{2}\right] P_{<a}\left[\left|\tilde{U}^{(s)}\right|^{2}\right]\left(\lambda^{\prime}, \cdot\right) \\
-P_{<a}\left[\chi>0\left|\tilde{U}^{(s)}\right|^{2}\right] P_{<a}\left[\left|\tilde{U}^{(s)}\right|^{2}\right]\left(\lambda^{\prime}, \cdot\right)
\end{array}\right),\left(e^{i x \xi^{\prime}}-e^{-i x \xi^{\prime}}\right) \underline{e}\right\rangle \\
& \cdot \overline{\left\langle\beta_{1, i}^{(a)}(\lambda, \cdot),\left(e^{i x \xi^{\prime}}-e^{-i x \xi^{\prime}}\right) \underline{e}\right\rangle} d \xi^{\prime} \mid \lesssim \lambda^{-3 / 2}(s-\lambda)^{-3 / 2},
\end{aligned}
$$


which then leads to an acceptable contribution. Now of course we eventually need to estimate the expression (5.54) under our current assumption $i=j+O(1)$, and then sum over $i$. One can replicate the preceding arguments for $\beta_{1, j}\left(\lambda^{\prime}, \cdot\right)$ as long as $\lambda^{\prime}>\lambda^{10 \epsilon}$, say. But we can exclude the opposite case, since if $\lambda^{\prime} \leq \lambda^{10 \epsilon}$, we can restrict $U\left(\lambda^{\prime}, x\right)$ to the range $|x|<\lambda^{20 \epsilon}$, say, using pseudo-conformal almost conservation, and then we can restrict $e^{-i\left(s-\lambda^{\prime}\right) \mathcal{H}^{*}}\left(\mathcal{H}^{*}\right)^{-k} \phi_{\text {dis }}$ to frequency $<s^{-10 \epsilon}$, say, provided $\epsilon$ is small enough. Thus we can reduce this situation to the separated frequency case. Finally, if $\lambda^{\prime}>\lambda^{10 \epsilon}$, we get

$$
\begin{aligned}
& \mid \int_{-\infty}^{0}\left\langle\left(\begin{array}{c}
P_{<a}\left[\chi_{>0}\left|\tilde{U}^{(s)}\right|^{2}\right] P_{<a}\left[\left|\tilde{U}^{(s)}\right|^{2}\right](\lambda, \cdot) \\
-P_{<a}\left[\chi_{>0}\left|\tilde{U}^{(s)}\right|^{2}\right] P_{<a}\left[\left|\tilde{U}^{(s)}\right|^{2}\right](\lambda, \cdot)
\end{array}\right),\left(e^{i x \xi^{\prime}}-e^{-i x \xi^{\prime}}\right) \underline{e}\right\rangle \\
& \cdot \overline{\left\langle\beta_{1, i}^{(a)}(\lambda, \cdot),\left(e^{i x \xi^{\prime}}-e^{-i x \xi^{\prime}}\right) \underline{e}\right\rangle} d \xi^{\prime} \mid \\
& \cdot \mid \int_{-\infty}^{0}\left\langle\left(\begin{array}{c}
P_{<a}\left[\chi_{>0}\left|\tilde{U}^{(s)}\right|^{2}\right] P_{<a}\left[\left|\tilde{U}^{(s)}\right|^{2}\right]\left(\lambda^{\prime}, \cdot\right) \\
-P_{<a}\left[\chi_{>0}\left|\tilde{U}^{(s)}\right|^{2}\right] P_{<a}\left[\left|\tilde{U}^{(s)}\right|^{2}\right]\left(\lambda^{\prime}, \cdot\right)
\end{array}\right),\left(e^{i x \xi^{\prime}}-e^{-i x \xi^{\prime}}\right) \underline{e}\right\rangle \\
& \cdot \overline{\left\langle\beta_{1, j}^{(b)}(\lambda, \cdot),\left(e^{i x \xi^{\prime}}-e^{-i x \xi^{\prime}}\right) \underline{e}\right\rangle} d \xi^{\prime} \\
& \lesssim \lambda^{-1} \lambda^{-3 / 4+\epsilon_{2}} \lambda \lambda^{-3 / 4+\epsilon_{2}} \lambda^{1 / 2}(\log \lambda) \lambda^{-\epsilon}(s-\lambda)^{-3 / 2} \lambda^{\prime-1} \lambda^{\prime-3 / 4} \lambda^{\epsilon_{2}} \lambda^{\prime} \lambda^{\prime-3 / 4} \lambda^{\epsilon_{2}} \lambda^{\prime 1 / 2} \\
& \cdot(\log \lambda) \lambda^{-\epsilon}\left(s-\lambda^{\prime}\right)^{-3 / 2} \\
& \lesssim(\log \lambda)^{2} \lambda^{-1+4 \epsilon_{2}-2 \epsilon} \lambda^{\prime-1}(s-\lambda)^{-3 / 2}\left(s-\lambda^{\prime}\right)^{-3 / 2} \text {. }
\end{aligned}
$$

Upon summing over $O\left(\lambda^{\epsilon+\epsilon_{3}}\right)$ indices $i=j+O(1)$, this leads to a small gain provided $\epsilon_{2}+\epsilon_{3} \ll \epsilon$, which we may arrange. This concludes the treatment of 5.53.

We next consider the contribution of the term

$$
\begin{aligned}
& \sum_{ \pm, \pm} \int_{T}^{\infty} t \int_{t}^{\infty}(v-1)(s) \\
& \cdot \int_{0}^{s / 2} \int_{-\infty}^{0}\left\langle\left(\begin{array}{c}
\chi>0\left|\tilde{U}^{(s)}\right|^{4}\left(\lambda^{\prime}, \cdot\right) \\
-\chi>0\left|\tilde{U}^{(s)}\right|^{4}\left(\lambda^{\prime}, \cdot\right)
\end{array}\right), \phi(x, \xi)\right\rangle \overline{\left\langle\beta_{1}\left(\lambda^{\prime}, \cdot\right),\left(e^{i x \xi}-e^{-i x \xi}\right) \underline{e}\right\rangle} d \xi d \lambda^{\prime} \\
& \cdot \int_{0}^{s / 2} \int_{-\infty}^{0}\left\langle\left(\begin{array}{c}
\chi>0\left|\tilde{U}^{(s)}\right|^{4}(\lambda, \cdot) \\
-\chi>0\left|\tilde{U}^{(s)}\right|^{4}(\lambda, \cdot)
\end{array}\right),\left(e^{i x \xi^{\prime}}-e^{-i x \xi^{\prime}}\right) \underline{e}\right\rangle \\
& \cdot \overline{\left\langle\beta_{1}(\lambda, \cdot),\left(e^{i x \xi^{\prime}}-e^{-i x \xi^{\prime}}\right) \underline{e}\right\rangle} d \xi^{\prime} d \lambda d s d t .
\end{aligned}
$$

We observe that we may innocuously include cutoffs $\phi_{<>\lambda^{\prime} \lambda^{\epsilon}}(|x|)$ simultaneously ${ }^{67}$ in front of

$$
\left(\begin{array}{c}
\chi_{>0}\left|\tilde{U}^{(s)}\right|^{4}\left(\lambda^{\prime}, \cdot\right) \\
-\chi_{>0}\left|\tilde{U}^{(s)}\right|^{4}\left(\lambda^{\prime}, \cdot\right)
\end{array}\right), \quad \beta_{1}\left(\lambda^{\prime}, \cdot\right) .
$$

Including the cutoff $\phi_{>\lambda^{\prime} \lambda^{\epsilon}}(|x|)$ clearly leads to the desired extra gain, while including the cutoff $\phi_{<\lambda^{\prime} \lambda^{\epsilon}}(|x|)$ allows us to restrict $e^{-i\left(s-\lambda^{\prime}\right) \mathcal{H}^{*}}\left(\mathcal{H}^{*}\right)^{-k} \psi_{\text {dis }}$ in $\beta_{1, \lambda^{\prime}}\left(\lambda^{\prime}, \cdot\right)$ to small

67 I.e., either both have the $<$ or the $>$ subscript. 
frequency. As we can always restrict the frequency of $e^{-i(s-\lambda) \mathcal{H}} \phi_{\text {dis }}$ in $\beta_{1}(\lambda, \cdot)$ away from zero, we have then achieved frequency separation and can argue as before in case (BC). This concludes case (CC) provided we have $\max \left\{\lambda, \lambda^{\prime}\right\}<s / 2$. The case $\max \left\{\lambda, \lambda^{\prime}\right\} \geq$ $s / 2$ is more elementary and omitted. We are now done with the proof of Lemma 5.11 . whence also the lemma before it.

We now continue with the proof of Proposition 5.10, note that the expressions

$$
\begin{aligned}
& \int_{T}^{\infty} t \int_{t}^{\infty}(v-1)^{a}(s)\left\langle\left(\begin{array}{c}
\tilde{U} \\
\tilde{\tilde{U}}
\end{array}\right)_{\mathrm{dis}}(s, \cdot), \phi\right\rangle d s d t, \quad a \geq 1, \\
& \int_{T}^{\infty} t \int_{t}^{\infty} \dot{v}(s)\left\langle\left(\begin{array}{c}
\tilde{U} \\
\overline{\tilde{U}}
\end{array}\right)_{\mathrm{dis}}(s, \cdot), \phi\right\rangle d s d t,
\end{aligned}
$$

can be treated exactly as above, using Lemma 5.11 and the relation 3.7. Thus up to terms which can either be estimated using Lemma 5.11 or else can even be absolutely integrated, there is only one potentially troublesome expression, namely

$$
\int_{T}^{\infty} t \int_{t}^{\infty}\left\langle\tilde{U}^{2}-\overline{\tilde{U}}^{2}, \phi\right\rangle d s d t
$$

where $\phi$ is an even time-independent Schwartz function:

(B) The contribution of the term $\int_{T}^{\infty} t \int_{t}^{\infty}\left\langle\tilde{U}^{2}-\overline{\tilde{U}}^{2}, \phi\right\rangle d s d t$. We handle this by the following lemma, which hinges on a symplectic structure:

Lemma 5.12. Let $\Gamma \in A_{[0, T)}^{(10)}, \Gamma=\left\{\left(\frac{\tilde{U}}{\tilde{U}}\right)_{\text {dis }}, \ldots\right\}$. Then, for $\tilde{T} \leq T$ and $\phi$ an even Schwartz function,

$$
\int_{\tilde{T}}^{T} t \int_{t}^{T}\left\langle\tilde{U}_{\mathrm{dis}}^{2}(s, \cdot)-\overline{\tilde{U}}_{\mathrm{dis}}^{2}(s, \cdot), \phi\right\rangle d s d t \lesssim \tilde{T}^{-1 / 2+\delta_{1}},
$$

provided $\delta_{1}$ is large enough in relation to $\delta_{2}, \delta_{3}$. Similarly,

$$
\int_{\tilde{T}}^{T} t^{2}\left\langle\tilde{U}_{\mathrm{dis}}^{2}(t, \cdot)-\overline{\tilde{U}}_{\mathrm{dis}}^{2}(t, \cdot), \phi\right\rangle d t \lesssim \tilde{T}^{-1 / 2+\delta_{1}}
$$

Both bounds are uniform in $T$.

Proof. It relies on identifying a special cancellation in this expression. We treat the first expression in detail, the second following the same reasoning. Also, we may put $T=\infty$. To begin with, write

$$
\left(\frac{\tilde{U}^{(s)}}{\tilde{U}^{(s)}}\right)_{\mathrm{dis}}=\sum_{ \pm} \int_{-\infty}^{\infty} e_{ \pm}(x, \xi) \mathcal{F}_{ \pm}\left(\frac{\tilde{U}^{(s)}}{\tilde{U}^{(s)}}\right)(\xi) d \xi
$$

This gets substituted into

$$
\left\langle\left(\tilde{U}_{\mathrm{dis}}^{(s)}\right)^{2}(s, \cdot)-\left(\overline{\tilde{U}_{\mathrm{dis}}^{(s)}}\right)^{2}(s, \cdot), \phi\right\rangle=\left\langle\left(\frac{\tilde{U}^{(s)}}{\tilde{U}^{(s)}}\right)_{\mathrm{dis}}^{t}\left(\begin{array}{cc}
1 & 0 \\
0 & -1
\end{array}\right)\left(\frac{\tilde{U}^{(s)}}{\tilde{U}^{(s)}}\right)_{\mathrm{dis}}, \phi\right\rangle .
$$


The key here is that

$$
\left\langle e_{+}^{t}(x, \xi)\left(\begin{array}{cc}
1 & 0 \\
0 & -1
\end{array}\right) e_{-}\left(x, \xi^{\prime}\right), \phi(x)\right\rangle=\left\langle e_{+}^{t}(x, \xi)\left(\begin{array}{cc}
0 & 1 \\
-1 & 0
\end{array}\right) e_{+}\left(x, \xi^{\prime}\right), \phi(x)\right\rangle,
$$

which is easily seen to vanish for $\xi=\xi^{\prime}$. Moreover, this also vanishes for $\xi=-\xi^{\prime}$, since then it is the inner product of an odd and an even function (use the fact that $e_{ \pm}(x,-\xi)=$ $\left.e_{ \pm}(-x, \xi)\right)$. To proceed, we now think of $\left(\frac{\tilde{U}^{(s)}}{\tilde{U}^{(s)}}\right)_{\mathrm{dis}}$ as a convex linear combination of functions $\left(\frac{\tilde{U}^{(s)}}{\tilde{U}^{(s)}}\right)_{i \text {, dis }}$, each of which satisfies a relation of type 3.27). Clearly, it suffices to substitute just one such function for each factor $\left(\frac{\tilde{U}^{(s)}}{\tilde{U}^{(s)}}\right)_{\text {dis }}$, and by abuse of notation, we shall denote these again as $\left(\frac{\tilde{U}^{(s)}}{\tilde{U}^{(s)}}\right)_{\text {dis }}$, as well as for the corresponding inputs of the Duhamel expression. Then substitute the latter into (5.58). We shall treat the most difficult term which results when we substitute the non-local source term for both factors, i.e., the expression

$$
\begin{aligned}
\sum_{ \pm, \pm} \int_{-\infty}^{\infty} \int_{-\infty}^{\infty} & \int_{T}^{\infty} t \int_{t}^{\infty}\left\langle e_{ \pm}(x, \xi)\left(\begin{array}{cc}
1 & 0 \\
0 & -1
\end{array}\right) e_{ \pm}\left(x, \xi^{\prime}\right), \phi(x)\right\rangle \\
& \cdot \int_{0}^{s} e^{\mp i(s-\lambda)\left(\xi^{2}+1\right)} \mathcal{F}_{ \pm}\left(\begin{array}{c}
\left|\tilde{U}^{(s)}\right|^{4} \tilde{U}^{(s)}(\lambda, \cdot) \\
-\left|\tilde{U}^{(s)}\right|^{4} \tilde{U}^{(s)}(\lambda, \cdot)
\end{array}\right)(\xi) d \lambda \\
& \cdot \int_{0}^{s} e^{\mp i\left(s-\lambda^{\prime}\right)\left(\xi^{2}+1\right)} \mathcal{F}_{ \pm}\left(\begin{array}{c}
\left|\tilde{U}^{(s)}\right|^{4} \tilde{U}^{(s)}\left(\lambda^{\prime}, \cdot\right) \\
-\left|\tilde{U}^{(s)}\right|^{4} \tilde{U}^{(s)}\left(\lambda^{\prime}, \cdot\right)
\end{array}\right)\left(\xi^{\prime}\right) d \lambda^{\prime} d \xi d \xi^{\prime} d s d t
\end{aligned}
$$

The remaining (local) source terms are handled by the exact same method but much easier, hence omitted. Then we focus on the most difficult case when the $s$-phases cancel each other, i.e., when there is a + and $a-$ sign. In order to render the Fourier transforms explicit, we write as usual

$$
\left(\begin{array}{c}
\left|\tilde{U}^{(s)}\right|^{4} \tilde{U}^{(s)}(\lambda, \cdot) \\
-\left|\tilde{U}^{(s)}\right|^{4} \tilde{U}^{(s)}(\lambda, \cdot)
\end{array}\right)=\chi_{>0}(x)\left(\begin{array}{c}
\left|\tilde{U}^{(s)}\right|^{4} \tilde{U}^{(s)}(\lambda, \cdot) \\
-\left|\tilde{U}^{(s)}\right|^{4} \tilde{U}^{(s)}(\lambda, \cdot)
\end{array}\right)+\chi_{\leq 0}(x)\left(\begin{array}{c}
\left|\tilde{U}^{(s)}\right|^{4} \tilde{U}^{(s)}(\lambda, \cdot) \\
-\left|\tilde{U}^{(s)}\right|^{4} \tilde{U}^{(s)}(\lambda, \cdot)
\end{array}\right),
$$

with a similar expression for the second factor in $\lambda^{\prime}$. Without loss of generality we shall then include the $\chi_{>0}(x)$-cutoff in both cases. Then we subdivide the $\left(\xi, \xi^{\prime}\right)$-plane into the four standard quadrants. If $\left(\xi, \xi^{\prime}\right)$ is in the first or third quadrant, observe that

$$
\frac{\left\langle\frac{e_{+}(x, \xi)}{\xi}\left(\begin{array}{cc}
0 & 1 \\
-1 & 0
\end{array}\right) \frac{e_{+}\left(x, \xi^{\prime}\right)}{\xi^{\prime}}, \phi(x)\right\rangle}{\xi-\xi^{\prime}}=\frac{\left\langle\left[\frac{e_{+}(x, \xi)}{\xi}-\frac{e_{+}\left(x, \xi^{\prime}\right)}{\xi^{\prime}}\right]\left(\begin{array}{cc}
0 & 1 \\
-1 & 0
\end{array}\right) \frac{e_{+}\left(x, \xi^{\prime}\right)}{\xi^{\prime}}, \phi(x)\right\rangle}{\xi-\xi^{\prime}},
$$

whence this is smooth and bounded with bounded derivatives in the interior of these quadrants, and continuous up to the boundary. If $\left(\xi, \xi^{\prime}\right)$ is in one of the other quadrants, we have

$$
\frac{\left\langle\frac{e_{+}(x, \xi)}{\xi}\left(\begin{array}{cc}
0 & 1 \\
-1 & 0
\end{array}\right) \frac{e_{+}\left(x, \xi^{\prime}\right)}{\xi^{\prime}}, \phi(x)\right\rangle}{\xi+\xi^{\prime}}=\frac{\left\langle\left[\frac{e_{+}(x, \xi)}{\xi}-\frac{e_{+}\left(x,-\xi^{\prime}\right)}{-\xi^{\prime}}\right]\left(\begin{array}{cc}
0 & 1 \\
-1 & 0
\end{array}\right) \frac{e_{+}\left(x, \xi^{\prime}\right)}{\xi^{\prime}}, \phi(x)\right\rangle}{\xi+\xi^{\prime}},
$$


whence the same comment applies. Now let $\left(\xi, \xi^{\prime}\right)$ be in the third quadrant. Then we can write

$$
\begin{aligned}
& \mathcal{F}_{+}\left(\begin{array}{c}
\chi>0(x)\left|\tilde{U}^{(s)}\right|^{4} \tilde{U}^{(s)}(\lambda, \cdot) \\
-\chi>0(x)\left|\tilde{U}^{(s)}\right|^{4} \tilde{U}^{(s)}(\lambda, \cdot)
\end{array}\right)(\xi) \\
& =\left\langle\left(\begin{array}{c}
\chi>0(x)\left|\tilde{U}^{(s)}\right|^{4} \tilde{U}^{(s)}(\lambda, \cdot) \\
-\chi>0(x)\left|\tilde{U}^{(s)}\right|^{4} \tilde{U}^{(s)}(\lambda, \cdot)
\end{array}\right),\left(e^{i x \xi}-e^{-i x \xi}\right) \underline{e}+(1+r(-\xi)) e^{-i x \xi} \underline{e}+\phi(x, \xi)\right\rangle,
\end{aligned}
$$

with a similar expression for $\mathcal{F}_{-}\left(\begin{array}{c}\chi>0(x)\left|\tilde{U}^{(s)}\right|^{4} \tilde{U}^{(s)}\left(\lambda^{\prime}, \cdot\right) \\ -\chi>0(x)\left|\tilde{U}^{(s)}\right|^{4} \tilde{U}^{(s)}\left(\lambda^{\prime}, \cdot\right)\end{array}\right)\left(\xi^{\prime}\right)$, where $\underline{\underline{e}}$ gets replaced by $\sigma_{1} \underline{\underline{e}}$. We shall treat in detail the contribution of $\left(e^{i x \xi}-e^{-i x \xi}\right) \underline{e}$, the other contributions being treated similarly. As usual, we need to distinguish between different frequency ranges: first assume $\max \left\{|\xi|,\left|\xi^{\prime}\right|\right\}>s^{\epsilon\left(\delta_{2}\right)}$, for suitable $\epsilon\left(\delta_{2}\right)$. For example assume $|\xi|>s^{\epsilon\left(\delta_{2}\right)}$, effected by means of a smooth cutoff $\phi_{>s^{\epsilon}}(\xi)$. Observe that then

$$
\begin{aligned}
& \left\langle\chi>0(x)\left(\begin{array}{c}
\left|\tilde{U}^{(s)}\right|^{4} \tilde{U}^{(s)}(\lambda, \cdot) \\
-\left|\tilde{U}^{(s)}\right|^{4} \tilde{U}^{(s)}(\lambda, \cdot)
\end{array}\right),\left(e^{i x \xi}-e^{-i x \xi}\right) \underline{e}\right\rangle \\
& =O\left(\frac{1}{s^{\epsilon}}\right)\left[\left\langle\delta_{0}(x)\left(\begin{array}{c}
\left|\tilde{U}^{(s)}\right|^{4} \tilde{U}^{(s)}(\lambda, \cdot) \\
-\left|\tilde{U}^{(s)}\right|^{4} \tilde{U}^{(s)}(\lambda, \cdot)
\end{array}\right),\left(e^{i x \xi}+e^{-i x \xi}\right) \underline{e}\right\rangle\right. \\
& \left.+\left\langle\chi>0(x) \partial_{x}\left(\begin{array}{c}
\left|\tilde{U}^{(s)}\right|^{4} \tilde{U}^{(s)}(\lambda, \cdot) \\
-\left|\tilde{U}^{(s)}\right|^{4} \tilde{U}^{(s)}(\lambda, \cdot)
\end{array}\right),\left(e^{i x \xi}+e^{-i x \xi}\right) \underline{e}\right\rangle\right] .
\end{aligned}
$$

For the boundary term, integrate by parts in $\xi$ in order to score arbitrary gains in $s$. For the second term, keep integrating by parts in $x$ until a boundary term results or else enough powers of $s$ are gained.

Thus we may now include smooth cutoffs $\phi_{<s^{\epsilon}}(\xi), \phi_{<s^{\epsilon}}\left(\xi^{\prime}\right)$. Commence with the case $\max \left\{\lambda, \lambda^{\prime}\right\}<s / 2$. We perform integrations by parts in $\xi, \xi^{\prime}$, and obtain the following list of integrals:

$$
\begin{aligned}
& A=\int_{-\infty}^{0} \int_{-\infty}^{0} \int_{T}^{\infty} t \int_{t}^{\infty} \phi_{<s^{\epsilon}}(\xi) \phi_{<s^{\epsilon}}\left(\xi^{\prime}\right)\left\langle\frac{e(x, \xi)}{\xi}\left(\begin{array}{cc}
0 & 1 \\
-1 & 0
\end{array}\right) \frac{e\left(x, \xi^{\prime}\right)}{\xi^{\prime}}, \phi(x)\right\rangle \\
& \cdot \int_{0}^{s / 2} \frac{1}{s-\lambda} e^{-i(s-\lambda)\left(\xi^{2}+1\right)} \partial_{\xi} \mathcal{F}_{+}\left(\begin{array}{c}
\chi>0(x)\left|\tilde{U}^{(s)}\right|^{4} \tilde{U}^{(s)}(\lambda, \cdot) \\
-\chi>0(x)\left|\tilde{U}^{(s)}\right|^{4} \tilde{U}^{(s)}(\lambda, \cdot)
\end{array}\right)(\xi) d \lambda \\
& \cdot \int_{0}^{s / 2} \frac{1}{s-\lambda^{\prime}} e^{+i(s-\lambda)\left(\xi^{\prime 2}+1\right)} \partial_{\xi^{\prime}} \mathcal{F}_{-}\left(\begin{array}{c}
\chi_{>0}(x)\left|\tilde{U}^{(s)}\right|^{4} \tilde{U}^{(s)}\left(\lambda^{\prime}, \cdot\right) \\
-\chi>0(x)\left|\tilde{U}^{(s)}\right|^{4} \tilde{U}^{(s)}\left(\lambda^{\prime}, \cdot\right)
\end{array}\right)\left(\xi^{\prime}\right) d \lambda^{\prime} d s d t d \xi d \xi^{\prime}, \\
& B=\int_{-\infty}^{0} \int_{-\infty}^{0} \int_{T}^{\infty} t \int_{t}^{\infty} \phi_{<s^{\epsilon}}(\xi) \phi_{<s^{\epsilon}}\left(\xi^{\prime}\right)\left\langle\partial_{\xi}\left[\frac{e(x, \xi)}{\xi}\right]\left(\begin{array}{cc}
0 & 1 \\
-1 & 0
\end{array}\right) \frac{e\left(x, \xi^{\prime}\right)}{\xi^{\prime}}, \phi(x)\right\rangle \\
& \cdot \int_{0}^{s / 2} \frac{1}{s-\lambda} e^{-i(s-\lambda)\left(\xi^{2}+1\right)} \mathcal{F}_{+}\left(\begin{array}{c}
\chi_{>0}(x)\left|\tilde{U}^{(s)}\right|^{4} \tilde{U}^{(s)}(\lambda, \cdot) \\
-\chi_{>0}(x)\left|\tilde{U}^{(s)}\right|^{4} \tilde{U}^{(s)}(\lambda, \cdot)
\end{array}\right)(\xi) d \lambda \\
& \cdot \int_{0}^{s / 2} \frac{1}{s-\lambda^{\prime}} e^{+i\left(s-\lambda^{\prime}\right)\left(\xi^{\prime 2}+1\right)} \partial_{\xi^{\prime}} \mathcal{F}_{-}\left(\begin{array}{c}
\chi_{>0}(x)\left|\tilde{U}^{(s)}\right|^{4} \tilde{U}^{(s)}\left(\lambda^{\prime}, \cdot\right) \\
-\chi_{>0}(x)\left|\tilde{U}^{(s)}\right|^{4} \tilde{U}^{(s)}\left(\lambda^{\prime}, \cdot\right)
\end{array}\right)\left(\xi^{\prime}\right) d \lambda^{\prime} d s d t d \xi d \xi^{\prime},
\end{aligned}
$$




$$
\begin{aligned}
& C=\int_{-\infty}^{0} \int_{-\infty}^{0} \int_{T}^{\infty} t \int_{t}^{\infty} \phi_{<s^{\epsilon}}(\xi) \phi_{<s^{\epsilon}}\left(\xi^{\prime}\right)\left\langle\frac{e(x, \xi)}{\xi}\left(\begin{array}{cc}
0 & 1 \\
-1 & 0
\end{array}\right) \partial_{\xi^{\prime}}\left[\frac{e\left(x, \xi^{\prime}\right)}{\xi^{\prime}}\right], \phi(x)\right\rangle \\
& \cdot \int_{0}^{s / 2} \frac{1}{s-\lambda} e^{-i(s-\lambda)\left(\xi^{2}+1\right)} \partial_{\xi} \mathcal{F}_{+}\left(\begin{array}{c}
\chi>0(x)\left|\tilde{U}^{(s)}\right|^{4} \tilde{U}^{(s)}(\lambda, \cdot) \\
-\chi>0(x)\left|\tilde{U}^{(s)}\right|^{4} \tilde{U}^{(s)}(\lambda, \cdot)
\end{array}\right)(\xi) d \lambda \\
& \cdot \int_{0}^{s / 2} \frac{1}{s-\lambda^{\prime}} e^{+i\left(s-\lambda^{\prime}\right)\left(\xi^{\prime 2}+1\right)} \mathcal{F}_{-}\left(\begin{array}{c}
\chi>0(x)\left|\tilde{U}^{(s)}\right|^{4} \tilde{U}^{(s)}\left(\lambda^{\prime}, \cdot\right) \\
-\chi>0(x)\left|\tilde{U}^{(s)}\right|^{4} \tilde{U}^{(s)}\left(\lambda^{\prime}, \cdot\right)
\end{array}\right)\left(\xi^{\prime}\right) d \lambda^{\prime} d s d t d \xi d \xi^{\prime}, \\
& D=\int_{-\infty}^{0} \int_{-\infty}^{0} \int_{T}^{\infty} t \int_{t}^{\infty} \phi_{<s^{\epsilon}}(\xi) \phi_{<s^{\epsilon}}\left(\xi^{\prime}\right)\left\langle\partial_{\xi}\left[\frac{e(x, \xi)}{\xi}\right]\left(\begin{array}{cc}
0 & 1 \\
-1 & 0
\end{array}\right) \partial_{\xi^{\prime}}\left[\frac{e\left(x, \xi^{\prime}\right)}{\xi^{\prime}}\right], \phi(x)\right\rangle \\
& \cdot \int_{0}^{s / 2} \frac{1}{s-\lambda} e^{-i(s-\lambda)\left(\xi^{2}+1\right)} \mathcal{F}_{+}\left(\begin{array}{c}
\chi>0(x)\left|\tilde{U}^{(s)}\right|^{4} \tilde{U}^{(s)}(\lambda, \cdot) \\
-\chi_{>0}(x)\left|\tilde{U}^{(s)}\right|^{4} \tilde{U}^{(s)}(\lambda, \cdot)
\end{array}\right)(\xi) d \lambda \\
& \cdot \int_{0}^{s / 2} \frac{1}{s-\lambda^{\prime}} e^{+i\left(s-\lambda^{\prime}\right)\left(\xi^{\prime 2}+1\right)} \mathcal{F}_{-}\left(\begin{array}{c}
\chi>0(x)\left|\tilde{U}^{(s)}\right|^{4} \tilde{U}^{(s)}\left(\lambda^{\prime}, \cdot\right) \\
-\chi>0(x)\left|\tilde{U}^{(s)}\right|^{4} \tilde{U}^{(s)}\left(\lambda^{\prime}, \cdot\right)
\end{array}\right)\left(\xi^{\prime}\right) d \lambda^{\prime} d s d t d \xi d \xi^{\prime} .
\end{aligned}
$$

We do not worry about the case when $\partial_{\xi}$ or $\partial_{\xi^{\prime}}$ falls on one of the cutoffs since then we have either $|\xi| \sim s^{\epsilon}$ or $\left|\xi^{\prime}\right| \sim s^{\epsilon}$, which case is treated as before. Observe that we can write

$$
\mathcal{F}_{+}\left(\begin{array}{c}
\left|\tilde{U}^{(s)}\right|^{4} \tilde{U}^{(s)}(\lambda, \cdot) \\
-\left|\tilde{U}^{(s)}\right|^{4} \tilde{U}^{(s)}(\lambda, \cdot)
\end{array}\right)(\xi)=\xi \int_{0}^{1} \partial_{\xi} \mathcal{F}_{+}\left(\begin{array}{c}
\left|\tilde{U}^{(s)}\right|^{4} \tilde{U}^{(s)}(\lambda, \cdot) \\
-\left|\tilde{U}^{(s)}\right|^{4} \tilde{U}^{(s)}(\lambda, \cdot)
\end{array}\right)(\alpha \xi) d \alpha
$$

Of course a similar identity applies to $\mathcal{F}_{-}(\ldots)$, whence we reduce to estimating expressions of the form

$$
\begin{aligned}
& \int_{-\infty}^{0} \int_{-\infty}^{0} \int_{T}^{\infty} t \int_{t}^{\infty} \phi_{<s^{\epsilon}}(\xi) \phi_{<s^{\epsilon}}\left(\xi^{\prime}\right) \xi g\left(\xi, \xi^{\prime}\right) \\
& \cdot \int_{0}^{s / 2} \frac{1}{s-\lambda} e^{-i(s-\lambda)\left(\xi^{2}+1\right)} \partial_{\xi} \mathcal{F}_{+}\left(\begin{array}{c}
\left|\tilde{U}^{(s)}\right|^{4} \tilde{U}^{(s)}(\lambda, \cdot) \\
-\left|\tilde{U}^{(s)}\right|^{4} \tilde{U}^{(s)}(\lambda, \cdot)
\end{array}\right)(\xi) d \lambda \\
& \cdot \int_{0}^{s / 2} \frac{1}{s-\lambda^{\prime}} e^{+i\left(s-\lambda^{\prime}\right)\left(\xi^{\prime 2}+1\right)} \partial_{\xi^{\prime}} \mathcal{F}_{-}\left(\begin{array}{c}
\left|\tilde{U}^{(s)}\right|^{4} \tilde{U}^{(s)}(\lambda, \cdot) \\
-\left|\tilde{U}^{(s)}\right|^{4} \tilde{U}^{(s)}\left(\lambda^{\prime}, \cdot\right)
\end{array}\right)\left(\xi^{\prime}\right) d \lambda^{\prime} d s d t d \xi d \xi^{\prime},
\end{aligned}
$$

where the function $g\left(\xi, \xi^{\prime}\right)$ is smooth and bounded in the interior of the third quadrant as well as continuous up to the boundary. Expand as usual

$$
\begin{aligned}
& \partial_{\xi} \mathcal{F}_{+}\left(\begin{array}{c}
\chi>0(x)\left|\tilde{U}^{(s)}\right|{ }^{4} \tilde{U}^{(s)}(\lambda, \cdot) \\
-\chi>0(x)\left|\tilde{U}^{(s)}\right|^{4} \tilde{U}^{(s)}(\lambda, \cdot)
\end{array}\right)(\xi) \\
& =\left\langle\left(\begin{array}{c}
x \chi>0(x)\left|\tilde{U}^{(s)}\right|^{4} \tilde{U}^{(s)}(\lambda, \cdot) \\
-x \chi_{>0}(x)\left|\tilde{U}^{(s)}\right|^{4} \tilde{U}^{(s)}(\lambda, \cdot)
\end{array}\right),\left(e^{i x \xi}+e^{-i x \xi}\right) \underline{e}\right\rangle+\cdots
\end{aligned}
$$

where ... represent terms that can be treated similarly. Now assume that we localize $x$ to dyadic range $|x| \sim 2^{k}, k \geq 0$. If we then have $|\xi|>\max \left\{s^{-1 / 2+}, s^{-1-} 2^{k}\right\}$, effected by means of a smooth cutoff, we obtain arbitrary gains in $s$ by integration by parts in $\xi$. 
Thus we shall now include a localizer $\phi_{<\max \left\{s^{-1 / 2+}, s^{-1-} 2^{k}\right\}}(\xi)$ upon localizing $x$ to dyadic range $|x| \sim 2^{k}$, i.e., we reduce to considering

$$
\begin{aligned}
& \sum_{k} \int_{-\infty}^{0} \int_{-\infty}^{0} \int_{T}^{\infty} t \int_{t}^{\infty} \phi_{<\max \left\{s^{-1 / 2+}, s^{\left.-1-22^{k}\right\}}\right.}(\xi) \phi_{<s^{\epsilon}}(\xi) \phi_{<s^{\epsilon}}\left(\xi^{\prime}\right) \xi g\left(\xi, \xi^{\prime}\right) \\
& \cdot \int_{0}^{s / 2} \frac{1}{s-\lambda} e^{-i(s-\lambda)\left(\xi^{2}+1\right)}\left\langle\left(\begin{array}{c}
\phi_{\sim 2^{k}}(x) x\left|\tilde{U}^{(s)}\right|^{4} \tilde{U}^{(s)}(\lambda, \cdot) \\
-\phi_{\sim 2^{k}}(x) x\left|\tilde{U}^{(s)}\right|^{4} \tilde{U}^{(s)}(\lambda, \cdot)
\end{array}\right),\left(e^{i x \xi}+e^{-i x \xi}\right) \underline{e}\right\rangle d \lambda \\
& \left.\cdot \int_{0}^{s / 2} \frac{1}{s-\lambda^{\prime}} e^{+i\left(s-\lambda^{\prime}\right)\left(\xi^{\prime 2}+1\right)}\left(\begin{array}{c}
x\left|\tilde{U}^{(s)}\right|^{4} \tilde{U}^{(s)}(\lambda, \cdot) \\
-x\left|\tilde{U}^{(s)}\right|^{4} \tilde{U}^{(s)}(\lambda, \cdot)
\end{array}\right),\left(e^{i x \xi^{\prime}}+e^{-i x \xi^{\prime}}\right) \sigma_{1} \underline{e}\right) d \lambda^{\prime} d s d t d \xi d \xi^{\prime} .
\end{aligned}
$$

Note that summing over $k$ will amount to an extra $\log s$ at most, whence we shall safely discard this summation. Our strategy will be to perform an integration by parts in $\left\langle\left(\begin{array}{c}\phi_{\sim 2^{k}}(x)\left|\tilde{U}^{(s)}\right|^{4} \tilde{U}^{(s)}(\lambda, \cdot) \\ -\phi_{\sim 2^{k}}(x)\left|\tilde{U}^{(s)}\right|^{4} \tilde{U}^{(s)}(\lambda, \cdot)\end{array}\right),\left(e^{i x \xi}+e^{-i x \xi}\right) \underline{e}\right\rangle$. For this to be useful, though, we need to achieve some preliminary reductions in the last factor $\tilde{U}^{(s)}$, just as in the proof of SLDE. Recall that we can write

$$
\tilde{U}^{(s)}(\lambda, \cdot)=\int_{0}^{\lambda} \sqrt{\lambda-\mu}^{-1} \int_{-\infty}^{\infty} e^{\frac{(y-z)^{2}}{i(\lambda-\mu)}} g(\mu, z) d z d \mu
$$

where $g(\mu, z)=\left|\tilde{U}^{(s)}\right|^{4} \tilde{U}^{(s)}(\mu, z)+\cdots$. Decompose

$$
\left|\tilde{U}^{(s)}\right|^{4} \tilde{U}^{(s)}(\mu, z)=\chi_{<2^{k}}(\mu)\left|\tilde{U}^{(s)}\right|^{4} \tilde{U}^{(s)}(\mu, z)+\chi_{\geq 2^{k}}(\mu)\left|\tilde{U}^{(s)}\right|^{4} \tilde{U}^{(s)}(\mu, z) .
$$

Then observe that

$$
\left\|\int_{0}^{\lambda} \sqrt{\lambda-\mu}^{-1} \int_{-\infty}^{\infty} e^{\frac{(y-z)^{2}}{i(\lambda-\mu)}} \chi_{\geq 2^{k}}(\mu)\left|\tilde{U}^{(s)}\right|^{4} \tilde{U}^{(s)}(\mu, z) d z d \mu\right\|_{L_{y}^{2}} \lesssim \int_{2^{k}}^{\lambda} \mu^{-2} d \mu \lesssim 2^{-k} .
$$

Similarly, we have

$$
\begin{aligned}
\| \int_{0}^{\lambda} \sqrt{\lambda-\mu}^{-1} & \int_{-\infty}^{\infty} e^{\frac{(y-z)^{2}}{i(\lambda-\mu)}} \chi_{<2^{k}}(\mu) \chi_{\geq 2} 2^{k-10}(z)\left|\tilde{U}^{(s)}\right|^{4} \tilde{U}^{(s)}(\mu, z) d z d \mu \|_{L_{y}^{2}} \\
& \lesssim \int_{0}^{\lambda}\left\|\chi_{\geq s^{k}}(z) \frac{2 i \mu \partial_{z} \tilde{U}^{(s)}(\mu, z)-C \tilde{U}^{(s)}(\mu, z)}{z}\right\|_{L_{z}^{2}} \mu^{-2} d \mu \lesssim(\log \lambda) 2^{-k} .
\end{aligned}
$$

One obtains similar estimates if one substitutes the remaining local terms in $g(\mu, z)$, localized to $|z|>2^{k-10}$, in the preceding integral. Thus if we substitute

$$
\begin{aligned}
V^{(s)}(\lambda, y):= & \int_{0}^{\lambda} \sqrt{\lambda-\mu}^{-1} \int_{-\infty}^{\infty} e^{\frac{(y-z)^{2}}{i(\lambda-\mu)}}\left[\chi_{\geq 2^{k}}(\mu)\left|\tilde{U}^{(s)}\right|^{4} \tilde{U}^{(s)}(\mu, z)\right. \\
& \left.+\chi_{<2^{k}}(\mu) \chi_{\geq 2^{k-10}}(z)\left|\tilde{U}^{(s)}\right|^{4} \tilde{U}^{(s)}(\mu, z)+\chi_{\geq 2^{k-10}}(z) \ldots\right] d z d \mu
\end{aligned}
$$


instead of $\tilde{U}^{(s)}$ for the last factor in $\left|\tilde{U}^{(s)}\right|^{4} \tilde{U}^{(s)}(\lambda, y)$, we get

$$
\left\|\phi_{\sim 2^{k}}(x) x\left|\tilde{U}^{(s)}\right|^{4} V^{(s)}(\lambda, x)\right\|_{L_{x}^{1}} \lesssim \lambda^{-3 / 2} .
$$

We now show how this suffices to control

$$
\begin{aligned}
& \int_{-\infty}^{0} \int_{-\infty}^{0} \int_{T}^{\infty} t \int_{t}^{\infty} \phi_{<s^{\epsilon}}(\xi) \phi_{<s^{\epsilon}}\left(\xi^{\prime}\right) \phi_{<\max \left\{s^{-1 / 2+}, s^{-1-} 2^{k}\right\}}(\xi) \xi g\left(\xi, \xi^{\prime}\right) \\
& \cdot \int_{0}^{s / 2} \frac{1}{s-\lambda} e^{-i(s-\lambda)\left(\xi^{2}+1\right)}\left\langle\left(\begin{array}{c}
\left.x \phi_{\sim 2^{k}}(x)\left|\tilde{U}^{(s)}\right|^{4} V^{(s)} \lambda, x\right) \\
-x \phi \sim 2^{k}(x)\left|\tilde{U}^{(s)}\right|^{4} V^{(s)}(\lambda, \cdot)
\end{array}\right),\left(e^{i x \xi}+e^{-i x \xi}\right) \underline{e}\right\rangle d \lambda \\
& \cdot \int_{0}^{s / 2} \frac{1}{s-\lambda^{\prime}} e^{+i(s-\lambda)\left(\xi^{\prime 2}+1\right)}\left(\left(\begin{array}{c}
x\left|\tilde{U}^{(s)}\right|^{4} \tilde{V}^{(s)}\left(\lambda^{\prime}, \cdot\right) \\
-x\left|\tilde{U}^{(s)}\right|^{4} \tilde{V}^{(s)}\left(\lambda^{\prime}, \cdot\right)
\end{array}\right),\left(e^{i x \xi^{\prime}}+e^{-i x \xi^{\prime}}\right) \sigma_{1} \underline{e}\right) d \lambda^{\prime} d s d t d \xi d \xi^{\prime}
\end{aligned}
$$

where $\tilde{V}^{(s)}\left(\lambda^{\prime}, \cdot\right)$ is defined analogously. Thus we can estimate this by

$$
\begin{gathered}
\int_{T}^{\infty} t \int_{t}^{\infty} \max \left\{s^{-1 / 2+}, s^{-1-} 2^{k}\right\} \int_{0}^{s / 2} \frac{1}{(s-\lambda)^{3 / 2}}\left\|\left(\begin{array}{c}
\left.x \phi_{\sim 2^{k}}(x)\left|\tilde{U}^{(s)}\right|^{4} V^{(s)} \lambda, x\right) \\
-x \phi_{\sim 2^{k}}(x)\left|\tilde{U}^{(s)}\right|^{4} V^{(s)}(\lambda, \cdot)
\end{array}\right)\right\|_{L_{x}^{1}} d \lambda \\
\cdot \int_{0}^{s / 2} \frac{1}{\left(s-\lambda^{\prime}\right)^{3 / 2}}\left\|\left(\begin{array}{c}
x\left|\tilde{U}^{(s)}\right|^{4} \tilde{V}^{(s)}\left(\lambda^{\prime}, \cdot\right) \\
-x\left|\tilde{U}^{(s)}\right|^{4} \tilde{V}^{(s)}\left(\lambda^{\prime}, \cdot\right)
\end{array}\right)\right\|_{L_{x}^{1}} d \lambda^{\prime} d s d t \\
\qquad \int_{T}^{\infty} t \int_{t}^{\infty} s^{-1 /(2+)} s^{-3} d s d t \lesssim T^{-1 /(2+)} .
\end{gathered}
$$

We are exploiting here the pseudo-conformal almost conservation, which implies that

$$
\left.\| x \phi_{\sim 2^{k}}(x)\left|\tilde{U}^{(s)}\right|^{4} V^{(s)} \lambda, x\right) \|_{L_{x}^{1}} \lesssim \frac{\lambda}{2^{k}} \lambda^{-3 / 2} .
$$

Thus we now replace at least one of $\tilde{U}^{(s)}(\lambda, \cdot), \tilde{U}^{(s)}\left(\lambda^{\prime}, \cdot\right)$ by

$$
\begin{aligned}
& \tilde{W}^{(s)}(\lambda, \cdot) \\
& \quad:=\int_{-\infty}^{\infty} \int_{0}^{\lambda} \frac{1}{\sqrt{\lambda-\mu}} e^{\frac{(y-z)^{2}}{i(\lambda-\mu)}}\left[\chi_{<2^{k}}(\mu) \chi_{<2^{k-10}}(z)\left|\tilde{U}^{(s)}\right|^{4} \tilde{U}^{(s)}(\mu, z)+\cdots\right] d \mu d z \quad \text { etc. }
\end{aligned}
$$

Then we decouple the $\xi, \xi^{\prime}$ variables in 5.60 , which can be achieved by means of discrete Fourier expansion:

$$
\phi_{<s^{\epsilon}}(|\xi|) \phi_{<s^{\epsilon}}\left(\left|\xi^{\prime}\right|\right) \xi g\left(\xi, \xi^{\prime}\right)=\xi \sum_{n, m \in s^{-\epsilon} \mathbb{Z}} a_{n m} e^{i n \xi+i m \xi^{\prime}}, \quad\left|a_{n m}\right| \lesssim\left[s^{\epsilon}|n|+s^{\epsilon}|m|\right]^{-N}
$$

Consider the case when we replace the fifth $\tilde{U}^{(s)}(\lambda, \cdot)$ by $\tilde{W}^{(s)}(\lambda, \cdot)$. We are thus led to estimating contributions of the form

$$
\int_{-\infty}^{0} \xi \phi_{<s^{\epsilon}}(\xi) e^{i(s-\lambda)\left(\xi^{2}+1\right)}\left\langle\chi_{>0}(x) \phi_{\sim 2^{k}}(x) x\left|\tilde{U}^{(s)}\right|{ }^{4} \tilde{W}^{(s)}(\lambda, \cdot),\left(e^{i(x+n) \xi}+e^{-i(x-n) \xi}\right) \underline{e}\right\rangle d \xi .
$$

We shall put $n=0$ since the other cases are dealt with similarly. We treat here the contribution of $e^{-i x \xi}$, the one of $e^{+i x \xi}$ being treated similarly. Switch the order of integration in this, and introduce the new variable $\tilde{\xi}:=\sqrt{s-\lambda} \xi+\frac{x}{2 \sqrt{s-\lambda}}$. Then we can rewrite the 
preceding expression as

$$
\begin{aligned}
\frac{1}{\sqrt{s-\lambda}} \int_{0}^{\infty} \int_{-\infty}^{\frac{x}{2 \sqrt{s-\lambda}}} \phi_{<s^{\epsilon}}\left(\frac{\tilde{\xi}-\frac{x}{2 \sqrt{s-\lambda}}}{\sqrt{s-\lambda}}\right) \frac{\tilde{\xi}-\frac{x}{2 \sqrt{s-\lambda}}}{\sqrt{s-\lambda}} \\
\cdot e^{i \tilde{\xi}^{2}} e^{\frac{x^{2}}{4 i(s-\lambda)} x \phi_{\sim 2^{k}}(x)\left|\tilde{U}^{(s)}\right|^{4} \tilde{W}^{(s)}(\lambda, \cdot) d \tilde{\xi} d x}
\end{aligned}
$$

Now perform an integration by parts in the $x$-variable, and replace the preceding by the sum of multiples of the following expressions (as well as equivalent terms):

$$
\begin{gathered}
\frac{1}{\sqrt{s-\lambda}} \int_{0}^{\infty} \int_{-\infty}^{\frac{x}{2 \sqrt{s-\lambda}}} \partial_{x}\left[\phi_{<s^{\epsilon}}\left(\frac{\tilde{\xi}-\frac{x}{2 \sqrt{s-\lambda}}}{\sqrt{s-\lambda}}\right) \frac{\tilde{\xi}-\frac{x}{2 \sqrt{s-\lambda}}}{\sqrt{s-\lambda}}\right] e^{i \tilde{\xi}^{2}} d \tilde{\xi} \\
\cdot x \phi_{\sim 2^{k}}(x)\left|\tilde{U}^{(s)}\right|^{4}\left[\int_{x}^{\infty} e^{\frac{y^{2}}{4 i(s-\lambda)}} \tilde{W}^{(s)}(\lambda, y) d y\right] d x, \\
\frac{1}{\sqrt{s-\lambda}} \int_{0}^{\infty} \int_{-\infty}^{\frac{x}{2 \sqrt{s-\lambda}}}\left[\phi_{<s^{\epsilon}}\left(\frac{\tilde{\xi}-\frac{x}{2 \sqrt{s-\lambda}}}{\sqrt{s-\lambda}}\right) \frac{\tilde{\xi}-\frac{x}{2 \sqrt{s-\lambda}}}{\sqrt{s-\lambda}}\right] e^{i \tilde{\xi}^{2}} d \tilde{\xi} \\
\frac{1}{\sqrt{s-\lambda}} \int_{0}^{\infty} \int_{-\infty}^{\frac{x}{2 \sqrt{s-\lambda}}}\left[\phi_{<2^{k}}\left(\frac{\tilde{\xi}) \mid \tilde{U}^{(s)}}{\left.\right|^{4}\left[\int_{x}^{\infty} e^{\frac{y^{2}}{4 i(s-\lambda)}} \tilde{W}^{(s)}(\lambda, y) d y\right] d x,} \frac{x}{\sqrt{s-\lambda}}\right) \frac{\tilde{\xi}-\frac{x}{2 \sqrt{s-\lambda}}}{\sqrt{s-\lambda}}\right] e^{i \tilde{\xi}^{2}} d \tilde{\xi} \\
\cdot x \phi_{\sim 2^{k}}(x) \times \partial_{x}\left[\left|\tilde{U}^{(s)}\right|^{4}(\lambda, x)\right]\left[\int_{x}^{\infty} e^{\frac{y^{2}}{4 i(s-\lambda)}} \tilde{W}^{(s)}(\lambda, y) d y\right] d x .
\end{gathered}
$$

Now write

$$
\tilde{W}^{(s)}(\lambda, y)=\int_{-\infty}^{\infty} \int_{0}^{\lambda} \frac{1}{\sqrt{\lambda-\mu}} e^{\frac{(y-z)^{2}}{i(\lambda-\mu)}} g(\mu, z) d \mu d z,
$$

where we have $|z|<2^{k-10}$ on the support of $g(\mu, z)$. Thus we obtain

$$
\begin{aligned}
& \int_{x}^{\infty} e^{\frac{y^{2}}{4 i(s-\lambda)}} \tilde{W}^{(s)}(\lambda, y) d y=\int_{-\infty}^{\infty} \int_{x}^{\infty} \int_{0}^{\lambda} \frac{1}{\sqrt{\lambda-\mu}} e^{\frac{y^{2}}{4 i(s-\lambda)}} e^{\frac{(y-z)^{2}}{i(\lambda-\mu)}} g(\mu, z) d \mu d y d z \\
& =\int_{-\infty}^{\infty} \int_{0}^{\lambda} O\left(\frac{1}{\sqrt{\lambda-\mu} \sqrt{\frac{1}{4(s-\lambda)}+\frac{1}{\lambda-\mu}}} \frac{1}{x \sqrt{\frac{1}{4(s-\lambda)}+\frac{1}{\lambda-\mu}}-\frac{z}{(\lambda-\mu) \sqrt{\frac{1}{4(s-\lambda)}+\frac{1}{\lambda-\mu}}}}\right) \\
& \text { - } g(\mu, z) d \mu \phi_{<2^{k-10}}(|z|) d z \text {, }
\end{aligned}
$$

which is seen for $x \sim 2^{k}$ to be of order $(x / \sqrt{\lambda})^{-1}$, upon using the definition of $g(\mu, z)$. Now plug this into 5.61. For example, we get

$$
\begin{aligned}
& \mid \frac{1}{\sqrt{s-\lambda}} \int_{0}^{\infty} \int_{-\infty}^{\frac{x}{2 \sqrt{s-\lambda}}} \frac{1}{s-\lambda}\left[\phi_{<s^{\prime}}^{\prime}\left(\frac{\tilde{\xi}-\frac{x}{2 \sqrt{s-\lambda}}}{\sqrt{s-\lambda}}\right) \frac{x}{2(s-\lambda)}\right] e^{i \tilde{\xi}^{2}} d \tilde{\xi} \\
& \cdot x \phi_{\sim 2^{k}}(x)\left|\tilde{U}^{(s)}\right|^{4}\left[\int_{x}^{\infty} e^{\frac{y^{2}}{4 i(s-\lambda)}} \tilde{W}^{(s)}(\lambda, y) d y\right] d x \mid \lesssim \frac{1}{\sqrt{s-\lambda}} \frac{1}{(s-\lambda)^{2}} \sqrt{\lambda} \lambda^{\epsilon\left(\delta_{2}\right)} .
\end{aligned}
$$


Observe that we are using pseudo-conformal almost conservation here. Integrating over $\lambda<s / 2$ results in the upper bound $\lesssim s^{-1}$. The remaining contributions to (5.61) (Leibniz rule) are treated similarly, as is the contribution of 5.62). Now consider 5.63. Here we invoke the same trick as in the proof of SLDE:

$$
\partial_{x}\left[\left|\tilde{U}^{(s)}\right|^{2}(\lambda, x)\right]=\frac{1}{i \lambda}\left[C \tilde{U}^{(s)} \overline{\tilde{U}^{(s)}}(\lambda, x)-\tilde{U}^{(s)} \overline{C \tilde{U}^{(s)}}(\lambda, x)\right] .
$$

For example, we can estimate

$$
\begin{aligned}
& \mid \frac{1}{\sqrt{s-\lambda}} \int_{0}^{\infty} \int_{-\infty}^{\frac{x}{2 \sqrt{s-\lambda}}} \phi_{<s^{\epsilon}}\left(\frac{\tilde{\xi}-\frac{x}{2 \sqrt{s-\lambda}}}{\sqrt{s-\lambda}}\right) \frac{x}{s-\lambda} e^{i \tilde{\xi}^{2}} d \tilde{\xi} \\
& \qquad x \phi_{\sim 2^{k}}(x) \partial_{x}\left[\left|\tilde{U}^{(s)}\right|^{4}(\lambda, x)\right]\left[\int_{x}^{\infty} e^{\left.\frac{y^{2}}{4 i(s-\lambda)} \tilde{W}^{(s)}(\lambda, y) d y\right] d x \mid}\right. \\
& \lesssim \mid \frac{1}{\sqrt{s-\lambda}} \int_{0}^{\infty} \int_{-\infty}^{\frac{x}{2 \sqrt{s-\lambda}}}\left[\phi_{<s^{\epsilon}}\left(\frac{\tilde{\xi}-\frac{x}{2 \sqrt{s-\lambda}}}{\sqrt{s-\lambda}}\right) \frac{1}{(s-\lambda)} e^{i \tilde{\xi}^{2}} d \tilde{\xi}\right. \\
& \left.\cdot \phi_{\sim 2^{k}}(x) \frac{1}{\lambda}\left|C \tilde{U}^{(s)}\left\|x \tilde{U}^{(s)}\right\| \tilde{U}^{(s)}\right|^{2}(\lambda, x)\right] \sqrt{\lambda} d \tilde{\xi} d x \mid \lesssim(s-\lambda)^{-3 / 2} \sqrt{\lambda}^{-1} \lambda^{\epsilon\left(\delta_{2}\right)},
\end{aligned}
$$

which upon integration over $\lambda<s / 2$ again results in the estimate $s^{-1+}$. The second contribution to 5.63 is treated similarly.

Keeping in mind that we need to eventually estimate (5.60), we next consider the expression

$$
\int_{-\infty}^{0} \phi_{<s^{\epsilon}}\left(\xi^{\prime}\right) e^{i(s-\lambda)\left(\xi^{\prime 2}+1\right)}\left\langle\chi_{>0}(x) \phi_{\sim 2^{k}}(x) x\left|\tilde{U}^{(s)}\right|^{4} \tilde{W}^{(s)}\left(\lambda^{\prime}, x\right),\left(e^{i x \xi^{\prime}}+e^{-i x \xi^{\prime}}\right) \underline{e}\right\rangle d \xi^{\prime} .
$$

One proceeds analogously and obtains expressions as in 5.61, 5.62, (5.63) but without the factor $\left(\tilde{\xi^{\prime}}-\frac{x}{2 \sqrt{s-\lambda^{\prime}}}\right) / \sqrt{s-\lambda^{\prime}}$. One then has to argue somewhat differently for the expression

$$
\begin{aligned}
\frac{1}{\sqrt{s-\lambda^{\prime}}} \int_{0}^{\infty} \int_{-\infty}^{\frac{x}{2 \sqrt{s-\lambda^{\prime}}}} \phi_{<s^{\epsilon}}\left(\frac{\tilde{\xi}-\frac{x}{2 \sqrt{s-\lambda^{\prime}}}}{\sqrt{s-\lambda^{\prime}}}\right) e^{i \tilde{\xi}^{2}} d \tilde{\xi} \\
\cdot \phi_{\sim 2^{k}}(x)\left|\tilde{U}^{(s)}\right|^{4}\left(\lambda^{\prime}, x\right)\left[\int_{x}^{\infty} e^{\frac{y^{2}}{4 i\left(s-\lambda^{\prime}\right)}} \tilde{W}^{(s)}\left(\lambda^{\prime}, y\right) d y\right] d x .
\end{aligned}
$$

Here we use the estimate

$$
\left|\tilde{U}^{(s)}\right|\left(\lambda^{\prime}, x\right)\left[\frac{\langle x\rangle^{1 / 2}}{\sqrt{\lambda^{\prime}}}\right]^{-1} \lesssim \sqrt{\lambda^{\prime}} \lambda^{\prime-1+\epsilon\left(\delta_{2}\right)},
$$

whence we can bound the above expression by

$$
\frac{1}{\sqrt{s-\lambda^{\prime}}} \lambda^{\prime-3 / 2+\epsilon\left(\delta_{2}\right)}
$$


Integrating over $\lambda^{\prime}<s / 2$ results in the upper bound $\sqrt{s}^{-1+\epsilon\left(\delta_{2}\right)}$. Combining all these estimates, we now obtain

$$
\begin{aligned}
& \mid \sum_{k} \int_{-\infty}^{0} \int_{-\infty}^{0} \int_{T}^{\infty} t \int_{t}^{\infty} \phi_{<s^{\epsilon}}(\xi) \phi_{<s^{\epsilon}}\left(\xi^{\prime}\right) \xi g\left(\xi, \xi^{\prime}\right) \\
& \cdot \int_{0}^{s / 2} \frac{1}{s-\lambda} e^{-i(s-\lambda)\left(\xi^{2}+1\right)}\left\langle\left(\begin{array}{c}
\phi_{\sim 2^{k}}(x)\left|\tilde{U}^{(s)}\right|^{4} \tilde{W}^{(s)}(\lambda, \cdot) \\
-\phi_{\sim 2^{k}}(x)\left|\tilde{U}^{(s)}\right|^{4} \tilde{W}^{(s)}(\lambda, \cdot)
\end{array}\right),\left(e^{i x \xi}+e^{-i x \xi}\right) \underline{e}\right\rangle d \lambda \\
& \cdot \int_{0}^{s / 2} \frac{1}{s-\lambda^{\prime}} e^{+i(s-\lambda)\left(\xi^{\prime 2}+1\right)}\left\langle\left(\begin{array}{c}
x\left|\tilde{U}^{(s)}\right|^{4} \tilde{W}^{(s)}(\lambda, \cdot) \\
-x\left|\tilde{U}^{(s)}\right|^{4} \tilde{W}^{(s)}(\lambda, \cdot)
\end{array}\right),\left(e^{i x \xi^{\prime}}+e^{-i x \xi^{\prime}}\right) \sigma_{1} e \underline{e}\right\rangle d \lambda^{\prime} d s d t d \xi d \xi^{\prime} \mid \\
& \lesssim T^{-1 /(2+)}, \\
& \mid \sum_{k} \int_{-\infty}^{0} \int_{-\infty}^{0} \int_{T}^{\infty} t \int_{t}^{\infty} \phi_{<s^{\epsilon}}(\xi) \phi_{<s^{\epsilon}}\left(\xi^{\prime}\right) \xi g\left(\xi, \xi^{\prime}\right) \\
& \cdot \int_{0}^{s / 2} \frac{1}{s-\lambda} e^{-i(s-\lambda)\left(\xi^{2}+1\right)}\left\langle\left(\begin{array}{c}
\phi_{\sim 2^{k}}(x)\left|\tilde{U}^{(s)}\right|^{4} \tilde{W}^{(s)}(\lambda, \cdot) \\
-\phi_{\sim 2^{k}}(x)\left|\tilde{U}^{(s)}\right|^{4} \tilde{W}^{(s)}(\lambda, \cdot)
\end{array}\right),\left(e^{i x \xi}+e^{-i x \xi}\right) \underline{e}\right\rangle d \lambda \\
& \cdot \int_{0}^{s / 2} \frac{1}{s-\lambda^{\prime}} e^{+i(s-\lambda)\left(\xi^{\prime 2}+1\right)}\left\langle\left(\begin{array}{c}
x\left|\tilde{U}^{(s)}\right|^{4} \tilde{V}^{(s)}(\lambda, \cdot) \\
-x\left|\tilde{U}^{(s)}\right|^{4} \tilde{V}^{(s)}(\lambda, \cdot)
\end{array}\right),\left(e^{i x \xi^{\prime}}+e^{-i x \xi^{\prime}}\right) \sigma_{1} \underline{e}\right\rangle d \lambda^{\prime} d s d t d \xi d \xi^{\prime} \mid \\
& \lesssim T^{-1 /(2+)}, \\
& \mid \sum_{k} \int_{-\infty}^{0} \int_{-\infty}^{0} \int_{T}^{\infty} t \int_{t}^{\infty} \phi_{<s^{\epsilon}}(\xi) \phi_{<s^{\epsilon}}\left(\xi^{\prime}\right) \xi g\left(\xi, \xi^{\prime}\right) \\
& \cdot \int_{0}^{s / 2} \frac{1}{s-\lambda} e^{-i(s-\lambda)\left(\xi^{2}+1\right)}\left(\left(\begin{array}{c}
\phi_{\sim 2^{k}}(x)\left|\tilde{U}^{(s)}\right|^{4} \tilde{V}^{(s)}(\lambda, \cdot) \\
-\phi_{\sim 2^{k}}(x)\left|\tilde{U}^{(s)}\right|^{4} \tilde{V}^{(s)}(\lambda, \cdot)
\end{array}\right),\left(e^{i x \xi}+e^{-i x \xi}\right) \underline{e}\right\rangle d \lambda \\
& \cdot \int_{0}^{s / 2} \frac{1}{s-\lambda^{\prime}} e^{+i(s-\lambda)\left(\xi^{\prime 2}+1\right)}\left\langle\left(\begin{array}{c}
x\left|\tilde{U}^{(s)}\right|^{4} \tilde{W}^{(s)}(\lambda, \cdot) \\
-x\left|\tilde{U}^{(s)}\right|^{4} \tilde{W}^{(s)}(\lambda, \cdot)
\end{array}\right),\left(e^{i x \xi^{\prime}}+e^{-i x \xi^{\prime}}\right) \sigma_{1} \underline{e}\right\rangle d \lambda^{\prime} d s d t d \xi d \xi^{\prime} \mid \\
& \lesssim T^{-1 /(2+)},
\end{aligned}
$$

which together with 5.59 implies

$$
\begin{aligned}
& \mid \sum_{k} \int_{-\infty}^{0} \int_{-\infty}^{0} \int_{T}^{\infty} t \int_{t}^{\infty} \phi_{<s^{\epsilon}}(\xi) \phi_{<s^{\epsilon}}\left(\xi^{\prime}\right) \xi g\left(\xi, \xi^{\prime}\right) \\
& \cdot \int_{0}^{s / 2} \frac{1}{s-\lambda} e^{-i(s-\lambda)\left(\xi^{2}+1\right)}\left\langle\left(\begin{array}{c}
\phi_{\sim 2^{k}}(x)\left|\tilde{U}^{(s)}\right|^{4} \tilde{U}^{(s)}(\lambda, \cdot) \\
-\phi_{\sim 2^{k}}(x)\left|\tilde{U}^{(s)}\right|^{4} \tilde{U}^{(s)}(\lambda, \cdot)
\end{array}\right),\left(e^{i x \xi}+e^{-i x \xi}\right) \underline{e}\right\rangle d \lambda \\
& \cdot \int_{0}^{s / 2} \frac{1}{s-\lambda^{\prime}} e^{+i(s-\lambda)\left(\xi^{\prime 2}+1\right)}\left\langle\left(\begin{array}{c}
x\left|\tilde{U}^{(s)}\right|^{4} \tilde{U}^{(s)}(\lambda, \cdot) \\
-x\left|\tilde{U}^{(s)}\right|^{4} \tilde{U}^{(s)}(\lambda, \cdot)
\end{array}\right),\left(e^{i x \xi^{\prime}}+e^{-i x \xi^{\prime}}\right) \sigma_{1} \underline{e}\right) d \lambda^{\prime} d s d t d \xi d \xi^{\prime} \mid \\
&
\end{aligned}
$$

as desired. 
The case $\max \left\{\lambda, \lambda^{\prime}\right\}>s / 2$ is more of the same. This concludes the proof of the estimate for the bilinear symplectic form. We have now also filled the gap in retrieving control over $\|C U\|_{L_{x}^{2}}$ : while the $\phi$ in the expression $\left\langle\tilde{U}^{2}-\overline{\tilde{U}}^{2}, \phi\right\rangle$ encountered there was time dependent, one easily checks that up to an error which leads to an absolutely integrable expression, one may replace this by a constant function.

This then also concludes the proof of Proposition 5.10 by the comments preceding case (B).

\subsection{Establishing control over the modulation parameters}

We formulate

Proposition 5.13. Let $\Gamma_{T}^{\prime}=T_{A, \lambda, \beta, \omega, \gamma, \mu} \Gamma_{T}, \Gamma_{T} \in A_{[0, T)}^{(10)}$. Then the iterative step (viii) (Section 4) is well-defined. Moreover,

$$
\begin{aligned}
& \sup _{0 \leq s<T}\langle s\rangle^{1 / 2-\delta_{1}}\left|v_{1}^{\prime}(s)\right|+\sum_{1 \leq k \leq[N / 3]}\left\|\langle s\rangle^{3 / 2-2 \delta_{1}} \frac{d^{k}}{d s^{k}} v_{1}^{\prime}(s)\right\|_{L^{M}[0, T]} \\
& +\sup _{0 \leq s<T}\langle s\rangle^{3 / 2-\delta_{1}}\left|\beta_{1}^{\prime}(s)\right|+\sum_{1 \leq k \leq[N / 3]}\left\|\langle s\rangle^{2-2 \delta_{1}} \frac{d^{k}}{d s^{k}} \beta_{1}^{\prime}(s)\right\|_{L^{M}[0, T]}\left\|\langle s\rangle^{3 / 2-2 \delta_{1}} \frac{d^{k}}{d s^{k}} \omega_{1}^{\prime}(s)\right\|_{L^{M}[0, T]} \\
& +\sup _{0 \leq s<T}\langle s\rangle^{3 / 2-\delta_{1}}\left|\omega_{1}^{\prime}(s)\right|+\sum_{1 \leq k \leq[N / 3]} \|\left\langle\sum_{1 \leq k \leq[N / 3]}\left\|\langle s\rangle^{3 / 2-2 \delta_{1}} \frac{d^{k}}{d s^{k}} \gamma_{1}^{\prime}(s)\right\|_{L^{M}[0, T]}\left\|\langle s\rangle^{5 / 2-2 \delta_{1}} \frac{d^{k}}{d s^{k}} \mu_{1}^{\prime}(s)\right\|_{L^{M}[0, T]} \lesssim \delta^{2} .\right. \\
& +\sup _{0 \leq s<T}\langle s\rangle^{1 / 2-\delta_{1}}\left|\gamma_{1}^{\prime}(s)\right|+\sum_{1 \leq k \leq[N / 3]} \|\langle s<T
\end{aligned}
$$

Proof. We commence by estimating $\beta_{1}^{\prime}=\beta^{\prime} v^{\prime}-b_{\infty}^{\prime}\left(\lambda_{\infty}^{\prime}\right)^{-1}$, which is given by the right hand side of $(3.8)$. Observe that schematically we have

$$
E_{2}(\sigma)=-\left\langle N, \tilde{\xi}_{2}\right\rangle+\sum_{a=0,1}(v-1)^{a} \lambda_{6}(\sigma)+(v-1)(\sigma)\left\langle\left(\begin{array}{c}
\tilde{U} \\
\overline{\tilde{U}}
\end{array}\right)_{\mathrm{dis}}, \phi\right\rangle .
$$

Thus we recover the first desired estimate for $\beta_{1}(s),\left|\beta_{1}(s)\right| \lesssim \delta^{2}\langle s\rangle^{-3 / 2+\delta_{1}}$, upon using Proposition 5.10 and its proof, if we also show that

$$
\left|\lambda_{\infty}^{-1}(s) \int_{s}^{T} \lambda_{\infty}(\sigma)(v-1)(\sigma)\left\langle\left(\begin{array}{c}
\tilde{U} \\
\tilde{\tilde{U}}
\end{array}\right)_{\mathrm{dis}}, \phi\right\rangle d \sigma\right| \lesssim\langle s\rangle^{-3 / 2+\delta_{1}} .
$$

We state 
Lemma 5.14. Let $\Gamma \in A_{[0, T)}^{(9)}$. Then

$$
\left|\int_{s}^{T} \lambda_{\infty}(\sigma)(v-1)(\sigma)\left\langle\left(\begin{array}{c}
\tilde{U} \\
\tilde{\tilde{U}}
\end{array}\right)_{\operatorname{dis}}(\sigma), \phi\right\rangle d \sigma\right| \lesssim\langle s\rangle^{-1 / 2+\delta_{1}}
$$

Proof. This is proved as usual by integration by parts in $\sigma$, and Duhamel-expanding $\left(\begin{array}{c}\tilde{U} \\ \tilde{U}\end{array}\right)_{\text {dis }}(\sigma)$. Thus we rewrite the expression as the sum of the terms

$$
\begin{aligned}
& \int_{-\infty}^{\infty} \int_{s}^{T} t(v-1)(t) \int_{0}^{t} e^{i(t-\lambda)\left(\xi^{2}+1\right)} \\
& \cdot \mathcal{F}\left(\begin{array}{c}
\left(1-e^{2 i\left(\Psi-\Psi_{\infty}\right)_{1}(t)-2 i\left(\Psi-\Psi_{\infty}\right)_{1}(\lambda)}\right) \overline{\tilde{U}^{(t)}(\lambda, \cdot)} \phi_{0}^{4} \\
\left(-1+e^{2 i\left(\Psi-\Psi_{\infty}\right)_{1}(t)-2 i\left(\Psi-\Psi_{\infty}\right)_{1}(\lambda)}\right) \tilde{U}^{(t)}(\lambda, \cdot) \phi_{0}^{4}
\end{array}\right)(\xi) \overline{\tilde{\mathcal{F}} \phi(\xi)} d \lambda d t d \xi, \\
& \int_{-\infty}^{\infty} \int_{s}^{T} t(v-1)(t) \int_{0}^{t} e^{i(t-\lambda)\left(\xi^{2}+1\right)} \mathcal{F}\left(\begin{array}{c}
\left|\tilde{U}^{(t)}\right|^{4}(\lambda, \cdot) \tilde{U}^{(t)}(\lambda, \cdot) \\
-\left|\tilde{U}^{(t)}\right|^{4}(\lambda, \cdot) \tilde{U}^{(t)}(\lambda, \cdot)
\end{array}\right)(\xi) \overline{\tilde{\mathcal{F}} \phi(\xi)} d \lambda d t d \xi,
\end{aligned}
$$

as well as faster decaying local terms which can be handled similarly to the first term. Let us look at the second term here, the first being treated as in the proof of Lemma 5.11 by integrations by parts and further Duhamel expansion. Perform an integration by parts, replacing this by the sum of suitable multiples of

$$
\begin{aligned}
& A=\int_{-\infty}^{\infty} s(v-1)(s) \int_{0}^{s} e^{i(s-\lambda)\left(\xi^{2}+1\right)} \mathcal{F}\left(\begin{array}{c}
\left|\tilde{U}^{(s)}\right|^{4}(\lambda, \cdot) \tilde{U}^{(s)}(\lambda, \cdot) \\
-\left|\tilde{U}^{(s)}\right|^{4}(\lambda, \cdot) \tilde{U}^{(s)}(\lambda, \cdot)
\end{array}\right)(\xi) \frac{\overline{\mathcal{F} \phi(\xi)}}{\xi^{2}+1} d \lambda d t d \xi, \\
& B=\int_{-\infty}^{\infty} \int_{s}^{T} \frac{d}{d t}[t(v-1)(t)]
\end{aligned}
$$

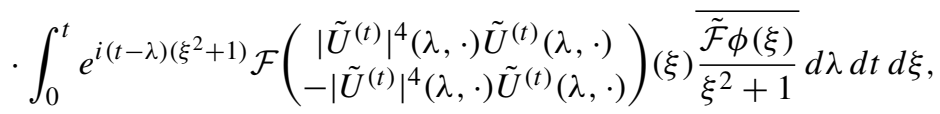

$$
\begin{aligned}
& C=\int_{-\infty}^{\infty} \int_{s}^{T} t(v-1)(t) \frac{d}{d t}\left[\lambda_{\infty}\left(\mu-\mu_{\infty}\right)(t)\right] \\
& \cdot \int_{0}^{t} e^{i(t-\lambda)\left(\xi^{2}+1\right)} \mathcal{F}\left(\begin{array}{c}
\left|\tilde{U}^{(t)}\right|^{4}(\lambda, \cdot) \partial_{x} \tilde{U}^{(t)}(\lambda, \cdot) \\
-\left|\tilde{U}^{(t)}\right|^{4}(\lambda, \cdot) \partial_{x} \tilde{U}^{(t)}(\lambda, \cdot)
\end{array}\right)(\xi) \frac{\overline{\mathcal{F} \phi(\xi)}}{\xi^{2}+1} d \lambda d t d \xi \\
& D=\int_{-\infty}^{\infty} \int_{s}^{T} t(v-1)(t) \frac{d}{d t}\left[\lambda_{\infty}\left(\mu-\mu_{\infty}\right)(t)\right]
\end{aligned}
$$

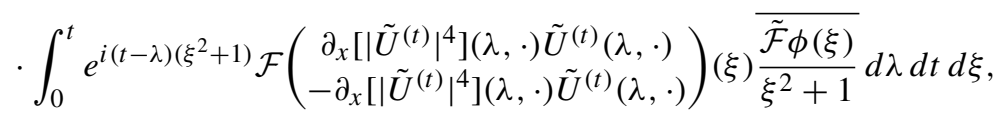

$$
\begin{aligned}
& E=\int_{-\infty}^{\infty} \int_{s}^{T} t(v-1)(t) \frac{d}{d t}\left[\Psi-\Psi_{\infty}\right]_{1}(t) \\
& \cdot \int_{0}^{t} e^{i(t-\lambda)\left(\xi^{2}+1\right)} \mathcal{F}\left(\begin{array}{l}
\left|\tilde{U}^{(t)}\right|^{4}(\lambda, \cdot) \tilde{U}^{(t)}(\lambda, \cdot) \\
\left|\tilde{U}^{(t)}\right|^{4}(\lambda, \cdot) \tilde{U}^{(t)}(\lambda, \cdot)
\end{array}\right)(\xi) \frac{\overline{\mathcal{F} \phi(\xi)}}{\xi^{2}+1} d \lambda d t d \xi, \\
& F=\int_{-\infty}^{\infty} \int_{s}^{T} t(v-1)(t) \mathcal{F}\left(\begin{array}{c}
|\tilde{U}|^{4}(t, \cdot) \tilde{U}(t, \cdot) \\
-|\tilde{U}|^{4}(t, \cdot) \tilde{U}(t, \cdot)
\end{array}\right)(\xi) \frac{\overline{\mathcal{F}} \phi(\xi)}{\xi^{2}+1} d t d \xi
\end{aligned}
$$


Now, for $A$, repeat the proof of $\mathrm{SLDE}^{68}$ to bound it by $\lesssim s^{1 / 2+\delta_{1}} s^{-3 / 2+\delta_{3}}$, better than what is needed. For $B$, use $\left|\frac{d}{d t}[t(v-1)(t)]\right| \lesssim t^{-1 / 2+2 \delta_{1}}$ (see [3.14)). $C$ is handled analogously. For $D$, observe that 69

$$
\begin{aligned}
\int_{-\infty}^{\infty} \int_{0}^{t} e^{i(t-\lambda)\left(\xi^{2}+1\right)} \mathcal{F}\left(\begin{array}{c}
\partial_{x}\left[\left|\tilde{U}^{(t)}\right|^{4}\right](\lambda, \cdot) \tilde{U}^{(t)}(\lambda, \cdot) \\
-\partial_{x}\left[\left|\tilde{U}^{(t)}\right|^{4}\right](\lambda, \cdot) \cdot \tilde{U}^{(t)}(\lambda, \cdot)
\end{array}\right)(\xi) \frac{\overline{\mathcal{F}} \phi(\xi)}{\xi^{2}+1} d \lambda d \xi \\
=\left\langle\int_{0}^{t} e^{i(t-\lambda) \mathcal{H}}\left(\begin{array}{c}
\partial_{x}\left[\left|\tilde{U}^{(t)}\right|^{4}\right](\lambda, \cdot) \tilde{U}^{(t)}(\lambda, \cdot) \\
-\partial_{x}\left[\left|\tilde{U}^{(t)}\right|^{4}\right](\lambda, \cdot) \tilde{U}^{(t)}(\lambda, \cdot)
\end{array}\right)_{\operatorname{dis}} d \lambda, \mathcal{H}^{-1} \phi\right\rangle .
\end{aligned}
$$

Proceeding as in the proof of SLDE, i.e., using (5.4) as well as pseudo-conformal almost conservation, we can bound the preceding expression by $\lesssim\langle T\rangle^{-3 / 2+\delta_{3}}$. Now one proceeds as for $C$ etc. Expression $E$ is like the one we started out with, but with an extra $\frac{d}{d t}\left[\Psi-\Psi_{\infty}\right]_{1}(t) \sim(v-1)(t)$. Finally, $F$ is more elementary, as we have

$$
\begin{aligned}
\left|\int_{-\infty}^{\infty} \mathcal{F}\left(\begin{array}{c}
|\tilde{U}|^{4}(t, \cdot) \tilde{U}(t, \cdot) \\
-|\tilde{U}|^{4}(t, \cdot) \tilde{U}(t, \cdot)
\end{array}\right)(\xi) \frac{\overline{\mathcal{F}} \phi(\xi)}{\xi^{2}+1} d \xi\right| & =\left|\left\langle\left(\begin{array}{c}
|\tilde{U}|^{4}(t, \cdot) \tilde{U}(t, \cdot) \\
-|\tilde{U}|^{4}(t, \cdot) \tilde{U}(t, \cdot)
\end{array}\right)_{\mathrm{dis}}, \mathcal{H}^{-1} \phi\right\rangle\right| \\
& \lesssim t^{-9 / 2+\epsilon\left(\delta_{2}\right)} .
\end{aligned}
$$

The derivatives of $\beta_{1}^{\prime}(s)$ are then estimated upon differentiating 3.8.

We proceed to $v_{1}^{\prime}$, which is defined via the right hand side of (3.7). Using identical reasoning as for $\beta_{1}^{\prime}$, one obtains the bound $\left|v_{1}^{\prime}(s)\right| \lesssim\langle s\rangle^{-1 / 2+\delta_{1}} \delta^{2}$. We can now define $a_{\infty}^{\prime}, b_{\infty}^{\prime}$ as in the iterative step in Section 4, which then also defines $\beta^{\prime}$. Further, we have, following step (viii) in Section 4,

$$
e^{-\int_{0}^{s}\left[\beta^{\prime} \nu^{\prime 2}+\frac{1}{4 i \kappa_{2}} E_{5}\right](\sigma) d \sigma}-c^{\prime} \lambda^{\prime}(s)^{-1} \lesssim \delta^{2}\langle s\rangle^{-3 / 2+\delta_{1}}
$$

Then define $c_{\infty}^{\prime}$ and $\omega_{1}^{\prime}(s)$ as in step (viii) of Section 4 . The bound $\left|\langle s\rangle^{3 / 2-\delta_{1}}\right| \omega_{1}^{\prime}(s) \mid \lesssim \delta^{2}$ is an immediate consequence of the preceding estimates as are the desired bounds for the derivatives of $\omega_{1}^{\prime}(s)$.

We next define $\mu^{\prime}(s)$ as in 4.1. Again using the definitions and the fine structure of $E_{4}$ etc. as well as the preceding estimates, we infer the existence of constants $v_{\infty}^{\prime}, y_{\infty}^{\prime}$ such that

$$
\mu^{\prime}(s)=\frac{2 v_{\infty}^{\prime} s+y_{\infty}^{\prime}}{a_{\infty}^{\prime}+b_{\infty}^{\prime} s}+o\left(\frac{1}{s}\right) .
$$

Also, observe that due to our definition of $\mu^{\prime}(s)$, we automatically have the consistency relation

Further, defining

$$
c_{\infty}^{\prime}=v_{\infty}^{\prime} a_{\infty}^{\prime}-\frac{b_{\infty}^{\prime} y_{\infty}^{\prime}}{2}
$$

$$
\mu_{1}^{\prime}(s)=\mu^{\prime}(s)-\frac{2 v_{\infty}^{\prime} s+y_{\infty}^{\prime}}{a_{\infty}^{\prime}+b_{\infty}^{\prime} s}
$$

68 SLDE refers to strong local dispersive estimate, i.e. Lemma 5.7

69 Of course, one should include the \pm subscripts for $\mathcal{F}, \tilde{\mathcal{F}}$. 
the desired bounds follow from the fine structure of $E_{4}$ etc. as well as the preceding estimates. Finally, defining $\gamma^{\prime}(s), \gamma_{1}^{\prime}(s)$ as in step (viii) of Section 4 , the desired bounds follow analogously.

Combining Lemma 5.4, Lemma5.7, Lemma 5.8, Lemma 5.9, and Proposition 5.13. we have finally proved Theorem 5.1 Continuity of the operator $T_{A, \lambda, \beta, \omega, \gamma, \mu}$ is straightforward and can be achieved using crude bounds, as we only work on a finite time interval. This then implies Theorem 4.5

Acknowledgments. The authors would like to thank Jean Bourgain and Carlos Kenig for helpful discussions and their interest in our work. The first author would also like to express his gratitude to the California Institute of Technology and the University of Chicago for generously hosting him during the Summer 2004 and Summer 2005, respectively.

The first author was partially supported by NSF grant DMS-0401177 and the second author by the NSF grant DMS-0300081 and a Sloan fellowship.

\section{References}

[Agm] Agmon, S.: Lectures on Exponential Decay of Solutions of Second-Order Elliptic Equations: Bounds on Eigenfunctions of $N$-body Schrödinger Operators. Math. Notes 29, Princeton Univ. Press, Princeton, NJ, University of Tokyo Press, Tokyo (1982) Zbl 0503.35001 MR 0745286

[BerCaz] Berestycki, H., Cazenave, T.: Instabilité des états stationnaires dans les équations de Schrödinger et de Klein-Gordon non linéaires. C. R. Acad. Sci. Paris Sér. I Math. 293, 489-492 (1981) Zbl 0492.35010 MR 0646873

[B] Bourgain, J.: Global Solutions of Nonlinear Schrödinger Equations. Amer. Math. Soc. Colloq. Publ. 46, Amer. Math. Soc. (1999) Zbl 0933.35178 MR 1691575

[BW] Bourgain, J., Wang, W.: Construction of blowup solutions for the nonlinear Schrödinger equation with critical nonlinearity. Ann. Scuola Norm. Sup. Pisa (4) 25, 197-215 (1997) Zbl 1043.35137 MR 1655515

[BusPer] Buslaev, V. S., Perelman, G. S.: Scattering for the nonlinear Schrödinger equation: states that are close to a soliton. Algebra i Analiz 4, no. 6, 63-102 (1992) (in Russian); English transl.: St. Petersburg Math. J. 4, 1111-1142 (1993) Zbl 0853.35112 MR 1199635

[CazLio] Cazenave, T., Lions, P.-L.: Orbital stability of standing waves for some nonlinear Schrödinger equations. Comm. Math. Phys. 85, 549-561 (1982) Zbl 0513.35007 MR 0677997

[ChriKis] Christ, M., Kiselev, A.: Maximal functions associated with filtrations. J. Funct. Anal. 179, 409-425 (2001) Zbl 0974.47025 MR 1809116

[Cuc] Cuccagna, S.: Stabilization of solutions to nonlinear Schrödinger equations. Comm. Pure Appl. Math. 54, no. 9, 1110-1145 (2001) Zbl 1031.35129 MR 2094268

[Flu] Flügge, S.: Practical Quantum Mechanics. Reprinting in one volume of Vols. I, II. Springer, New York (1974) Zbl 0934.81001 MR 0366248

[Froe] Fröhlich, J., Gustafson, S., Jonsson, B. L. G., Sigal, I. M.: Solitary wave dynamics in an external potential. Comm. Math. Phys. 250, 613-642 (2004) Zbl 1075.35075 MR 2094474 
[GolSch] Goldberg, M., Schlag, W.: Dispersive estimates for Schrödinger operators in dimensions one and three. Comm. Math. Phys. 251, 157-178 (2004) Zbl 1086.81077 MR 2096737

[Gri] Grillakis, M.: Analysis of the linearization around a critical point of an infinite dimensional Hamiltonian system. Comm. Pure Appl. Math. 43, 299-333 (1990) Zbl 0731.35010 MR 1040143

[Har] Hartman, P.: Ordinary Differential Equations. Corrected reprint of the second (1982) edition. Classics in Appl. Math. 38, SIAM, Philadelphia, PA (2002) Zbl 1009.34001 MR 1929104

[Kat] Kato, T.: Wave operators and similarity for some non-selfadjoint operators. Math. Ann. 162, 258-279 (1965/1966) Zbl 0139.31203 MR 0190801

[KriSch1] Krieger, J., Schlag, W.: Stable manifolds for all monic supercritical focusing nonlinear Schrödinger equations in one dimension. J. Amer. Math. Soc. 19, 815-920 (2006) Zbl pre05034395 MR 2219305

[KriSch2] Krieger, J., Schlag, W.: On the focusing critical semi-linear wave equation. Amer. J. Math. 129, 843-913 (2007) Zbl pre05170329 MR 2325106

[LiWig] Li, C., Wiggins, S.: Invariant Manifolds and Fibrations for Perturbed Nonlinear Schrödinger Equations. Appl. Math. Sci. 128, Springer, New York (1997) Zbl 0897.35070 MR 1475929

[MeRa] Merle, F., Raphael, P.: On universality of blow-up profile for $L^{2}$ critical nonlinear Schrödinger equation. Invent. Math. 156, 565-672 (2004) Zbl 1067.35110 MR 2061329

[Mur] Murata, M.: Asymptotic expansions in time for solutions of Schrödinger-type equations. J. Funct. Anal. 49, 10-56 (1982) Zbl 0499.35019 MR 0680855

[Per] Perelman, G.: On the formation of singularities in solutions of the critical nonlinear Schrödinger equation. Ann. Henri Poincaré 2, 605-673 (2001) Zbl 1007.35087 MR 1852922

[Ra] Raphael, P.: Stability of the $\log -\log$ bound for blow up solutions to the critical nonlinear Schrödinger equation. Math. Ann. 331, 577-609 (2005) Zbl 1082.35143 MR 2122541

[ReeSim4] Reed, M., Simon, B.: Methods of Modern Mathematical Physics. IV. Academic Press, New York (1979) Zbl 0401.47001 MR 0493421

[RodSch] Rodnianski, I., Schlag, W.: Time decay for solutions of Schrödinger equations with rough and time-dependent potentials. Invent. Math. 155, 451-513 (2004) Zbl 1063.35035 MR 2038194

[Sch1] Schlag, W.: Stable manifolds for an orbitally unstable NLS. Preprint (2004)

[Sch2] Schlag, W.: Dispersive estimates for Schrödinger operators: A survey. In: Mathematical Aspects of Nonlinear Dispersive Equations, Ann. of Math. Stud. 163, Princeton Univ. Press, 255-285 (2007) MR 2333215

[SogSmi] Smith, H., Sogge, C.: Global Strichartz estimates for nontrapping perturbations of the Laplacian. Comm. Partial Differential Equations 25, 2171-2183 (2000) Zbl 0972.35014 MR 1789924

[SofWei1] Soffer, A., Weinstein, M.: Multichannel nonlinear scattering for nonintegrable equations. Comm. Math. Phys. 133, 119-146 (1990) Zbl 0721.35082 MR 1071238

[SofWei2] Soffer, A., Weinstein, M.: Multichannel nonlinear scattering, II. The case of anisotropic potentials and data. J. Differential Equations 98, 376-390 (1992) Zbl 0795.35073 MR 1170476

[St] Stein, E.: Harmonic Analysis: Real-Variable Methods, Orthogonality, and Oscillatory Integrals. Princeton Univ. Press, Princeton, NJ (1993) Zbl 0821.42001 MR 1232192 
[TsaYau] Tsai, T.-P., Yau, H.-T.: Stable directions for excited states of nonlinear Schrödinger equations. Comm. Partial Differential Equations 27, 2363-2402 (2002) Zbl 1021.35113 MR 1944033

[Wei1] Weinstein, M. I.: Modulational stability of ground states of nonlinear Schrödinger equations. SIAM J. Math. Anal. 16, 472-491 (1985) Zbl 0583.35028 MR 0783974

[Wei2] Weinstein, M. I.: Lyapunov stability of ground states of nonlinear dispersive evolution equations. Comm. Pure Appl. Math. 39, 51-67 (1986) Zbl 0594.35005 MR 0820338 